$$
\begin{gathered}
\text { Universidade de São Paulo } \\
\text { Instituto de Física de São Carlos }
\end{gathered}
$$

DANILO MENDES DIAS DELFINO DA SILVA

\title{
Método da diagonalização na base de Krylov com agrupamento de linhas no ajuste de sinais ruidosos de espectroscopia por $R M$
}





\section{Método da diagonalização na base de Krylov com agrupamento de linhas no ajuste de sinais ruidosos de espectroscopia por RM}

Tese apresentada ao Programa de Pós-Graduação em Física do Instituto de Física de São Carlos da Universidade de São Paulo, para obtenção do título de Doutor em Ciências.

Área de Concentração: Física Aplicada Opção: Física Computacional

Orientador: Prof. Dr. Fernando Fernandes Paiva

Versão Corrigida

(Versão original disponível na Unidade que aloja o Programa) 
AUTORIZO A REPRODUÇÃO E DIVULGAÇÃO TOTAL OU PARCIAL DESTE TRABALHO, POR QUALQÜER MEIO CONVENCIONAL OU ELETRÔNICO PARA FINS DE ESTUDO E PESQUISA, DESDE QUE CITADA A FONTE.

da Silva, Danilo Mendes Dias Delfino

Método da diagonalização na base de Krylov com

agrupamento de linhas no ajuste de sinais ruidosos de espectroscopia por RM / Danilo Mendes Dias Delfino da Silva; orientador Fernando Fernandes Paiva - versão corrigida -- São Carlos, 2019.

$$
147 \mathrm{p} \text {. }
$$

Tese (Doutorado - Programa de Pós-Graduação em Física Aplicada Computacional) -- Instituto de Física de São Carlos, Universidade de São Paulo, 2019.

1. LLC-KBDM. 2. Agrupamento de linhas. 3. Método da diagonalização na base de Krylov. 4. Problema da inversão harmônica. 5. Espectroscopia por RM. I. Fernandes Paiva, Fernando, orient. II. Título. 


\section{AGRADECIMENTOS}

Ao meu orientador, Prof. Dr. Fernando Fernandes Paiva, pela orientação, compreensão e confiança.

A minha mulher, Ana, por me mostrar como vencer as maiores batalhas e ao mesmo tempo ter força para me incentivar e apoiar. Foi tudo mais fácil com seu apoio. Te amo!

A minha filha, Malu, por ter ouvido tantas vezes que "agora não dá" durante o desenvolvimento deste trabalho.

A minha mãe, Patricia e as minhas irmãs, Danielle e Letícia. Que saudade de vocês e desse cantinho. Me desculpem pela ausência. Amo muito vocês!

Ao meu padrasto, Dorival.

Aos meus sogros, Dona Ligia e Sr. Março.

Aos meus cunhados, Kátia e Rogério e ao Benjamin.

Aos meus amigos da eterna República das Bermudas.

Aos meus amigos de Barretos, os Goonies.

Ao pessoal do CIERMag, em especial ao professor Alberto, Edson e Mateus pelo conhecimento compartilhado durante estes anos.

A ESSS pelo incentivo contínuo no desenvolvimento da minha carreira.

A todas as pessoas que desenvolveram os métodos e tecnologias utilizados como base deste trabalho.

Um salve aos meus mais chegados, Ganso, Pudim, Cabeçudo, Yole, Manguaço, Arroz, Hélvio, Felipe, Zé, Haroldo, Bob, Murilo, Brunão, Cesão, Preta, Mia e Olivia. 
Este trabalho é dedicado à Dona Cida 


\section{RESUMO}

SILVA, D. M. D. D. Método da diagonalização na base de Krylov com agrupamento de linhas no ajuste de sinais ruidosos de espectroscopia por RM. 2019. 147 p. Tese (Doutorado em Ciências) - Instituto de Física de São Carlos, Universidade de São Paulo, São Carlos, 2019.

A Espectroscopia por Ressonância Magnética (ERM) in vivo é uma técnica não invasiva e não ionizante que permite a quantificação da concentração dos metabólitos presentes no corpo humano. No domínio da frequência, as ressonâncias são representadas por linhas espectrais ou picos obtidos através da Transformada de Fourier do sinal adquirido no domínio do tempo. A concentração de cada metabólito é proporcional ao número de núcleos visíveis à técnica de RM e à intensidade de seus respectivos picos. O sinal de um mesmo metabólito pode ser composto por vários picos no domínio da frequência, que podem estar sobrepostos aos demais em função das suas larguras e da resolução espectral. Métodos que realizam ajuste do espectro através da combinação linear de bases espectrais in vitro ou simuladas; e métodos que representam o sinal através de funções matemáticas estão disponíveis na literatura. Pertencente a esta última família, o Krylov Basis Diagonalization Method (KBDM), que representa o sinal através da sobreposição de senoides exponencialmente amortecidas no domínio do tempo foi pouco estudado na literatura no contexto da sua aplicação em ERM in vivo. Portanto, seu posicionamento em relação aos demais métodos da mesma família não é claro. Entre estes, o HLSVD-PRO (HLSVD with partial reorthogonalization), baseado no formalismo de espaço de estados, representa o estado da arte na literatura em tarefas como a remoção do sinal residual da água. Além de realizar um estudo sistemático sobre o KBDM, este trabalho propõe o desenvolvimento de um novo método denominado LLC-KBDM (Line List Clustering Krylov Basis Diagonalization Method), que realiza a promediação das linhas espectrais obtidas através das soluções do KBDM em múltiplos truncamentos do mesmo sinal no espaço de parâmetros. O agrupamento automático dos picos é realizado através do uso de técnicas de aprendizado de máquina não supervisionado. Comparações utilizando sinais de 900 espectros simulados em 9 níveis de ruído foram realizadas entre os métodos LLC-KBDM, KBDM e HLSVD-PRO. 0 desvio padrão do resíduo medido a partir da diferença entre o sinal simulado sem ruído e o sinal estimado, bem como o número de picos corretamente estimados são utilizados como métricas de comparação. Os resultados mostram que o KBDM é ligeiramente superior ao HLSVD-PRO em todos os níveis de ruído considerados. Por outro lado, o LLC-KBDM se mostrou mais 
adequado para sinais os mais ruidosos, embora algumas instabilidades numéricas tenham sido observadas no caso contendo o maior nível de ruído. Para sinais in vivo, a tarefa da remoção do sinal residual da água é comparada entre os mesmos métodos. Um teste estatístico que utiliza a curtose e a obliquidade das distribuições para verificação da normalidade dos valores do resíduo na região de supressão foi aplicado. De acordo com o teste para o nível de significância $\alpha=0.05$, o sinal residual da água para 10 dos 14 espectros foram adequadamente suprimidos pelo LLC-KBDM; enquanto o KBDM e HLSVD-PRO conseguiram o mesmo feito para apenas 6 e 7 espectros, respectivamente. Uma implementação para o LLC-KBDM está disponível em: https://github.com/danilomendesdias/llckbdm.

Palavras-chave: LLC-KBDM. Agrupamento de linhas espectrais. Método da diagonalização na base de Krylov. Problema da inversão harmônica. Espectroscopia por RM. 


\section{ABSTRACT}

SILVA, D. M. D. D. Krylov basis diagonalization method with spectral line clustering for noisy MRS data fitting. 2019. 147 p. Tese (Doutorado em Ciências) - Instituto de Física de São Carlos, Universidade de São Paulo, São Carlos, 2019.

In vivo Magnetic Resonance Spectroscopy (MRS) is a noninvasive and non-ionizing technique that allows the quantification of human body metabolite concentrations. In the frequency domain, resonances are represented by spectral lines or peaks obtained through the Fourier Transform of the acquired signal in the time domain. The metabolite concentration is proportional to the number of nuclei that are MR-visible and the respective peak intensities. The signal of a single metabolite can be composed by multiple peaks in the frequency domain, that may be overlapped with other peaks due large line-widths and spectral resolution. Methods that are capable of fitting the spectrum through linear combination of simulated or in vitro acquired spectral basis; and methods that represent the signal through model functions are found in the literature. Belonging to this last family of methods, Krylov Basis Diagonalization Method (KBDM) makes the assumption that the signal can be modeled through the superposition of exponentially damped sinusoids in the time domain. In the context of in vivo MRS application, this method has been little studied in the literature. Among these methods, HLSVD-PRO (HLSVD with partial reorthogonalization), which is based on the space-state formalism, represents the state-of-art in the literature for tasks such as residual water removal. Therefore, its position in relation to the other methods of the same family is not clear. Beyond the systematic study of the KBDM, this work also proposes the development of a new technique, named LLC-KBDM (Line List Clustering Krylov Basis Diagonalization Method), that makes use of spectral line list averaging in the parameter space of KBDM solutions that are obtained through multiple signal truncations. The automatic peak clustering is performed through the use of non-supervised machine learning techniques. Comparisons between LLCKBDM, KBDM and HLSVD-PRO were performed using 900 simulated spectra with 9 levels of noise. The standard deviation of the residual computed through the difference between the simulated signal without noise and the estimated signal, as well the number of correctly estimated peaks were used as benchmark metrics. The results show that KBDM is slightly superior to HLSVD-PRO for all noise levels considered. On the other hand, LLC-KBDM has demonstrated to be suitable for noisy signals, although numerical instabilities have been observed in 
the higher level of noise that was considered. For in vivo signals, the task that constitutes in the removal of residual water signal was chosen to perform the benchmark between the same methods. A statistical test for normality that uses the kurtosis and skewness of the distribution in the suppressed region was used. According to the results at significance level of $\alpha=0.05$, the residual water signal was successfully removed from 10 out of 14 spectra by LLC-KBDM; while KBDM and HLSVD-PRO, respectively, only removed 6 and 7 water signal residuals from the same spectra set.. An implementation for LLC-KBDM is available at: https://github.com/danilomendesdias/llckbdm.

Keywords: LLC-KBDM. Spectral line list clustering. Krylov basis diagonalization method. Harmonic inversion problem. MR spectroscopy. 


\section{LISTA DE FIGURAS}

Figura 2.1 - Espectro obtido a partir da Transformada de Fourier Discreta do sinal descrito pela Tabela 2.1 que simula uma aquisição no cérebro em equipamento ressonância magnética de $3 T$. A escala vertical se dá em unidade arbitrária. O eixo horizontal representa o deslocamento químico de cada ressonância em relação à frequência do tetrametilsilano.

Figura 2.2 - As componentes reais, imaginárias e magnitudes (de cima para baixo) dos sinais nos domínios do tempo, $\tilde{C}$, à esquerda e da frequência, $\tilde{S}$, à direita. $\tilde{C}$ e $\tilde{S}$ possuem $N=2048$ pontos e o intervalo de amostragem é de $\tau=0.5 \mathrm{~ms}$, resultando na largura de banda de $2 \mathrm{kHz}$.

Figura 2.3 - Erro absoluto médio (Equação 2.141), em função do parâmetro $M$ para vários valores de $J>K$ ao utilizar o KBDM no sinal descrito pela Tabela 2.1. A existência de cada curva obedece à condição $M \geq J$, que é uma restrição do KBDM resolvido através da fatoração SVD. Todas as curvas possuem comportamento semelhante quando $J>K$, indicando baixa sensibilidade ao parâmetro $J$ se esta restrição for obedecida. $\mathrm{O}$ pequeno aumento sistemático e proporcional a $J$ do erro é causado pelo acúmulo das contribuições das componentes espúrias de baixa amplitude que não foram filtradas nesta análise.

Figura 2.4 - Erro absoluto médio (Equação 2.141) em função do parâmetro $M$ para $J=14,15,16$ e 17 ao utilizar o KBDM no sinal descrito pela Tabela 2.1. O erros relativos elevados para $J=14$ e $J=15$ são esperados, pois neste caso o número de componentes estimados é necessariamente menor do que o número de componentes contidos no sinal. O comportamento das curvas respectivas a $J=16$ e $J=17$ é semelhante, mostrando que, neste caso, a componente espúria adicional estimada possui contribuição resultante desprezível no sinal estimado. 
Figura 2.5 - Espectros estimados utilizando o KBDM com $M=300$ para o sinal descrito pela Tabela 2.1 para $J=10,14,16$ e 300 . Para um sinal ideal modelado exatamente pela Equação 2.1, a implementação do KBDM é sensível ao parâmetro $J$ apenas quando $J<M$. . . . . . . . . . . . . 59

Figura 2.6 - Parte real do espectro computado a partir do sinal gerado pelas Equações 2.142 e 2.143 para $K=9$ e $\Delta f=220 \mathrm{~Hz}$.

Figura 2.7 - Erro absoluto médio da reconstrução do sinal apresentado na Figura 2.6 para $K=9$ e $\Delta f=220 \mathrm{~Hz}$. Neste caso, $M=K=9$ é suficiente para a reconstrução do sinal com precisão limitada pelo épsilon de máquina.

Figura 2.8 - Parte real do espectro computado a partir do sinal gerado pelas Equações 2.142 e 2.143 para $K=9$ e $\Delta f=50 \mathrm{~Hz}$. A superposição dos picos é acentuada neste caso.

Figura 2.9 - Impacto da sobreposição dos picos no erro absoluto médio durante a reconstrução do sinal apresentado na Figura 2.8. Desta vez, o ajuste realizado através do KBDM atinge precisão numérica máxima apenas quando $M \approx 29$.

Figura 2.10 -Valor ótimo do parâmetro $M$ do KBDM para o sinal descrito pelas Equações 2.142 e 2.143 em função da separação entre picos consecutivos no domínio da frequência $(\Delta f)$ e do número de picos $K=3,5,7$ e 9 . $M_{\text {ótimo }}$ é calculado a partir do valor mínimo do parâmetro $M$ onde o EAM é inferior a $10^{-10}$. O eixo vertical é apresentado em escala logarítmica.

Figura 2.11 -Erros absolutos médios (EAM) na presença de ruído com níveis definidos pelos desvios padrões $\sigma=0.001,0.003,0.01$ e 0.03 para o sinal parametrizado pela Tabela 2.1. Para esta análise, foram realizadas 10 observações para cada valor do parâmetro $M \operatorname{com} J=\min (16, M)$ e $M=5,10,15, \ldots, 400$. As linhas escuras representam o valor médio obtido paras os valores observados do EAM e o sombreamento em torno das curvas simbolizam as margens de erro estimadas através dos respectivos desvios padrões. 
Figura 2.12 -Erro absoluto médio (EAM) em função do nível de ruído determinado pelo desvio padrão $\sigma$ da distribuição que o descreve para as estimativas calculadas através do KBDM com $M=400$ e $J=16$. Ambos os eixos são apresentados em escala logarítmica.

Figura 2.13 -Exemplos de espectros estimados a partir do KBDM para sinais ruidosos. Os níveis de ruído variam do menor para o maior (de cima para baixo) para os valores de desvio padrão $\sigma=0.001,0.003,0.01$ e 0.03 . Do lado direito são apresentados os sinais de entrada e do lado esquerdo as estimativas obtidas através do KBDM (curva preta) e os respectivos resíduos (curva cinza) calculados a partir da diferença entre o espectro obtido e o valor verdadeiro sem a presença de ruído. Em todas as estimativas foram utilizados os parâmetros $M=400$ e $J=16 . \quad$. . . 65

Figura 2.14 -Erro absoluto médio (EAM) das estimativas do KBDM no sinal descrito pela Tabela 2.1 em função do parâmetro $M$ para diferentes valores valores de $J$ na presença de ruído gaussiano com $\sigma=0.01$. Comparado aos valores obtidos para o sinal sem ruído na Figura 2.4, a diferença entre os casos $J \geq K$ e $J<K$ é muito menor para este nível de ruído.

Figura 2.15 -Valores singulares obtidos através da implementação SVD utilizada para a matriz $\tilde{U}^{0}$ em função dos níveis de ruído do sinal. . . . . . . . . . . . . . 66

Figura 3.1 - Demonstração do uso de promediação da amplitude $\left(a_{k}\right)$ de $W=80$ linhas estimadas pelo $\operatorname{KBDM}(M=300,302, \ldots, 458)$ em sinais ruidosos $(\sigma=0.004)$ para os picos $1,2,3,4$ e 6 da Tabela 2.1. As linhas foram agrupadas de maneira supervisionada através dos melhores valores obtidos para o critério de similaridade entre os componentes estimados e verdadeiros.

Figura 3.2 - Demonstração do uso de promediação da amplitude $\left(a_{k}\right)$ de $W=80$ linhas estimadas pelo $\operatorname{KBDM}(M=300,302, \ldots, 458)$ em sinais ruidosos $(\sigma=0.004)$ para os picos 7, 8, 9, 10 e 11 da Tabela 2.1. As linhas foram agrupadas de maneira supervisionada através dos melhores valores obtidos para o critério de similaridade entre os componentes estimados e verdadeiros. 
Figura 3.3 - Demonstração do uso de promediação da amplitude $\left(a_{k}\right)$ de $W=80$ linhas estimadas pelo $\operatorname{KBDM}(M=300,302, \ldots, 458)$ em sinais ruidosos $(\sigma=0.004)$ para os picos $12,13,14,15$ e 16 da Tabela 2.1. As linhas foram agrupadas de maneira supervisionada através dos melhores valores obtidos para o critério de similaridade entre os componentes estimados e verdadeiros.

Figura 3.4 - Demonstração do uso de promediação da frequência $\left(f_{k}\right)$ de $W=80$ linhas estimadas pelo $\operatorname{KBDM}(M=300,302, \ldots, 458)$ em sinais ruidosos $(\sigma=0.004)$ para os picos $1,2,3,4$ e 6 da Tabela 2.1. As linhas foram agrupadas de maneira supervisionada através dos melhores valores obtidos para o critério de similaridade entre os componentes estimados e verdadeiros.

Figura 3.5 - Demonstração do uso de promediação da frequência $\left(f_{k}\right)$ de $W=80$ linhas estimadas pelo $\operatorname{KBDM}(M=300,302, \ldots, 458)$ em sinais ruidosos $(\sigma=0.004)$ para os picos 7, 8, 9, 10 e 11 da Tabela 2.1. As linhas foram agrupadas de maneira supervisionada através dos melhores valores obtidos para o critério de similaridade entre os componentes estimados e verdadeiros.

Figura 3.6 - Demonstração do uso de promediação da frequência $\left(f_{k}\right)$ de $W=80$ linhas estimadas pelo $\operatorname{KBDM}(M=300,302, \ldots, 458)$ em sinais ruidosos $(\sigma=0.004)$ para os picos $12,13,14,15$ e 16 da Tabela 2.1. As linhas foram agrupadas de maneira supervisionada através dos melhores valores obtidos para o critério de similaridade entre os componentes estimados e verdadeiros.

Figura 3.7 - Demonstração do uso de promediação da taxa de relaxação $\left(\gamma_{k}\right)$ de $W=80$ linhas estimadas pelo $\operatorname{KBDM}(M=300,302, \ldots, 458) \mathrm{em}$ sinais ruidosos $(\sigma=0.004)$ para os picos $1,2,3,4$ e 6 da Tabela 2.1. As linhas foram agrupadas de maneira supervisionada através dos melhores valores obtidos para o critério de similaridade entre os componentes estimados e verdadeiros. 
Figura 3.8 - Demonstração do uso de promediação da taxa de relaxação $\left(\gamma_{k}\right)$ de $W=80$ linhas estimadas pelo $\operatorname{KBDM}(M=300,302, \ldots, 458) \mathrm{em}$ sinais ruidosos $(\sigma=0.004)$ para os picos 7, 8, 9, 10 e 11 da Tabela 2.1. As linhas foram agrupadas de maneira supervisionada através dos melhores valores obtidos para o critério de similaridade entre os componentes estimados e verdadeiros.

Figura 3.9 - Demonstração do uso de promediação da taxa de relaxação $\left(\gamma_{k}\right)$ de $W=80$ linhas estimadas pelo $\operatorname{KBDM}(M=300,302, \ldots, 458) \mathrm{em}$ sinais ruidosos $(\sigma=0.004)$ para os picos $12,13,14,15$ e 16 da Tabela 2.1. As linhas foram agrupadas de maneira supervisionada através dos melhores valores obtidos para o critério de similaridade entre os componentes estimados e verdadeiros.

Figura 3.10 -Exemplo do perfil das taxas de reconstruções bem sucedidas dos picos da Tabela 2.1 para o sinal da Figura 2.1 em função do nível de ruído. As barras cinzas verticais representam valores médios das taxas de estimativas bem sucedidas (Equação 3.5) para 30 realizações contendo cada nível de ruído $(\sigma=0.01,0.02,0.04,0.08)$. As barras vermelhas representam o desvio padrão. As amostras foram obtidas através de múltiplos truncamentos do sinal $(M=300,302, \ldots, 458$ e $W=80)$.

Figura 3.11 -Conjunto de dados bidimensional ilustrando os critérios de agrupamento do algoritmo DBSCAN. Para o agrupamento mostrado, $N_{\min }=4 \mathrm{e}$ $\epsilon_{\min }$ é representado pelos círculos ao redor de cada amostra. Os pontos vermelhos (incluindo o ponto $A$ ) pertencem ao núcleo do cluster; os pontos amarelos ( $B$ e $C$ ), embora não pertencentes ao núcleo, também fazem parte do cluster; e apenas o ponto $N$ (em azul) não faz parte do agrupamento.

Figura 3.12 -Função objetiva, $f_{o b j}\left(N_{\min }, \epsilon_{\min }\right)$, para otimização dos parâmetros $\epsilon_{\min }$ e $N_{\min }$ do algoritmo DBSCAN na busca por linhas espectrais médias através de um conjunto de estimativas do KBDM feitas em múltiplos truncamentos do sinal. As regiões de maior erro são as mais escuras, enquanto as mais claras representam menores diferenças residuais entre o sinal estimado e o sinal de entrada. O marcador $X$ em preto denota o ponto mínimo global dentro da região de busca $\left(\epsilon_{\min } \approx 0.0193 \mathrm{e}\right.$ $\left.N_{\text {min }}=6\right)$. 
Figura 3.13 -Demonstração do ajuste do sinal sintético descrito pela Tabela $2.1 \mathrm{com}$ adição de ruído gaussiano $(\sigma=0.02)$ através do agrupamento de linhas espectrais estimadas pelo KBDM utilizando o algoritmo DBSCAN. Os utilizados pelo DBSCAN $\left(\epsilon_{\min } \approx 0.0193\right.$ e $\left.N_{\min }=6\right)$ foram obtidos através do processo de otimização demonstrado na Figura 3.12. . . . . . 89

Figura 3.14 -Diagrama de dispersão para os tempos de relaxação transversal $\left(\gamma_{k}^{-1}\right)$ e as amplitudes $a_{k}$ das linhas estimadas para o sinal contendo ruído mostrado na Figura 3.13. Os pequenos círculos representam as múltiplas estimativas obtidas através do processo de amostragem do KBDM com $M=200,210,220, \ldots, 390$ e $J=30$. Os círculos pretos são os pontos filtrados pelo DBSCAN, enquanto os círculos coloridos representam as linhas de um mesmo cluster. Os marcadores $\mathbf{X}$ simbolizam os centroides de cada cluster, utilizados como estimativa final do método para cada linha espectral. Os marcadores + representam as linhas verdadeiras apresentadas na Tabela 2.1. Enquanto as estimativas genuínas tendem a formar aglomerados, as linhas espúrias possuem alta dispersão. Ao todo, 16 linhas foram estimadas. Uma delas, no entanto, de caráter espúrio. Em função do nível de ruído, o pico \#5 não foi detectado.

Figura 3.15 -Diagrama de dispersão para os tempos de relaxação transversal $\left(\gamma_{k}^{-1}\right)$ e as frequências $f_{k}$ das linhas espectrais estimadas. Os pequenos círculos representam as múltiplas estimativas obtidas através do processo de amostragem do KBDM com $M=200,210,220, \ldots, 390$ e $J=30$. Os círculos pretos são os pontos filtrados pelo DBSCAN, enquanto os círculos coloridos representam as linhas de um mesmo cluster. Os marcadores $\mathbf{X}$ simbolizam os centroides de cada cluster, utilizados como estimativa final do método para cada linha espectral. Os marcadores + representam as linhas verdadeiras apresentadas na Tabela 2.1. Ao todo, 16 linhas foram estimadas. Uma delas, no entanto, de caráter espúrio. Em função do nível de ruído, o pico \#5 não foi detectado. . . 93 
Figura 3.16 -Diagrama de dispersão para as amplitudes $a_{k}$ e as frequências $f_{k}$ das linhas espectrais estimadas. Os pequenos círculos representam as múltiplas estimativas obtidas através do processo de amostragem do KBDM com $M=200,210,220, \ldots, 390$ e $J=30$. Os círculos pretos são os pontos filtrados pelo DBSCAN, enquanto os círculos coloridos representam as linhas de um mesmo cluster. Os marcadores $\mathbf{X}$ simbolizam os centroides de cada cluster, utilizados como estimativa final do método para cada linha espectral. Os marcadores + representam as linhas verdadeiras apresentadas na Tabela 2.1. Ao todo, 16 linhas foram estimadas. Uma delas, no entanto, de caráter espúrio. Em função do nível de ruído, o pico \#5 não foi detectado. Fonte: elaborada pelo autor.

Figura 3.17 -Plano complexo para para os autovalores da equação 2.66.

Figura 3.18 -Componentes reais e imaginárias dos autovalores $\tilde{\mu}_{k}=e^{\widetilde{\Omega}_{k} i \tau}$ para os parâmetros do sinal simulado descrito pela Tabela 2.1. Os tamanhos dos marcadores são proporcionais às amplitudes de cada componente.

Figura 3.19 -Plano complexo para os autovalores transformados pela Equação 3.37. A separação radial é obtida em detrimento da separação de frequências para as componentes com menores valores de $r\left(\gamma_{k}\right)$. O pontos pretos são componentes amostradas pelo KBDM para um sinal simulado com ruído $(\sigma=0.03)$ a partir dos parâmetros da Tabela 2.1. Os marcadores $\mathbf{X}$ coloridos representam os valores verdadeiros dos parâmetros. . . . . 99

Figura 3.20 -Diagramas de dispersão para cada par de componentes do vetor $\mathbf{x}=$ $\left(x^{1}, x^{2}, x^{3}, x^{4}\right) \in \mathcal{X}$ após padronização dados (Equações 3.40-3.43 e 3.46). O pontos pretos são componentes amostradas pelo KBDM para um sinal simulado com ruído $(\sigma=0.03)$ a partir dos parâmetros da Tabela 2.1. Os marcadores $\mathbf{X}$ coloridos representam os valores verdadeiros dos parâmetros. 
Figura 3.21 -Fluxograma do algoritmo LLC-KBDM. O processo de amostragem utilizando o KBDM é seguido pela otimização do agrupamento de linhas. Após a escolha do melhor conjunto de parâmetros, as linhas filtradas são utilizadas na construção do sinal parcial. A partir da diferença entre o sinal parcial total estimado e o sinal de entrada, o teste de convergência é aplicado. Se a convergência for alcançada, o algoritmo é encerrado. Caso contrário, o sinal residual é processado novamente e as etapas anteriores se repetem até que o número máximo de iterações seja excedido ou a convergência seja obtida. . . . . . . . . . . . . . . . 105

Figura 4.1 - Demonstração do MRSuppressor na leitura de dados de ERM in vivo em formato comercial Philips (SDAT/SPAR). Após a escolha do diretório raiz, o usuário pode visualizar o dado no domínio do tempo ou da frequência. As componentes reais, imaginárias e também a magnitude do dado representado em notação complexa podem ser simultaneamente visualizadas.

Figura 4.2 - Visualização das estimativas do espectro e parâmetros das linhas processadas pelo KBDM no MRSuppressor para sinal ruidoso simulado através dos parâmetros da Tabela 2.1.

Figura 5.1 - Desvio padrão do sinal residual calculado a partir da diferença entre o sinal estimado e o sinal verdadeiro (gerado a partir da Tabela 2.1, sem adição de ruído) em função dos níveis de ruídos sintéticos caracterizados pelos desvios padrões $\sigma=0.01,0.015,0.024,0.025,0.03,0.035,0.04$, $0.045,0.05$.

Figura 5.2 - Número médio de linhas corretamente estimadas em função dos níveis de ruídos para cada método estudado.

Figura 5.3 - Acurácia das estimativas bem sucedidas de cada pico para o nível de ruído caracterizado por $\sigma=0.01$ em função do método utilizado. Por não ter sido detectado em nenhum dos casos, o pico \#5 foi omitido do gráfico.

Figura 5.4 - Acurácia das estimativas bem sucedidas de cada pico para o nível de ruído caracterizado por $\sigma=0.015$ em função do método utilizado. Por não ter sido detectado em nenhum dos casos, o pico \#5 foi omitido do gráfico. 
Figura 5.5 - Acurácia das estimativas bem sucedidas de cada pico para o nível de ruído caracterizado por $\sigma=0.02$ em função do método utilizado. Por não ter sido detectado em nenhum dos casos, o pico \#5 foi omitido do gráfico.

Figura 5.6 - Acurácia das estimativas bem sucedidas de cada pico para o nível de ruído caracterizado por $\sigma=0.025$ em função do método utilizado. Por não ter sido detectado em nenhum dos casos, o pico \#5 foi omitido do gráfico.

Figura 5.7 - Acurácia das estimativas bem sucedidas de cada pico para o nível de ruído caracterizado por $\sigma=0.03$ em função do método utilizado. Por não ter sido detectado em nenhum dos casos, o pico \#5 foi omitido do gráfico.

Figura 5.8 - Acurácia das estimativas bem sucedidas de cada pico para o nível de ruído caracterizado por $\sigma=0.035$ em função do método utilizado. Por não ter sido detectado em nenhum dos casos, o pico \#5 foi omitido do gráfico.

Figura 5.9 - Acurácia das estimativas bem sucedidas de cada pico para o nível de ruído caracterizado por $\sigma=0.04$ em função do método utilizado. Por não ter sido detectado em nenhum dos casos, o pico \#5 foi omitido do gráfico.

Figura 5.10 -Acurácia das estimativas bem sucedidas de cada pico para o nível de ruído caracterizado por $\sigma=0.045$ em função do método utilizado. Por não ter sido detectado em nenhum dos casos, o pico \#5 foi omitido do gráfico.

Figura 5.11 -Acurácia das estimativas bem sucedidas de cada pico para o nível de ruído caracterizado por $\sigma=0.025$ em função do método utilizado. Por não ter sido detectado em nenhum dos casos, o pico \#5 foi omitido do gráfico. 
Figura 5.12 -Resultados obtidos para o espectro \#1. As linhas verticais tracejadas em A) denotam a região onde a água foi suprimida. Os pontos pretos nos diagramas de caixa em D) indicam a presença de outliers, caracterizado pela presença de sinal remanescente na região após o procedimento. O sinal completo denota o espectro total, antes da remoção da água.

Figura 5.13 -Resultados obtidos para o espectro \#2. As linhas verticais tracejadas em A) denotam a região onde a água foi suprimida. Os pontos pretos nos diagramas de caixa em D) indicam a presença de outliers, caracterizado pela presença de sinal remanescente na região após o procedimento. O sinal completo denota o espectro total, antes da remoção da água.

Figura 5.14 -Resultados obtidos para o espectro \#3. As linhas verticais tracejadas em A) denotam a região onde a água foi suprimida. Os pontos pretos nos diagramas de caixa em D) indicam a presença de outliers, caracterizado pela presença de sinal remanescente na região após o procedimento. O sinal completo denota o espectro total, antes da remoção da água.

Figura 5.15 -Resultados obtidos para o espectro \#4. As linhas verticais tracejadas em A) denotam a região onde a água foi suprimida. Os pontos pretos nos diagramas de caixa em D) indicam a presença de outliers, caracterizado pela presença de sinal remanescente na região após o procedimento. O sinal completo denota o espectro total, antes da remoção da água.

Figura 5.16-Resultados obtidos para o espectro \#5. As linhas verticais tracejadas em A) denotam a região onde a água foi suprimida. Os pontos pretos nos diagramas de caixa em D) indicam a presença de outliers, caracterizado pela presença de sinal remanescente na região após o procedimento. O sinal completo denota o espectro total, antes da remoção da água. 
Figura 5.17 -Resultados obtidos para o espectro \#6. As linhas verticais tracejadas em A) denotam a região onde a água foi suprimida. Os pontos pretos nos diagramas de caixa em D) indicam a presença de outliers, caracterizado pela presença de sinal remanescente na região após o procedimento. O sinal completo denota o espectro total, antes da remoção da água.

Figura 5.18 -Resultados obtidos para o espectro \#7. As linhas verticais tracejadas em A) denotam a região onde a água foi suprimida. Os pontos pretos nos diagramas de caixa em D) indicam a presença de outliers, caracterizado pela presença de sinal remanescente na região após o procedimento. O sinal completo denota o espectro total, antes da remoção da água.

Figura 5.19 -Resultados obtidos para o espectro \#8. As linhas verticais tracejadas em A) denotam a região onde a água foi suprimida. Os pontos pretos nos diagramas de caixa em D) indicam a presença de outliers, caracterizado pela presença de sinal remanescente na região após o procedimento. O sinal completo denota o espectro total, antes da remoção da água.

Figura 5.20 -Resultados obtidos para o espectro \#9. As linhas verticais tracejadas em A) denotam a região onde a água foi suprimida. Os pontos pretos nos diagramas de caixa em D) indicam a presença de outliers, caracterizado pela presença de sinal remanescente na região após o procedimento. O sinal completo denota o espectro total, antes da remoção da água.

Figura 5.21 -Resultados obtidos para o espectro \#10. As linhas verticais tracejadas em A) denotam a região onde a água foi suprimida. Os pontos pretos nos diagramas de caixa em D) indicam a presença de outliers, caracterizado pela presença de sinal remanescente na região após o procedimento. O sinal completo denota o espectro total, antes da remoção da água. 
Figura 5.22 -Resultados obtidos para o espectro \#11. As linhas verticais tracejadas em A) denotam a região onde a água foi suprimida. Os pontos pretos nos diagramas de caixa em D) indicam a presença de outliers, caracterizado pela presença de sinal remanescente na região após o procedimento. O sinal completo denota o espectro total, antes da remoção da água.

Figura 5.23 -Resultados obtidos para o espectro \#12. As linhas verticais tracejadas em A) denotam a região onde a água foi suprimida. Os pontos pretos nos diagramas de caixa em D) indicam a presença de outliers, caracterizado pela presença de sinal remanescente na região após o procedimento. O sinal completo denota o espectro total, antes da remoção da água. . . . . . . . . . . . . . . . . . . . . . . . . . . . . . . . . . 134

Figura 5.24 -Resultados obtidos para o espectro \#13. As linhas verticais tracejadas em A) denotam a região onde a água foi suprimida. Os pontos pretos nos diagramas de caixa em D) indicam a presença de outliers, caracterizado pela presença de sinal remanescente na região após o procedimento. O sinal completo denota o espectro total, antes da remoção da água. . . . . . . . . . . . . . . . . . . . . . 135

Figura 5.25 -Resultados obtidos para o espectro \#14. As linhas verticais tracejadas em A) denotam a região onde a água foi suprimida. Os pontos pretos nos diagramas de caixa em D) indicam a presença de outliers, caracterizado pela presença de sinal remanescente na região após o procedimento. O sinal completo denota o espectro total, antes da remoção da água. 


\section{LISTA DE TABELAS}

Tabela 2.1 - Amplitude no domínio do tempo, taxa de relaxação transversal $\left(\gamma^{-1}\right)$, frequência em Hertz $(f)$ e deslocamento químico em ppm $(\delta)$ para cada componente ( $K=16$ ao total) do sinal gerado a partir do modelo dado pela Equação 2.1. As fases de todos os componentes são nulas $\left(\varphi_{k}=0\right) .54$

Tabela 3.1 - Parâmetros das linhas obtidas através de múltiplas amostragens do KBDM com agrupamento de linhas através do DBSCAN para o exemplo mostrado na Figura 3.13. . . . . . . . . . . . . . . . . . . . . . . . 90

Tabela 5.1 - Teste de hipóteses para a distribuição do resíduo na região onde o sinal da água residual foi suprimido para três níveis de significância ( $\alpha=$ $0.001,0.01,0.05)$. A estatística $k^{2}$ combina a curtose e a obliquidade da distribuição testada. . . . . . . . . . . . . . . . . . . 122

Tabela 5.2 - Quantidade de espectros onde a hipótese nula não foi rejeitada para cada método em função dos níveis significância $(\alpha)$. . . . . . . . . 122 



\section{LISTA DE ACRÔNIMOS}

API - Application User Interface

BIRCH - Balanced Iterative Reducing and Clustering using Hierarchies

DBSCAN - Density-based spatial clustering of applications with noise

EAM - Erro Absoluto Médio

ERM - Espectroscopia por Ressonância Magnética

FDM - Filter Diagonalization Method

FEM - Força Eletromotriz

FID - Free Induction Decay

HDBSCAN - Hierarchical Density-based spatial clustering of applications with noise

HLSVD - Hankel Lanczos Singular Value Decomposition

HLSVD-IRL - HLSVD with implicitly restarted Lanczos

HLSVD-PRO - HLSVD with partial reorthogonalization

HSVD - Hankel Singular Value Decomposition

HTLS - Hankel Total Least Squares

KBDM - Krylov Basis Diagonalization Method

LAPACK - Linear Algebra PACKage

LLC-KBDM - Line List Clustering Krylov Basis Diagonalization Method

LPSVD - Linear Prediction Singular Value Decomposition

MKL - Math Kernel Library

OPTICS - Ordering Points To Identify the Clustering Structure

PAG - Problema de Autovalor Generalizado

PIH - Problema da Inversão Harmônica

RM - Ressonância Magnética 
RF - Radiofrequência

RSR - Relação Sinal-Ruído

SVD - Singular Value Decomposition

TDD - Test Driven Development

TF - Transformada de Fourier

TRF - Transformada Rápida de Fourier 


\section{SUMÁRIO}

1 Introdução

2 Método da Diagonalização na Base de Krylov

$2.1 \quad$ Problema da Inversão Harmônica . . . . . . . . . . . . . . . . . . . . 33

2.2 A conexão entre o Problema da Inversão Harmônica e um sistema dinâmico fictício . . . . . . . . . . . . . . . . . . . . . 36

2.3 O KBDM como solução do HIP . . . . . . . . . . . . . . . . . . . 40

2.4 Solução do Problema de Autovalores Generalizado . . . . . . . . . . . . . . . 45

2.4.1 Exemplo analítico . . . . . . . . . . . . . . . . . . . 45

2.4 .2 Decomposição em valores singulares . . . . . . . . . . . . . . . . . . . . 48

2.4.3 Outros métodos para solução do PAG . . . . . . . . . . . . . . . . . . . . . 51

$2.5 \quad$ Implementação numérica . . . . . . . . . . . . . . . . . . . . 52

2.5 .1 Sinal simulado f . . . . . . . . . . . . . . . . . . . . . 52

2.5.2 Análise de sensibilidade dos parâmetros do KBDM . . . . . . . . . . . . . . 54

$3 \quad$ Promediação através do agrupamento de linhas espectrais $\quad 67$

$3.1 \quad$ Introdução . . . . . . . . . . . . . . . . . . . . . . 67

$3.2 \quad$ Amostragem . . . . . . . . . . . . . . . . . . 68

3.2.1 Adição de pseudo-ruído . . . . . . . . . . . . . . . . . 68

3.2.2 Múltiplos truncamentos . . . . . . . . . . . . . . . . . . . . . . 69

3.2.3 Aspectos da amostragem nas diferentes abordagens . . . . . . . . . . . . . . 69

3.2.3.1 Promediação no domínio temporal ou espectral . . . . . . . . . . . . . . . . 70

3.2.3.2 Promediação no espaço de parâmetros . . . . . . . . . . . . . . . . . . 70

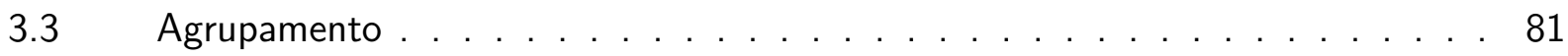


3.3.1 Algoritmos de agrupamento de dados . . . . . . . . . . . . . . . . . . . . 81

3.3.2 Agrupamento de linhas espectrais estimadas pelo KBDM . . . . . . . . . . . . 84

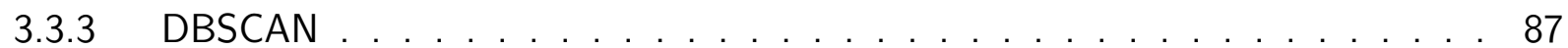

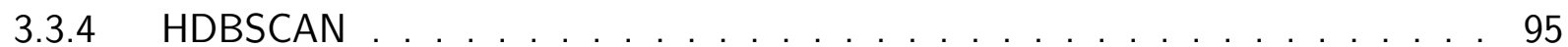

3.4 Transformações no espaço de dados . . . . . . . . . . . . . . . . . . . 96

$3.5 \quad$ Ajuste iterativo . . . . . . . . . . . . . . . . . . . . . . . . . . . . .

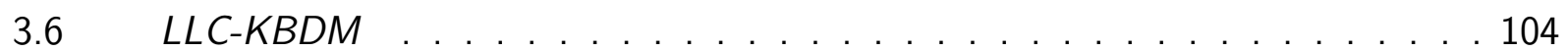

4 Desenvolvimento de ferramentas computacionais para o KBDM e LLC$\begin{array}{ll}\text { KBDM } & 107\end{array}$

$5 \quad$ Aplicações e Resultados $\quad 111$

5.1 Sinal simulado com adição de ruído . . . . . . . . . . . . . . . . . 111

5.1 .1 Resultados e discussões . . . . . . . . . . . . . . . . . . . . 112

5.2 Espectroscopia in vivo do cérebro humano: remoção do sinal da água residual . 119

5.2.1 Descrição do estudo . . . . . . . . . . . . . . . . . . . . . . . . . . 119

5.2 .2 Resultados e discussões . . . . . . . . . . . . . . . . . . . 121

$6 \quad$ Conclusão e trabalhos futuros 137

$\begin{array}{ll}\text { REFERÊNCIAS } & 139\end{array}$ 


\section{CAPÍtulo 1}

\section{Introdução}

A Espectroscopia por Ressonância Magnética (ERM) in vivo é uma técnica não invasiva e não ionizante que permite a quantificação da concentração dos metabólitos presentes no corpo humano e de outros seres vivos. Para mencionar alguns exemplos de aplicação, estudos sobre diversas patologias como a Doença de Alzheimer, contaminação pelo vírus HIV (Human Immunodeficiency Virus) e algumas doenças psiquiátricas, como a esquizofrenia ou o transtorno obsessivo compulsivo foram realizados através do uso da espectroscopia por ressonância magnética no cérebro. (1-5)

A Transformada Rápida de Fourier (TRF) (6) do FID (Free Induction Decay) permite a representação do sinal de ERM no domínio da frequência, também denominada espectro. $O$ FID é o sinal típico no domínio do tempo adquirido através do espectrômetro de Ressonância Magnética (RM). (7)

O spin é uma forma de momentum angular intrínseco das partículas quânticas, sem análogo clássico e está presente nas partículas que possuem carga e em alguns núcleos. (8-9) No caso da Ressonância Magnética Nuclear, o spin do núcleo atômico é a entidade física de interesse. O termo nuclear neste contexto, no entanto, tem sido omitido a partir dos últimos anos para não causar confusão com os assuntos relacionados à física nuclear.

A interação de um sistema de spins com um campo magnético estático de alta intensidade é capaz de produzir uma magnetização em escala macroscópica na mesma direção deste campo magnético, que caracteriza sua condição de equilíbrio nestas condições. Através da aplicação de uma sequência de pulsos de radiofrequência (RF) por meio de bobinas transmissoras, a magnetização pode ser retirada da condição de equilíbrio. Durante a aplicação destes pulsos, o campo magnético resultante experimentado pelo sistema de spins permite o surgimento de componentes transversais (no plano perpendicular ao campo magnético) ou o desaparecimento da componente longitudinal (na direção do campo magnético) desta magnetização.

Após a aplicação dos pulsos de RF, o sistema retorna automaticamente à condição de equilíbrio por meio de mecanismos de relaxações transversal e longitudinal independentes. $\mathrm{O}$ tempo necessário para que a componente transversal desapareça completamente é independente do tempo necessário para que a magnetização longitudinal se reestabeleça. A dinâmica 
dos mecanismos de relaxação da magnetização é descrita fenomenologicamente através das equações de Bloch. (10)

Uma das características da evolução temporal da magnetização durante o processo de relaxação é o movimento de precessão com frequência de Larmor, que é proporcional ao campo magnético e a constante giromagnética, que por sua vez depende apenas de características do núcleo.

Como consequência das equações de Maxwell, a evolução temporal do vetor que representa a magnetização produz ondas eletromagnéticas que oscilam nas mesmas frequências de precessão da magnetização. Portanto, o FID é o sinal detectado a partir da FEM (Força Eletromotriz) induzida pela variação do fluxo magnético no interior das bobinas receptoras como consequência do retorno da magnetização resultante do sistema de spins à condição de equilíbrio. Uma interpretação alternativa para o FID é que este representa uma medida indireta da evolução temporal da dissipação da energia excedente no sistema de spins após a aplicação dos pulsos de RF.

Em uma dada amostra, os mesmos tipos de núcleos podem estar presentes em regiões que contém distribuições eletrônicas distintas ao redor de suas vizinhanças. Portanto, o campo magnético experimentado por estes núcleos é ligeiramente diferente do campo magnético estático $\left(B_{0}\right)$ aplicado. Como consequência deste fenômeno de blindagem, múltiplas ressonâncias podem ser observadas no domínio da frequência para estes mesmos núcleos. A diferença entre a frequência de Larmor para um determinado núcleo e a frequência observada é mensurada em partes por milhão (ppm) e é denominada deslocamento químico (chemical shift).

A intensidade dos picos ou linhas espectrais de cada metabólito no corpo humano é proporcional ao número de prótons visíveis à técnica de $\mathrm{RM}$ e a respectiva concentração molar. (11) A área sob a curva de cada pico no domínio da frequência determina a intensidade do pico. A princípio, este procedimento poderia ser realizado diretamente através da integração numérica no domínio da frequência. No entanto, como a separação entre os picos é proporcional à resolução espectral que, por sua vez, é proporcional ao campo magnético estático, nem sempre este procedimento é aplicável. De fato, em aplicações in vivo os campos magnéticos comumente utilizados são da ordem de $1.5 \mathrm{~T}$ ou $3 \mathrm{~T}$ e, portanto, o grau de sobreposição dos picos impede a determinação da área de cada pico diretamente. Outros fatores importantes são: a taxa de amostragem em que o sinal é adquirido; o acoplamento J (12); e a própria largura linha, ligada às características intrínsecas das moléculas contidas nas amostras. Portanto, tratamentos matemáticos que permitam contornar estes problemas são necessários.

Nas últimas décadas, dois grupos de soluções para o problema de quantificação de metabó- 
litos foram desenvolvidos (13): o ajuste do domínio da frequência através de bases espectrais, que utiliza combinações lineares de sinais individuais de metabólitos adquiridos através de experimentos in vitro ou simulados por meio da teoria da mecânica quântica; e o ajuste através de modelos baseados em funções matemáticas para representar cada componente ou pico do sinal.

$\mathrm{Na}$ abordagem que consiste no ajuste dos picos por meio de funções matemáticas, os métodos são classificados entre aqueles que são processados no domínio da frequência ou no domínio do tempo. Em particular, o ajuste das linhas espectrais por meio de funções matemáticas permite a obtenção de uma lista de parâmetros que descrevem cada pico individualmente, sem a necessidade do uso de conhecimento a priori sobre os picos. Em geral, é assumido que o sinal é constituído por uma somatória de picos ou componentes que podem ser modelados através de linhas espectrais lorentzianas, gaussianas ou através de funções Voigt. Todos esses modelos apresentam vantagens e desvantagens: embora representem a solução física em um cenário sem imperfeições experimentais, os picos lorentzianos não são capazes de representar adequadamente os efeitos do alargamento das linhas; as funções gaussianas, capazes de contornar o problema do alargamento, ignoram os termos de primeira ordem no decaimento exponencial; e as funções Voigt, que combinam as propriedades das funções lorentzianas e gaussianas possuem mais parâmetros a serem determinados, o que pode comprometer o ajuste do sinal. (13-14) Ademais, a robustez à presença de ruído é um problema que vai além da escolha das funções utilizadas no modelo.

Por outro lado, o ajuste utilizando bases espectrais requer uma base específica para cada configuração experimental, que é diferente para cada campo magnético ou sequência de pulsos de RF. Esta abordagem pode se tornar onerosa, levando-se em consideração o alto custo dos compostos químicos necessários em cada experimento, além do tempo necessário na construção da base. Além disso, a abordagem que utiliza a mecânica quântica na simulação das bases requer o tratamento de uma modelagem complexa para cada protocolo de aquisição específico. Ainda assim, em função do seu desempenho, os métodos de ajuste utilizando bases espectrais e informação a priori predominaram na literatura e na prática clínica. (15)

Contudo, algumas tarefas específicas tem sido destinadas à categoria de ajuste através de funções matemáticas, como é o caso da remoção do sinal residual da água que tem sido resolvido pelos métodos baseados no formalismo do espaço de estados. (16-19)

Embora formulados no domínio do tempo, esta família de modelos assume que o espectro pode ser composto através da sobreposição de linhas espectrais de formato lorentziano. O sinal residual da água é um artefato das sequências de pulsos que tentam realizar a supressão total do 
sinal da água durante a aquisição, mas que dificilmente são bem sucedidas, necessitando desta etapa adicional de pós-processamento. Quando este artefato não é removido corretamente, a quantificação pode ser prejudicada. (13)

Desenvolvido fora do contexto das aplicações de ERM in vivo, o Krylov Basis Diagonalization Method (KBDM) compartilha algumas características com os métodos baseados no formalismo de espaço de estados. (20-22) Ambos os métodos utilizam as mesmas funções matemáticas na composição do modelo para representar as linhas espectrais e fazem uso da decomposição em valores singulares das matrizes de sobreposição, construídas a partir dos pontos do sinal no domínio do tempo, em suas implementações mais robustas.

Em relação aos métodos que utilizam o formalismo do espaço de estados, o KBDM possui uma vantagem numérica: todos os parâmetros que caracterizam uma linha espectral lorentziana (amplitude, taxa de relaxação transversal, frequência e fase) são obtidos simultaneamente através da solução de um único problema de autovalores generalizado. Por outro lado, os métodos baseados no formalismo de espaço de estados obtêm as fases e as frequências em um estágio inicial; e uma etapa adicional utilizando o método dos mínimos quadrados é necessária no cálculo das amplitudes e taxas de relaxação transversal. Portanto, erros numéricos associados a este último processo são incorporados às estimativas.

Poucos trabalhos utilizando o KBDM foram publicados na literatura. (23-27) E o conteúdo é ainda mais limitado no contexto da aplicação clínica utilizando ERM, onde podemos referenciar apenas o trabalho realizado em (28). Além disso, dentro do limite do nosso conhecimento, nenhuma comparação do KBDM aos métodos pertencentes àqueles desenvolvidos através do formalismo do espaço de estados foi realizada até o presente momento. Em particular, a comparação com o HLSVD-PRO (Hankel Lanczos Singular Value Decomposition with partial reorthogonalization) (29), que representa o estado da arte entre os algoritmos desta categoria, é necessária para o entendimento das vantagens e limitações do KBDM.

Portanto, este trabalho tem como objetivo estudar e otimizar o desempenho do KBDM nas tarefas associadas ao processamento de sinais de ERM in vivo que não são diretamente impactadas pelo alargamento alargamento de linhas, como é o caso da remoção do sinal residual da água; e a reconstrução de componentes que possam ser adequadamente representadas por linhas lorentzianas em sinais ruidosos. Estes objetivos podem ser enunciados através dos seguintes itens:

1. Implementar o KBDM e realizar um estudo paramétrico: estudar o significado de cada parâmetro interno do método e entender o impacto da escolha dos respectivos valores quando há ruído no sinal e sobreposição das linhas espectrais; 
2. Propor e desenvolver uma técnica que aumente a robustez ao ruído: o método proposto consiste na promediação das estimativas obtidas pelo KBDM por meio de múltiplos truncamentos do mesmo sinal através do agrupamento das linhas espectrais do espaço de parâmetros que caracterizam as componentes lorentzianas. O método desenvolvido recebe o nome Line List Clustering Krylov Basis Diagonalization Method (LLC-KBDM);

3. Viabilizar o uso das técnicas pesquisadas: desenvolver uma ferramenta computacional para uso do KBDM e do LLC-KBDM através de um software multiplataforma com interface gráfica;

4. Situar o desempenho do KBDM e o LLC-KBDM entre os algoritmos da mesma categoria que representam o estado da arte nas aplicações de ERM in vivo: mostrar o desempenho dos algoritmos em sinais simulados e em sinais in vivo.

No Capítulo 2 o Problema da Inversão Harmônica (PIH) é introduzido. Uma breve revisão da literatura sobre os principais métodos que se propõem a resolvê-lo é feita. A descrição matemática da solução do PIH através do KBDM é apresentada com base nos conteúdos disponíveis na literatura. O estudo paramétrico é realizado no final deste capítulo.

No Capítulo 3 uma proposta original para a promediação de múltiplas soluções obtidas através do KBDM para o mesmo sinal utilizando algoritmos de agrupamento é apresentada. O conceito de amostragem e promediação no espaço de parâmetros é proposto. Uma breve introdução sobre os algoritmos de agrupamento de dados é feita. Alguns exemplos são mostrados a partir do processamento de sinais simulados. O pseudo-código e o fluxograma que descrevem o LLC-KBDM são apresentados.

No Capítulo 4 as implementações desenvolvidas durante a execução deste trabalho são discutidas a partir de um ponto de vista tecnológico. O software que permite o uso do algoritmos desenvolvidos por meio de uma interface gráfica é apresentado.

No Capítulo 5 são apresentadas as comparações dos resultados obtidos através dos métodos KBDM, LLC-KBDM e HLSVD-PRO (29) para sinais simulados ruidosos. A demonstração da aplicação do KBDM e o LLC-KBDM na remoção dos sinais residuais da água em dados clínicos também é feita neste capítulo.

No Capítulo 6 são apresentadas as conclusões, as limitações e as sugestões para trabalhos futuros desencadeadas a partir da realização deste projeto de pesquisa. 


\section{Método da Diagonalização na Base de Krylov}

\subsection{Problema da Inversão Harmônica}

Considere o sinal descrito através da série temporal

$$
\begin{gathered}
\tilde{C}_{n}=\sum_{k=0}^{K-1} \tilde{a}_{k} e^{i \widetilde{\Omega}_{k} n \tau} \\
n=0,1, \ldots, N-1
\end{gathered}
$$

onde $N$ é o número de pontos complexos e $K$ é o número de componentes. O tempo decorrido entre duas amostragens consecutivas é $\tau$.

Além disso,

$$
\begin{gathered}
\tilde{a}_{k}=a_{k} e^{i \varphi_{k}} \\
\widetilde{\Omega}_{k}=\omega_{k}+i \gamma_{k}
\end{gathered}
$$

onde $a_{k}, \varphi_{k}, \omega_{k}$ e $\gamma_{k}$ representam, respectivamente, as amplitudes, fases, frequências e constantes de decaimento de cada componente $k=0,1, \ldots, K-1$.

A obtenção da lista de parâmetros $\left\{\left(a_{k}, \varphi_{k}, \omega_{k}, \gamma_{k}\right)\right\}$ a partir do vetor complexo $\tilde{C}$ composto por $N$ amostras da série $\tilde{C}_{n}$ (Equação 2.1) é conhecida como Problema da Inversão Harmônica ou HIP (do inglês, Harmonic Inversion Problem).

Em 1795, em um ensaio sobre as leis da dilatabilidade dos fluidos elásticos e a força expansiva do vapor de álcool a diferentes temperaturas, Barão Gaspard de Prony utilizou uma expressão equivalente à Equação 2.1. (30) Neste mesmo trabalho, Prony propôs a primeira solução conhecida para o Problema da Inversão Harmônica. Este método voltou a receber atenção na literatura somente após o surgimento dos computadores digitais, fruto do desenvolvimento da eletrônica durante o século XX. O método de Prony continuou a ser 
aprimorado e utilizado em diversas aplicações de diferentes domínios, incluindo espectroscopia por RM. (31-34)

Outro método importante na solução do HIP é o Linear Prediction Singular Value Decomposition (LPSVD) desenvolvido por Tufts e Kumaresan. (35) Uma das primeiras tentativas bem sucedidas em resolver o problema do ajuste paramétrico de sinal de ERM in vivo de um rato com um tumor injetado foi realizada através do LPSVD. (36)

Posteriormente, houve notório aperfeiçoamento dos métodos baseados em decomposição em valores singulares. Em especial, a incorporação do formalismo de espaço de estados resultou no método Hankel Singular Value Decomposition (HSVD). (16-17) Embora o HSVD seja muito difundido até os dias de hoje, especialmente em tarefas como a supressão do pico da água de um espectro de RM, este algoritmo apresenta diversas instabilidades numéricas. A performance apresentada nos computadores da época em que foi desenvolvido também era considerada uma limitação do método.

Alguns anos depois, os métodos Hankel Total Least Squares (HTLS), HLSVD with implicitly restarted Lanczos (HLSVD-IRL) e HLSVD with partial reorthogonalization (HLSVDPRO) foram propostos como alternativas que pudessem contornar estas limitações do HSVD. (29, 37-38) Demonstrações sobre a superioridade do HLSVD-PRO foram mostradas. (19)

A Diagonalização Filtrada (DF) é uma outra abordagem na solução do HIP. Embora esta técnica tenha sido desenvolvida no contexto da mecânica quântica, a generalidade de sua aplicação foi observada desde sua primeira publicação. (22) Como sua maior característica, a diagonalização filtrada reduz o HIP a um Problema de Autovalor Generalizado (PAG), onde apenas o intervalo espectral de interesse é obtido. Ou seja, são estimados apenas os parâmetros dos componentes contidos em uma determinada janela de frequências. As ideias fundamentais deste método foram aprimoradas por Mandelshtam et al. em 1997, dando origem ao Método da Diagonalização Filtrada (MDF), também conhecido como FDM (do inglês, Filter Diagonalization Method). (20)

Algumas vantagens do FDM como solução do Problema da Inversão Harmônica são: o seu bom desempenho computacional em sinais contendo muitos pontos, viabilizando o seu uso em $N \approx 10^{4}$; e a existência de sua versão multi-dimensional, que possibilita aplicações em espectroscopia 2D por RM, por exemplo. (39-41) A performance computacional do FDM é um fator importante quando se trata de aplicações de espectroscopia por RM de alta resolução, onde algumas dezenas de milhares de pontos complexos são necessários para representar o sinal.

Em um artigo de revisão sobre o assunto, Mandelshtam concede o nome Krylov Basis 
Diagonalization Method (KBDM) a uma solução "trivial" para o HIP, previamente desenvolvida em (20), que segue o mesmo formalismo do FDM, contudo, sem a utilização da técnica de filtragem. (21) No contexto em que foi elaborado, onde diagonalizações de matrizes muito grandes são necessárias, o KBDM, de fato, não se apresenta como solução superior aos demais métodos existentes para o Problema da Inversão Harmônica. Em outras palavras, neste tipo de aplicação, a eficiência computacional do FDM se torna praticamente obrigatória e, por essa razão, todos os métodos ineficientes neste aspecto são igualmente inapropriados.

No entanto, antes de descartar o KBDM a favor do FDM, algumas peculiaridades deste método devem ser consideradas. A primeira consiste no uso da mesma teoria utilizada no FDM, que garante a existência da solução exata para a lista de parâmetros $\left\{\left(a_{k}, \varphi_{k}, \omega_{k}, \gamma_{k}\right)\right\}$ se a versão discreta da função de correlação temporal em questão satisfizer, também de maneira exata, a Equação 2.1. De fato, a principal diferença entre o FDM e o KBDM é que o FDM utiliza uma base do tipo Fourier; enquanto o KBDM utiliza uma base formada por um subespaço de Krylov. A segunda característica se dá pela simplicidade do KBDM, que requer apenas a solução do Problema de Autovalores Generalizado envolvendo duas matrizes constituídas por pontos do sinal no domínio do tempo. Estes dois motivos por si só já constituem motivação suficiente para analisar alguns cenários onde outros métodos são estabelecidos, mas o KBDM foi pouco explorado.

Em particular, a quantificação de metabólitos através de espectroscopia por RM se dá em equipamentos clínicos ou pré-clínicos, normalmente utilizando-se campos magnéticos de $1.5 \mathrm{~T}$ ou 3.0T. Esta magnitude de campo magnético somada à limitação no tempo de aquisição dos sinais muitas vezes resulta em espectros com pouca resolução e, ainda, de baixa Relação SinalRuído (RSR). Embora a solução ideal deste problema possa ser representada pela Equação 2.1 e a baixa resolução deste tipo de aplicação corrobore com o uso do KBDM, a presença de ruído ainda é um problema a ser contornado.

Assim como ocorre em qualquer outro método de ajuste, quando o sinal não satisfaz exatamente o modelo, o resultado obtido será, na melhor das hipóteses, uma aproximação que dependerá da capacidade do sinal ser representado pelas funções utilizadas no modelo.

Um outro problema comum em aplicações clínicas de RM é o efeito não desprezível do alargamento das linhas espectrais causado pela inomogeneidade do campo magnético estático gerado pelo espectrômetro de RM e por interações internas entre os núcleos vizinhos na amostra. Uma vez que a frequência de ressonância (frequência de Larmor) de cada núcleo é proporcional à magnitude do campo magnético estático do sistema, $B_{0}$, as imperfeições do magneto e a interação do campo magnético com a amostra ou paciente podem causar o 
aparecimento de termos de segunda ordem nas componentes do sinal no domínio do tempo, que se manifestam através de alargamentos dos picos no domínio da frequência.

\subsection{A conexão entre o Problema da Inversão Harmônica e um sistema dinâmico fictício}

A notação matemática e os formalismos adotados originalmente na literatura sobre o método a ser discutido a partir desta seção não são os mais adequados para o contexto da aplicação deste trabalho. Mesmo após a sua introdução às aplicações em espectroscopia por ressonância magnética, os principais trabalhos relacionados ao tema mantiveram o formalismo e utilizaram uma versão adaptada da notação de Dirac para sistemas quânticos dissipativos. Portanto, a partir desta seção (incluindo Seções 2.3 e 2.4), serão discutidos os métodos originalmente descritos em (20-22) através da notação matricial complexa utilizada em (26). Acreditamos que esta decisão simplifica a leitura e entendimento do método, abandonando os significados físicos da notação originalmente proposta, que poderia, inclusive, causar interpretações inadequadas.

Considere um sistema dinâmico fictício descrito pela equação

$$
\frac{d \tilde{X}(t)}{d t}=i \tilde{W} \tilde{X}(t)
$$

com respectiva solução dada por

$$
\tilde{X}(t)=e^{i \tilde{W} t} \tilde{X}(0)
$$

onde $\tilde{X}(t) \in \mathbb{C}^{K}$ é um vetor propagado a partir de sua condição inicial $\tilde{X}(0)$ e $\tilde{W}$ é um operador definido a partir de uma matriz simétrica complexa, tal que

$$
\begin{gathered}
\tilde{W} \in \mathbb{C}^{K \times K} \\
\tilde{W}=\tilde{W}^{T}
\end{gathered}
$$

A simetria deste operador possui papel importante: em contraste a um operador hermitiano, que possui simetria complexa conjugada e, portanto, é um operador unitário, $\tilde{W}$ não oferece a propriedade da preservação da norma de um dado vetor $\tilde{v} \in \mathbb{C}^{K \times 1}$ na operação $\tilde{W} \tilde{v}$. Esta é uma característica fundamental dos sistemas dissipativos, que se adequa perfeitamente ao comportamento representado pelo decaimento exponencial das componentes contidas na Equação 2.1. Desta forma, é conveniente definir o produto interno entre dois vetores $\tilde{u}, \tilde{v} \in \mathbb{A}$, 
sendo $\mathbb{A}$ o espaço onde estão representados $\tilde{W}$ e $\tilde{X(t)}$, por

$$
<\tilde{u}, \tilde{v}>=\tilde{u}^{T} \tilde{v}=\tilde{v}^{T} \tilde{u}
$$

onde a transposição ordinária é utilizada no lugar da transposição conjugada.

É importante notar que esse produto interno pode resultar em valores reais negativos e, portanto, não permite a definição da norma de um vetor no espaço $\mathbb{A}$ que, como consequência, não pode ser considerado um espaço métrico.

Definimos agora a função de autocorrelação temporal

$$
\tilde{c}(t)=\tilde{X}^{T}(0) \tilde{X}(t)
$$

que pode ser reescrita em termos da Equação 2.6

$$
\tilde{c}(t)=\tilde{X}^{T}(0) e^{i \tilde{W} t} \tilde{X}(0)
$$

Para evitar complicações desnecessárias, vamos assumir que $\tilde{W}$ é diagonalizável. Além disso, vamos omitir a existência de degenerescência em seus autovalores, de modo que

$$
\begin{gathered}
\tilde{W} \tilde{V}_{k}=\tilde{\Omega}_{k} \tilde{V}_{k} \\
k=0,1, \ldots, K-1
\end{gathered}
$$

sendo $\tilde{V}_{k} \in \mathbb{C}^{K}$ o $k$-ésimo autovetor de $\tilde{W}$ e $\widetilde{\Omega}_{k} \in \mathbb{C}$ seu respectivo autovalor, com $\widetilde{\Omega}_{k} \neq \widetilde{\Omega}_{k^{\prime}}$ se $k \neq k^{\prime}$. Além disso, a ortogonalidade e as normalizações destes autovetores devem ser garantidas de maneira que

$$
\tilde{V}_{k}^{T} \tilde{V}_{k^{\prime}}=\delta_{k k^{\prime}}
$$

onde $\delta_{k k^{\prime}}$ é o delta de Kronecker.

A Equação 2.12 pode ser reescrita de uma forma compacta para todos os autovalores e autovetores de $\tilde{W}$

$$
\tilde{W} \tilde{V}=\tilde{V} \widetilde{\Omega}
$$

onde $\tilde{V} \in \mathbb{C}^{K \times K}$ é uma matriz que contém os autovetores $\tilde{V}_{k}$ dispostos em suas colunas

$$
\tilde{V}=\left[\begin{array}{cccc}
\uparrow & \uparrow & \cdots & \uparrow \\
\tilde{V}_{0} & \tilde{V}_{1} & \cdots & \tilde{V}_{K-1} \\
\downarrow & \downarrow & \cdots & \downarrow
\end{array}\right]
$$


e $\widetilde{\Omega}$ é uma matriz diagonal contendo os autovalores complexos, $\Omega_{i}$, tal que

$$
\widetilde{\Omega}=\left[\begin{array}{cccc}
\widetilde{\Omega}_{0} & 0 & \cdots & 0 \\
0 & \widetilde{\Omega}_{1} & \cdots & 0 \\
\vdots & \vdots & \ddots & \vdots \\
0 & 0 & \cdots & \widetilde{\Omega}_{K-1}
\end{array}\right]
$$

Como consequência da ortogonalidade e normalização dos autovetores de $\tilde{W}$ (Equação 2.14)

$$
\tilde{V}^{T} \tilde{V}=\mathbf{I} \Longrightarrow \tilde{V}^{-1}=\tilde{V}^{T}
$$

Ou seja, $\tilde{V}$ é uma matriz ortogonal e sua inversa pode ser diretamente obtida a partir da operação de transposição. A ortogonalidade de $\tilde{V}$, mesmo quando $\tilde{W}$ é complexo, é uma consequência da definição do produto interno (Equação 2.9) no espaço $\mathbb{A}$. Este fato deve ser observado para que não haja confusão com a decomposição espectral no espaço de Hilbert, onde $\tilde{V}$ seria unitário, ao invés de ortogonal.

Portanto, a decomposição espectral do operador $\tilde{W}$ se dá por

$$
\tilde{W}=\tilde{V} \widetilde{\Omega} \tilde{V}^{-1}=\tilde{V} \widetilde{\Omega} \tilde{V}^{T}
$$

Como consequência imediata, a Equação 2.11 pode ser reescrita em função dos autovalores e autovalores de $\tilde{W}$

$$
\tilde{c}(t)=\tilde{X}^{T}(0) \tilde{V} e^{i \widetilde{\Omega} t} \tilde{V}^{T} \tilde{X}(0)
$$

Explorando a ortogonalidade de $\tilde{V}$, a seguinte propriedade matricial foi aplicada: quando $\alpha$ é invertível,

$$
e^{\alpha \beta \alpha^{-1}}=\alpha e^{\beta} \alpha^{-1}
$$


Seja $\tilde{Y} \in \mathbb{C}^{K \times 1}$ o vetor coluna

$$
\begin{gathered}
\tilde{Y}=\tilde{V}^{T} \tilde{X}(0) \\
\tilde{Y}=\left[\begin{array}{c}
\tilde{y}_{0} \\
\tilde{y}_{1} \\
\vdots \\
\\
\tilde{y}_{K-1}
\end{array}\right] \\
\tilde{y}_{k}=\tilde{V}_{k}^{T} \tilde{X}(0)
\end{gathered}
$$

e $\tilde{Y}^{T} \in \mathbb{C}^{1 \times K}$ o vetor linha

$$
\tilde{Y}^{T}=\left(\tilde{V}^{T} \tilde{X}(0)\right)^{T}=\tilde{X}^{T}(0) \tilde{V}
$$

é possível rescrever a Equação 2.10 da seguinte forma

$$
\begin{aligned}
\tilde{c}(t) & =\tilde{Y}^{T}(t) e^{i \widetilde{\Omega}_{k} t} \tilde{Y}(t) \\
& =\sum_{k=0}^{K-1} \tilde{y}_{k}^{2} e^{i \widetilde{\Omega}_{k} t}
\end{aligned}
$$

onde

$$
\tilde{y}_{k}^{2} \equiv \tilde{a}_{k}
$$

E finalmente obtemos

$$
\tilde{c}(t)=\sum_{k=0}^{K-1} a_{k} e^{i \widetilde{\Omega}_{k} t}
$$

Partindo das definições dadas pelas das Equações 2.3 e 2.4,

$$
\tilde{c}(t)=\sum_{k=0}^{K-1} a_{k} e^{-\gamma_{k} t} e^{i\left(\omega_{k} t+\varphi_{k}\right)}
$$

As Equações 2.28 e 2.29 nada mais são do que a versões contínuas da série temporal definida pela Equação 2.1. Ou seja,

$$
\tilde{c}(t=n \tau) \rightarrow \tilde{C}_{n}
$$

Consequentemente, podemos concluir que a lista de parâmetros $\left\{\left(\tilde{a}_{k}, \widetilde{\Omega}_{k}\right)\right\}$ ou, equivalentemente, $\left\{\left(a_{k}, \varphi_{k}, \omega_{k}, \gamma_{k}\right)\right\}$ está intrinsecamente ligada aos autovalores e autovetores do operador $\tilde{W}$, que é responsável pela evolução temporal do sistema dinâmico fictício descrito 
pela Equação 2.5.

Do ponto de vista prático, embora interessante, essa observação não acrescenta muitas vantagens quando há conhecimento apenas sobre $\tilde{C}_{n}$ e nada se sabe sobre o operador $\tilde{W}$ ou mesmo sobre o vetor propagado $\tilde{X}(t)$, incluindo sua respectiva condição inicial $\tilde{X}(0)$.

\subsection{O KBDM como solução do HIP}

Consideremos a partir de agora apenas a versão discreta do sistema discutido anteriormente. Isto é,

$$
\begin{gathered}
t \rightarrow n \tau \\
\tilde{X(t)} \rightarrow \tilde{X}_{n} \\
\tilde{c}(t) \rightarrow \tilde{C}_{n}=\tilde{X}_{0}^{T} \tilde{X}_{n} \\
n=0,1, \ldots, N-1
\end{gathered}
$$

Seja o operador de evolução temporal

$$
\begin{gathered}
\tilde{\mathcal{U}}=e^{i \tilde{W} \tau} \\
\tilde{\mathcal{U}} \in \mathbb{C}^{M \times M}
\end{gathered}
$$

que ao ser aplicado no vetor propagado $\tilde{X}_{n}$ resulta em

$$
\tilde{\mathcal{U}} \tilde{X}_{n}=\tilde{X}_{n+1}
$$

Ou ainda,

$$
\tilde{\mathcal{U}}^{p} \tilde{X}_{n}=\tilde{X}_{n+p}
$$

Além disso, a partir da decomposição espectral de $\tilde{W}$ e da propriedade dada pela Equação 2.21

$$
\begin{gathered}
\tilde{\mathcal{U}}=e^{\tilde{V} i \tilde{\Omega} \tau \tilde{V}^{-1}}=\tilde{V} e^{i \widetilde{\Omega} \tau} \tilde{V}^{-1} \\
\tilde{\mathcal{U}} \tilde{V}=\tilde{V} e^{i \widetilde{\Omega} \tau}=\tilde{V} \tilde{\mu}
\end{gathered}
$$

onde cada elemento da matriz diagonal $\tilde{\mu} \in \mathbb{C}^{K \times K}$ é dado por

$$
\tilde{\mu}_{k}=e^{\widetilde{\Omega}_{k} \tau}
$$


Portanto, além da mesma simetria, $\tilde{\mathcal{U}}$ e $\tilde{W}$ também compartilham os mesmos autovetores $\tilde{V}_{k}$, tal que

$$
\tilde{\mathcal{U}} \tilde{V}_{k}=\tilde{\mu}_{k} \tilde{V}_{k}
$$

e seus autovalores são diretamente relacionados pela Equação 2.41.

Resolver a Equação 2.42 é equivalente a resolver a Equação 2.12, mas até este ponto não há informação suficiente para tal tarefa. O que será mostrado a seguir é que, através de uma mudança apropriada de base, este problema se reduzirá à solução de um problema de autovetores generalizado. Para elaborar o problema de maneira genérica antes de escolher uma base específica, a existência de uma base $\tilde{\Phi}$ é assumida

$$
\begin{gathered}
\tilde{\Phi}=\left\{\tilde{\Phi}_{n}\right\} \\
\tilde{\Phi}_{n} \in \mathbb{C}^{K} \\
n=0, \ldots, M-1
\end{gathered}
$$

onde $M$ é número de vetores que compõem esta base.

O próximo passo consiste em escrever os vetores $\tilde{V}_{k}$ através de combinações lineares dos vetores $\tilde{\Phi}_{n}$ que formam esta nova base

$$
\tilde{V}_{k}=\sum_{n=0}^{M-1} \tilde{B}_{n, k} \tilde{\Phi}_{n}
$$

onde $\tilde{B}_{n, k} \in \mathbb{C}$ são os elementos do vetores $\tilde{B}_{k} \in \mathbb{C}^{M}$ que compõem cada coluna da matriz $\tilde{B} \in \mathbb{C}^{M \times K}$ :

$$
\tilde{B}=\left[\begin{array}{cccc}
\tilde{B}_{0,0} & \tilde{B}_{0,1} & \cdots & \tilde{B}_{0, K-1} \\
\tilde{B}_{1,0} & \tilde{B}_{1,1} & \cdots & \tilde{B}_{1, K-1} \\
\vdots & \vdots & \ddots & \vdots \\
\tilde{B}_{M-1,0} & \tilde{B}_{M-1,1} & \cdots & \tilde{B}_{M-1, K-1}
\end{array}\right]
$$

Aplicando-se a transformação de base dada pela Equação 2.46 ao problema de autovalores para o operador de evolução temporal (Equação 2.42) e multiplicando a expressão resultante por $\tilde{\Phi}_{n^{\prime}}^{T}$ à esquerda em ambos os membros

$$
\begin{aligned}
\tilde{\Phi}_{n^{\prime}}^{T} \tilde{\mathcal{U}}^{p} \sum_{n=0}^{M-1} \tilde{B}_{n, k} \tilde{\Phi}_{n} & =\tilde{\Phi}_{n^{\prime}}^{T} \tilde{\mu}_{k}^{p} \sum_{n=0}^{M-1} \tilde{B}_{n, k} \tilde{\Phi}_{n} \\
\sum_{n=0}^{M-1}\left(\tilde{\Phi}_{n^{\prime}}^{T} \tilde{\mathcal{U}}^{p} \tilde{\Phi}_{n}\right) \tilde{B}_{n, k} & =\tilde{\mu}_{k}^{p} \sum_{n=0}^{M-1}\left(\tilde{\Phi}_{n^{\prime}}^{T} \tilde{\Phi}_{n}\right) \tilde{B}_{n, k}
\end{aligned}
$$


Estas somas podem ser interpretadas como multiplicações das matrizes $\tilde{U}^{p}$ (membro esquerdo) e $\tilde{S}$ (membro direito) pelos vetores $\tilde{B}_{k}$

$$
\tilde{U}^{p} \tilde{B}_{k}=\tilde{\mu}_{k}^{p} \tilde{S} \tilde{B}_{k}
$$

com os respectivos elementos

$$
\begin{gathered}
\tilde{U}_{n, m}^{p}=\tilde{\Phi}_{n}^{T} \tilde{\mathcal{U}}^{p} \tilde{\Phi}_{m} \\
\tilde{S}_{n, m}=\tilde{\Phi}_{n}^{T} \tilde{\Phi}_{m}=\tilde{\Phi}_{n}^{T} \tilde{\mathcal{U}}^{0} \tilde{\Phi}_{m}=\tilde{U}_{n, m}^{0}
\end{gathered}
$$

Todas essas matrizes representam o operador $\tilde{\mathcal{U}}$ e suas potências na base $\tilde{\Phi}$. Em particular, quando $p=0$, a matriz $\tilde{U}^{p}$ se reduz a matriz $\tilde{S}=\tilde{U}^{0}$, denominada por matriz de sobreposição. Daqui em diante, apenas o símbolo $\tilde{U}^{0}$ será utilizado para denotar a matriz de sobreposição.

Para concluir o procedimento, resta ainda encontrar uma base que permita o cálculo dos elementos $\tilde{U}_{n, m}^{p}$; resolver o PAG descrito pela Equação 2.50; e obter as amplitudes $\tilde{a}_{k}$ a partir dos autovetores $\tilde{B}_{k}$.

O uso combinado do operador $\tilde{U}$ e dos autovetores propagados $\tilde{X}_{n}$ se faz necessário para que a informação contida em $\tilde{C}_{n}$ seja utilizada. Utilizando-se potências do operador de evolução é possível escrever um vetor propagado $\tilde{X}_{n}$ a partir de seu estado inicial $\tilde{X}_{0}$, tal que

$$
\tilde{U}^{n} \tilde{X}_{0}=\tilde{X}_{n}
$$

onde $\tilde{X}_{n}$ é um elemento contido no subespaço definido por

$$
\mathcal{K}_{M}\left(\tilde{U}, \tilde{X}_{0}\right)=\operatorname{span}\left\{\tilde{X}_{0}, \tilde{U} \tilde{X}_{0}, \tilde{U}^{2} \tilde{X}_{0}, \ldots, \tilde{U}^{M-1} \tilde{X}_{0}\right\}
$$

$\mathcal{K}_{M}\left(\tilde{U}, \tilde{X}_{0}\right)$ é denominado por subespaço de Krylov de ordem $M$ gerado pelas potências do operador $\tilde{U}$ aplicadas sobre o vetor $\tilde{X}_{0}$.

Os elementos que compõem o subespaço $\mathcal{K}_{M}\left(\tilde{U}, \tilde{X}_{0}\right)$ são, portanto, a escolha natural para a base $\tilde{\Phi}$. Deste modo, quando

$$
\tilde{\Phi}_{n}=\tilde{X}_{n}=\tilde{U}^{n} \tilde{X}_{0}
$$

o método abordado nesta seção recebe o nome de Método da Diagonalização da Base de Krylov, originalmente denominado em inglês por Krylov Basis Diagonalization Method (KBDM).

Basta agora calcular os elementos $\tilde{U}_{n, m}^{p}$ e $\tilde{\mathbf{S}}_{n, m}^{p}$ utilizando esta base. Para isso, considere 
a transposição de ambos os membros da Equação 2.53

$$
\begin{aligned}
& \left(U^{n} \tilde{X}_{0}\right)^{T}=\tilde{X}_{n}^{T} \\
& \tilde{X}_{0}^{T}\left(U^{n}\right)^{T}=\tilde{X}_{n}^{T}
\end{aligned}
$$

onde a simetria $U^{n}=\left(U^{n}\right)^{T}$ pode ser aplicada de maneira que

$$
\tilde{X}_{0}^{T} \tilde{U}^{n}=\tilde{X}_{n}^{T}
$$

Multiplicando por $\tilde{X}_{0}$ à esquerda

$$
\tilde{X}_{0}^{T} U^{n} \tilde{X}_{0}=\tilde{X}_{n}^{T} \tilde{X}_{0}=\tilde{C}_{n}
$$

Com este artifício, finalmente é possível computar os elementos

$$
\begin{gathered}
\tilde{U}_{n, m}^{p}=\tilde{X}_{n}^{T} \tilde{U}^{p} \tilde{X}_{m}=\tilde{X}_{0}^{T} U^{n+m+p} \tilde{X}_{0}=\tilde{C}_{n+m+p} \\
\tilde{U}_{n, m}^{0}=\tilde{C}_{n+m}
\end{gathered}
$$

Portanto, a estrutura destas matrizes é dada por

$$
\tilde{U}^{0}=\left[\begin{array}{cccc}
\tilde{C}_{0} & \tilde{C}_{1} & \cdots & \tilde{C}_{M-1} \\
\tilde{C}_{1} & \tilde{C}_{2} & \cdots & \tilde{C}_{M} \\
\vdots & \vdots & \ddots & \vdots \\
\tilde{C}_{M-1} & \tilde{C}_{M} & \cdots & \tilde{C}_{2 M-2}
\end{array}\right]
$$

e

$$
\tilde{U}^{p}=\left[\begin{array}{cccc}
\tilde{C}_{p} & \tilde{C}_{1+p} & \cdots & \tilde{C}_{M-1+p} \\
\tilde{C}_{1+p} & \tilde{C}_{2+p} & \cdots & \tilde{C}_{M+p} \\
\vdots & \vdots & \ddots & \vdots \\
\tilde{C}_{M-1+p} & \tilde{C}_{M+p} & \cdots & \tilde{C}_{2 M-2+p}
\end{array}\right]
$$

A partir das Equações 2.62 e 2.63 é possível observar que as matrizes $\tilde{U}^{0}$ e $\tilde{U}^{p}$ possuem 
uma estrutura especial. Uma propriedade interessante entre seus elementos é dada por

$$
\begin{gathered}
\tilde{U}_{n, m}^{p}=\tilde{U}_{n+k, m-k}^{p} \\
k=0,1, \ldots, m-n
\end{gathered}
$$

Matrizes que possuem a simetria dada pela Equação 2.64 são denominadas matrizes de Hankel.

Quando $p=1$, a Equação 2.50 se torna

$$
\tilde{U}^{1} \tilde{B}_{k}=\tilde{\mu}_{k} \tilde{U}^{0} \tilde{B}_{k}
$$

e sua solução será tema da sessão 2.4. Por ora, será assumido o conhecimento de $\tilde{B}_{k}$ e $\tilde{\mu}_{k}$, independente do método utilizado para obtê-los.

Os autovetores $\tilde{B}_{k}$ precisam ser normalizados para que a seguinte condição seja satisfeita:

$$
\tilde{B}_{k}^{T} \tilde{U}^{0} \tilde{B}_{k}=1
$$

e os valores de $\widetilde{\Omega}_{k}$ podem ser diretamente obtidos a partir dos autovalores $\tilde{\mu}_{k}$ (Equação 2.41 )

$$
\widetilde{\Omega}_{k}=\frac{\ln \left(\tilde{\mu}_{k}\right)}{i \tau}
$$

que permitem o cálculo das frequências $\omega_{k}$ e dos fatores de amortecimento $\gamma_{k}$

$$
\begin{aligned}
\omega_{k} & =\operatorname{Re}\left\{\widetilde{\Omega}_{k}\right\} \\
\gamma_{k} & =\operatorname{Im}\left\{\widetilde{\Omega}_{k}\right\}
\end{aligned}
$$

A Equação 2.25 combinada à Equação 2.46 resulta em

$$
\begin{gathered}
\tilde{y}_{k}=\tilde{V}_{k}^{T} \tilde{X}_{0}=\tilde{X}_{0}^{T} \tilde{V}_{k} \\
\tilde{y}_{k}=\sum_{n=0}^{M-1} \tilde{B}_{n, k} \tilde{X}_{0}^{T} \tilde{X}_{n}=\sum_{n=0}^{M-1} \tilde{B}_{n, k} \tilde{C}_{n} \\
\tilde{y}_{k}=\tilde{B}_{k}^{T}[\tilde{C}]_{M}
\end{gathered}
$$

Onde $[\tilde{C}]_{M}$ é o vetor coluna $\tilde{C}_{n}$ truncado em apenas $M$ pontos complexos. 
Portanto,

$$
\begin{gathered}
\tilde{a}_{k}=\tilde{y}_{k}^{2}=\left(\tilde{B}_{k}^{T}[\tilde{C}]_{M}\right)^{2} \\
\tilde{a}_{k}=a_{k} e^{i \varphi_{k}} \\
a_{k}=\left|\tilde{a}_{k}\right| \\
\varphi_{k}=\arg \left(\tilde{a}_{k}\right)
\end{gathered}
$$

\subsection{Solução do Problema de Autovalores Generalizado}

\subsubsection{Exemplo analítico}

Para verificação da teoria do KBDM através de um exemplo simplificado, será considerado a seguir um sinal $\tilde{C}_{n}$ com apenas duas componentes $(K=2)$

$$
\tilde{C}_{n}=\tilde{a}_{1} e^{i \widetilde{\Omega}_{1} n \tau}+\tilde{a}_{2} e^{\widetilde{\Omega}_{2} n \tau}
$$

A dimensionalidade das matrizes $\tilde{U}^{0}$ e $\tilde{U}^{1}$ será definida aqui de maneira a garantir a não singularidade das mesmas. Ou seja, $M=K=2$.

$$
\begin{gathered}
\tilde{U}^{0}=\left[\begin{array}{ll}
\tilde{C}_{0} & \tilde{C}_{1} \\
\tilde{C}_{1} & \tilde{C}_{2}
\end{array}\right] \\
\tilde{U}^{1}=\left[\begin{array}{ll}
\tilde{C}_{1} & \tilde{C}_{2} \\
\tilde{C}_{2} & \tilde{C}_{3}
\end{array}\right]
\end{gathered}
$$

onde

$$
\begin{gathered}
\tilde{C}_{0}=\tilde{a}_{1}+\tilde{a}_{2} \\
\tilde{C}_{1}=\tilde{a}_{1} e^{i \widetilde{\Omega}_{1} \tau}+\tilde{a}_{2} e^{i \widetilde{\Omega}_{2} \tau}=\tilde{a}_{1} \widetilde{\mu}_{1}+\tilde{a}_{2} \widetilde{\mu}_{2} \\
\tilde{C}_{2}=\tilde{a}_{1} e^{2 i \widetilde{\Omega}_{1} \tau}+\tilde{a}_{2} e^{2 i \widetilde{\Omega}_{2} \tau}=\tilde{a}_{1} \widetilde{\mu}_{1}^{2}+\tilde{a}_{2} \widetilde{\mu}_{2}^{2} \\
\tilde{C}_{3}=\tilde{a}_{1} e^{3 i \widetilde{\Omega}_{1} \tau}+\tilde{a}_{2} e^{3 i \widetilde{\Omega}_{2} \tau}=\tilde{a}_{1} \widetilde{\mu}_{1}^{3}+\tilde{a}_{2} \widetilde{\mu}_{2}^{3}
\end{gathered}
$$


Reescrevendo a 2.66 da seguinte maneira

$$
\left(\tilde{U}^{1}-\tilde{\mu}_{k} \tilde{U}^{0}\right) \tilde{B}_{k}=0
$$

é possível observar que as soluções estão contidas no espaço nulo da matriz $\tilde{U}^{1}-\tilde{\mu}_{k} \tilde{U}^{0}$. Os autovalores $\tilde{\mu}_{k}$ são as soluções da equação secular

$$
\begin{gathered}
P\left(\tilde{\mu}_{k}\right)=\operatorname{det}\left(\tilde{U}^{1}-\tilde{\mu}_{k} \tilde{U}^{0}\right)=0 \\
\operatorname{det}\left[\begin{array}{ll}
\tilde{C}_{1}-\tilde{\mu}_{k} \tilde{C}_{0} & \tilde{C}_{2}-\tilde{\mu}_{k} \tilde{C}_{1} \\
\tilde{C}_{2}-\tilde{\mu}_{k} \tilde{C}_{1} & \tilde{C}_{3}-\tilde{\mu}_{k} \tilde{C}_{2}
\end{array}\right]=0 \\
P\left(\tilde{\mu}_{k}\right)=\left(\tilde{C}_{1}-\tilde{\mu}_{k} \tilde{C}_{0}\right)\left(\tilde{C}_{3}-\tilde{\mu}_{k} \tilde{C}_{2}\right)-\left(\tilde{C}_{2}-\tilde{\mu}_{k} \tilde{C}_{1}\right)^{2}=0
\end{gathered}
$$

onde $P\left(\tilde{\mu}_{k}\right)$ é um polinômio de grau $M=2$ neste exemplo.

Após algum trabalho algébrico, é possível verificar que $\tilde{\mu}_{1}=e^{i \widetilde{\Omega}_{1} \tau}$ e $\tilde{\mu}_{2}=e^{i \widetilde{\Omega}_{2} \tau}$ são soluções da Equação 2.88. São associados a estes autovalores, respectivamente, os seguintes autovetores

$$
\tilde{B}_{1}=\left[\begin{array}{c}
1 \\
-1 / \tilde{\mu}_{2}
\end{array}\right]
$$

e

$$
\tilde{B}_{2}=\left[\begin{array}{c}
1 \\
-1 / \tilde{\mu}_{1}
\end{array}\right]
$$

que após processo de normalização (Equação 2.67) se tornam

$$
\begin{gathered}
\tilde{B}_{1}^{\prime}=\left[\begin{array}{c}
\frac{\mu_{2}}{\sqrt{\tilde{a}_{1}\left(\mu_{2}-\mu_{1}\right)^{2}}} \\
-\frac{1}{\sqrt{\tilde{a}_{1}\left(\mu_{2}-\mu_{1}\right)^{2}}}
\end{array}\right] \\
\tilde{B}_{2}^{\prime}=\left[\begin{array}{c}
\frac{\mu_{1}}{\sqrt{\tilde{a}_{2}\left(\mu_{1}-\mu_{2}\right)^{2}}} \\
-\frac{1}{\sqrt{\tilde{a}_{2}\left(\mu_{1}-\mu_{2}\right)^{2}}}
\end{array}\right]
\end{gathered}
$$


e satisfazem as igualdades

$$
\begin{gathered}
\tilde{U}^{1} \tilde{B}_{1}^{\prime}=\tilde{\mu}_{1} \tilde{U}^{0} \tilde{B}_{1}^{\prime} \\
\tilde{U}^{1} \tilde{B}_{2}^{\prime}=\tilde{\mu}_{2} \tilde{U}^{0} \tilde{B}_{2}^{\prime} \\
\tilde{B}_{1}^{\prime T} \tilde{U}^{0} \tilde{B}_{1}^{\prime}=1 \\
\tilde{B}_{2}^{\prime T} \tilde{U}^{0} \tilde{B}_{2}^{\prime}=1 \\
\tilde{B}_{1}^{\prime T} \tilde{U}^{0} \tilde{B}_{2}^{\prime}=\tilde{B}_{2}^{\prime T} \tilde{U}^{0} \tilde{B}_{1}^{\prime}=0
\end{gathered}
$$

Deste modo, os autovetores estão adequadamente definidos para que o cálculo das amplitudes seja realizado através da Equação 2.74. É possível verificar que

$$
\begin{aligned}
& \tilde{a}_{1}=\left(\tilde{B}_{1}^{\prime T}[\tilde{C}]_{2}\right)^{2} \\
& \tilde{a}_{2}=\left(\tilde{B}_{2}^{\prime T}[\tilde{C}]_{2}\right)^{2}
\end{aligned}
$$

onde $[\tilde{C}]_{2}$ é um vetor coluna contendo os $M=2$ primeiros pontos do sinal definido pela Equação 2.78, tal que

$$
[\tilde{C}]_{2}=\left[\begin{array}{c}
\tilde{C}_{0} \\
\tilde{C}_{1}
\end{array}\right]
$$

Portanto, fica concluída a verificação analítica do KBDM para o exemplo contendo duas componentes. Foram necessários apenas 4 pontos complexos do sinal $\left(\tilde{C}_{0}, \tilde{C}_{1}, \tilde{C}_{2}\right.$ e $\left.\tilde{C}_{3}\right)$ no cálculo das matrizes $\tilde{U}^{0}$ e $\tilde{U}^{1}$, resultando na reconstrução completa do sinal, que digitalmente pode ser realizada em resolução limitada apenas pela representação numérica em ponto flutuante.

Se o mesmo sinal for resolvido através do KBDM com apenas dois pontos $(N=2 \mathrm{e}$ $M=1$ ), apenas um autovalor e seu respectivo autovetor serão calculados:

$$
\begin{gathered}
\tilde{\mu}_{1}=\frac{\tilde{C}_{1}}{\tilde{C}_{0}}=\frac{\tilde{\mu}_{1} \tilde{a}_{1}+\tilde{\mu}_{2} \tilde{a}_{2}}{\tilde{a}_{1}+\tilde{a}_{2}} \\
\tilde{B}_{1}=\frac{1}{\sqrt{\tilde{C}_{0}}}
\end{gathered}
$$

resultando em

$$
\begin{gathered}
\tilde{\Omega}_{1}=\frac{\ln \left(\tilde{\mu}_{1}\right)}{i \tau} \\
\tilde{a}_{1}=\left(\tilde{B}_{1}^{\prime T}[\tilde{C}]_{1}\right)^{2}=\left(\frac{\tilde{C}_{0}}{\sqrt{\tilde{C}_{0}}}\right)^{2}=\tilde{a}_{1}+\tilde{a}_{2}
\end{gathered}
$$


O sinal resultante, portanto, não é exatamente igual ao sinal de verdadeiro, conforme o esperado. Entretanto, é interessante realizar algumas observações. O sinal resultante possui uma única componente cuja frequência é associada a um autovalor caracterizado pela média ponderada nas amplitudes dos autovalores verdadeiros. Além disso, quando $\tilde{\mu}_{1} \rightarrow \tilde{\mu}_{2}$, o sinal estimado se torna o sinal verdadeiro. Deste modo, o resultado obtido pode ser interpretado como uma estimativa de baixa resolução quando as frequências do sinal são próximas.

Quando $M>K$, um outro problema é encontrado durante obtenção dos autovalores e autovetores generalizados: o sistema que descreve o PAG dado pela Equação 2.66 se torna sobre-determinado. Algumas consequências desta afirmação são válidas: o sistema agora possui infinitas soluções; e os autovalores possuem degenerescência, isto é, existem autovalores que se repetem. Neste caso, o problema está malposto.

\subsubsection{Decomposição em valores singulares}

A fatoração da matriz $\tilde{U}^{0}$ pode ser utilizada na solução da Equação 2.66. Entre as possíveis abordagens, a decomposição em valores singulares, também conhecida por Singular Value Decomposition (SVD) é realizada da seguinte maneira

$$
\begin{gathered}
\tilde{U}^{0}=\tilde{L} D \tilde{R}^{H} \\
\tilde{L}, \tilde{R} \in \mathbb{C}^{M \times J} \\
D \in \mathbb{R}^{J \times J} \\
J, M \in \mathbb{N} \\
J \leq M
\end{gathered}
$$

onde o símbolo $H$ denota o operador Hermitiano. De fato, a decomposição em valores singulares pode ser aplicada em matrizes retangulares. Entretanto, como $\tilde{U}^{0}$ é necessariamente quadrada, esta generalidade não será explorada. 
$D$ é uma matriz diagonal e todos seus elementos são reais

$$
\begin{gathered}
\widetilde{D}=\left[\begin{array}{cccc}
d_{0} & 0 & \cdots & 0 \\
0 & d_{1} & \cdots & 0 \\
\vdots & \vdots & \ddots & \vdots \\
0 & 0 & \cdots & d_{J-1}
\end{array}\right] \\
d_{0}>d_{1}>\ldots>d_{J-1} \\
d_{j} \geq 0 \\
d_{j} \in \mathbb{R}
\end{gathered}
$$

As matrizes $\tilde{L}$ e $\tilde{R}$, conhecidas como vetores singulares à esquerda e à direita, respectivamente, são matrizes unitárias, tal que

$$
\begin{aligned}
& \tilde{L} \tilde{L}^{H}=\tilde{L}^{H} \tilde{L}=\mathbf{I} \Longrightarrow \tilde{L}^{-1}=\tilde{L}^{H} \\
& \tilde{R} \tilde{R}^{H}=\tilde{R}^{H} \tilde{R}=\mathbf{I} \Longrightarrow \tilde{R}^{-1}=\tilde{R}^{H}
\end{aligned}
$$

O vetor singular à esquerda, $\tilde{L}$, é composto pelos autovetores da matriz $\tilde{U}^{0}\left(\tilde{U}^{0}\right)^{H}$

$$
\begin{aligned}
\tilde{U}^{0}\left(\tilde{U}^{0}\right)^{H} & =\tilde{L} D \tilde{R}^{H}\left(\tilde{L} D \tilde{R}^{H}\right)^{H} \\
& =\tilde{L} D\left(\tilde{R}^{H} \tilde{R}\right) D \tilde{L}^{H} \\
& =\tilde{L} D^{2} \tilde{L}^{H}
\end{aligned}
$$

enquanto que os autovetores da matriz $\left(\tilde{U}^{0}\right)^{H} \tilde{U}^{0}$, de maneira análoga, são colunas do vetor singular à direita, $\tilde{R}$

$$
\begin{aligned}
\left(\tilde{U}^{0}\right)^{H} \tilde{U}^{0} & =\left(\tilde{L} D \tilde{R}^{H}\right)^{H} \tilde{L} D \tilde{R}^{H} \\
& =\tilde{R} D\left(\tilde{L}^{H} \tilde{L}\right) D \tilde{R}^{H} \\
& =\tilde{R} D^{2} \tilde{R}^{H}
\end{aligned}
$$

As Equações 2.118 e 2.121 são decomposições espectrais das matrizes $\tilde{U}^{0}\left(\tilde{U}^{0}\right)^{H}$ e $\left(\tilde{U}^{0}\right)^{H} \tilde{U}^{0}$, respectivamente. Os autovalores destas matrizes estão contidos na diagonal principal da matriz $D^{2}$. Embora esta conexão entre a fatoração de $\tilde{U}^{0}$ em SVD e a decomposição espectral das matrizes $\tilde{U}^{0}\left(\tilde{U}^{0}\right)^{H}$ e $\left(\tilde{U}^{0}\right)^{H} \tilde{U}^{0}$ seja natural, na prática, este não é o caminho utilizado no cálculo das matrizes $\tilde{L}, D$ e $\tilde{R}$, ainda que esta abordagem forneça corretamente os resultados. Diversos trabalhos na literatura propõem soluções eficientes para o problema da fatoração 
SVD. (42-47) Este assunto é bem discutido no capítulo 45 do livro "Handbook of Linear Algebra". (48)

Aplicando a fatoração da matriz $\tilde{U}^{0}$ em valores singulares (Equação 2.105) no PAG descrito pela Equação 2.66

$$
\begin{aligned}
\tilde{U}^{1} \tilde{B}_{k} & =\tilde{\mu}_{k} \tilde{U}^{0} \tilde{B}_{k} \\
& =\tilde{\mu}_{k} \tilde{L} D \tilde{R}^{H} \tilde{B}_{k} \\
& =\tilde{\mu}_{k} \tilde{L} D^{\frac{1}{2}}\left(D^{\frac{1}{2}} \tilde{R}^{H} \tilde{B}_{k}\right)
\end{aligned}
$$

Seja o vetor coluna $\tilde{F}_{k}$ definido por

$$
\tilde{F}_{k} \equiv D^{\frac{1}{2}} \tilde{R}^{H} \tilde{B}_{k}
$$

e a identidade

$$
\mathbf{I}=\left(\tilde{R}^{H}\right)^{-1} D^{-\frac{1}{2}} D^{\frac{1}{2}} \tilde{R}^{H}=\tilde{R} D^{-\frac{1}{2}} D^{\frac{1}{2}} \tilde{R}^{H}
$$

então

$$
\begin{aligned}
\tilde{U}^{1} \tilde{B}_{k} & =\tilde{\mu}_{k} \tilde{L} D^{\frac{1}{2}} \tilde{F}_{k} \\
\tilde{U}^{1} \mathbf{I} \tilde{B}_{k} & =\tilde{\mu}_{k} \tilde{L} D^{\frac{1}{2}} \tilde{F}_{k} \\
\tilde{U}^{1}\left(\tilde{R} D^{-\frac{1}{2}} D^{\frac{1}{2}} \tilde{R}^{H}\right) \tilde{B}_{k} & =\tilde{\mu}_{k} \tilde{L} D^{\frac{1}{2}} \tilde{F}_{k} \\
\tilde{U}^{1} \tilde{R} D^{-\frac{1}{2}}\left(D^{\frac{1}{2}} \tilde{R}^{H} \tilde{B}_{k}\right) & =\tilde{\mu}_{k} \tilde{L} D^{\frac{1}{2}} \tilde{F}_{k} \\
\tilde{U}^{1} \tilde{R} D^{-\frac{1}{2}} \tilde{F}_{k} & =\tilde{\mu}_{k} \tilde{L} D^{\frac{1}{2}} \tilde{F}_{k}
\end{aligned}
$$

Multiplicando por $D^{-\frac{1}{2}} \tilde{L}^{-1}$ à esquerda ambos os membros

$$
\begin{aligned}
\left(D^{-\frac{1}{2}} \tilde{L}^{-1}\right) \tilde{U}^{1} \tilde{R} D^{-\frac{1}{2}} \tilde{F}_{k} & =\left(D^{-\frac{1}{2}} \tilde{L}^{-1}\right) \tilde{\mu}_{k} \tilde{L} D^{\frac{1}{2}} \tilde{F}_{k} \\
D^{-\frac{1}{2}} \tilde{L}^{-1} \tilde{U}^{1} \tilde{R} D^{-\frac{1}{2}} \tilde{F}_{k} & =\tilde{\mu}_{k}\left(D^{-\frac{1}{2}} \tilde{L}^{-1} \tilde{L} D^{\frac{1}{2}}\right) \tilde{F}_{k} \\
\left(D^{-\frac{1}{2}} \tilde{L}^{-1} \tilde{U}^{1} \tilde{R} D^{-\frac{1}{2}}\right) \tilde{F}_{k} & =\tilde{\mu}_{k} \tilde{F}_{k} \\
\tilde{G} \tilde{F}_{k} & =\tilde{\mu}_{k} \tilde{F}_{k}
\end{aligned}
$$

onde

$$
\tilde{G} \equiv D^{-\frac{1}{2}} \tilde{L}^{-1} \tilde{U}^{1} \tilde{R} D^{-\frac{1}{2}}
$$

Deste modo, se o problema ordinário de autovalores (Equação 2.135) for resolvido, os autovalores generalizados do PAG (Equação 2.66) são automaticamente obtidos e os autovetores generalizados podem ser calculados através da inversão da Equação 2.125. 
O desenvolvimento apresentado permite a construção dos seguintes passos para a solução da Equação 2.66 utilizando decomposição em valores singulares:

1. A fatoração SVD da matriz $\tilde{U}^{0}$ é computada (Equação 2.105);

2. A matriz $\tilde{G}$ é calculada a partir da Equação 2.136;

3. $\tilde{F}_{k}$ e $\tilde{\mu}_{k}$ são obtidos a partir da solução do problema de autovalores ordinário para $\tilde{G}$ (Equação 2.135);

4. A partir da inversão da Equação $2.125, \tilde{B}_{k}$ é calculado

$$
\tilde{B}_{k}=\tilde{R} D^{-1 / 2} \tilde{F}_{k}
$$

5. A normalização de $\tilde{B}_{k}$ é aplicada (Equação 2.67)

$$
\tilde{B}_{k} \rightarrow \frac{\tilde{B}_{k}}{\sqrt{\tilde{B}_{k}^{T} \tilde{U}^{0} \tilde{B}_{k}}}
$$

\subsubsection{Outros métodos para solução do PAG}

Através de um procedimento semelhante ao que foi discutido na seção 2.4.2, a decomposição espectral da matriz $\tilde{U}^{0}$ poderia ser aplicada ao invés da fatoração SVD. De fato, esta foi a abordagem seguida por Wall e Neuhauser no trabalho original sobre a Diagonalização Filtrada. (22) O desenvolvimento do KBDM utilizando o método da decomposição espectral é detalhado e comparado ao KBDM através do SVD em (23).

Além da transformação em um problema de autovalores ordinário por meio de fatoração, métodos iterativos podem ser utilizados na solução direta do PAG. O algoritmo QZ pertence a esta família de métodos e pode ser interpretado como uma generalização do método QR, utilizado na solução do problema de autovalor ordinário. (49) Este método também é discutido em detalhes no capítulo 6 da referência (50). 


\subsection{Implementação numérica}

\subsubsection{Sinal simulado}

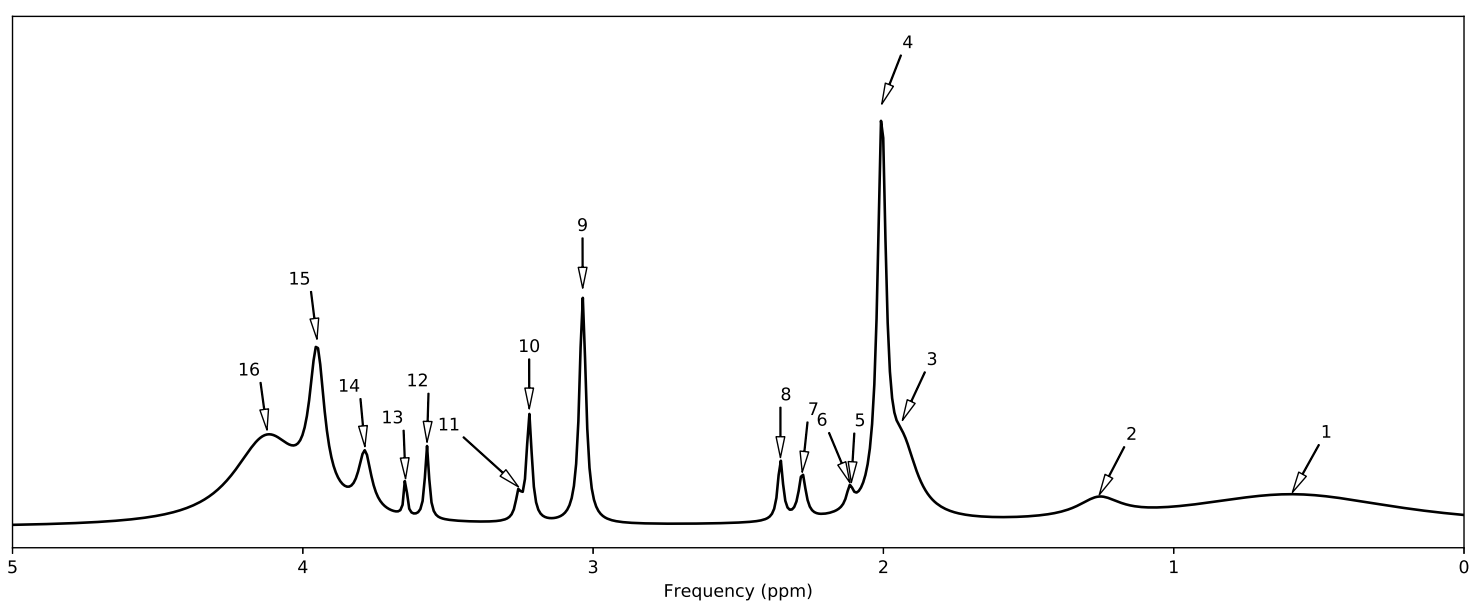

Figura 2.1 - Espectro obtido a partir da Transformada de Fourier Discreta do sinal descrito pela Tabela 2.1 que simula uma aquisição no cérebro em equipamento ressonância magnética de $3 T$. A escala vertical se dá em unidade arbitrária. O eixo horizontal representa o deslocamento químico de cada ressonância em relação à frequência do tetrametilsilano.

Fonte: Elaborada pelo autor.

Através da técnica de fatoração em valores singulares descrita na seção 2.4.2, o algoritmo do KBDM foi desenvolvido em linguagem Python 3.7. Nesta seção, porém, serão enfatizadas apenas discussões e interpretações sobre o método em si e os detalhes da implementação são deixados para o Capítulo 4.

Para compreender o potencial do uso do KBDM, como etapa preliminar, é importante observar o comportamento desta técnica em sinais simulados que representam aquisições ideais. Isto é, desprezando-se as imperfeições causadas por inomogeneidade de campo magnético ou qualquer outro artefato que impeça a representação exata do sinal através da equação 2.1. 0 mesmo sinal simulado utilizado em (28) será utilizado. Este sinal representa uma aquisição na região do cérebro utilizando um equipamento de $3 \mathrm{~T}$, com $N=2048$ pontos e banda espectral de $2 \mathrm{kHz}$.

A Tabela 2.1 contém os parâmetros para construção de cada componente do sinal apresentado na Figura 2.1 através da equação 2.1. Todos os componentes possuem defasagem temporal nula. O número total de pontos complexos do sinal $\tilde{C}$ é de $N=2048$ e o tempo entre duas amostragens consecutivas é de $\tau=0.5 \mathrm{~ms}$. 

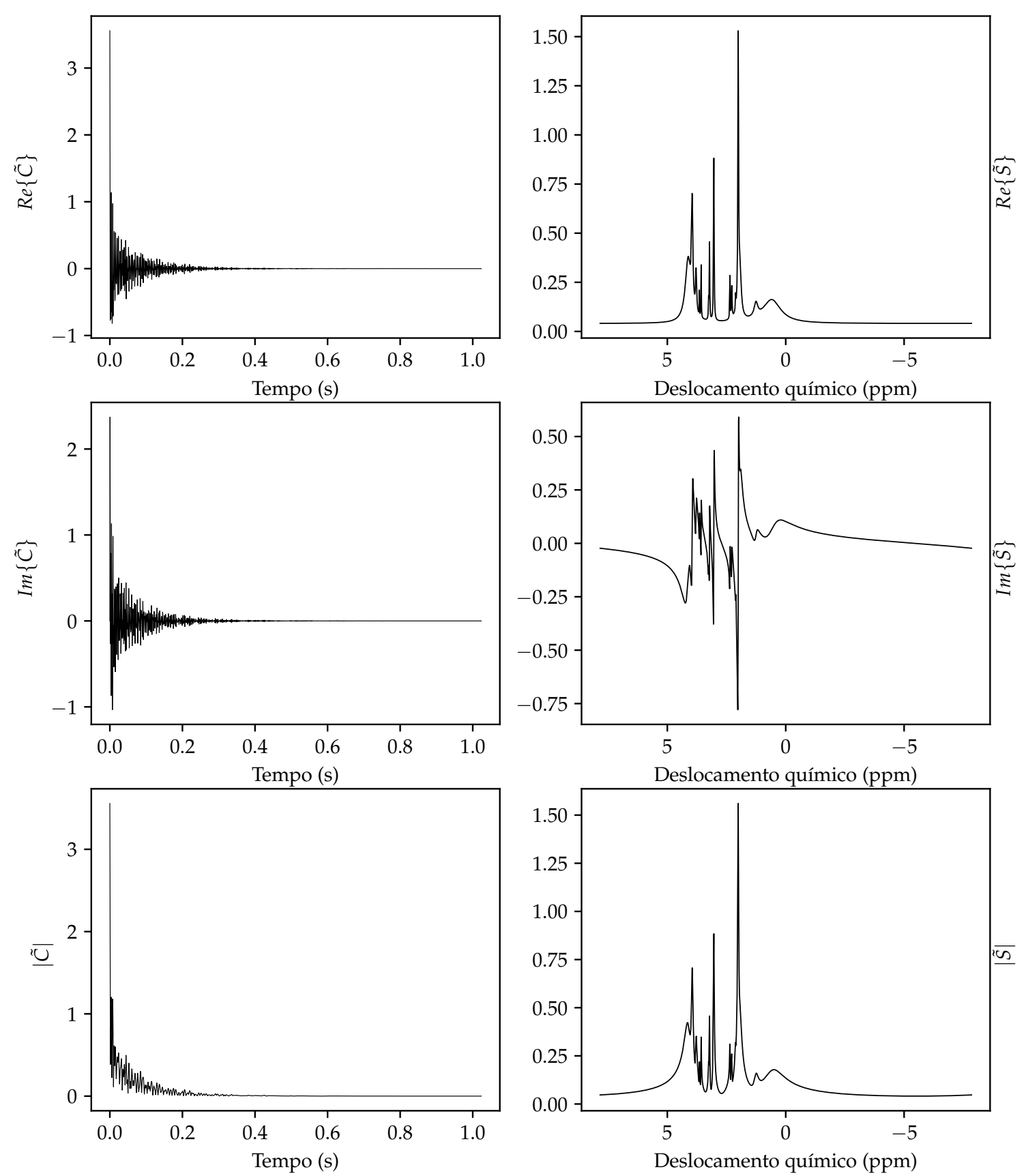

Figura 2.2 - As componentes reais, imaginárias e magnitudes (de cima para baixo) dos sinais nos domínios do tempo, $\tilde{C}$, à esquerda e da frequência, $\tilde{S}$, à direita. $\tilde{C}$ e $\tilde{S}$ possuem $N=2048$ pontos e o intervalo de amostragem é de $\tau=0.5 \mathrm{~ms}$, resultando na largura de banda de $2 \mathrm{kHz}$.

Fonte: Elaborada pelo autor. 
Tabela 2.1 - Amplitude no domínio do tempo, taxa de relaxação transversal $\left(\gamma^{-1}\right)$, frequência em Hertz $(f)$ e deslocamento químico em ppm $(\delta)$ para cada componente $(K=16$ ao total) do sinal gerado a partir do modelo dado pela Equação 2.1. As fases de todos os componentes são nulas $\left(\varphi_{k}=0\right)$.

\begin{tabular}{rrrrrr}
$k$ & Amplitude & $\gamma^{-1}(\mathrm{~s})$ & $f(\mathrm{~Hz})$ & $\delta(\mathrm{ppm})$ & Metabólitos \\
\hline 1 & 1.0000 & 0.0027 & 75.3170 & 0.5912 & Pico 1 \\
2 & 0.1161 & 0.0139 & 160.0646 & 1.2564 & Pico 2 \\
3 & 0.2917 & 0.0199 & 246.4690 & 1.9346 & GABA \\
4 & 0.4289 & 0.0735 & 255.5172 & 2.0056 & NAA \\
5 & 0.0290 & 0.0066 & 268.8984 & 2.1107 & NAAG \\
6 & 0.0184 & 0.0909 & 269.5356 & 2.1157 & Glx2 \\
7 & 0.0451 & 0.0833 & 290.4358 & 2.2797 & GABA2 \\
8 & 0.0427 & 0.1163 & 299.9938 & 2.3547 & Glu \\
9 & 0.2026 & 0.0926 & 386.7804 & 3.0360 & Cr \\
10 & 0.0778 & 0.1136 & 410.2294 & 3.2200 & Cho \\
11 & 0.0202 & 0.1053 & 414.9446 & 3.2570 & M-Ins3 \\
12 & 0.0411 & 0.1471 & 455.0882 & 3.5721 & M-Ins \\
13 & 0.0150 & 0.2222 & 464.5188 & 3.6461 & M-Ins2 \\
14 & 0.1054 & 0.0457 & 482.3604 & 3.7862 & Glx \\
15 & 0.2991 & 0.0400 & 503.3880 & 3.9512 & Cr2 \\
16 & 0.8244 & 0.0088 & 525.3077 & 4.1233 & Cho+M-Ins \\
\hline & & Fonte: Adaptado de $(28)$. &
\end{tabular}

A Figura 2.2 mostra as componentes reais, imaginárias e magnitudes dos sinais do domínio do tempo $(\tilde{C})$ e da frequência $(\tilde{S})$. O espectro $\tilde{S}$ foi calculado a partir da Transformada Rápida de Fourier do sinal $\tilde{C}$. Os detalhes da Transformada de Fourier não serão discutidos neste trabalho e, portanto, sugerimos a referência (6) como consulta.

\subsubsection{Análise de sensibilidade dos parâmetros do KBDM}

Ao longo das seções 2.3 e 2.4, três parâmetros para o KBDM foram definidos: $p, M$ e $J$. O primeiro, definido arbitrariamente com valor unitário $(p=1)$, se refere ao deslocamento relativo entre as matrizes $\tilde{U}^{0}$ e $\tilde{U}^{p}$ e quando $p$ aumenta, menos pontos do início do sinal são considerados na construção de $\tilde{U}^{p}$, muito embora $\tilde{U}^{0}$ ainda retenha informação desta 
mesma região. Deste modo, a escolha $p=1$ parece fisicamente razoável e, portanto, não será realizada nenhuma discussão adicional sobre o assunto nesta seção. Os dois últimos parâmetros, $M$ e $J$, no entanto, possuem papel fundamental na aplicação do método.

O parâmetro $M$ define a dimensionalidade das matrizes $\tilde{U}^{0}$ e $\tilde{U}^{1}$ e a decisão do seu valor possui efeito dual no problema: a definição do truncamento do sinal utilizado para estimativa da lista de parâmetros das componentes; e a determinação do tempo computacional. De acordo com a equação 2.63 , o último elemento da diagonal principal da matriz $\tilde{U}^{1}$ é dado por

$$
\tilde{U}_{M-1, M-1}^{1}=\tilde{C}_{2 M-1}
$$

Ou seja, se $\tilde{C}$ é amostrado com $N$ pontos complexos, o limite superior para $M$ é dado por

$$
M=\frac{N}{2}
$$

Ao computar o KBDM utilizando este limite, todos os pontos do sinal são considerados. Quando o sinal adquirido é "truncado", isto é, quando o tempo de aquisição é inferior ao tempo total necessário para o retorno do sistema de spins à condição de equilíbrio por meio dos mecanismos de relaxação, pode ser vantajoso utilizar todos os pontos do sinal. Entretanto, quando a aquisição é "completa", ou seja, quando o tempo de leitura do sinal é suficiente para a relaxação do sistema, utilizar mais pontos do que o necessário pode aumentar a contribuição do ruído na construção das matrizes $\tilde{U}^{0}$ e $\tilde{U}^{1}$. Além disso, o KBDM possui complexidade computacional da ordem de pelo menos $\mathcal{O}\left(M^{3}\right)$, o que significa que o tempo computacional necessário para a execução do algoritmo aumenta aproximadamente com o cubo de $M$.

Quando a fatoração em valores singulares é adotada na solução da Equação 2.66, a ocorrência do parâmetro $J$ aparece na definição das dimensionalidades das matrizes $\tilde{L}, D$ e $\tilde{R}$. Seu maior significado, entretanto, está associado à determinação do número de valores singulares que serão efetivamente utilizados após a fatoração da matriz $\tilde{U}^{0}$. A matriz $\tilde{G}$ (Equação 2.136) possui dimensionalidade $J \times J$ e, por sua vez, o número de autovalores e autovetores $\tilde{\mu}_{k}$ e $\tilde{F}_{k}$ do problema de autovalores ordinário dado pela Equação 2.135 será $J$. Sendo assim, $J$ também será o número de componentes estimados pelo KBDM utilizando esta abordagem. É importante ressaltar que a fatoração SVD original da matriz $\tilde{U}^{0}$ é computada com $J=M$ e a redução da dimensionalidade das matrizes $\tilde{L}, D$ e $\tilde{R}$ com $J<M$ é realizada posteriormente através do truncamento das mesmas. Consequentemente, o ganho computacional da redução de dimensionalidade é obtido apenas durante a solução do problema de autovalores ordinário (Equação 2.135). 
A Figura 2.3 mostra o erro absoluto médio (EAM) do sinal estimado pelo KBDM em relação ao sinal sintético ideal apresentado na seção 2.5.1 em função dos parâmetros $M$ e $J$. Esta medida de erro pode ser calculada a partir da expressão

$$
\mathrm{EAM}=\frac{1}{N} \sum_{n=0}^{N-1}\left|\tilde{C}_{n}^{e s t}-\tilde{C}_{n}\right|
$$

onde $\tilde{C}_{n}$ e $\tilde{C}_{n}^{e s t}$ são calculados a partir da Equação 2.1 utilizando, respectivamente, os parâmetros da Tabela 2.1 e as estimativas fornecidas pelo KBDM.

Para os valores $J=20,40,60,80,100,150,200,250$ e 300 , o comportamento do erro depende somente dos valores de $M$. É importante notar que $K=16 \mathrm{e}$, portanto, neste exemplo, $J>K$ para todos os valores de $J$. A Figura 2.4 mostra o impacto esperado do parâmetro $J$ quando este é menor do que $K$, uma vez que o número de componentes estimados neste caso é necessariamente inferior ao número de componentes contidos no sinal de entrada. A determinação "incorreta" de $J$ só possui impacto negativo significativo na determinação dos parâmetros dos componentes quando $J<K$. Quando $J>K$, a implementação utilizada da decomposição em SVD contorna internamente o problema da singularidade da matriz $\tilde{U}^{0}$. A Figura 2.5 mostra este comportamento do ponto de vista dos espectros obtidos.

No exemplo mostrado na seção 2.4 , onde a matriz $\tilde{U}^{0}$ possuía dimensionalidade $2 \times 2 \mathrm{e}$ o número de componentes era $K=2$, foi possível obter a solução analítica do problema. Ou seja, as componentes deste exemplo poderiam ser computadas com precisão arbitrária para $M=K=2$. Para a implementação numérica do KBDM aplicado ao sinal simulado com 16 componentes, entretanto, é necessário que $M$ seja maior do que aproximadamente 100 (com $J>K$ ) para que a solução atinja a precisão limitada apenas pelo menor número representável em ponto flutuante (épsilon de máquina) na arquitetura utilizada.

Existe uma diferença fundamental entre as abordagens numérica e analítica na solução do KBDM. De fato, a existência da fórmula de Bhaskara para polinômios de grau 2 permite a obtenção das soluções da equação secular do exemplo analítico (Equação 2.88) para quaisquer parâmetros dos dois componentes existentes no sinal. Embora muito mais elaborados e numericamente mal comportados por fazerem uso abusivo de radicais, métodos para obter as raízes de polinômios de grau 3 e 4 são também conhecidos. Em teoria, isto viabilizaria a extensão do exemplo analítico para $M=K=3$ e $M=K=4$, por exemplo. Entretanto, o Teoria de Galois afirma que nenhum método direto, fórmula ou sequência de passos finitos pré-determinados poderiam existir no cálculo das raízes de polinômios de grau maior do que 4 e qualquer método para execução de tal procedimento necessariamente será de natureza iterativa. (50-52) É importante ressaltar que essa teoria não prevê a impossibilidade da criação 


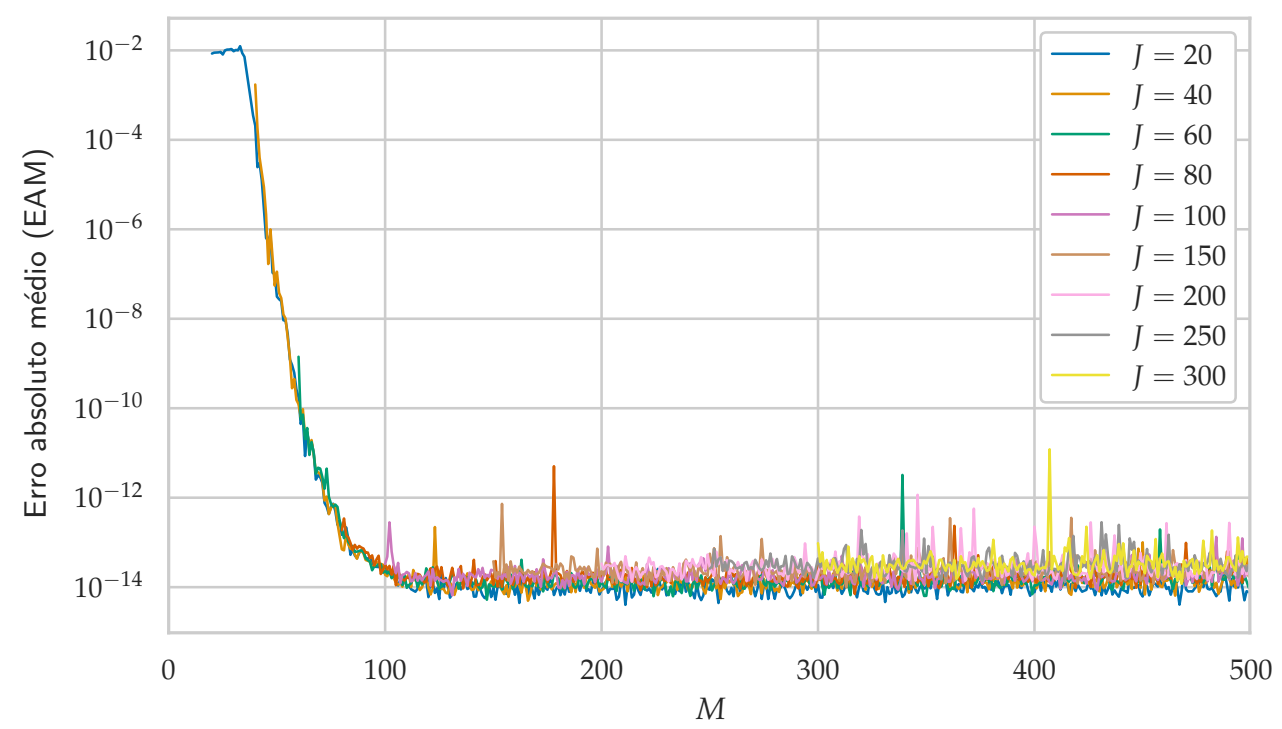

Figura 2.3 - Erro absoluto médio (Equação 2.141), em função do parâmetro $M$ para vários valores de $J>K$ ao utilizar o KBDM no sinal descrito pela Tabela 2.1. A existência de cada curva obedece à condição $M \geq J$, que é uma restrição do KBDM resolvido através da fatoração SVD. Todas as curvas possuem comportamento semelhante quando $J>$ $K$, indicando baixa sensibilidade ao parâmetro $J$ se esta restrição for obedecida. $\mathrm{O}$ pequeno aumento sistemático e proporcional a $J$ do erro é causado pelo acúmulo das contribuições das componentes espúrias de baixa amplitude que não foram filtradas nesta análise.

Fonte: Elaborada pelo autor.

de fórmulas ou procedimentos para casos particulares e muito menos determina a inexistência das soluções, mas apenas delimita a natureza dos procedimentos possíveis para o caso geral.

O cálculo de raízes de polinômios e o problema de autovalores possuem conexão intrínseca. De fato, vários pacotes de software utilizam a diagonalização da Matriz Companheira no cálculo das raízes de polinômios gerais. Deste modo, como todo problema de autovalor pode ser reduzido ao problema de se obter raízes da equação secular, que é um polinômio, as consequências da Teoria da Galois se estende ao problema de autovalores. Isto é, para uma matriz diagonalizável $M \times M$ qualquer com $M>4$, apenas métodos iterativos serão capazes de prover soluções dentro de uma dada precisão numérica no caso geral. O núcleo do modelo matemático do KBDM é totalmente dependente do uso de diagonalização de matrizes e, portanto, estará automaticamente sujeito às consequências do uso de métodos iterativos em relação às questões de convergência.

O que será explorado agora é o aumento do número de pontos necessários $(M>K)$ na reconstrução do sinal com precisão numérica máxima em função da característica de seus componentes. Mais precisamente, será investigado o efeito da superposição dos picos no 


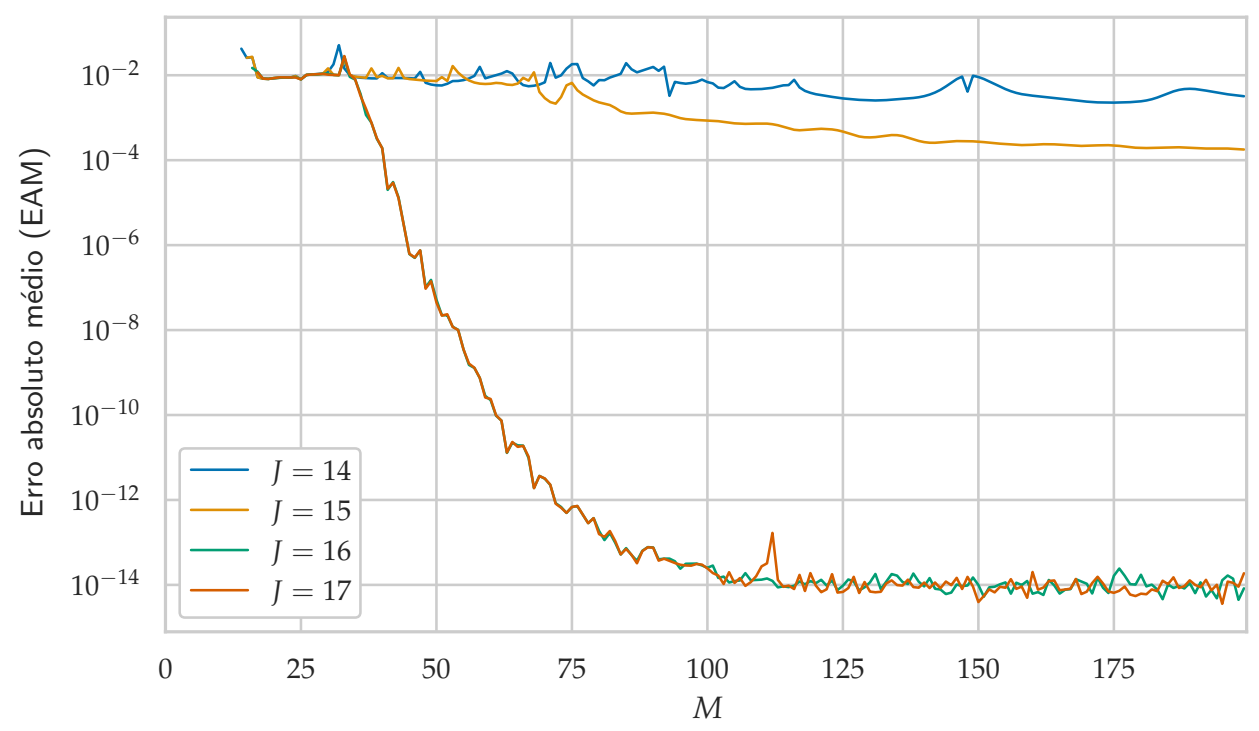

Figura 2.4 - Erro absoluto médio (Equação 2.141) em função do parâmetro $M$ para $J=14,15,16$ e 17 ao utilizar o KBDM no sinal descrito pela Tabela 2.1. O erros relativos elevados para $J=14$ e $J=15$ são esperados, pois neste caso o número de componentes estimados é necessariamente menor do que o número de componentes contidos no sinal. O comportamento das curvas respectivas a $J=16$ e $J=17$ é semelhante, mostrando que, neste caso, a componente espúria adicional estimada possui contribuição resultante desprezível no sinal estimado.

Fonte: Elaborada pelo autor.

KBDM. O alto grau de superposição das linhas espectrais do sinal descrito pela Tabela 2.1 pode ser observado na Figura 2.1. Para entender o problema, pode-se utilizar um sinal composto por um "pente" de $K$ picos igualmente espaçados com taxas de relaxamento e amplitudes idênticas, tal que

$$
\begin{gathered}
\tilde{C}_{n}=\sum_{k=0}^{K-1} a e^{-\gamma n \tau} e^{i \omega_{k} n \tau} \\
\omega_{k}=2 \pi\left(k-\frac{(K-1)}{2}\right) \Delta f
\end{gathered}
$$

onde, por simplicidade, $K$ é um número ímpar.

A Figura 2.6 mostra o espectro gerado a partir do sinal descrito pelas Equações 2.142 e 2.143 e os respectivos valores do erro absoluto médio para $K=9$ e $\Delta f=220 \mathrm{~Hz}$ são mostrados na Figura 2.7. Como não há superposição de picos, o sinal pode ser reconstruído com precisão numérica máxima para $M=K=9$.

Quando o espaçamento espectral entre picos consecutivos $(\Delta f)$ é reduzido, os efeitos da sobreposição começam a surgir. A Figura 2.8 mostra o espectro do sinal quando $K=9 \mathrm{e}$ $\Delta f=50 \mathrm{~Hz}$. Os efeitos causados pela sobreposição neste caso podem ser observados através 

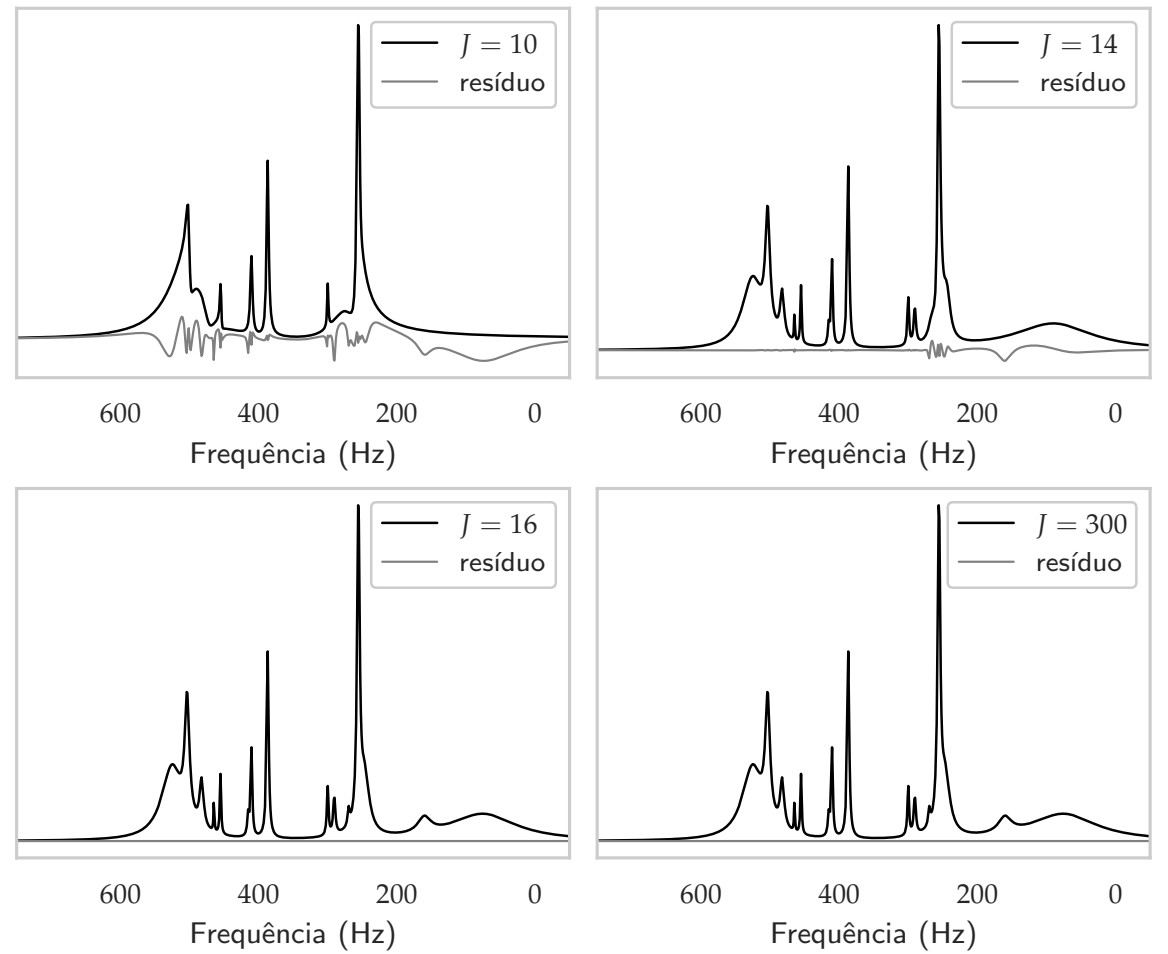

Figura 2.5 - Espectros estimados utilizando o KBDM com $M=300$ para o sinal descrito pela Tabela 2.1 para $J=10,14,16$ e 300 . Para um sinal ideal modelado exatamente pela Equação 2.1, a implementação do KBDM é sensível ao parâmetro $J$ apenas quando $J<M$.

Fonte: Elaborada pelo autor.

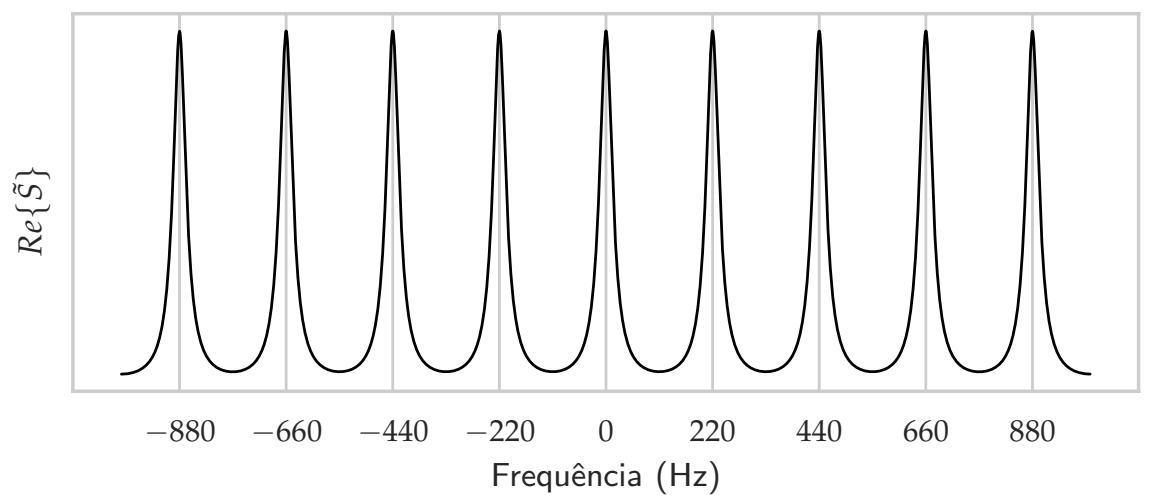

Figura 2.6 - Parte real do espectro computado a partir do sinal gerado pelas Equações 2.142 e 2.143 para $K=9$ e $\Delta f=220 \mathrm{~Hz}$.

Fonte: Elaborada pelo autor. 


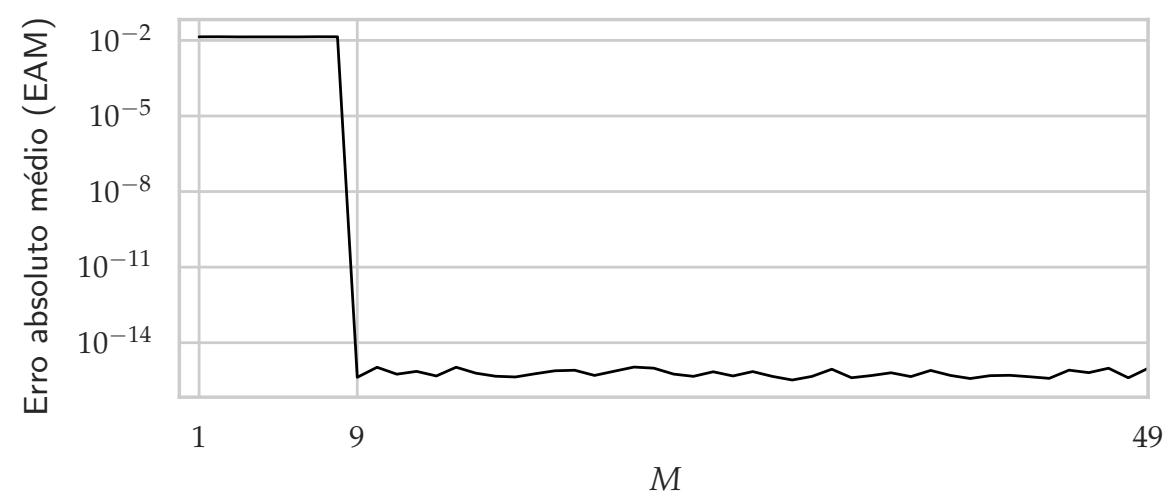

Figura 2.7 - Erro absoluto médio da reconstrução do sinal apresentado na Figura 2.6 para $K=9$ e $\Delta f=220 \mathrm{~Hz}$. Neste caso, $M=K=9$ é suficiente para a reconstrução do sinal com precisão limitada pelo épsilon de máquina.

Fonte: Elaborada pelo autor.

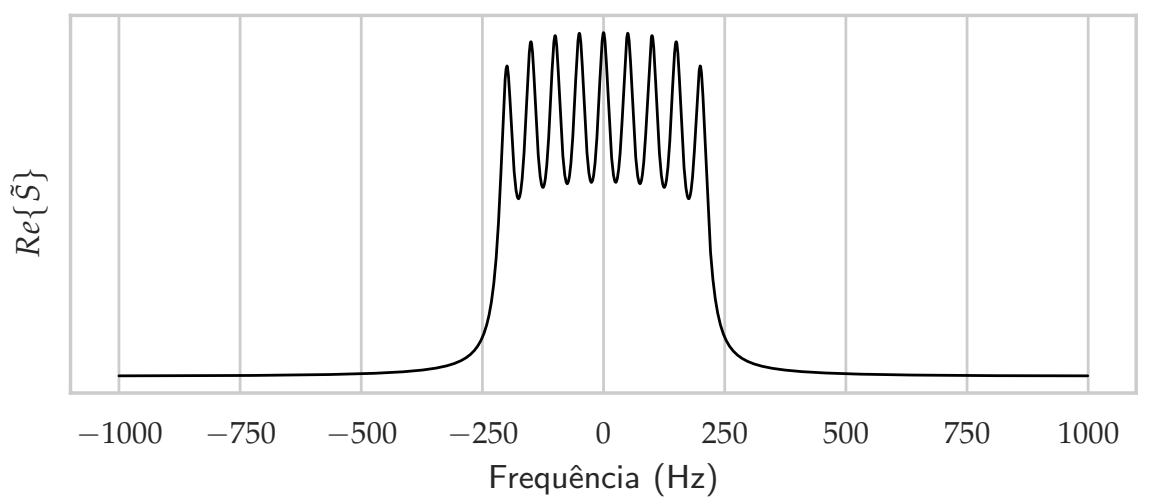

Figura 2.8 - Parte real do espectro computado a partir do sinal gerado pelas Equações 2.142 e 2.143 para $K=9$ e $\Delta f=50 \mathrm{~Hz}$. A superposição dos picos é acentuada neste caso.

Fonte: Elaborada pelo autor. 


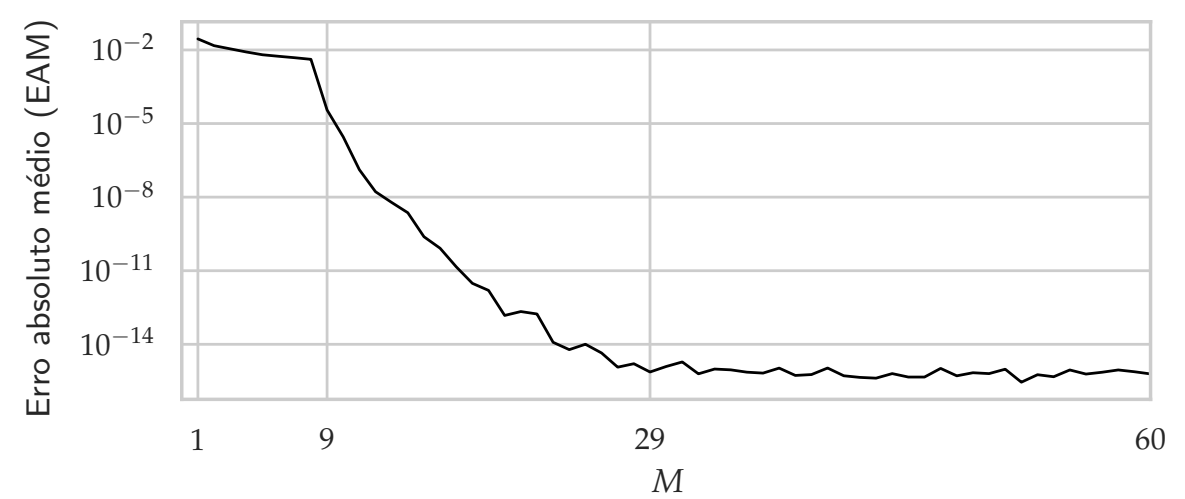

Figura 2.9 - Impacto da sobreposição dos picos no erro absoluto médio durante a reconstrução do sinal apresentado na Figura 2.8. Desta vez, o ajuste realizado através do KBDM atinge precisão numérica máxima apenas quando $M \approx 29$.

Fonte: Elaborada pelo autor.

dos valores de erro absoluto médio apresentados na Figura 2.9.

A Figura 2.10 mostra o efeito da superposição em função de diferentes separações entre picos e também número de picos. A partir desta análise, é possível concluir que quanto maior a quantidade de picos superpostos ou quanto menor for a separação entre os mesmos, maior deverá ser o número de pontos necessários para obtenção de valores ótimos através das estimativas feitas pelo KBDM.

A presença de ruído é uma característica inevitável nos sinais de ressonância magnética. O tipo de ruído que será considerado possui as seguintes características:

- Possui energia uniformemente distribuída em toda a banda espectral;

- Segue a distribuição normal $\mathcal{N}(\mu, \sigma)$. Isto é, a função de densidade de probabilidade que o representa é dada por

$$
f(x ; \mu, \sigma)=\frac{1}{\sqrt{2 \pi \sigma^{2}}} e^{-\frac{(x-\mu)^{2}}{2 \sigma^{2}}}
$$

- Possui média zero $(\mu=0)$ e, portanto, sua distribuição é determinada somente pelo desvio padrão, $\sigma$;

- O ruído presente em cada amostra $n$ do sinal $\tilde{C}_{n}$ não possui dependência com as outras amostras $n^{\prime}$. A partir dessa hipótese é possível afirmar que não existe correlação temporal entre as amostras neste tipo de ruído;

- As contribuições reais e imaginárias não são correlacionadas. Essa hipótese é baseada 


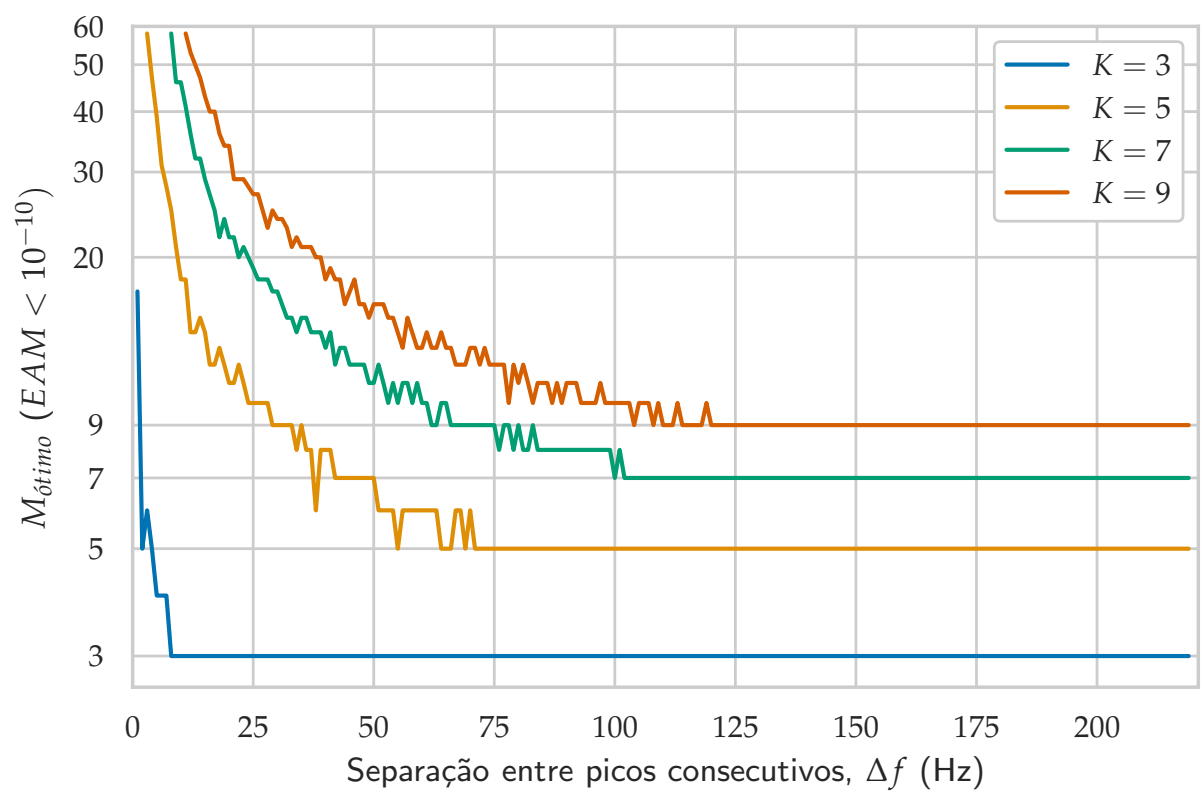

Figura 2.10 - Valor ótimo do parâmetro $M$ do KBDM para o sinal descrito pelas Equações 2.142 e 2.143 em função da separação entre picos consecutivos no domínio

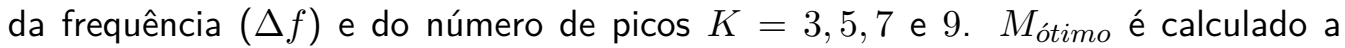
partir do valor mínimo do parâmetro $M$ onde o EAM é inferior a $10^{-10}$. O eixo vertical é apresentado em escala logarítmica.

Fonte: Elaborada pelo autor.

no fato de que as aquisições em fase e em quadratura do sinal através das bobinas receptoras são realizadas de maneira independente.

Esta categoria específica de pertubação no sinal recebe o nome de "ruído gaussiano branco".

O sinal de entrada passa a partir de agora a receber a contribuição do ruído $\tilde{x}_{n}$, tal que

$$
\begin{gathered}
\tilde{C}_{n} \rightarrow \tilde{C}_{n}+\tilde{x}_{n} \\
\tilde{x}_{n}=x_{n}^{\text {real }}+i x_{n}^{i m a g} \\
X^{\text {real }}, X^{i m a g} \sim \mathcal{N}(0, \sigma)
\end{gathered}
$$

onde $X^{\text {real }}$ e $X^{i m a g}$ são as variáveis aleatórias independentes que representam, respectivamente, as contribuições reais e imaginárias do ruído presente no sinal; e $x_{n}^{\text {real }}$ e $x_{n}^{\text {imag }}$ as suas realizações.

Na Figura 2.11 são apresentados os erros absolutos médios na presença de ruído com níveis estabelecidos pelos desvios padrões $\sigma=0.001,0.003,0.01$ e 0.03 para o sinal determinado a partir dos parâmetros da Tabela 2.1 através das estimativas realizadas pelo KBDM. De 


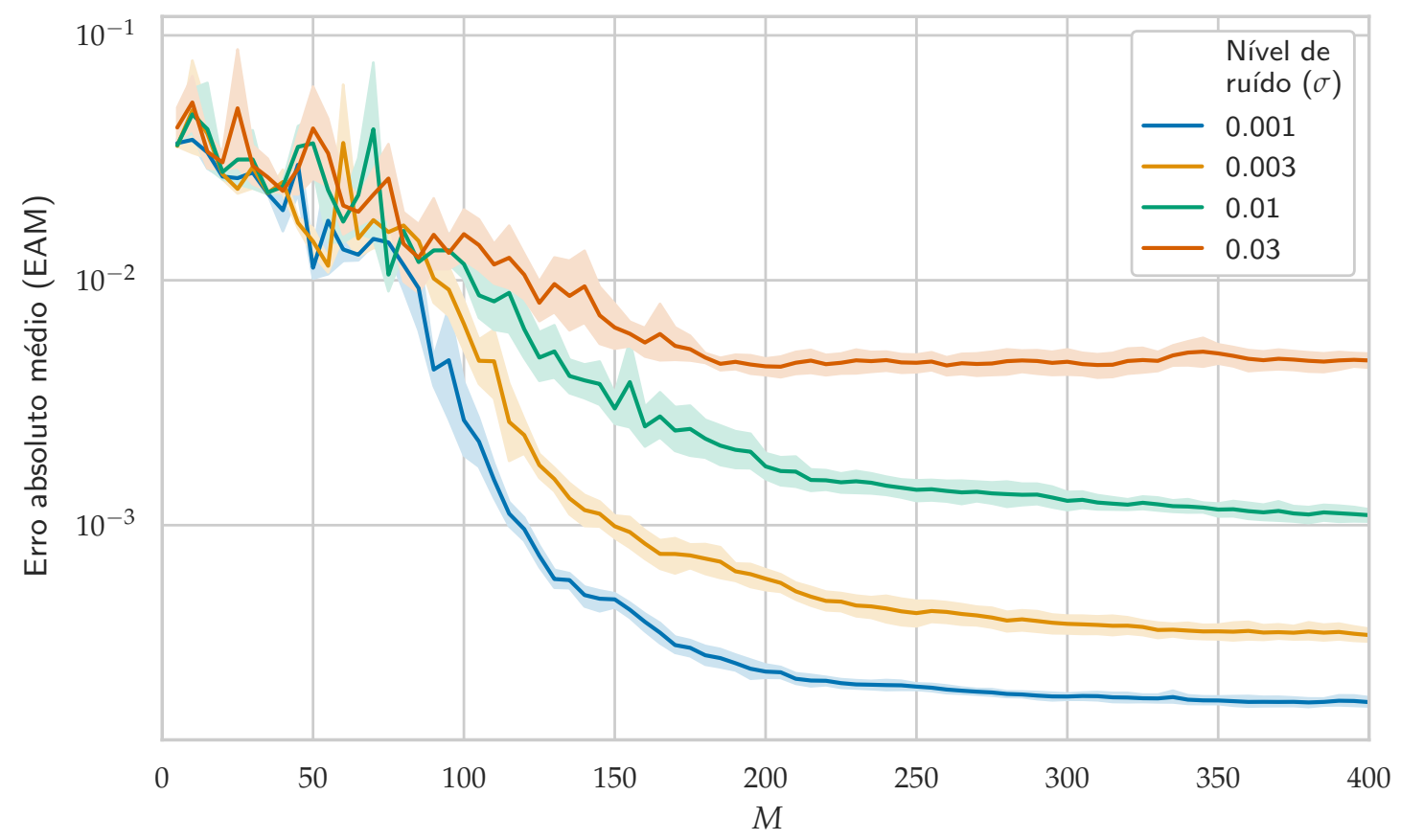

Figura 2.11 - Erros absolutos médios (EAM) na presença de ruído com níveis definidos pelos desvios padrões $\sigma=0.001,0.003,0.01$ e 0.03 para o sinal parametrizado pela Tabela 2.1. Para esta análise, foram realizadas 10 observações para cada valor do parâmetro $M$ com $J=\min (16, M)$ e $M=5,10,15, \ldots, 400$. As linhas escuras representam o valor médio obtido paras os valores observados do EAM e o sombreamento em torno das curvas simbolizam as margens de erro estimadas através dos respectivos desvios padrões.

Fonte: Elaborada pelo autor. 




Figura 2.12 - Erro absoluto médio (EAM) em função do nível de ruído determinado pelo desvio padrão $\sigma$ da distribuição que o descreve para as estimativas calculadas através do KBDM com $M=400$ e $J=16$. Ambos os eixos são apresentados em escala logarítmica.

Fonte: Elaborada pelo autor.

maneira geral, é possível observar que o EAM foi significantemente afetado e o valor $M$ de estabilização possui alta dependência do desvio padrão do ruído $\sigma$.

Assumindo o valor de estabilização do erro das estimativas do $\mathrm{KBDM}$ em $M=400$, a Figura 2.12 mostra de maneira mais abrangente os valores do EAM em função do desvio padrão $\sigma$ da distribuição do ruído.

Exemplos de espectros estimados através do KBDM a partir de sinais ruidosos com $\sigma=$ $0.001,0.003,0.01$ e 0.03 são mostrados na Figura 2.13. A diferença residual entre o espectro estimado e o sinal simulado sem ruído também é apresentada.

Um outro problema prático imposto pela presença de ruido é determinação do valor ótimo para o parâmetro $J$. Quando não há ruído, foi mostrado anteriormente que a única restrição significativa sobre este parâmetro é que $J \geq K$. Neste mesmo cenário, existem muitas ordens de grandeza na diferença entre os casos $J \geq K$ e $J<K$ para o valor do EAM (Figura 2.4). Infelizmente, essa diferença se reduz abruptamente na medida que o nível de ruído aumenta, conforme mostrado na Figura 2.14. Isso gera uma complicação no que diz respeito à detecção automática deste valor para o caso geral, uma vez que, ao contrário dos exemplos sintéticos onde se conhece o número de componentes, em aplicações práticas a informação sobre o valor exato de $K$ é normalmente inexistente.

O problema na determinação de $J$ pode ser observado através da inspeção dos valores 

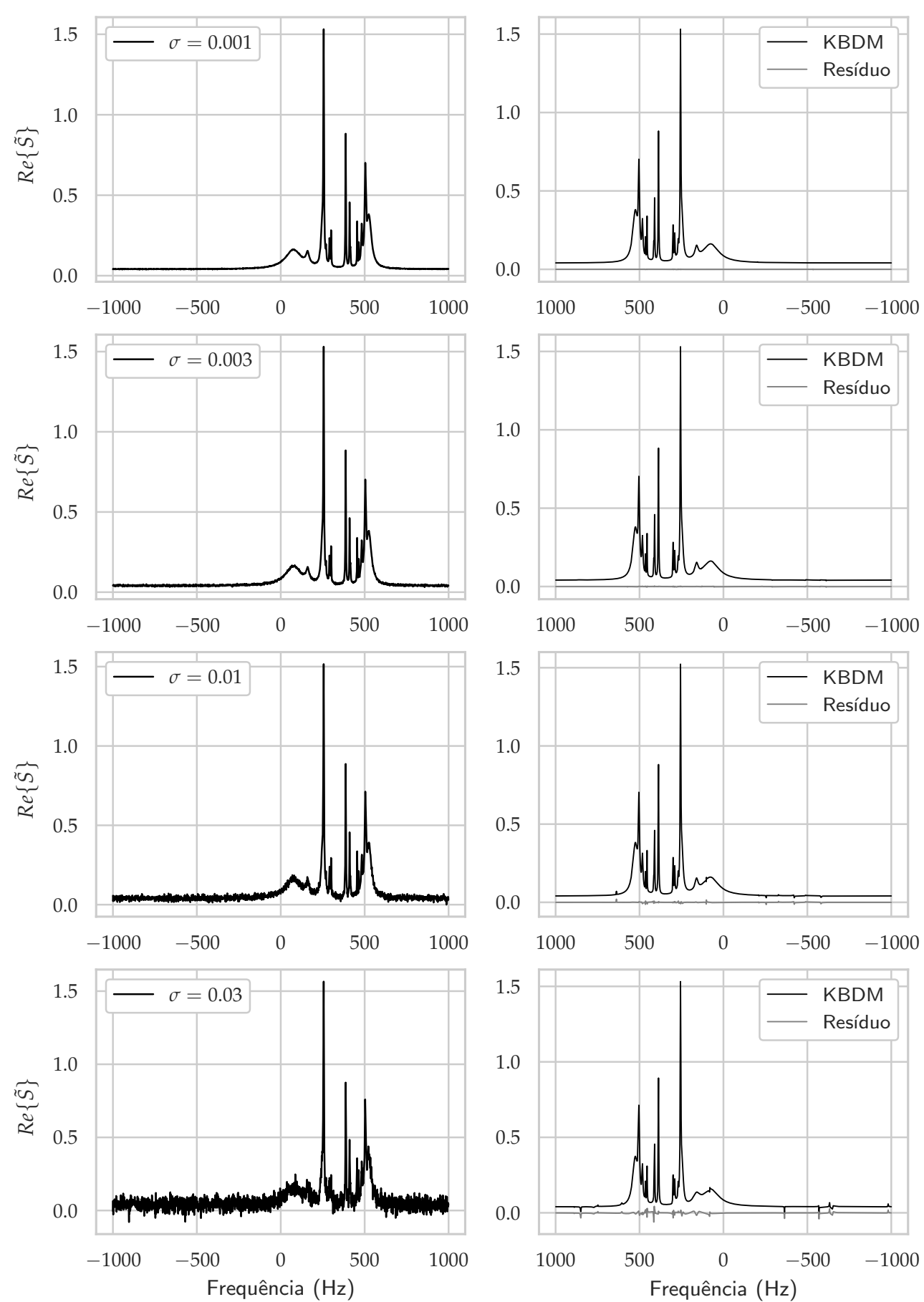

Figura 2.13 - Exemplos de espectros estimados a partir do KBDM para sinais ruidosos. Os níveis de ruído variam do menor para o maior (de cima para baixo) para os valores de desvio padrão $\sigma=0.001,0.003,0.01$ e 0.03 . Do lado direito são apresentados os sinais de entrada e do lado esquerdo as estimativas obtidas através do KBDM (curva preta) e os respectivos resíduos (curva cinza) calculados a partir da diferença entre o espectro obtido e o valor verdadeiro sem a presença de ruído. Em todas as estimativas foram utilizados os parâmetros $M=400$ e $J=16$.

Fonte: Elaborada pelo autor. 


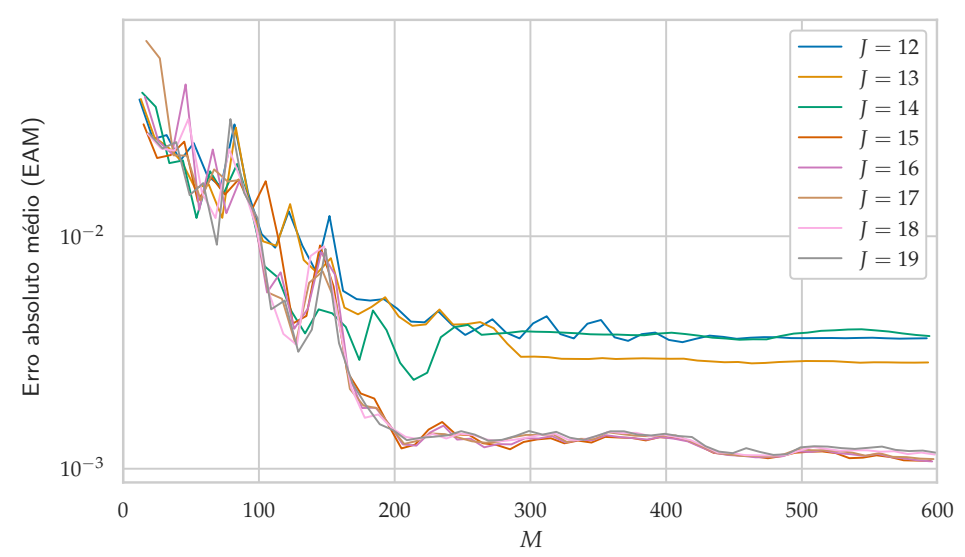

Figura 2.14 - Erro absoluto médio (EAM) das estimativas do KBDM no sinal descrito pela Tabela 2.1 em função do parâmetro $M$ para diferentes valores valores de $J$ na presença de ruído gaussiano com $\sigma=0.01$. Comparado aos valores obtidos para o sinal sem ruído na Figura 2.4, a diferença entre os casos $J \geq K$ e $J<K$ é muito menor para este nível de ruído.

Fonte: Elaborada pelo autor.

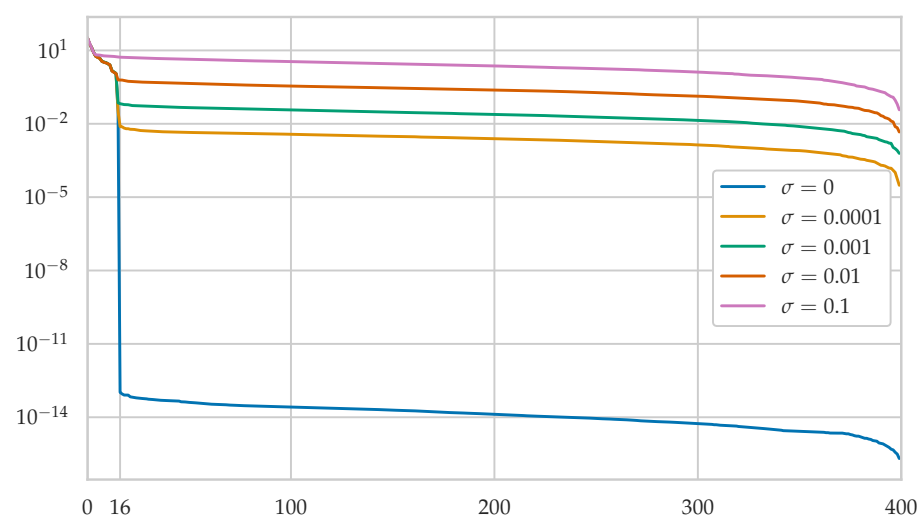

Figura 2.15 - Valores singulares obtidos através da implementação SVD utilizada para a matriz $\tilde{U}^{0}$ em função dos níveis de ruído do sinal.

Fonte: Elaborada pelo autor.

singulares contidos na matriz diagonal $D$ (Equação 2.105). A Figura 2.15 mostra a comparação dos valores singulares obtidos para diferentes níveis de ruído. Na verdade, a fatoração SVD na solução do KBDM possui separabilidade melhor do que ocorreria se a fatoração espectral fosse utilizada, conforme mostrado em (23). Este foi um dos fatores determinantes da escolha do SVD como estratégia para solução do PAG(Equação 2.66). 


\section{Promediação através do agrupamento de linhas espectrais}

\subsection{Introdução}

Neste capítulo será discutido o tema central deste trabalho, que é o uso de agrupamento e promediação das linhas espectrais estimadas através de múltiplas execuções do KBDM no espaço de parâmetros do sinal. Esta técnica foi inspirada em resultados de trabalhos anteriores, onde foi mostrado que a utilização de promediação dos espectros estimados pelo FDM é capaz de reduzir o impacto da presença de ruído. $(21,39-40,53)$ Embora nesses trabalhos esta abordagem tenha permitido a obtenção de espectros de qualidade superior, elas não possibilitaram a obtenção de uma lista compacta de parâmetros resultantes da promediação. (21)

A proposta de um método que cumpre esta tarefa será apresentada. O ponto chave desta abordagem consiste em realizar a amostragem de múltiplas linhas estimadas pelo KBDM utilizando o mesmo espectro, mas explorando a variedade existente nos resultados de cada execução. A amostragem pode ser realizada a partir da variação do parâmetro $M$ ou através da adição de uma pequena porção de ruído no sinal, denominado pseudo-ruído.

A determinação paramétrica de cada componente do sinal possui papel importante na quantificação ou supressão de picos indesejados. Como o FDM e o KBDM foram desenvolvidos para serem utilizados em aplicações fora do escopo da espectroscopia in vivo, não foi encontrado na literatura nenhuma outra abordagem semelhante ou equivalente ao que será apresentado neste capítulo e, portanto, trata-se de uma contribuição original deste trabalho.

Para melhor entendimento do método, o problema foi dividido em partes fundamentais que serão discutidas separadamente. Sendo elas

- Amostragem (Seção 3.2);

- Agrupamento (Seção 3.3); 
- Transformações no espaço de dados (Seção 3.4);

- Ajuste iterativo (Seção 3.5).

\subsection{Amostragem}

O procedimento de amostragem consiste no levantamento de múltiplas estimativas através do KBDM para os parâmetros $\left\{\left(\tilde{a}_{k}, \widetilde{\Omega}_{k}\right)\right\}$ que permitem a reconstrução do sinal a partir da Equação 2.1. O objetivo deste processo é explorar a sensibilidade do algoritmo à presença de ruído através da promediação dos múltiplos resultados obtidos, representados pelas linhas espectrais estimadas, resultando em uma estimativa superior do espectro ou de sua respectiva lista de parâmetros.

A seguir serão discutidas as duas maneiras já apresentadas na literatura para amostragem do sinal: a adição de pseudo-ruído (Seção 3.2.1); e o uso de múltiplos truncamentos (Seção 3.2.2).

\subsubsection{Adição de pseudo-ruído}

Considere um sinal de entrada $\tilde{C}$ representado através de $N$ pontos complexos $\tilde{C}_{n}$. Cada amostra do conjunto de linhas espectrais $\left\{\left(\tilde{a}_{j}^{w}, \widetilde{\Omega}_{j}^{w}\right)\right\}$ com $w=0,1, \ldots, W-1$ gerada utilizando adição de pseudo-ruído é calculada a partir da execução do KBDM no sinal $\tilde{C}^{w}$, onde

$$
\tilde{C}_{n}^{w}=\tilde{C}_{n}+\tilde{x}_{n}^{w}
$$

sendo $\operatorname{Re}\left\{\tilde{x}_{n}^{w}\right\}$ e $\operatorname{Im}\left\{\tilde{x}_{n}^{w}\right\}$ realizações independentes da variável aleatória $X$ que representa o ruído gaussiano branco adicionado, tal que $X \sim \mathcal{N}\left(0, \sigma_{p}\right)$ com

$$
\sigma_{p} \ll \sigma
$$

onde $\sigma$ e $\sigma_{p}$ são, respectivamente, os desvios padrões do ruído presente no sinal de entrada e do pseudo-ruído sintetizado.

Se cada amostra for computada utilizando os parâmetros fixos $M$ e $J(J<M)$ do KBDM, 
o total de $W J$ linhas $\left\{\left(\tilde{a}_{j}^{w}, \widetilde{\Omega}_{j}^{w}\right)\right\}$ serão obtidas. Assumindo $J \geq K$, pelo menos $W(J-K)$ componentes espúrias estarão presentes nas estimativas.

\subsubsection{Múltiplos truncamentos}

Nesta abordagem, ao invés da adição de ruído sintético, a instabilidade das estimativas em função do parâmetro $M$ na presença do ruído do próprio sinal é explorada.

Para um mesmo sinal $\tilde{C}$ de $N$ pontos complexos $\tilde{C}_{n}$, são obtidas $W$ amostras de linhas espectrais estimadas pelo KBDM para $M=M_{w}$ com $M_{w} \leq N / 2$, onde $w=0,1, \ldots, W-1$. Se fixado o parâmetro $J<M$ o número total de linhas espectrais $\left\{\left(\tilde{a}_{j}^{w}, \widetilde{\Omega}_{j}^{w}\right)\right\}$ será novamente $W J$. O número de mínimo de componentes espúrias também será o mesmo obtido através da adição de pseudo-ruído nestas condições.

A construção das matrizes $\tilde{U}^{0}$ e $\tilde{U}^{1}$ para cada amostra $w$ é calculada a partir de apenas $N_{w}=2 M_{w}$ pontos complexos do sinal, justificando o nome dado a este procedimento.

\subsubsection{Aspectos da amostragem nas diferentes abordagens}

No caso da adição de pseudo-ruído, quando $\sigma_{p}$ é muito pequeno, a variabilidade das estimativas pode não ser suficiente. Por outro lado, quando $\sigma_{p}$ é relativamente grande, algumas componentes do sinal podem deixar de ser detectadas pelo KBDM. Desta forma, o balanço adequado para o parâmetro $\sigma_{p}$ deve ser encontrado.

Do ponto de vista da amostragem por múltiplos truncamentos, um fator determinante se encontra na definição do intervalo de valores para o parâmetro $M$, sendo importante considerar a análise feita na seção 2.5.2. Quando $M_{w}$ é relativamente pequeno, as estimativas se tornam imprecisas. Quando $M_{w}$ aumenta o tempo de execução cresce e a quantidade proporcional de ruído em cada ponto do sinal também aumenta. 


\subsubsection{Promediação no domínio temporal ou espectral}

A promediação no domínio temporal a partir de $W$ amostras dos resultados do KBDM utilizando adição de pseudo-ruído ou através de múltiplos truncamentos pode ser expressa a partir do cálculo de cada ponto do sinal resultante

$$
\tilde{C}_{n}^{\prime}=\frac{1}{W} \sum_{w=0}^{W-1} \tilde{C}_{n}^{w}
$$

Este procedimento pode ser realizado de maneira análoga no domínio espectral

$$
\tilde{S}_{n}^{\prime}=\frac{1}{W} \sum_{w=0}^{W-1} \tilde{S}_{n}^{w}
$$

onde $\tilde{S}^{w}$ é calculado através da transformada de Fourier do sinal $\tilde{C}^{w}$.

Embora o sinal resultante $\tilde{C}_{n}^{\prime}$ ou o espectro $\tilde{S}_{n}^{\prime}$ sejam provavelmente de qualidade superior ao de cada amostra individual $w$, uma lista de parâmetros que sumarizam as componentes estimadas a partir das $W$ amostras não pode ser obtida diretamente através desta técnica.

\subsubsection{Promediação no espaço de parâmetros}

Para um dado componente genuíno do sinal de entrada, $c_{k}=\tilde{a}_{k} e^{i \widetilde{\Omega}_{k} \tau}$, será considerada uma estimativa bem sucedida através da $w$-ésima amostra do KBDM por qualquer um dos métodos discutidos da seção 3.2 se, entre os componentes gerados a partir das linhas obtidas, estiver presente o componente $c_{k}^{w}=\tilde{a}_{k}^{w} e^{i \widetilde{\Omega}_{k}^{w} \tau}$, tal que

$$
\theta\left(c_{k}, c_{k}^{w}\right)>\theta_{0}
$$

onde $\theta$ é uma medida de similaridade entre dois componentes individuais e $\theta_{0}$ é o valor mínimo aceitável deste critério.

A principal hipótese do método proposto pode ser enunciada da seguinte maneira: para cada componente genuíno $k$ de um sinal que contém ruído (Equação 2.145), a média dos parâmetros dos componentes estimados que satisfazem ao respectivo critério de similaridade (Equação 3.5) fornece uma estimativa superior, $c_{k}^{\prime}$, tal que

$$
c_{k}^{\prime}=\tilde{a}_{k}^{\prime} e^{i{\widetilde{\Omega^{\prime}}}_{k} \tau}
$$


onde

$$
\begin{aligned}
& \tilde{a}_{k}^{\prime}=\frac{1}{W^{\prime}} \sum_{w^{\prime}=0}^{W^{\prime}-1} \tilde{a}_{k}^{w^{\prime}} \\
& \tilde{\Omega}_{k}^{\prime}=\frac{1}{W^{\prime}} \sum_{w^{\prime}=0}^{W^{\prime}-1} \tilde{\Omega}_{k}^{w^{\prime}}
\end{aligned}
$$

e

$$
\begin{gathered}
c_{k}^{w^{\prime}}=\tilde{a}_{k}^{w^{\prime}} e^{i \widetilde{\Omega}_{k}^{w^{\prime}} \tau} \\
\theta\left(c_{k}, c_{k}^{w^{\prime}}\right)>\theta_{0} \\
W^{\prime} \leq W
\end{gathered}
$$

Portanto, a promediação ocorre no espaço de parâmetros e a lista de linhas resultantes $\left\{\left(\tilde{a}_{k}^{\prime}, \tilde{\Omega}_{k}^{\prime}\right)\right\}$ são utilizadas na reconstrução do sinal. Esta hipótese é fundamentada na constatação empírica que pode ser observada a partir das Figuras 3.1-3.9. O desvio padrão do ruído utilizado nesta análise é dado por $\sigma=0.004$. Apenas os picos que possuem demasiado grau de superposição tiveram sua reconstrução comprometida (picos \#5 e \#6). Enquanto o pico \#6 apresenta erros na amplitude e frequência significativamente maiores do que os demais, o pico \#5 não pôde ser reconstruído de acordo com o critério utilizado na identificação das linhas obtidas.

Este mesmo comportamento pode ser analisado através da Figura 3.10, onde desta vez as taxas médias (barras verticais cinzas) e os respectivos desvios padrões (barras verticais vermelhas) das estimativas bem sucedidas para cada pico são mostradas em função dos níveis de ruído $(\sigma=0.01,0.02,0.03,0.04)$. Novamente, o pico \#5 não foi reconstruído em nenhum caso. Além disso, os picos \#2, \#6, \#11 e \#13 possuem a maior sensibilidade ao ruído. 


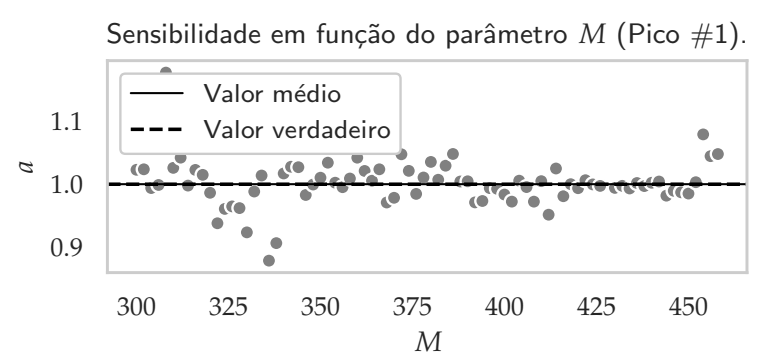

Distribuição. Erro relativo: $0.006 \%$ (Pico \#1).

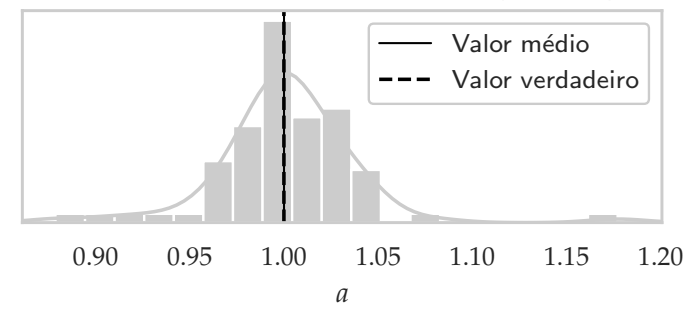

Sensibilidade em função do parâmetro $M$ (Pico \#2).

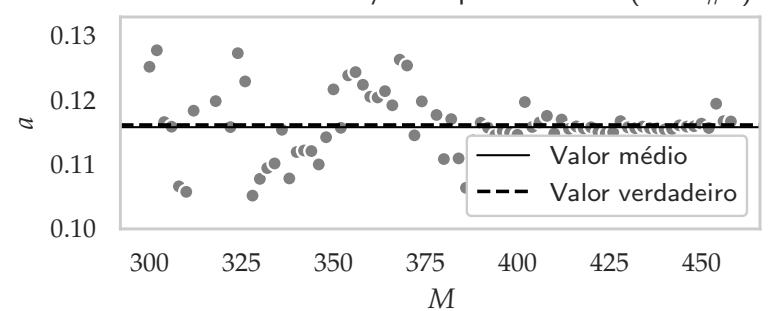

Distribuição. Erro relativo: $0.299 \%$ (Pico \#2).

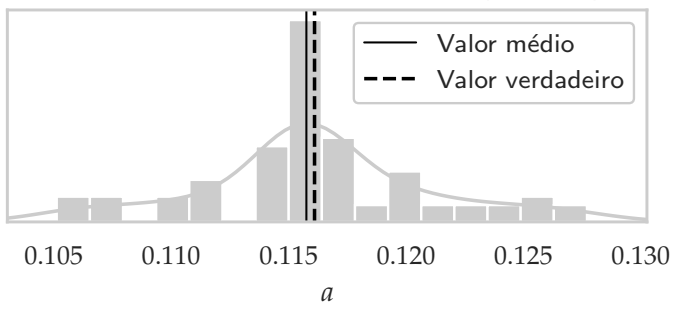

Distribuição. Erro relativo: 1.477\% (Pico \#3).
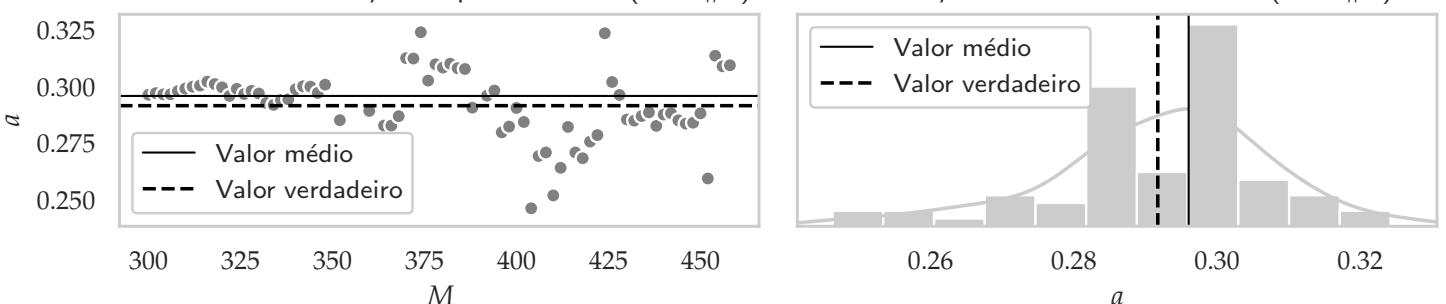

Sensibilidade em função do parâmetro $M$ (Pico \#4).

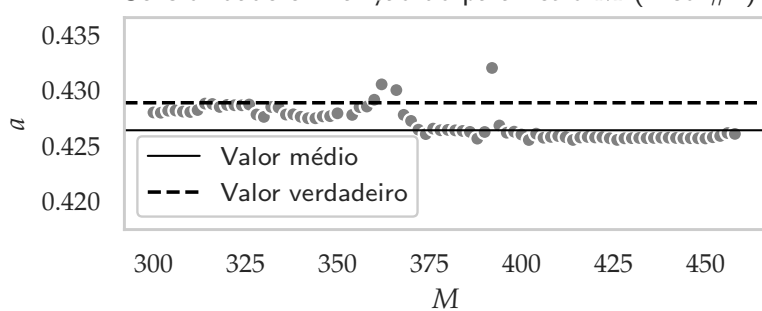

Distribuição. Erro relativo: $0.579 \%$ (Pico \#4).

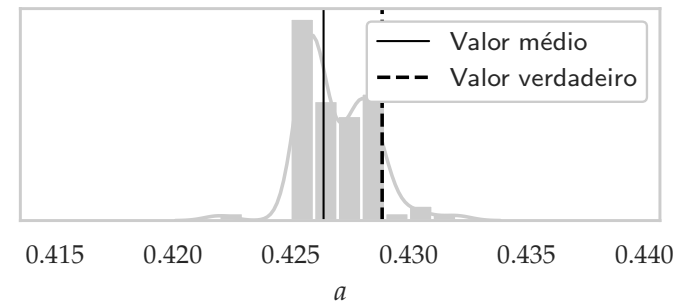

Sensibilidade em função do parâmetro $M$ (Pico \#6).

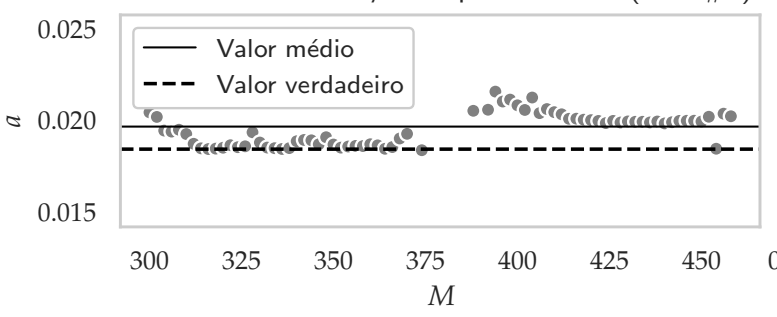

Distribuição. Erro relativo: 6.711\% (Pico \#6).

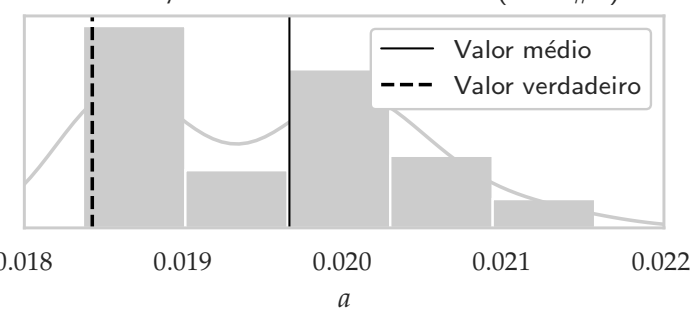

Figura 3.1 - Demonstração do uso de promediação da amplitude $\left(a_{k}\right)$ de $W=80$ linhas estimadas pelo $\operatorname{KBDM}(M=300,302, \ldots, 458)$ em sinais ruidosos $(\sigma=0.004)$ para os picos 1,2 , 3,4 e 6 da Tabela 2.1. As linhas foram agrupadas de maneira supervisionada através dos melhores valores obtidos para o critério de similaridade entre os componentes estimados e verdadeiros.

Fonte: Elaborada pelo autor. 
Sensibilidade em função do parâmetro $M$ (Pico \#7).

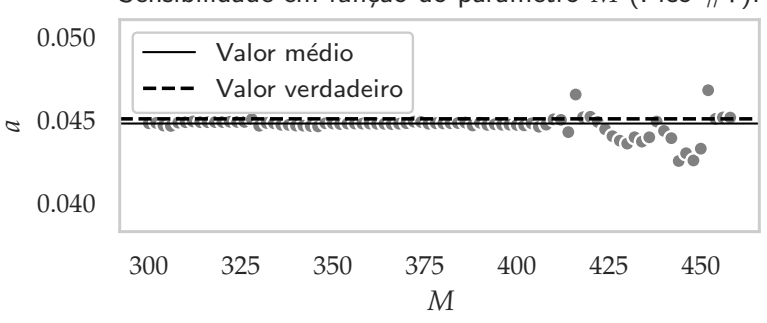

Distribuição. Erro relativo: $0.626 \%$ (Pico \#7)

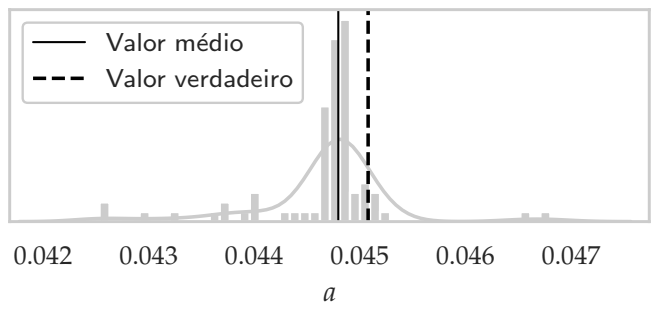

Sensibilidade em função do parâmetro $M$ (Pico \#8).

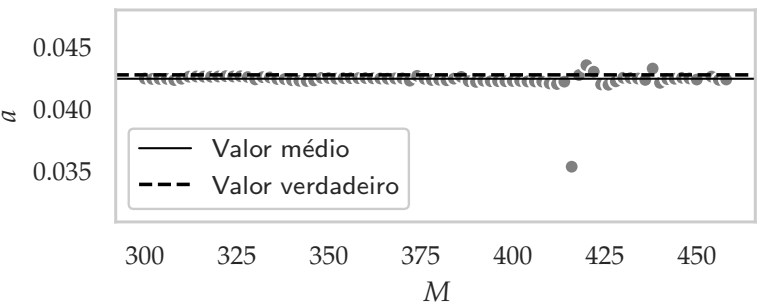

Distribuição. Erro relativo: $0.731 \%$ (Pico \#8).

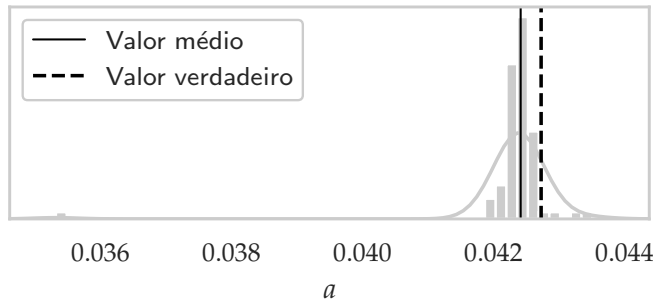

Sensibilidade em função do parâmetro $M$ (Pico \#9).

Distribuição. Erro relativo: $0.027 \%$ (Pico \#9).
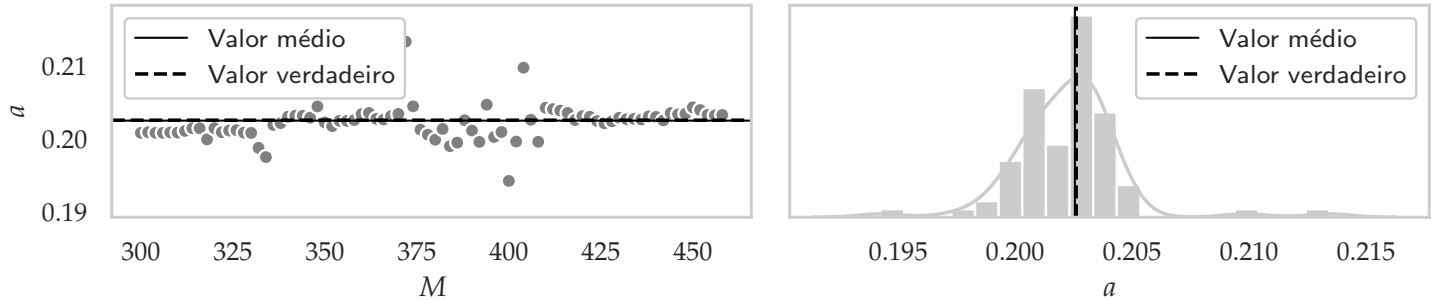

Sensibilidade em função do parâmetro $M$ (Pico \#10)

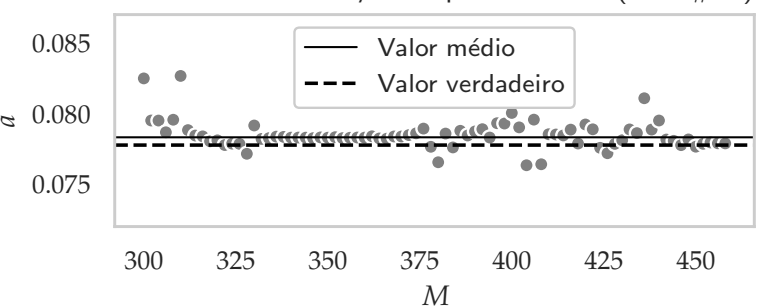

Distribuição. Erro relativo: $0.712 \%$ (Pico \#10).
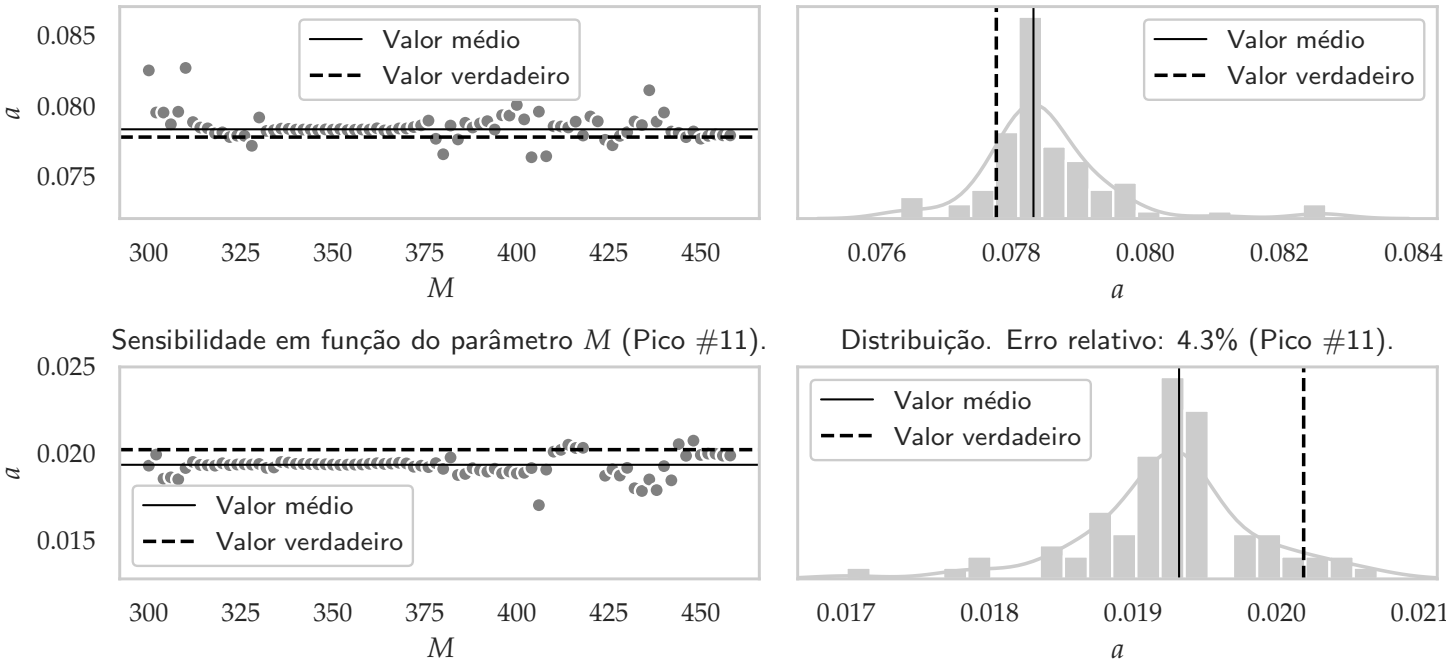

Distribuição. Erro relativo: 4.3\% (Pico \#11).

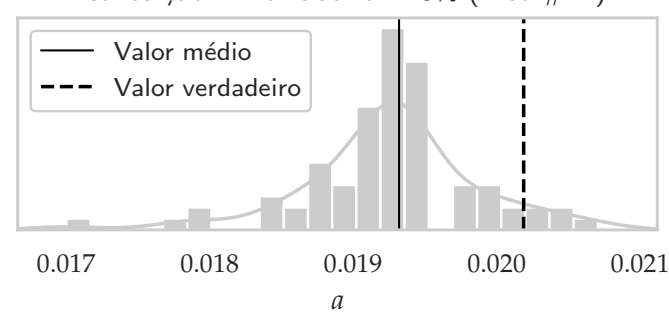

Figura 3.2 - Demonstração do uso de promediação da amplitude $\left(a_{k}\right)$ de $W=80$ linhas estimadas pelo $\operatorname{KBDM}(M=300,302, \ldots, 458)$ em sinais ruidosos $(\sigma=0.004)$ para os picos 7,8 , 9,10 e 11 da Tabela 2.1. As linhas foram agrupadas de maneira supervisionada através dos melhores valores obtidos para o critério de similaridade entre os componentes estimados e verdadeiros.

Fonte: Elaborada pelo autor. 


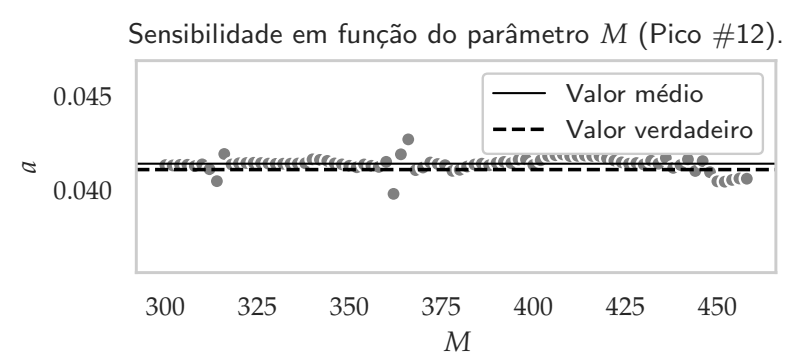

Distribuição. Erro relativo: 0.762\% (Pico \#12).
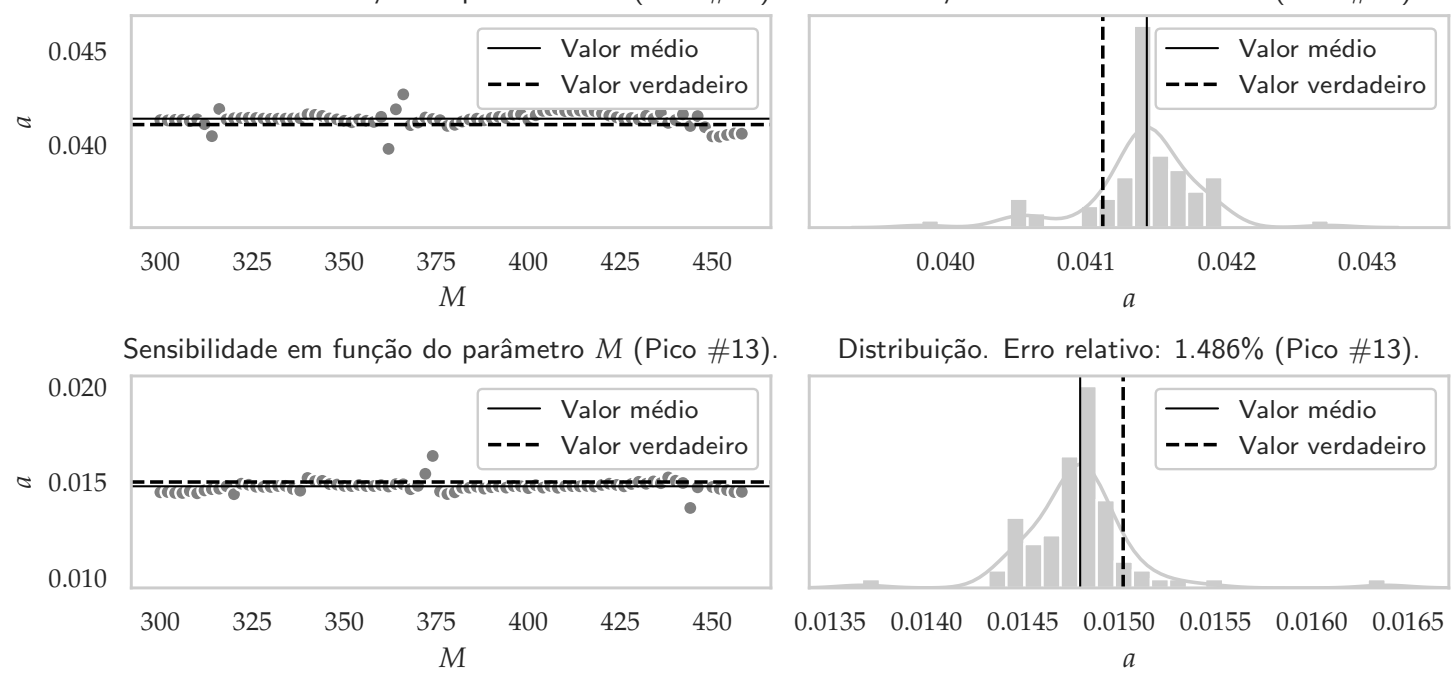

Distribuição. Erro relativo: 1.486\% (Pico \#13).

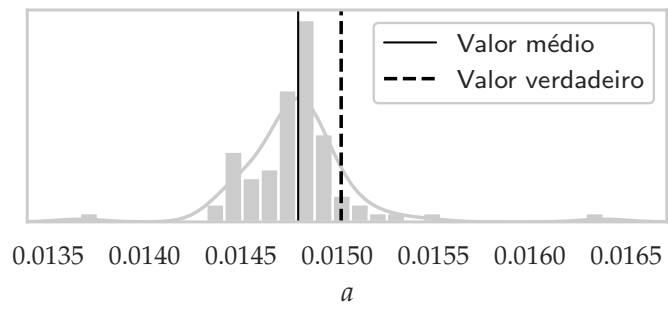

Sensibilidade em função do parâmetro $M$ (Pico \#14).

Distribuição. Erro relativo: 1.078\% (Pico \#14).
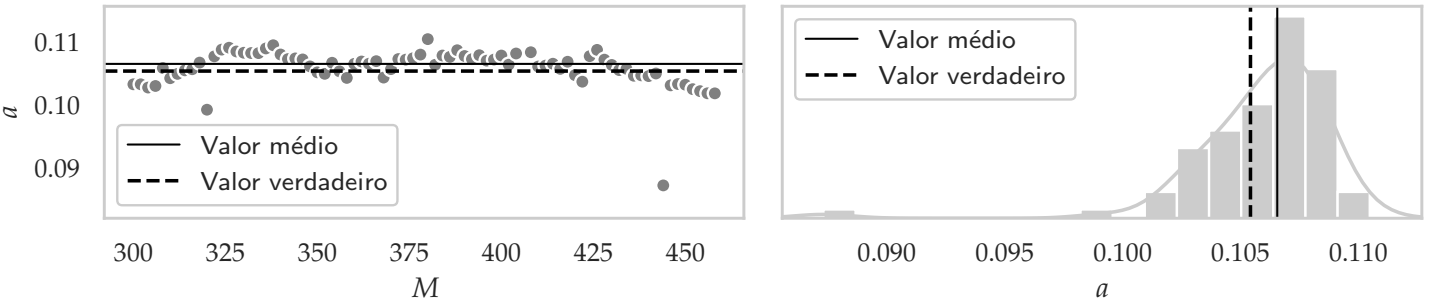

Sensibilidade em função do parâmetro $M$ (Pico \#15).

Distribuição. Erro relativo: 0.006\% (Pico \#15).
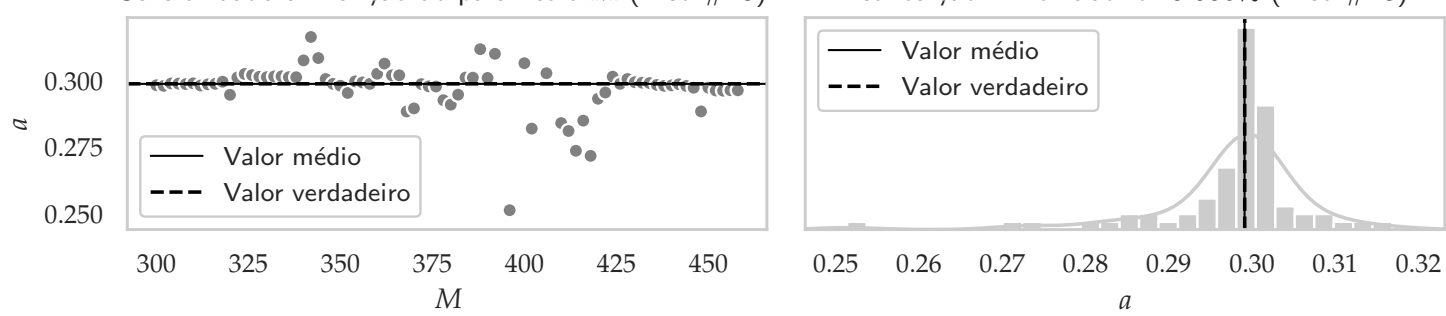

Sensibilidade em função do parâmetro $M$ (Pico \#16)

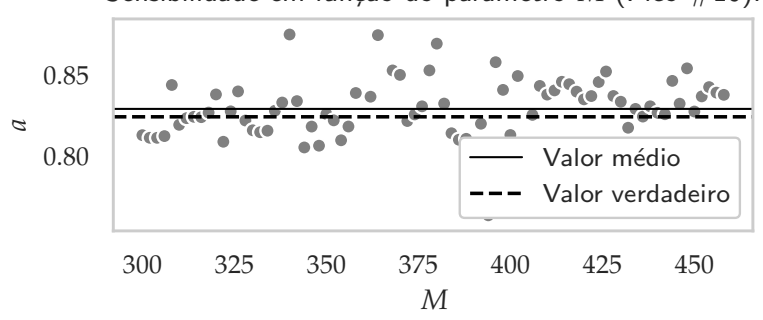

Distribuição. Erro relativo: 0.594\% (Pico \#16).

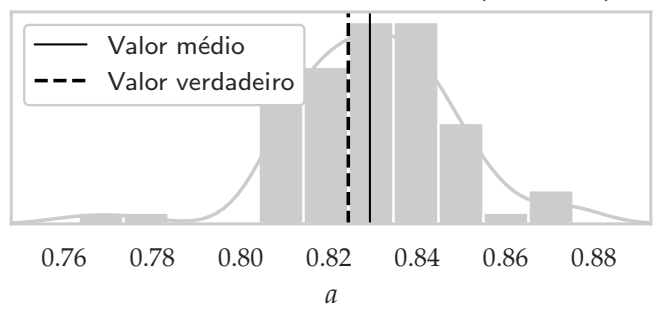

Figura 3.3 - Demonstração do uso de promediação da amplitude $\left(a_{k}\right)$ de $W=80$ linhas estimadas pelo $\mathrm{KBDM}(M=300,302, \ldots, 458)$ em sinais ruidosos $(\sigma=0.004)$ para os picos $12,13,14,15$ e 16 da Tabela 2.1. As linhas foram agrupadas de maneira supervisionada através dos melhores valores obtidos para o critério de similaridade entre os componentes estimados e verdadeiros.

Fonte: Elaborada pelo autor. 

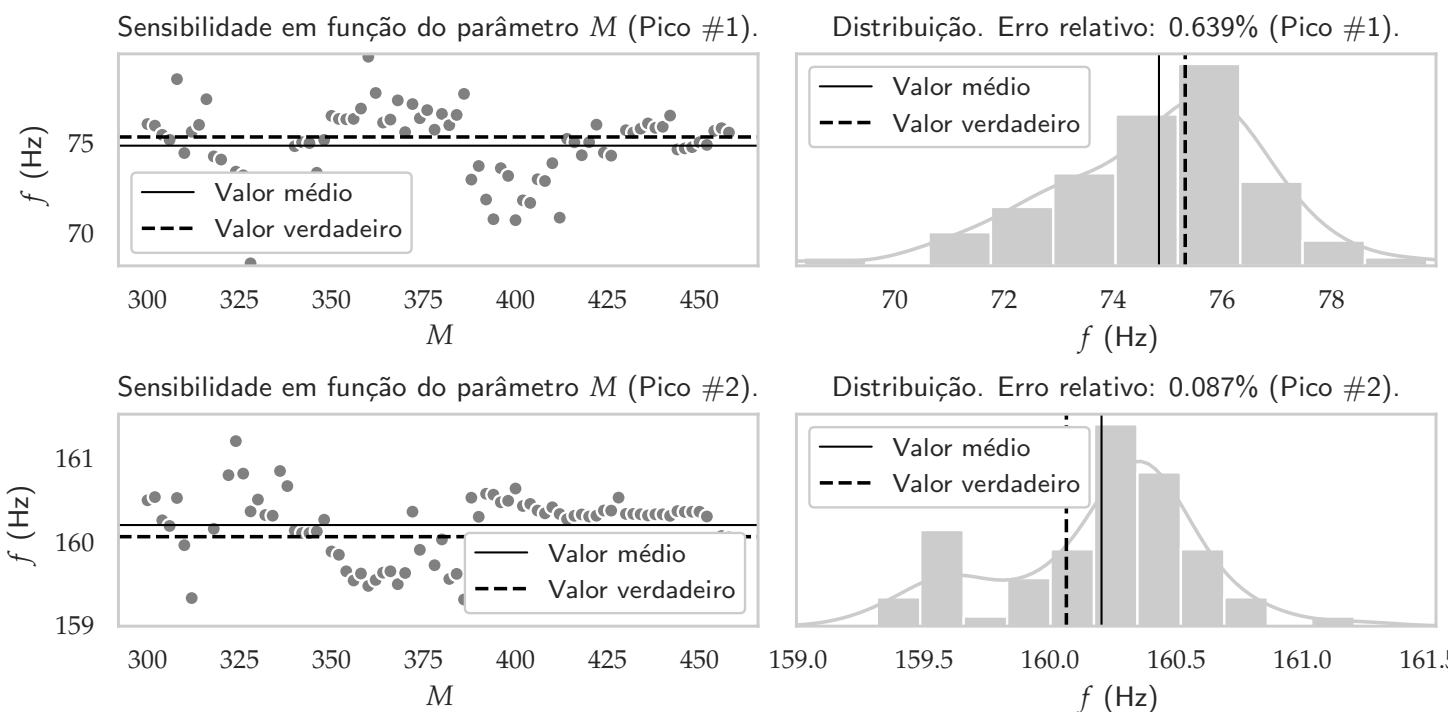

Distribuição. Erro relativo: 0.087\% (Pico \#2).
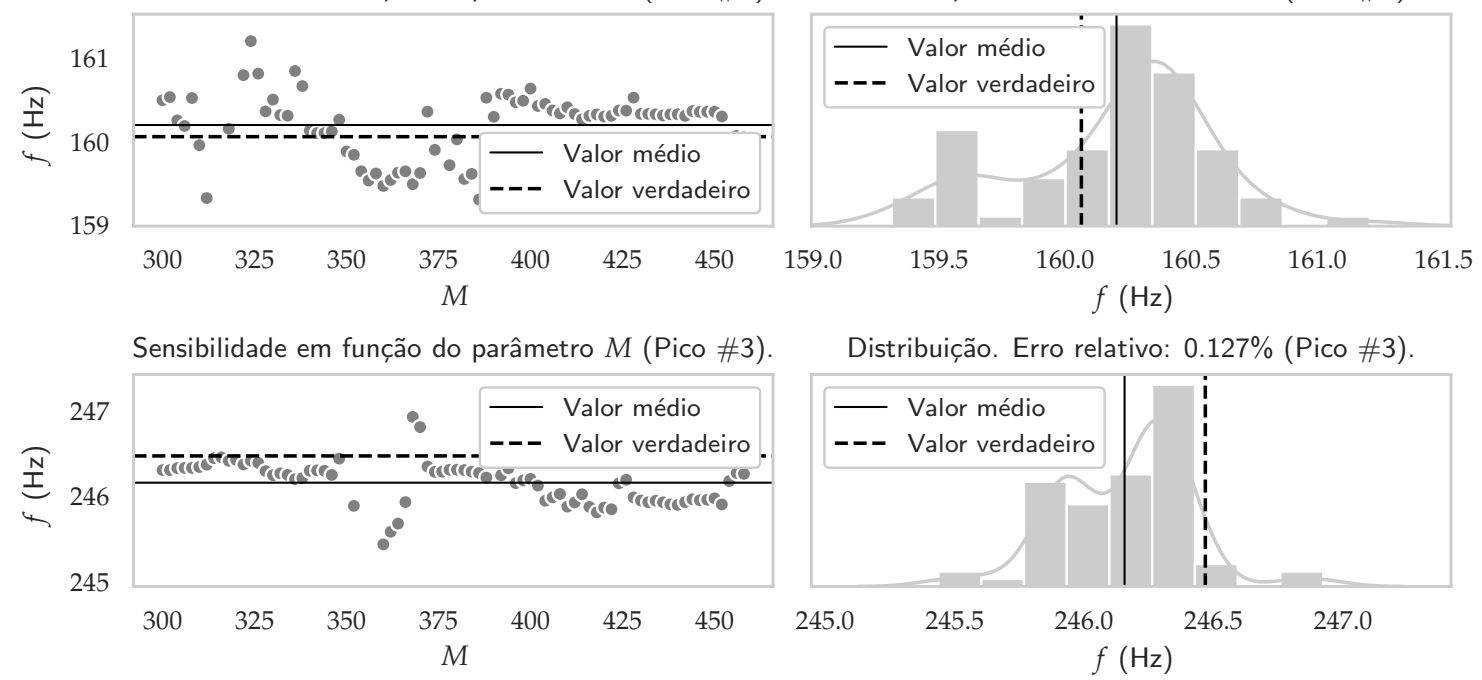

Distribuição. Erro relativo: $0.127 \%$ (Pico \#3).
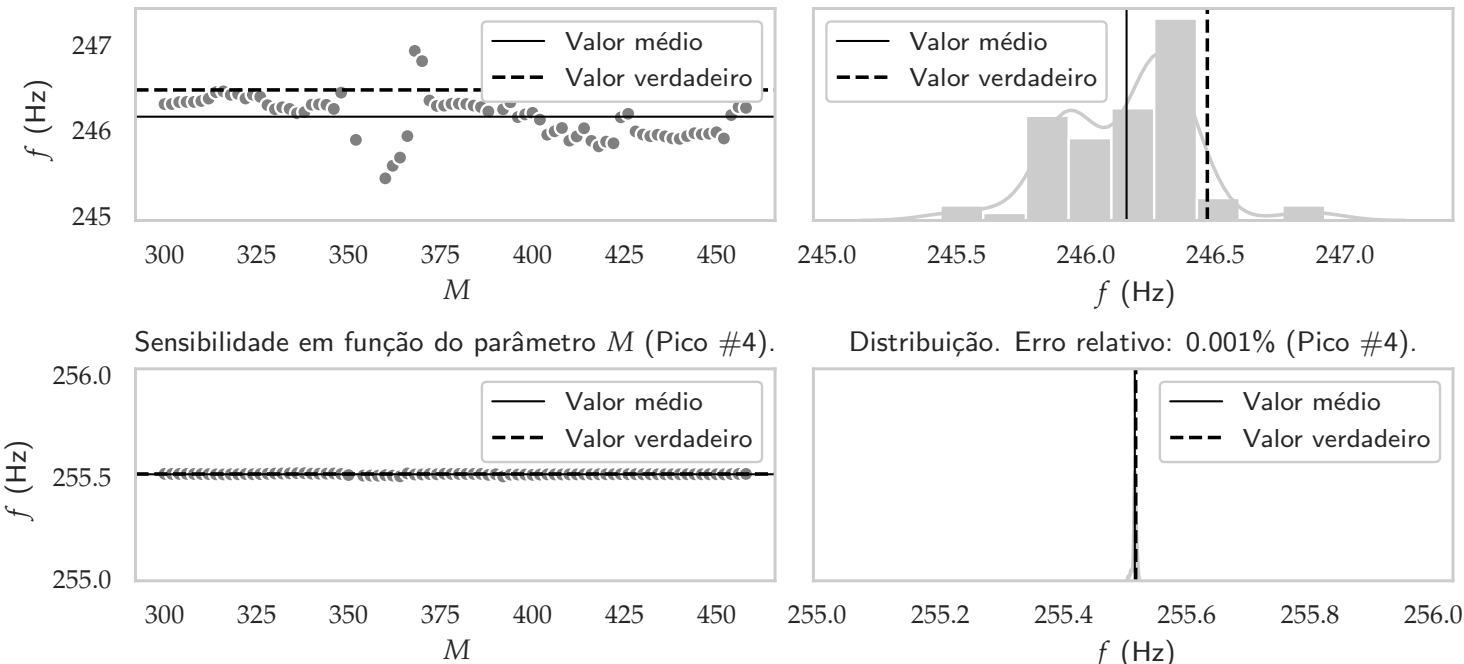

Distribuição. Erro relativo: 0.001\% (Pico \#4).

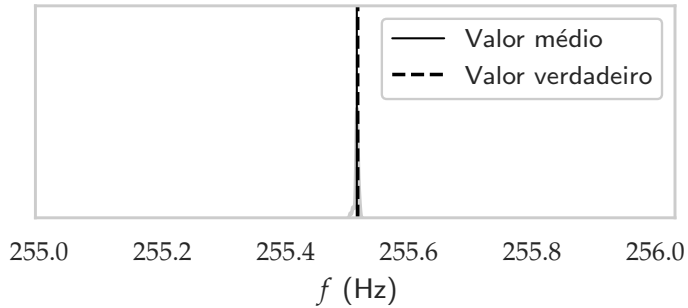

Sensibilidade em função do parâmetro $M$ (Pico \#6).

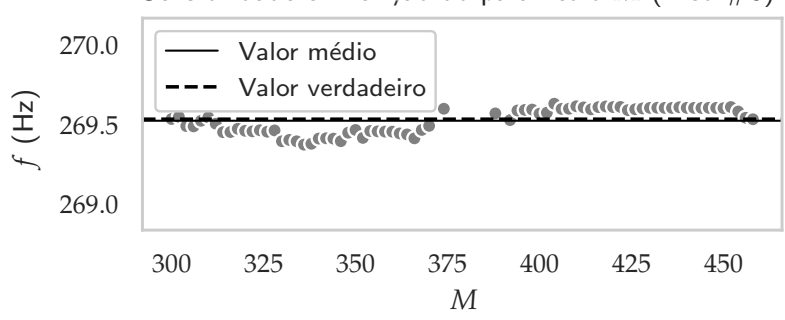

Distribuição. Erro relativo: 0.004\% (Pico \#6).

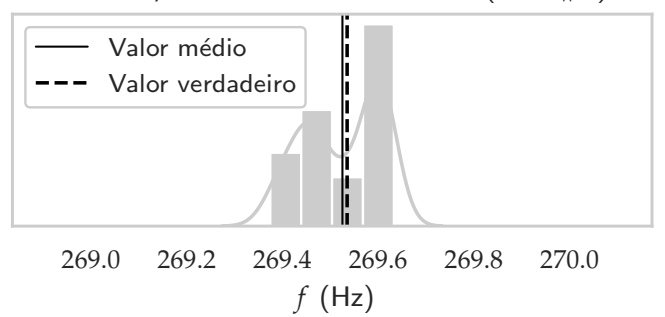

Figura 3.4 - Demonstração do uso de promediação da frequência $\left(f_{k}\right)$ de $W=80$ linhas estimadas pelo $\operatorname{KBDM}(M=300,302, \ldots, 458)$ em sinais ruidosos $(\sigma=0.004)$ para os picos 1,2 , 3, 4 e 6 da Tabela 2.1. As linhas foram agrupadas de maneira supervisionada através dos melhores valores obtidos para o critério de similaridade entre os componentes estimados e verdadeiros.

Fonte: Elaborada pelo autor. 

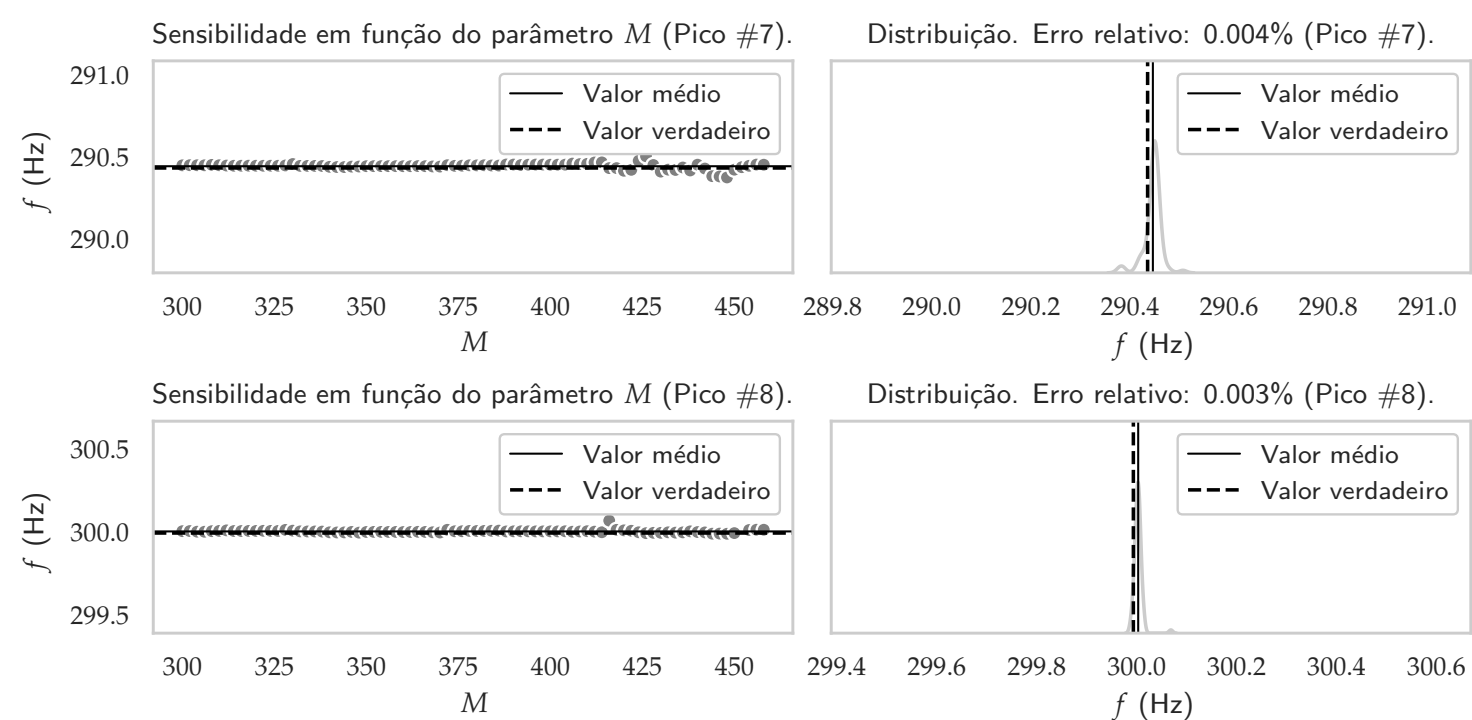

Distribuição. Erro relativo: $0.003 \%$ (Pico \#8).
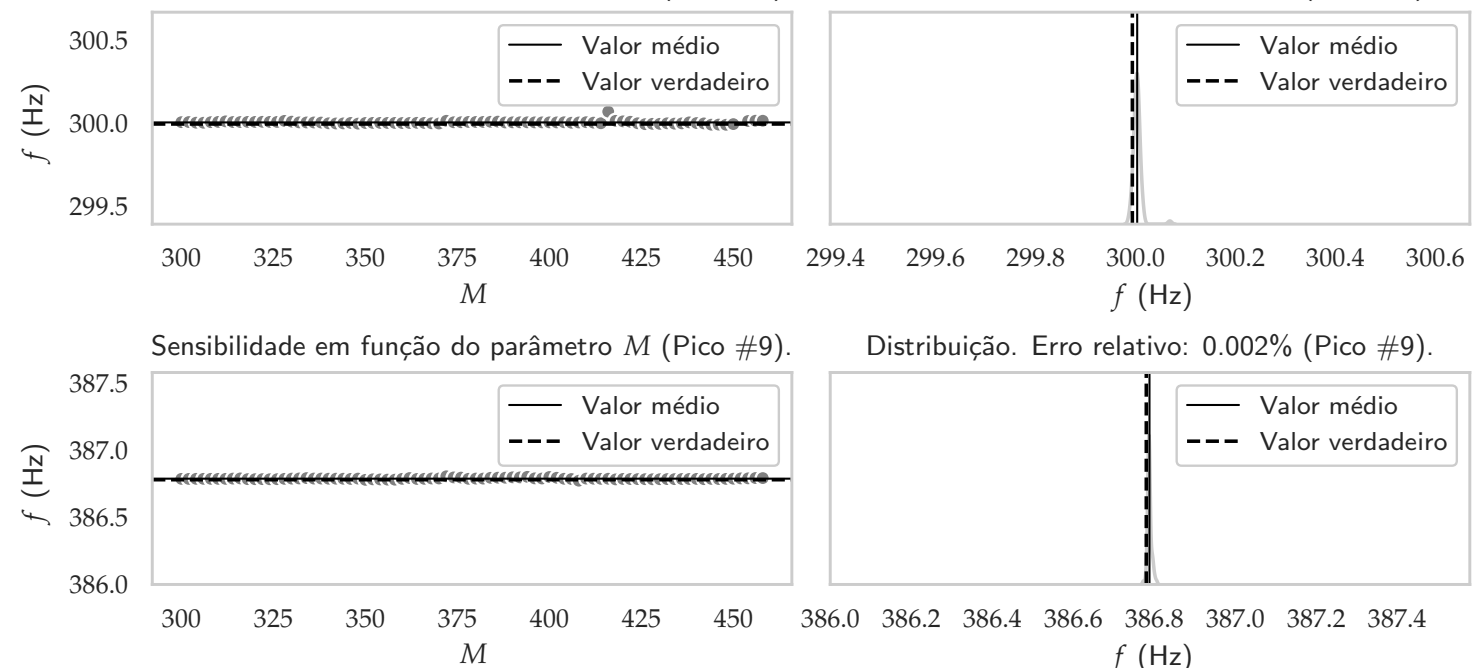

Distribuição. Erro relativo: $0.002 \%$ (Pico \#9).

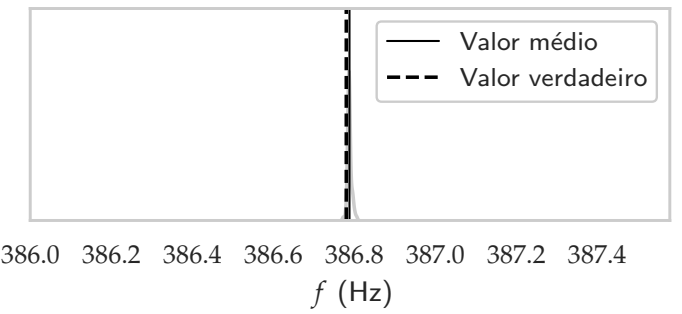

Sensibilidade em função do parâmetro $M$ (Pico \#10).

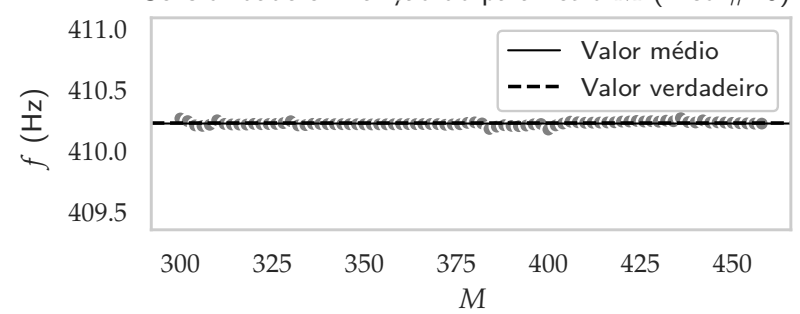

Distribuição. Erro relativo: 0.001\% (Pico \#10).

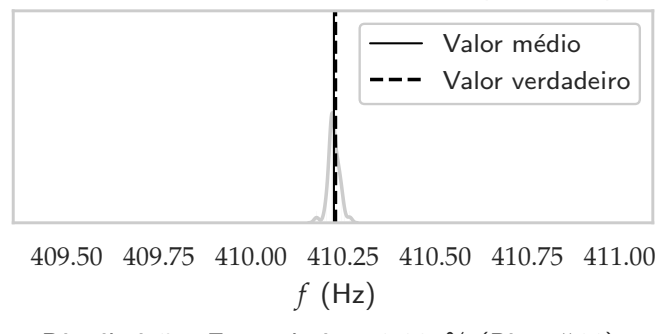

Sensibilidade em função do parâmetro $M$ (Pico \#11).

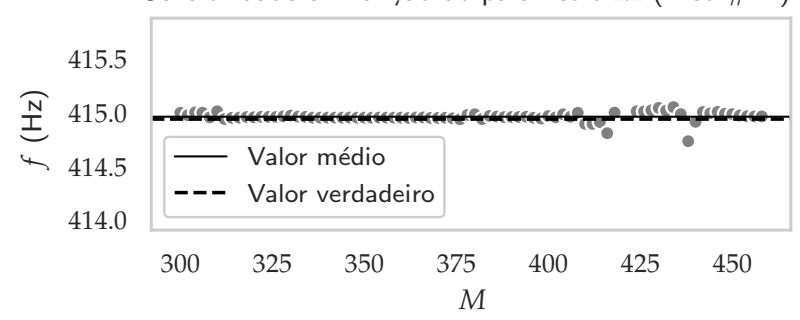

Distribuição. Erro relativo: 0.005\% (Pico \#11).



Figura 3.5 - Demonstração do uso de promediação da frequência $\left(f_{k}\right)$ de $W=80$ linhas estimadas pelo $\operatorname{KBDM}(M=300,302, \ldots, 458)$ em sinais ruidosos $(\sigma=0.004)$ para os picos 7,8 , 9,10 e 11 da Tabela 2.1. As linhas foram agrupadas de maneira supervisionada através dos melhores valores obtidos para o critério de similaridade entre os componentes estimados e verdadeiros.

Fonte: Elaborada pelo autor. 


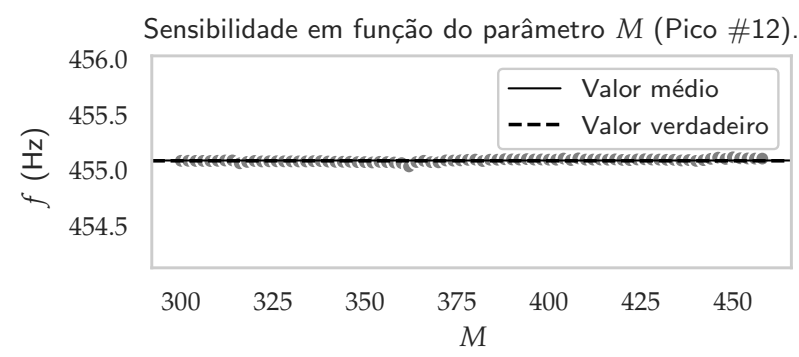

Distribuição. Erro relativo: $0.001 \%$ (Pico \#12).
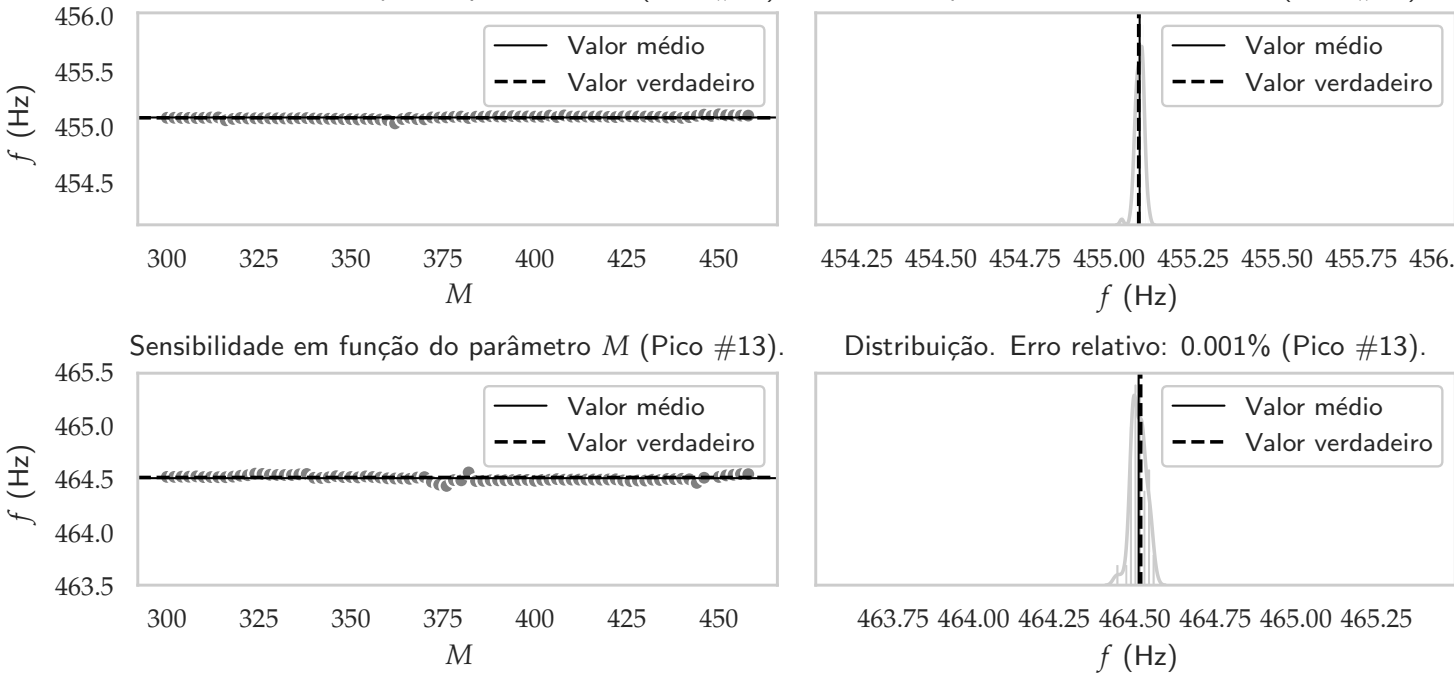

454.25454 .50454 .75455 .00455 .25455 .50455 .75456 .00 $f(\mathrm{~Hz})$

Distribuição. Erro relativo: 0.001\% (Pico \#13).

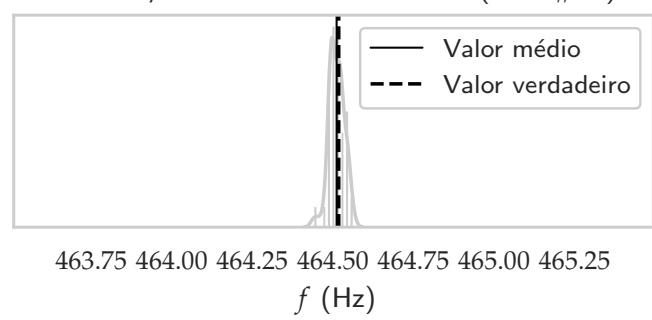

Sensibilidade em função do parâmetro $M$ (Pico \#14).

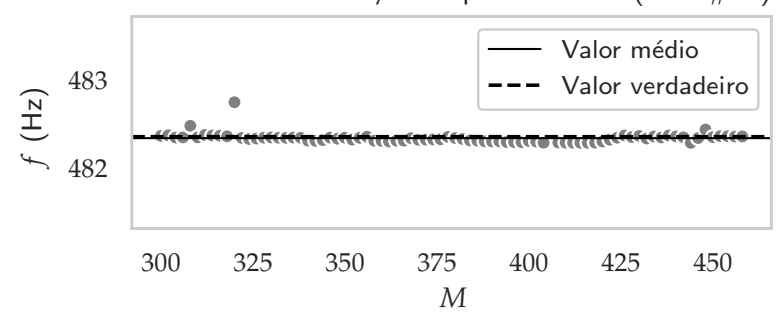

Distribuição. Erro relativo: $0.003 \%$ (Pico \#14).

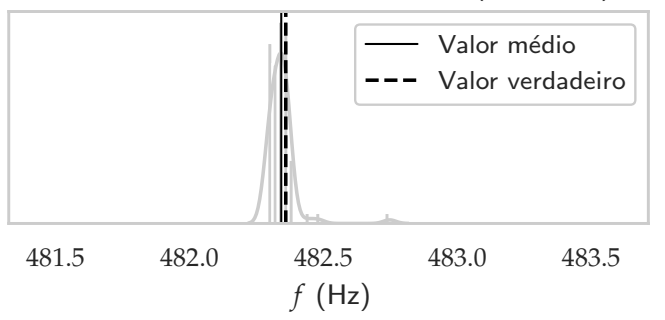

Sensibilidade em função do parâmetro $M$ (Pico \#15)

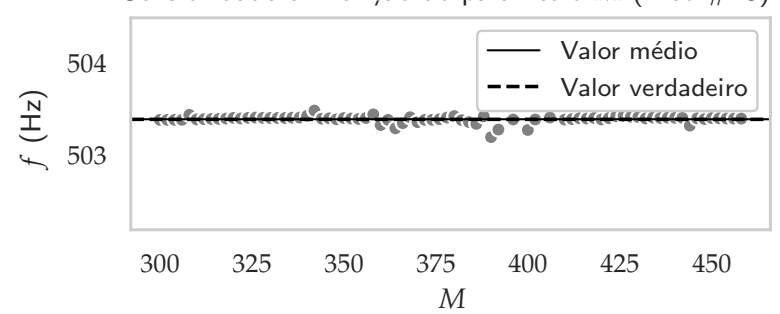

Distribuição. Erro relativo: 0.0\% (Pico \#15)

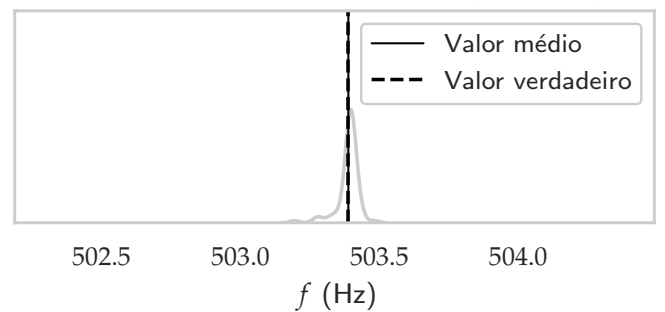

Sensibilidade em função do parâmetro $M$ (Pico \#16).

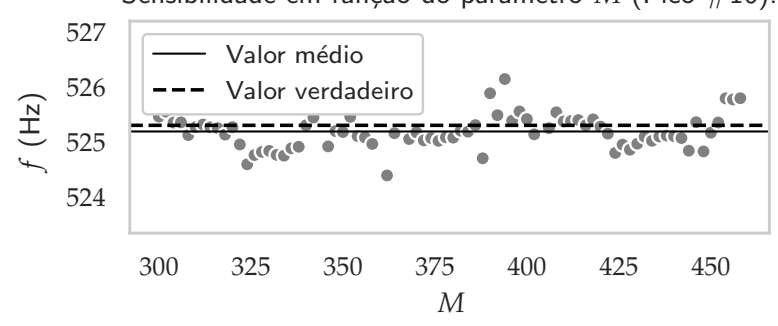

Distribuição. Erro relativo: 0.021\% (Pico \#16).

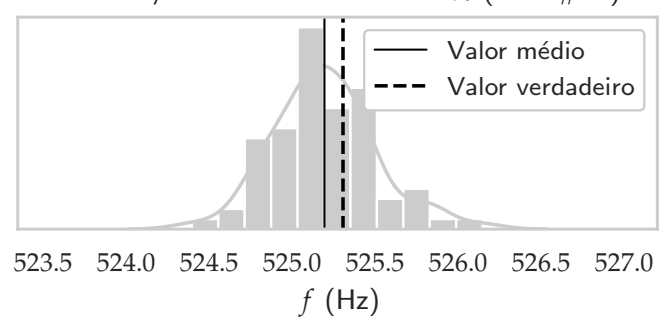

Figura 3.6 - Demonstração do uso de promediação da frequência $\left(f_{k}\right)$ de $W=80$ linhas estimadas pelo $\operatorname{KBDM}(M=300,302, \ldots, 458)$ em sinais ruidosos $(\sigma=0.004)$ para os picos $12,13,14,15$ e 16 da Tabela 2.1. As linhas foram agrupadas de maneira supervisionada através dos melhores valores obtidos para o critério de similaridade entre os componentes estimados e verdadeiros.

Fonte: Elaborada pelo autor. 


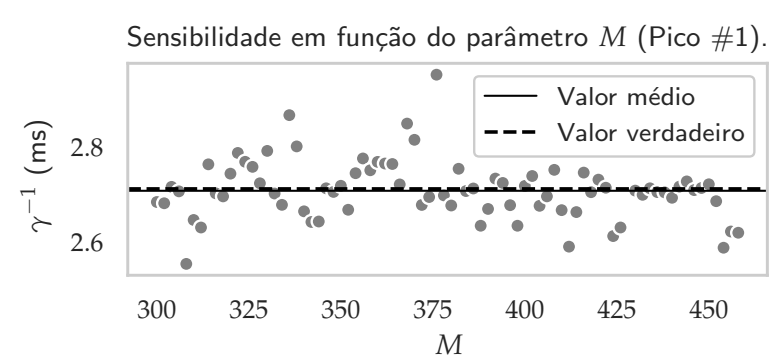

Distribuição. Erro relativo: $0.147 \%$ (Pico \#1).

Sensibilidade em função do parâmetro $M$ (Pico \#2).
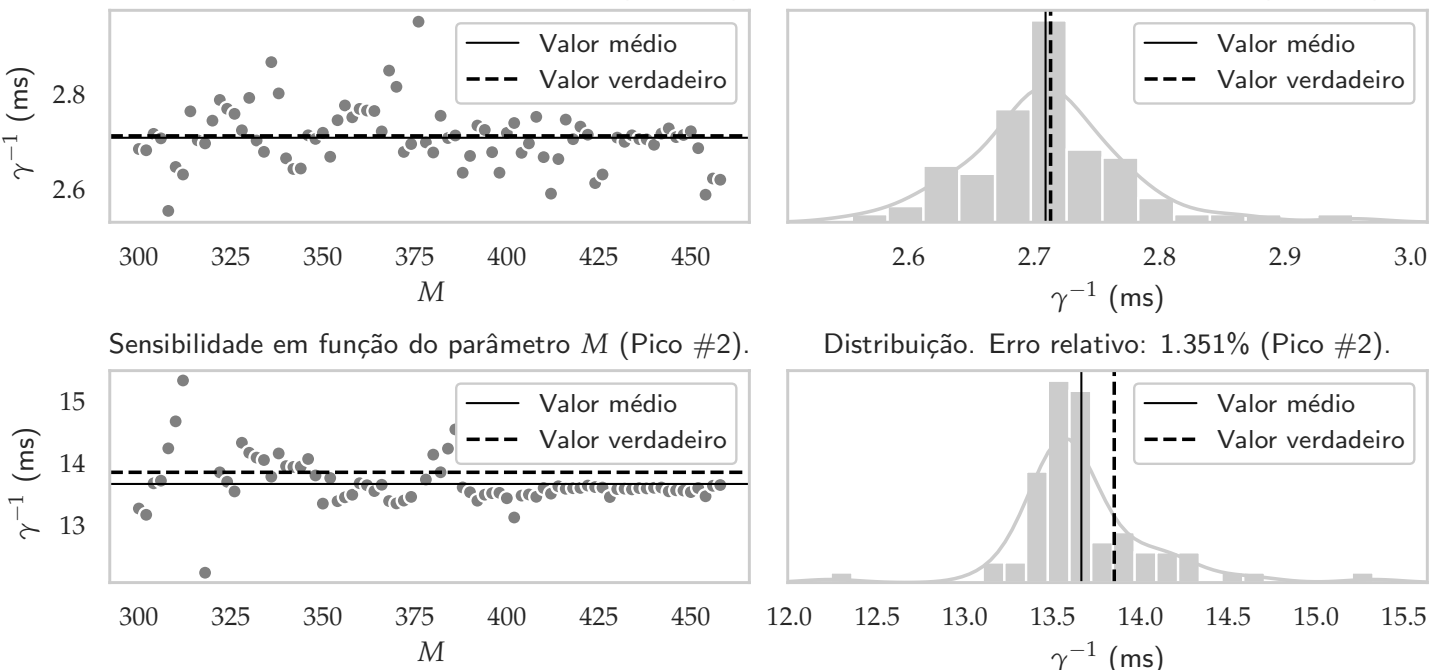

Distribuição. Erro relativo: $1.351 \%$ (Pico \#2).

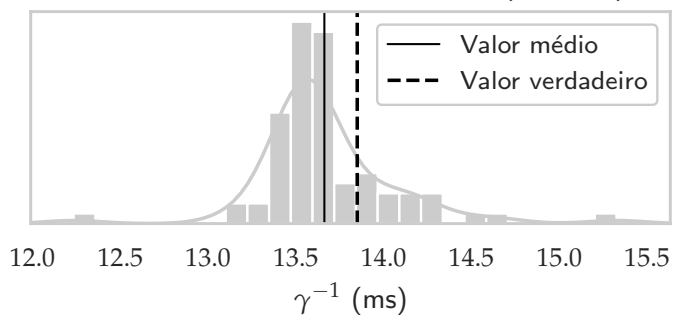

Sensibilidade em função do parâmetro $M$ (Pico \#3).

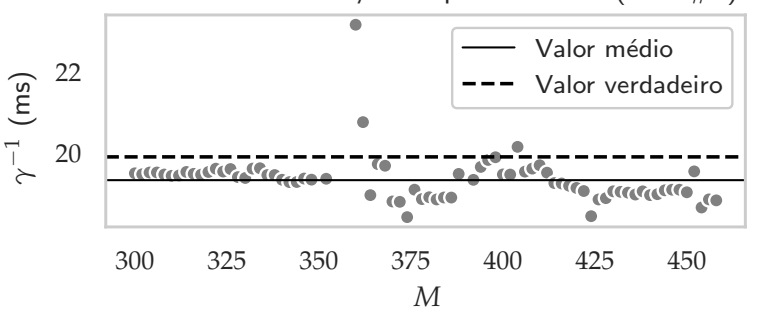

Distribuição. Erro relativo: $2.869 \%$ (Pico \#3).

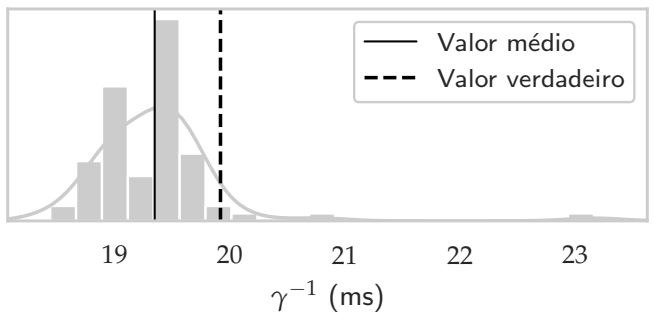

Sensibilidade em função do parâmetro $M$ (Pico \#4)

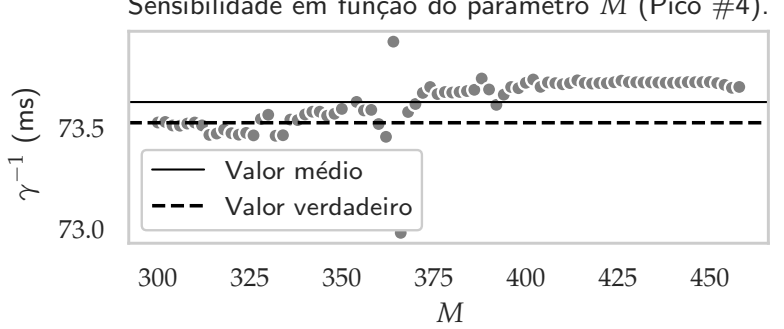

Distribuição. Erro relativo: $0.14 \%$ (Pico \#4).

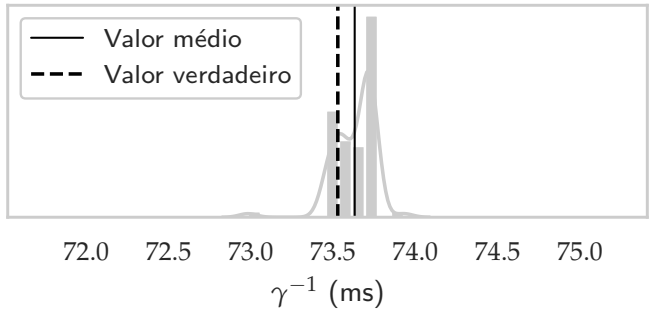

Sensibilidade em função do parâmetro $M$ (Pico \#6).

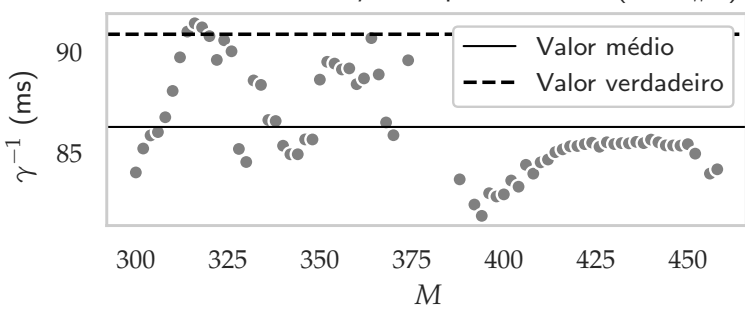

Distribuição. Erro relativo: 5.082\% (Pico \#6)

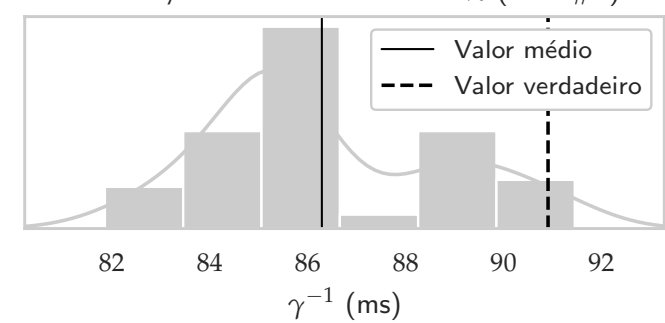

Figura 3.7 - Demonstração do uso de promediação da taxa de relaxação $\left(\gamma_{k}\right)$ de $W=80$ linhas estimadas pelo $\mathrm{KBDM}(M=300,302, \ldots, 458)$ em sinais ruidosos $(\sigma=0.004)$ para os picos 1, 2, 3, 4 e 6 da Tabela 2.1. As linhas foram agrupadas de maneira supervisionada através dos melhores valores obtidos para o critério de similaridade entre os componentes estimados e verdadeiros.

Fonte: Elaborada pelo autor. 

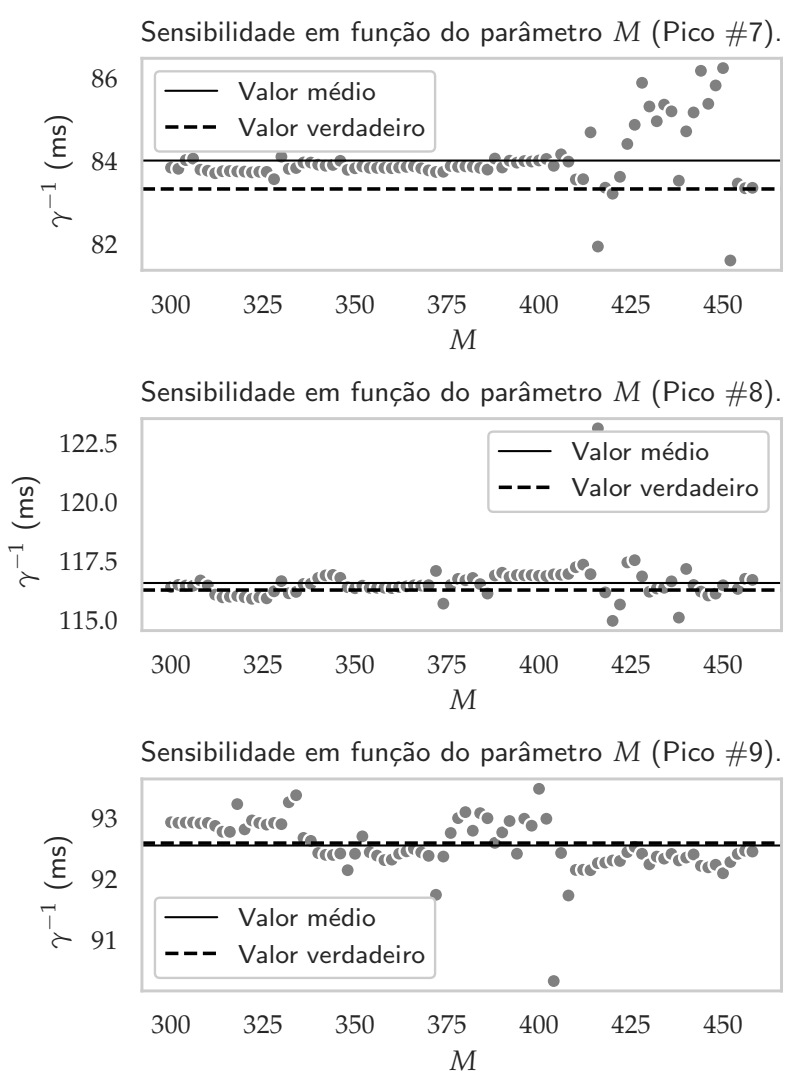

Sensibilidade em função do parâmetro $M$ (Pico \#10)
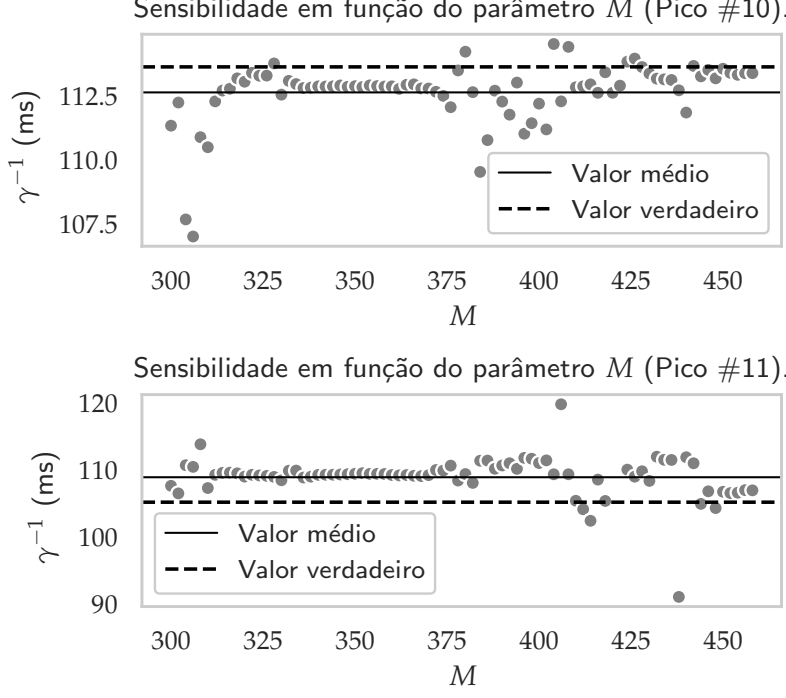

Distribuição. Erro relativo: $0.823 \%$ (Pico \#7).

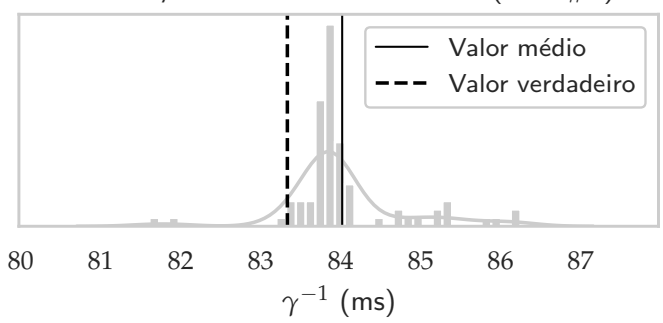

Distribuição. Erro relativo: $0.257 \%$ (Pico \#8).

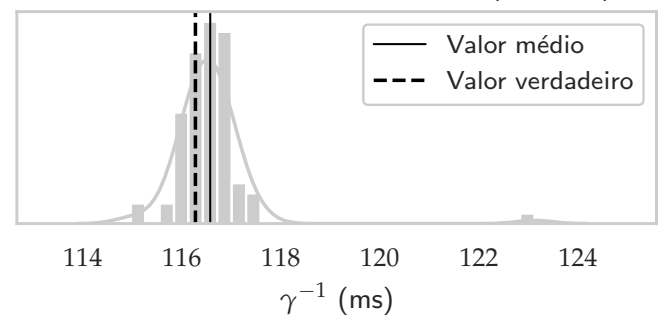

Distribuição. Erro relativo: $0.043 \%$ (Pico \#9).

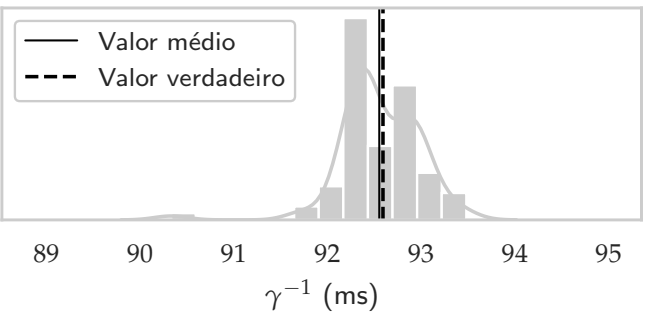

Distribuição. Erro relativo: $0.877 \%$ (Pico \#10).

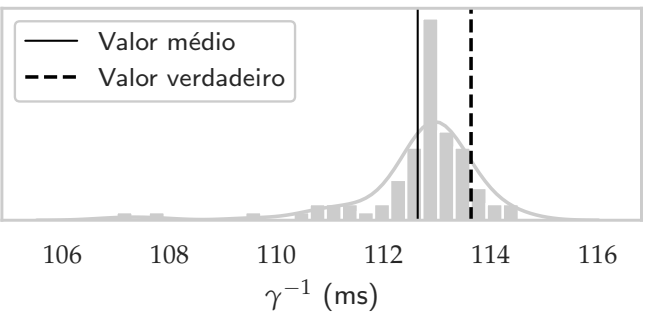

Distribuição. Erro relativo: 3.54\% (Pico \#11).

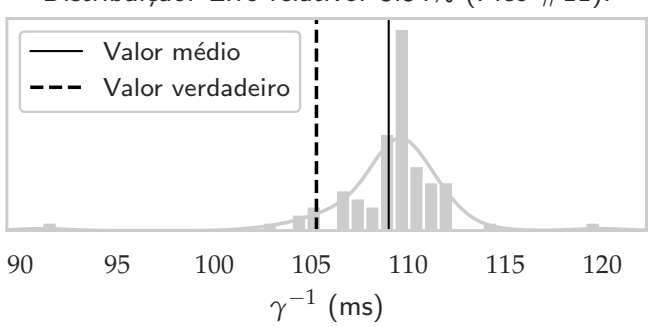

Figura 3.8 - Demonstração do uso de promediação da taxa de relaxação $\left(\gamma_{k}\right)$ de $W=80$ linhas estimadas pelo $\operatorname{KBDM}(M=300,302, \ldots, 458)$ em sinais ruidosos $(\sigma=0.004)$ para os picos 7, 8, 9, 10 e 11 da Tabela 2.1. As linhas foram agrupadas de maneira supervisionada através dos melhores valores obtidos para o critério de similaridade entre os componentes estimados e verdadeiros.

Fonte: Elaborada pelo autor. 


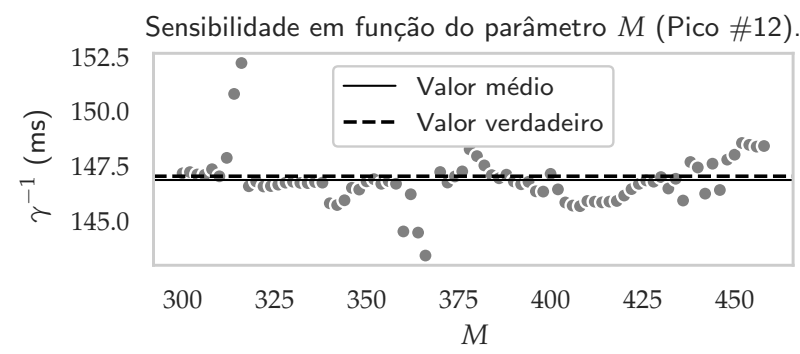

Distribuição. Erro relativo: $0.117 \%$ (Pico \#12).
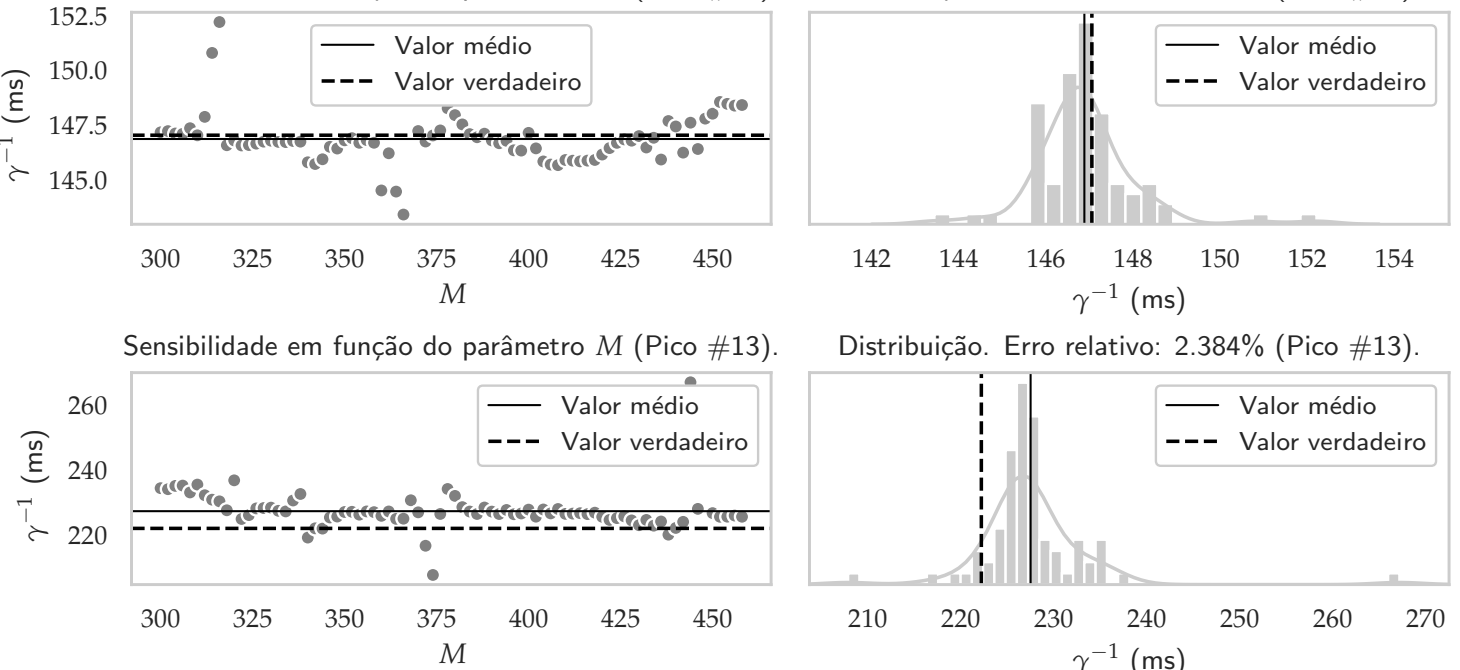

Distribuição. Erro relativo: $2.384 \%$ (Pico \#13).

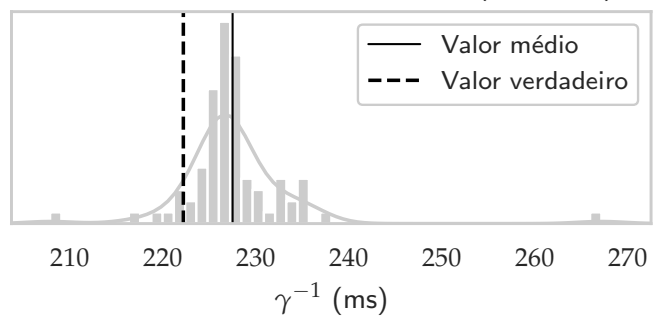

Sensibilidade em função do parâmetro M (Pico \#14).

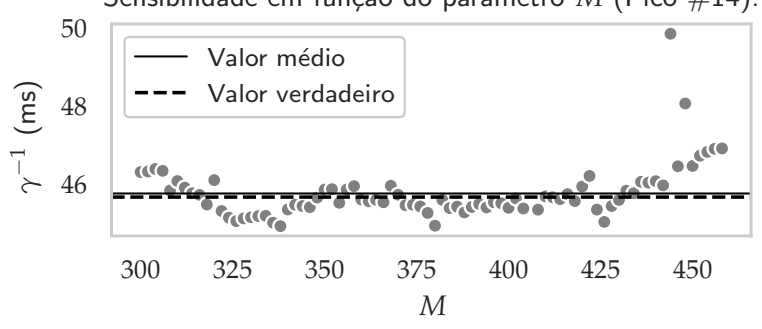

Distribuição. Erro relativo: $0.201 \%$ (Pico \#14).

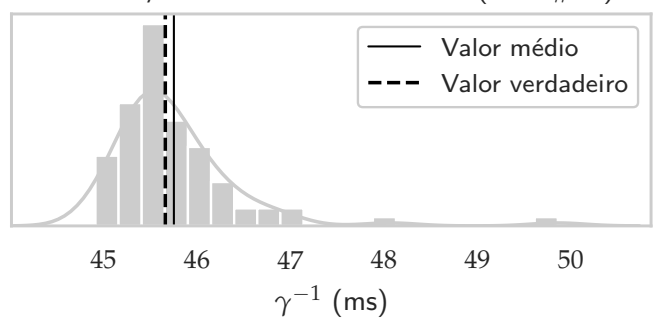

Sensibilidade em função do parâmetro M (Pico \#15)

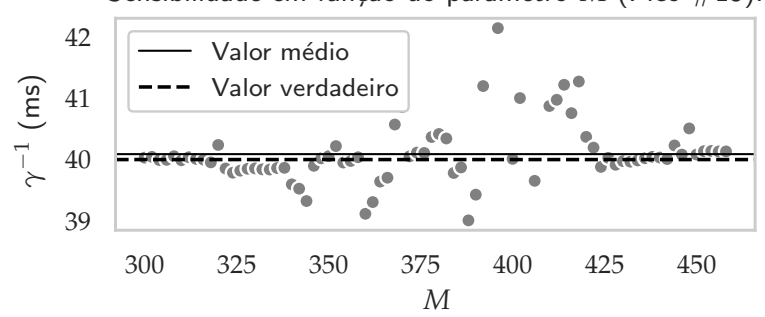

Distribuição. Erro relativo: $0.221 \%$ (Pico \#15).

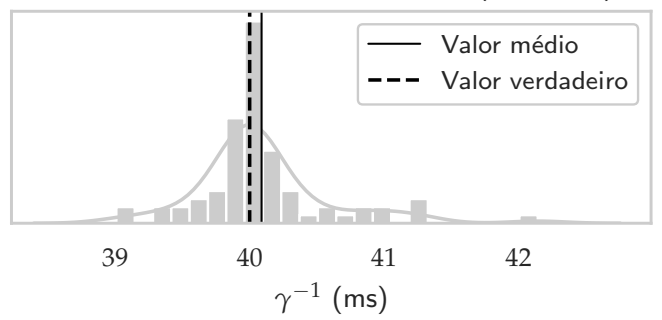

Sensibilidade em função do parâmetro $M$ (Pico \#16).

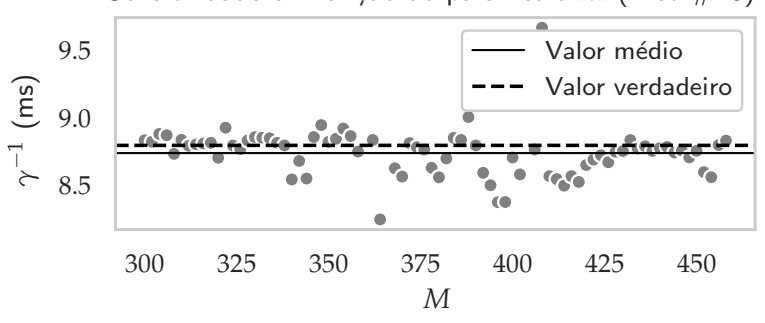

Distribuição. Erro relativo: $0.651 \%$ (Pico \#16).

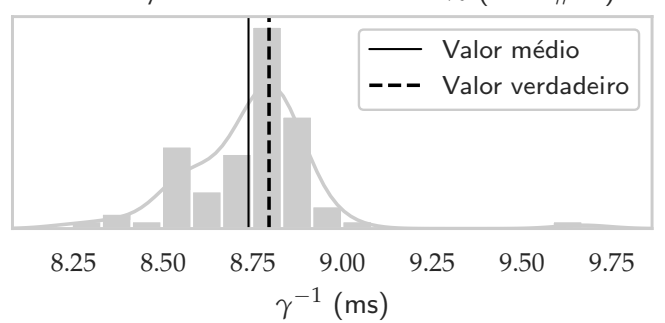

Figura 3.9 - Demonstração do uso de promediação da taxa de relaxação $\left(\gamma_{k}\right)$ de $W=80$ linhas estimadas pelo $\mathrm{KBDM}(M=300,302, \ldots, 458)$ em sinais ruidosos $(\sigma=0.004)$ para os picos 12,13,14, 15 e 16 da Tabela 2.1. As linhas foram agrupadas de maneira supervisionada através dos melhores valores obtidos para o critério de similaridade entre os componentes estimados e verdadeiros.

Fonte: Elaborada pelo autor. 

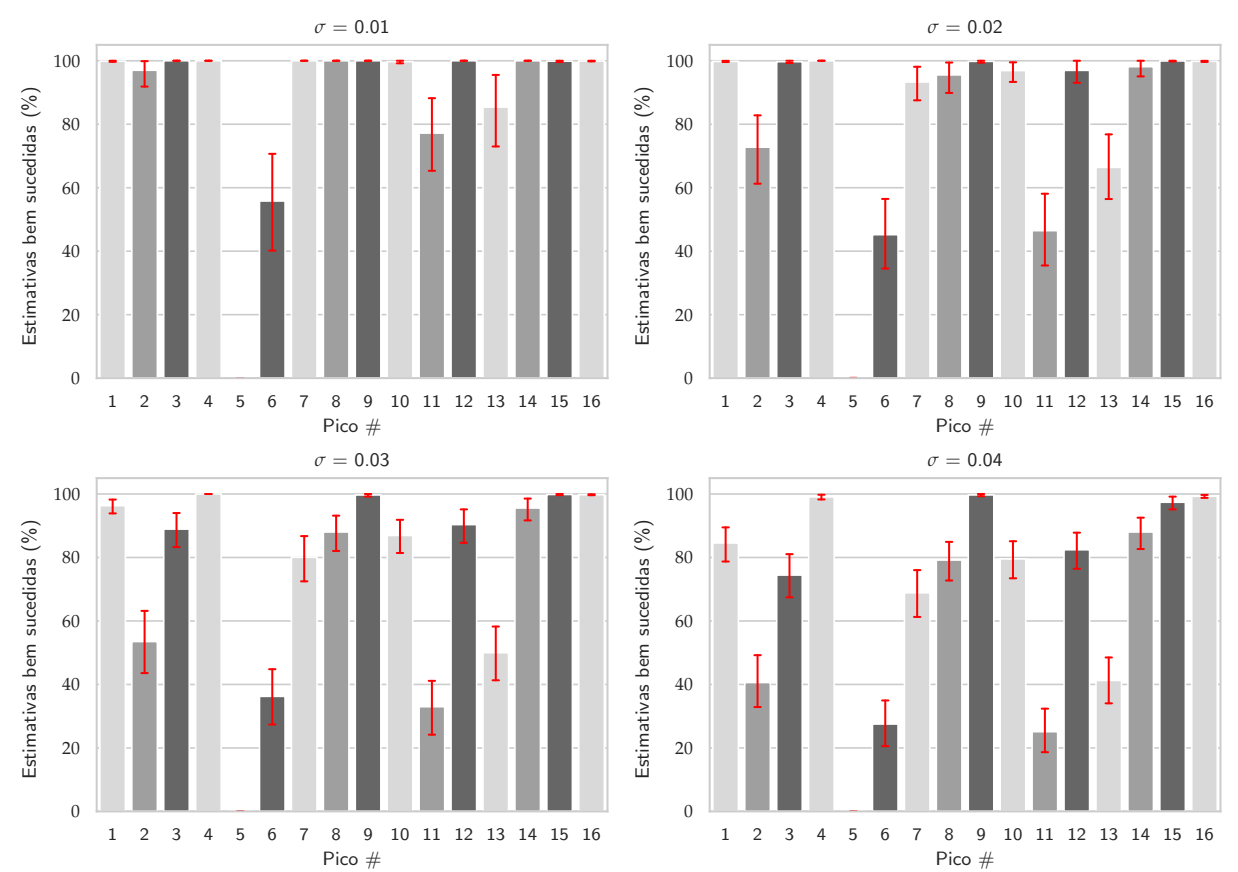

Figura 3.10 - Exemplo do perfil das taxas de reconstruções bem sucedidas dos picos da Tabela 2.1 para o sinal da Figura 2.1 em função do nível de ruído. As barras cinzas verticais representam valores médios das taxas de estimativas bem sucedidas (Equação 3.5) para 30 realizações contendo cada nível de ruído $(\sigma=0.01,0.02,0.04,0.08)$. As barras vermelhas representam o desvio padrão. As amostras foram obtidas através de múltiplos truncamentos do sinal $(M=300,302, \ldots, 458$ e $W=80)$.

Fonte: Elaborada pelo autor.

\subsection{Agrupamento}

\subsubsection{Algoritmos de agrupamento de dados}

Os benefícios da reconstrução das linhas a partir da promediação de várias estimativas obtidas pelo KBDM utilizando múltiplos truncamentos apontados na seção anterior são, na verdade, idealizações do que se pode obter na prática. Embora a análise realizada seja um estudo preliminar importante no entendimento do potencial da técnica da promediação paramétrica, todas as linhas foram agrupadas já se conhecendo o valor correto para cada componente do sinal.

Para viabilizar a técnica da promediação no espaço de parâmetros, o agrupamento das componentes estimadas deve ser realizado de maneira automática levando-se em consideração apenas os critérios de similaridade internos. Ou seja, sem o uso de nenhuma informação 
a priori além do sinal de entrada. Outros métodos amplamente adotados que não utilizam agrupamento de soluções, mas que são baseados no uso de informação a priori podem ser consultados na literatura. $(15,54) \mathrm{O}$ intuito deste trabalho coincide com a abordagem original do KBDM de permitir a estimativa das linhas sem o uso deste tipo de informação.

Há décadas, a análise de agrupamento é um assunto bem abordado na literatura no contexto de reconhecimento de padrões e inteligência artificial. (55-60) Esta ferramenta, também denominada por aprendizado de máquina não supervisionado, permite a detecção automática de estruturas ou padrões em um determinado conjunto de dados. (56) Em particular, a extração de informação a partir de grandes volumes de dados tem sido ponto focal das aplicações desta técnica na área biomédica. (61)

De maneira geral, a análise de agrupamento consiste na organização de coleções de padrões em aglomerados ou clusters com base na similaridade entre os objetos que os constituem. $(56,62)$ A similaridade, portanto, deve ser aferida a partir de características dos objetos que são relevantes ao problema em questão e, para os mesmos objetos, diversos agrupamentos distintos podem ser obtidos a partir da escolha dessas características ou algoritmos selecionados. (60)

A grande diversidade de objetivos no uso de algoritmos de agrupamento de dados resultou em várias definições distintas e, em alguns casos, divergentes para o que se define como sendo um aglomerado ou cluster. $(57,60)$ Algumas definições baseadas em critérios de homogeneidade interna e separação externa reportadas por Xu et al. são: (61)

1. Um cluster é formado por um conjunto de objetos similares entre si; enquanto objetos pertencentes a clusters distintos não são similares.

2. Um cluster é um conjunto de objetos onde a distância entre qualquer objeto ao centroide do respectivo cluster é menor do que a distância até o centroide de qualquer outro cluster.

3. Um cluster é um conjunto de objetos cuja distância entre qualquer par de elementos contidos nele deve ser menor do que a distância entre qualquer objeto deste mesmo cluster até outro não pertencente a ele.

4. Um cluster é uma região contínua de alta densidade de objetos separada por regiões de baixa densidade de objetos.

A obtenção de uma coleção de clusters ou particionamento dos dados se dá através da otimização de um critério de agrupamento. Cada critério de agrupamento está diretamente ligado a uma definição de cluster e mais de um critério de agrupamento pode ser utilizado pelo mesmo algoritmo para satisfazer uma definição específica para cada aglomerado. Os critérios 
de agrupamento envolvem a utilização de medidas de distância, similaridade ou dissimilaridade entre os objetos, além de um método de busca, normalmente definido heuristicamente. Os três principais critérios de agrupamento são a compactação; o encadeamento ou ligação; e a separação espacial.

A compactação é um conceito ligado diretamente à homogeneidade intra-cluster, onde a variação entre os valores dos atributos e as distâncias entre os objetos são menores quanto mais compacto o aglomerado for. Os algoritmos que utilizam este critério de particionamento são eficientes para encontrar agrupamentos hiperesféricos no espaço de dados e possuem dificuldade em detectar estruturas de formato arbitrário.

O critério de encadeamento ou ligação detecta estruturas considerando que dois objetos devem pertencer ao mesmo cluster se eles estiverem próximos ou se existir conectividade entre os mesmos através de outros objetos pertencentes ao mesmo aglomerado. Naturalmente, a ideia deste critério está ligada ao conceito de grafos. Embora o conceito de encadeamento seja eficiente na detecção de formas arbitrárias, ele é sensível à separação espacial entre os aglomerados.

Por fim, o critério da separação espacial leva em consideração apenas a distância entre os clusters. Esta distância pode ser mensurada a partir dos centroides ou as distâncias mínimas ou máximas entre os objetos de cada cluster. Este critério é considerado fraco por produzir soluções triviais se for adotado sozinho e normalmente é utilizado em conjunto a outro critério de agrupamento.

A representação dos objetos através de pontos em um espaço $d$-dimensional, sendo $d$ o número de atributos ou características de cada exemplo, é uma das abordagens mais adotadas. Deste modo, o $i$-ésimo elemento de uma coleção formada por $n$ objetos pode ser descrito através do vetor

$$
\begin{gathered}
\mathbf{x}^{i}=\left(x_{1}, x_{2}, \ldots, x_{d}\right) \\
\mathbf{x}^{i} \in \mathbb{R}^{d}
\end{gathered}
$$

A coleção de objetos pode ser representada através da matriz de dados $\mathcal{X} \in \mathbb{R}^{n \times d}$, onde cada linha desta matriz é constituída pelos vetores $\mathrm{x}^{i}$. Embora a matriz $\mathcal{X}$ seja o requerimento mais comum para os algoritmos de agrupamento de dados, na ausência de atributos para cada exemplo, uma matriz contendo apenas as medidas de similaridades entre cada par de objetos, $\mathcal{S} \in \mathbb{R}^{n \times n}$, pode ser empregada em algumas classes de métodos.

Independente do critério de agrupamento ou definição do cluster, é possível notar a ne- 
cessidade da escolha de uma medida de similaridade ou distância entre dois objetos. De fato, a distância pode ser considerada uma medida de dissimilaridade, que é um conceito inverso ao da similaridade. Embora a distância Euclidiana seja certamente a mais adotada e talvez a mais intuitiva, diversas outras medidas foram propostas ao longo dos anos para cobrir necessidades específicas. (63)

Para além da escolha da melhor representação matemática e das heurísticas de busca de padrões ou estruturas em um determinado conjunto de dados, outros critérios são relevantes na escolha do algoritmo mais apropriado. A eficiência computacional, a complexidade de interpretação e a disponibilidade de implementações confiáveis são fatores práticos na seleção de algoritmos. A tecnologia predominantemente utilizada neste trabalho é a linguagem Python e, portanto, a biblioteca scikit-learn é uma escolha natural por ser a principal ferramenta de aprendizagem de máquina desta linguagem na última década e possuir um conjunto relevante de algoritmos de agrupamento. (64) As implementações de alguns dos principais algoritmos de agrupamento de dados estão disponíveis nesta biblioteca, como por exemplo: K-médias (65); Propagação de Afinidade (66); Agrupamento Espectral (67); Agrupamento hierárquico utilizando método da mínima variância (68); DBSCAN (Density-Based Spatial Clustering of Applications with Noise) (69); OPTICS (Ordering Points To Identify the Clustering Structure) (70); BIRCH (Balanced Iterative Reducing and Clustering using Hierarchies) (71) e Agrupamento por ajuste de Misturas Gaussianas (72).

\subsubsection{Agrupamento de linhas espectrais estimadas pelo KBDM}

Seja $f: \mathbb{C}^{N} \rightarrow \mathbb{R}^{J \times 4}$ uma função que mapeia o sinal $\widetilde{C}$ no espaço de parâmetros estimados pelo $\mathrm{KBDM}$ com $J=j, M=m$ e $p=1$, tal que

$$
\begin{gathered}
f(\widetilde{C}, j, m, p=1)=\left\{\left(a_{\kappa}^{j, m}, \varphi_{\kappa}^{j, m}, \omega_{\kappa}^{j, m}, \gamma_{\kappa}^{j, m}\right)\right\}=\left\{\Gamma_{\kappa}^{j, m}\right\}=\Gamma^{j, m} \\
\Gamma_{\kappa}^{j, m} \equiv\left(a_{\kappa}^{j, m}, \varphi_{\kappa}^{j, m}, \omega_{\kappa}^{j, m}, \gamma_{\kappa}^{j, m}\right) \in \mathbb{R}^{4} \\
\Gamma^{j, m} \equiv\left\{\Gamma_{\kappa}^{j, m}\right\} \in \mathbb{R}^{j \times 4} \\
\kappa=0,1, \ldots, j-1
\end{gathered}
$$

Se $j=K, m=N / 2$ e o sinal de entrada é exatamente descrito pela Equação 2.1, então, conforme mostrado na seção 2.3 ,

$$
\widetilde{C}=\sum_{\kappa=0}^{K-1} c\left(\Gamma_{\kappa}^{j, m}\right)
$$


onde

$$
c\left(\Gamma_{\kappa}^{j, m}\right)=c_{\kappa}=\tilde{a}_{\kappa} e^{i \widetilde{\Omega}_{\kappa} \tau}
$$

Seja $M_{W} \equiv\left\{m_{0}, \ldots, m_{W-1}\right\}$ o conjunto de valores do parâmetro $M$ no processo de amostragem das estimativas das linhas espectrais realizadas através do KBDM para um sinal $\widetilde{C}$ perturbado. Por simplicidade, a única fonte de perturbação considerada é a presença de ruído branco, independente do tempo e sem correlação entre os canais reais e imaginários e que segue a distribuição $\mathcal{N}(0, \sigma)$. Além disso, apenas um valor fixo $j=J$ será utilizado e seu índice será omitido daqui em diante $\left(\Gamma^{m} \equiv \Gamma^{j=J, m}\right)$.

O conjunto de todas as linhas obtidas neste processo pode ser obtido através da expressão

$$
\Gamma=\bigcup_{m \in M_{W}} \Gamma^{m}
$$

Este conjunto pode ser dividido em duas contribuições

$$
\Gamma=\Gamma^{g} \cup \Gamma^{e}
$$

onde $\Gamma^{g}$ e $\Gamma^{e}$ representam, respectivamente, as linhas genuínas e espúrias estimadas pelo KBDM.

Seja $\Pi^{g}$ o seguinte esquema de particionamento

$$
\begin{gathered}
\Pi^{g}=\Pi\left(\Gamma^{g}\right)=\left\{\Pi_{0}, \Pi_{1}, \ldots, \Pi_{P-1}\right\} \\
\Pi_{i} \cap \Pi_{q}=\emptyset, i \neq q \\
\Gamma^{g}=\bigcup_{i=0}^{P-1} \Pi_{i}
\end{gathered}
$$

onde as linhas contidas em $\Pi_{i}$ são similares entre si.

Seja $\bar{\Pi}_{i}$ o centroide da partição $\Pi_{i}$, tal que

$$
\bar{\Pi}_{i}=\frac{1}{N_{i}} \sum_{i=0}^{N_{i}} \Gamma_{i}=\left(\bar{a}_{i}, \bar{\varphi}_{i}, \bar{\omega}_{i}, \bar{\gamma}_{i}\right)
$$

onde $N_{i}$ é o número de objetos nesta partição e $\left(\bar{a}_{i}, \bar{\varphi}_{i}, \bar{\omega}_{i}, \bar{\gamma}_{i}\right)$ a tupla que representa o valor médio de cada parâmetro das linhas contidas em $\Pi_{i}$.

O componente estimado a partir deste centroide é dado por

$$
\widetilde{c}_{n}^{\prime}\left(\bar{\Pi}_{i}\right)=\bar{a}_{i} e^{-n \bar{\gamma}_{i} \tau} e^{i\left(n \bar{\omega}_{i} \tau+\bar{\varphi}_{i}\right)}
$$


E, portanto, a estimativa final para a reconstrução do sinal é

$$
\widetilde{C}_{n}^{\prime}\left(\Pi^{g}\right)=\sum_{i=0}^{P-1} \widetilde{c}_{n}^{\prime}\left(\bar{\Pi}_{i}\right)=\sum_{i=0}^{P-1} \bar{a}_{i} e^{-n \bar{\gamma}_{i} \tau} e^{i\left(n \bar{\omega}_{i} \tau+\bar{\varphi}_{i}\right)}
$$

O particionamento ótimo para o problema pode ser encontrado através da minimização do resíduo entre o sinal estimado e o sinal de entrada em conjunto ao do uso de medidas de avaliação da qualidade dos agrupamentos. (60) Como o processo de quantificação é realizado na contribuição real no domínio da frequência, o resíduo a ser minimizado por ser estimado através da expressão

$$
\underset{\Pi^{g}}{\arg \min } \frac{1}{2} \sum_{n=0}^{N-1}\left(\operatorname{Re}\left\{\widetilde{S}_{n}^{\prime}\left(\Pi^{g}\right)-\widetilde{S}_{n}\right\}\right)^{2}
$$

De maneira geral, os algoritmos de agrupamento dependem dos valores de um conjunto de parâmetros internos. Estes parâmetros, denominados hiper-parâmetros, são utilizados para adequar as partições resultantes às estruturas presentes em cada conjunto de dados. Portanto o particionamento $\Pi^{g}$ pode ser escrito de maneira genérica através da dependência explícita destes valores, tal que

$$
\Pi^{g}=\Pi^{g}\left(p_{1}, p_{2}, \ldots, p_{N_{H}}\right)
$$

onde $N_{H}$ é o número total de hiper-parâmetros do algoritmo de agrupamento.

O problema consiste agora em encontrar os valores ótimos para os hiper-parâmetros $p_{1}, p_{2}, \ldots, p_{H}$ que minimizam a função residual proposta no problema de otimização dado pela Equação 3.28, resultando em

$$
\begin{gathered}
\underset{p_{1}, p_{2}, \ldots, p_{N_{H}}}{\arg \min } f_{o b j}\left(p_{1}, p_{2}, \ldots, p_{N_{H}}\right) \\
\underset{p_{1}, p_{2}, \ldots, p_{N_{H}}}{\arg \min } \\
\frac{1}{N} \sum_{n=0}^{N-1}\left(\operatorname{Re}\left\{\widetilde{S}_{n}^{\prime}\left(\Pi^{g}\left(p_{1}, p_{2}, \ldots, p_{N_{H}}\right)\right)-\widetilde{S}_{n}\right\}\right)^{2}
\end{gathered}
$$

A extração das linhas genuínas $\Gamma^{g}$ a partir do conjunto completo $\Gamma$ não é um processo trivial. (21) No entanto, alguns algoritmos de agrupamento de dados tem a capacidade de filtrar objetos que são considerados ruídos. Isto é, alguns objetos podem não ser associados a nenhum cluster por não satisfazerem aos critérios de agrupamento utilizados na concepção do algoritmo. Sendo assim, essa família de métodos são naturalmente atrativas para a solução do problema de agrupamento de linhas espectrais estimadas pelo KBDM. De qualquer forma, critérios adicionais de filtragem podem ser previamente utilizados para eliminar linhas sem significado físico, como é o caso de linhas estimadas com taxas de relaxação negativas $\left(\gamma_{k}<0\right)$ ou componentes de baixa amplitude $\left(a_{k} \approx 0\right)$. Embora esta pré-filtragem possa melhorar 0 desempenho dos algoritmos, alguns critérios podem ser subjetivos e dependentes do tipo do 


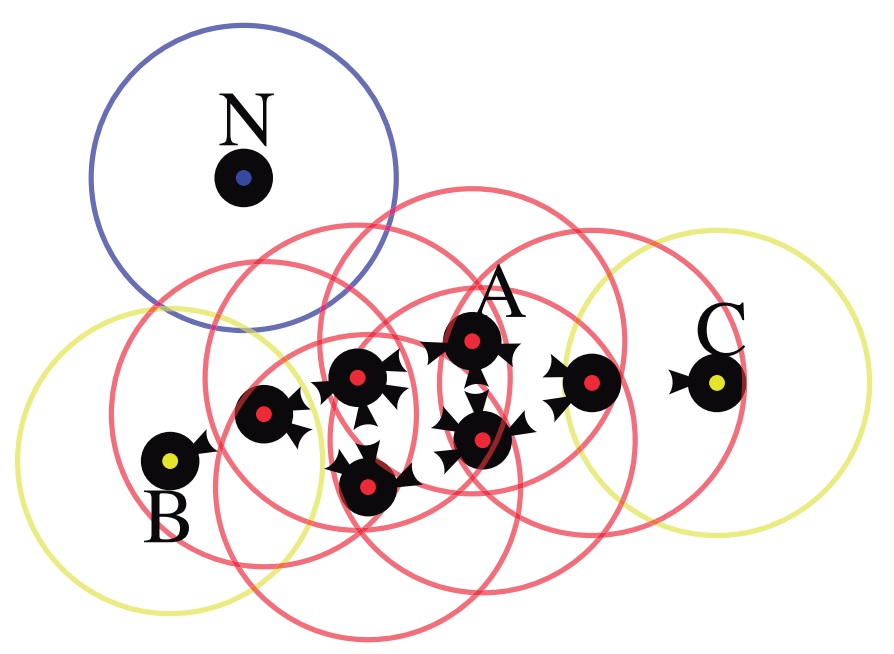

Figura 3.11 - Conjunto de dados bidimensional ilustrando os critérios de agrupamento do algoritmo DBSCAN. Para o agrupamento mostrado, $N_{\min }=4$ e $\epsilon_{\min }$ é representado pelos círculos ao redor de cada amostra. Os pontos vermelhos (incluindo o ponto $A$ ) pertencem ao núcleo do cluster; os pontos amarelos $(B$ e $C)$, embora não pertencentes ao núcleo, também fazem parte do cluster; e apenas o ponto $N$ (em azul) não faz parte do agrupamento.

Fonte: Wikipédia (73).

sinal a ser processado.

\subsubsection{DBSCAN}

O Density-based spatial clustering of applications with noise (DBSCAN) é um algoritmo particional baseado em densidade de pontos. (69) De acordo com critério de agrupamento deste método, a classificação de cada objeto em relação a um cluster se dá através das seguintes premissas:

- Um objeto pertence ao núcleo de um cluster se $N_{\text {min }}$ objetos deste mesmo cluster, incluindo a si mesmo, estiverem a uma distância inferior a $\epsilon_{\min }$;

- Um objeto pertence a um cluster se estiver a uma distância inferior a $\epsilon_{\min }$ de pelo menos um membro do seu respectivo núcleo;

- Um objeto não pertence a nenhum cluster se não se encaixar em nenhum dos dois critérios anteriores.

A Figura 3.11 ilustra a classificação de cada objeto em um conjunto de dados bidimensional 
contendo um único cluster. Neste exemplo, $N_{\min }=4$ e $\epsilon_{\min }$ é representado pelos círculos ao redor de cada objeto. O ponto $A$ é um membro do núcleo do cluster, pois existem quatro membros do núcleo, incluindo a si mesmo, a uma distância inferior a $\epsilon_{\min }$. A mesma classificação é válida aos demais pontos demarcados pela cor vermelha. Os pontos $B$ e $C$ não são membros do núcleo, mas pertencem ao cluster por estarem a uma distância inferior a $\epsilon_{\min }$ de objetos pertencentes ao núcleo. O ponto $N$ não se enquadra nos critérios de agrupamento e portanto não pertence ao cluster.

Para demonstrar a aplicabilidade da formulação do agrupamentos das linhas espectrais estimadas pelo KBDM apresentado na seção 3.3.2, o algoritmo DBSCAN foi utilizado para encontrar automaticamente as linhas espectrais médias a partir do sinal simulado através dos parâmetros descritos pela Tabela 2.1 com adição de ruído gaussiano $(\sigma=0.02)$ e $N=2048$. O conjunto $\Gamma$ foi produzido através de 20 execuções do KBDM com $M=200,210,220, \ldots, 390$ e $J=30$.

Como a distância euclidiana foi adotada, para que nenhum parâmetro do sinal prevaleça perante aos demais em função da heterogeneidade das escalas, os atributos foram normalizados, de maneira que

$$
x_{\mathrm{norm}}^{j}=\frac{x_{i}^{j}-x_{\max }^{j}}{x_{\max }^{j}-x_{\min }^{j}}
$$

onde $x_{i}^{j} \in\left\{a_{i}, \varphi_{i}, \omega_{i}, \gamma_{i}\right\}$.

Esta transformação fornece a garantia de que o valor de cada atributo estará no intervalo $[0,1]$. É importante ressaltar que o processo de normalização se dá apenas no contexto do agrupamento dos objetos que representam as linhas espectrais. O cálculo dos valores médios dos parâmetros é feito no espaço original dos atributos.

A Figura 3.12 fornece a visualização bidimensional dos valores da função objetiva adotada neste exemplo. O domínio de busca é representado através dos eixos vertical e horizontal que se referem, respectivamente, aos valores dos parâmetros $\epsilon_{\min }$ e $N_{\min }$ do DBSCAN. As regiões escuras são as que representam maior inadequação dos parâmetros, enquanto as mais claras estão associadas a menores diferenças entre o espectro estimado e o espectro do sinal de entrada. Para fornecer um detalhamento visual melhor, o logaritmo da função objetiva sugerida anteriormente foi utilizado, de modo que

$$
f_{\text {obj }}\left(N_{\text {min }}, \epsilon_{\min }\right)=\log \left(\frac{1}{N} \sum_{n=0}^{N-1}\left[\operatorname{Re}\left\{\widetilde{S}_{n}^{\prime}\left(\Pi\left(N_{\min }, \epsilon_{\min }\right)\right)-\widetilde{S}_{n}\right\}\right]^{2}\right)
$$

onde $\Pi$ é o particionamento realizado a partir de todas as linhas estimadas pelo KBDM, uma vez que o DBSCAN é capaz de lidar com a presença das linhas espúrias internamente. 


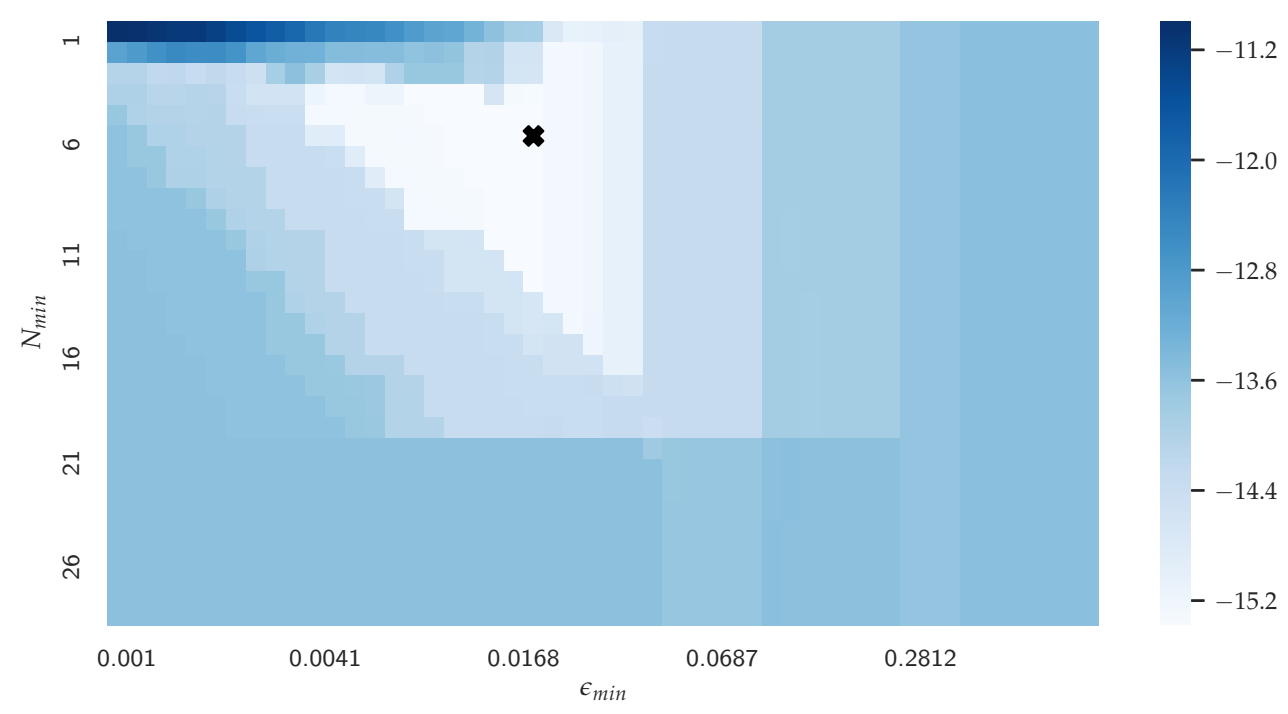

Figura 3.12 - Função objetiva, $f_{o b j}\left(N_{\min }, \epsilon_{\min }\right)$, para otimização dos parâmetros $\epsilon_{\min }$ e $N_{\min }$ do algoritmo DBSCAN na busca por linhas espectrais médias através de um conjunto de estimativas do KBDM feitas em múltiplos truncamentos do sinal. As regiões de maior erro são as mais escuras, enquanto as mais claras representam menores diferenças residuais entre o sinal estimado e o sinal de entrada. $O$ marcador $X$ em preto denota o ponto mínimo global dentro da região de busca $\left(\epsilon_{\min } \approx 0.0193 \mathrm{e}\right.$ $\left.N_{\min }=6\right)$.

Fonte: Elaborada pelo autor.

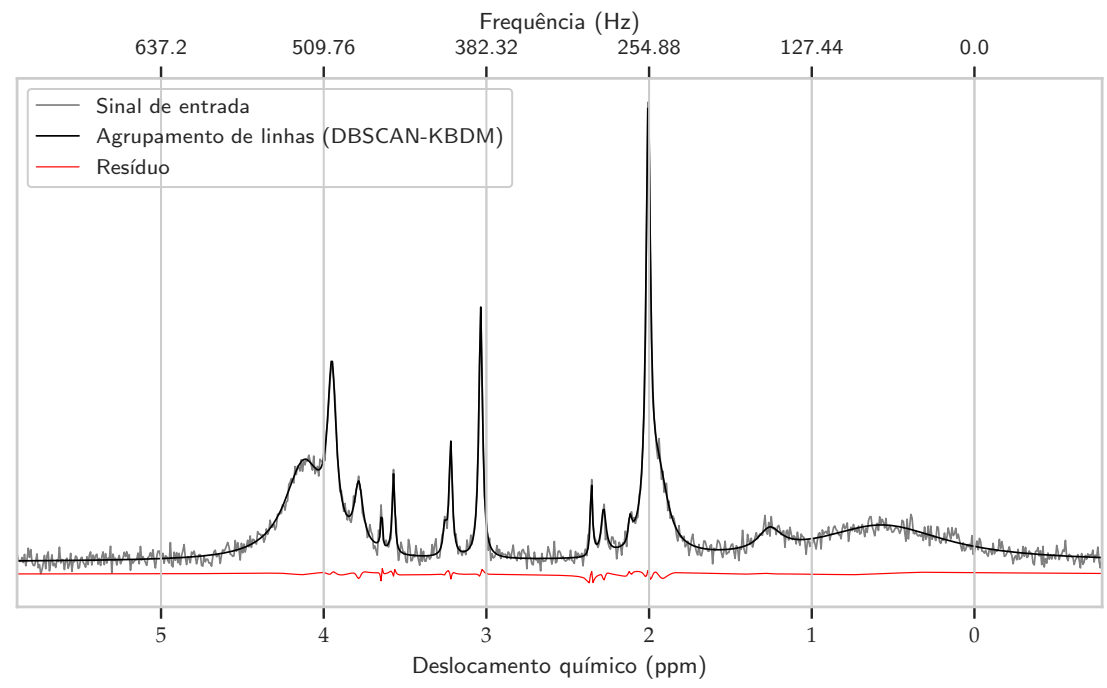

Figura 3.13 - Demonstração do ajuste do sinal sintético descrito pela Tabela 2.1 com adição de ruído gaussiano $(\sigma=0.02)$ através do agrupamento de linhas espectrais estimadas pelo KBDM utilizando o algoritmo DBSCAN. Os utilizados pelo DBSCAN $\left(\epsilon_{\min } \approx 0.0193\right.$ e $N_{\min }=6$ ) foram obtidos através do processo de otimização demonstrado na $\mathrm{Fi}$ gura 3.12 .

Fonte: Elaborada pelo autor. 
Tabela 3.1 - Parâmetros das linhas obtidas através de múltiplas amostragens do KBDM com agrupamento de linhas através do DBSCAN para o exemplo mostrado na Figura 3.13.

\begin{tabular}{rrrrr}
\hline & $a_{k}$ & $\gamma_{k}^{-1}(\mathrm{~s})$ & $f_{k}(\mathrm{~Hz})$ & $\varphi_{k}\left(^{\circ}\right)$ \\
\hline 0 & 1.027808 & 0.002617 & 72.257001 & -0.098064 \\
1 & 0.112545 & 0.014482 & 160.557293 & -3.306097 \\
2 & 0.340798 & 0.017740 & 247.769349 & -2.187043 \\
3 & 0.401965 & 0.077329 & 255.514020 & 0.907654 \\
4 & 0.019195 & 0.093176 & 270.067193 & -18.040024 \\
5 & 0.047380 & 0.071678 & 290.589249 & -5.205845 \\
6 & 0.035876 & 0.156706 & 300.155743 & -6.281410 \\
7 & 0.205814 & 0.091514 & 386.742400 & 0.123158 \\
8 & 0.080830 & 0.104930 & 410.240348 & 0.453097 \\
9 & 0.020348 & 0.099058 & 414.922980 & -2.096786 \\
10 & 0.045200 & 0.131662 & 454.983454 & 3.736631 \\
11 & 0.014651 & 0.214282 & 464.305373 & 6.585416 \\
12 & 0.113052 & 0.040074 & 482.364723 & -0.512785 \\
13 & 0.299099 & 0.040242 & 503.247630 & 2.343672 \\
14 & 0.824636 & 0.008632 & 525.285504 & 0.179561 \\
\hline
\end{tabular}

Fonte: Elaborada pelo autor.

Para este estudo realizado, o melhor agrupamento se dá quando $\epsilon_{\min } \approx 0.0193$ e $N_{\min }=6$.

O significado do parâmetro $\epsilon_{\min }$ é de difícil interpretação, principalmente no espaço de atributos normalizado. No entanto, é possível afirmar que, quanto menor seu valor, maior a compactação dos clusters estimados.

Por outro lado, o parâmetro $N_{\text {min }}$ possui interpretação mais simples por estar diretamente ligado à quantidade mínima de elementos em cada cluster. Sua interpretação está, portanto, relacionada ao que foi mostrado na Figura 3.10 sobre a taxa de reconstrução bem sucedida para cada linha do sinal na presença de ruído. Como algumas linhas possuem menor taxa de reconstrução, os respectivos clusters possuem menos elementos, justificando a razão pela qual o número ótimo obtido $\left(N_{\min }=6\right)$ é inferior ao número de execuções do KBDM no processo de amostragem.

A Figura 3.13 mostra o espectro estimado para os parâmetros apresentados na Tabela 3.1. A linha que representada a diferença residual entre o sinal estimado e o sinal verdadeiro é mostrada em vermelho. Pequenas diferenças existem apenas nas regiões de frequência de maior densidade de picos.

As Figuras 3.14-3.16 mostram os diagramas de dispersão entre as amplitudes, taxas de re- 
laxação e frequências estimadas para o exemplo apresentado. Os pontos pretos simbolizam os valores obtidos para os parâmetros de cada linha estimada pelo KBDM no processo de amostragem. Os marcadores $\mathbf{X}$ denotam os centroides dos clusters, que representam as estimativas finais utilizando o método de agrupamento através do DBSCAN. Para fins de comparação, os marcadores pretos + representam os valores verdadeiros (Tabela 2.1).

Através destes diagramas é possível observar a tendência de formação de clusters majoritariamente constituídos de linhas genuínas, enquanto as linhas espúrias tendem a se espalhar. Este fenômeno pode ser mais facilmente observado no plano $\gamma_{k}^{-1}-a_{k}$ do espaço de atributos (Figura 3.14).

Ao menos duas desvantagens imediatas do DBSCAN no agrupamento de linhas espectrais estimadas pelo KBDM podem ser citadas. A primeira delas é a necessidade do ajuste simultâneo dos parâmetros $N_{\min }, \epsilon_{\min }$ na minimização do erro global do ajuste, representado pela função objetiva $f_{o b j}\left(N_{\min }, \epsilon_{\min }\right)$. No exemplo mostrado, estes parâmetros foram obtidos através de otimização por força bruta em todos os valores possíveis contidos na grade bidimensional ilustrada na Figura 3.12. Embora esta abordagem tenha sido suficiente para demonstrar alguns aspectos importantes do problema, ela não representa a maneira mais eficiente de executar esta tarefa. Além disso, a resolução da grade, especialmente na dimensão que representa $\epsilon_{\min }$, pode ser impactante na obtenção dos resultados ótimos.

É possível observar através das Figuras 3.14-3.16 que o espalhamento das estimativas em torno do valor verdadeiro de cada linha do mesmo sinal pode ocorrer de maneira heterogênea. Alguns fatores associados a este fenômeno são a relação sinal-ruído de cada pico e o grau de sobreposição espectral entre eles. Portanto, a segunda limitação deste método, que é ainda mais relevante, consiste no fato de que os parâmetros $\epsilon_{\min }$ e $N_{\min }$, que definem a densidade dos clusters, são ajustados de maneira global. Isto é, clusters com diferentes densidades de pontos não podem ser ajustados simultaneamente.

Como a heterogeneidade estrutural dos clusters tende a aumentar com o nível de ruído no sinal, o particionamento resultante do processo de otimização dos parâmetros $\epsilon_{\min }$ e $N_{\min }$ poderá possuir um ou mais clusters contendo linhas referentes à componentes distintas do sinal. Embora a análise de cada cluster possa ser realizada posteriormente, a complexidade do algoritmo e o risco de soluções sem significado físico aumentam significativamente nestes casos.

Durante a realização deste trabalho, foi proposto em (74) uma solução inicial para este problema através do ajuste iterativo do sinal. É assumido um valor fixo para $N_{\min }$, geralmente da ordem da metade do número de amostras do $\operatorname{KBDM}\left(N_{\min }=W / 2\right)$. Atribui-se $\epsilon_{\min }=\epsilon_{0}$, 


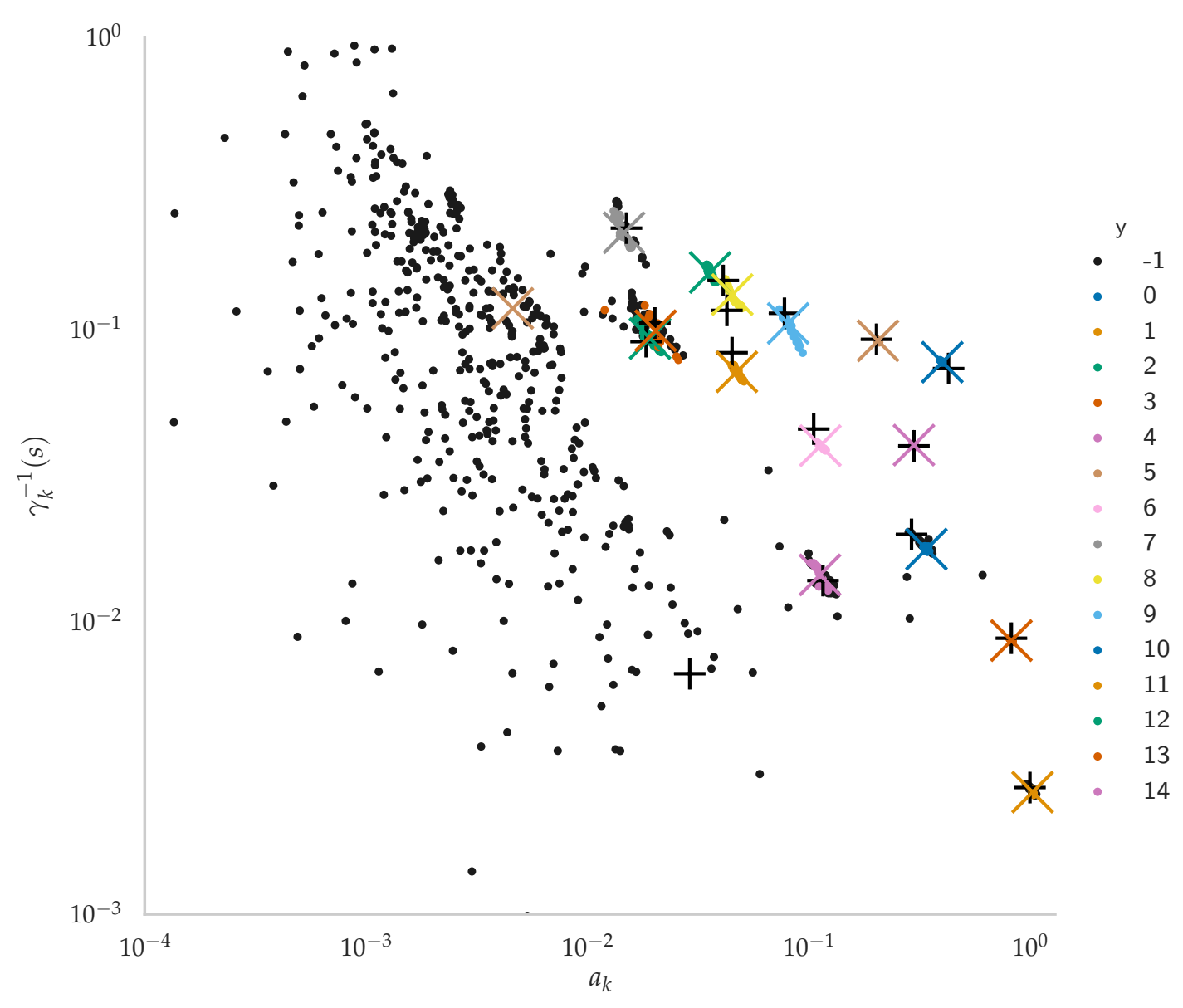

Figura 3.14 - Diagrama de dispersão para os tempos de relaxação transversal $\left(\gamma_{k}^{-1}\right)$ e as amplitudes $a_{k}$ das linhas estimadas para o sinal contendo ruído mostrado na Figura 3.13. Os pequenos círculos representam as múltiplas estimativas obtidas através do processo de amostragem do KBDM com $M=200,210,220, \ldots, 390$ e $J=30$. Os círculos pretos são os pontos filtrados pelo DBSCAN, enquanto os círculos coloridos representam as linhas de um mesmo cluster. Os marcadores $\mathbf{X}$ simbolizam os centroides de cada cluster, utilizados como estimativa final do método para cada linha espectral. Os marcadores + representam as linhas verdadeiras apresentadas na Tabela 2.1. Enquanto as estimativas genuínas tendem a formar aglomerados, as linhas espúrias possuem alta dispersão. Ao todo, 16 linhas foram estimadas. Uma delas, no entanto, de caráter espúrio. Em função do nível de ruído, o pico \#5 não foi detectado.

Fonte: Elaborada pelo autor. 


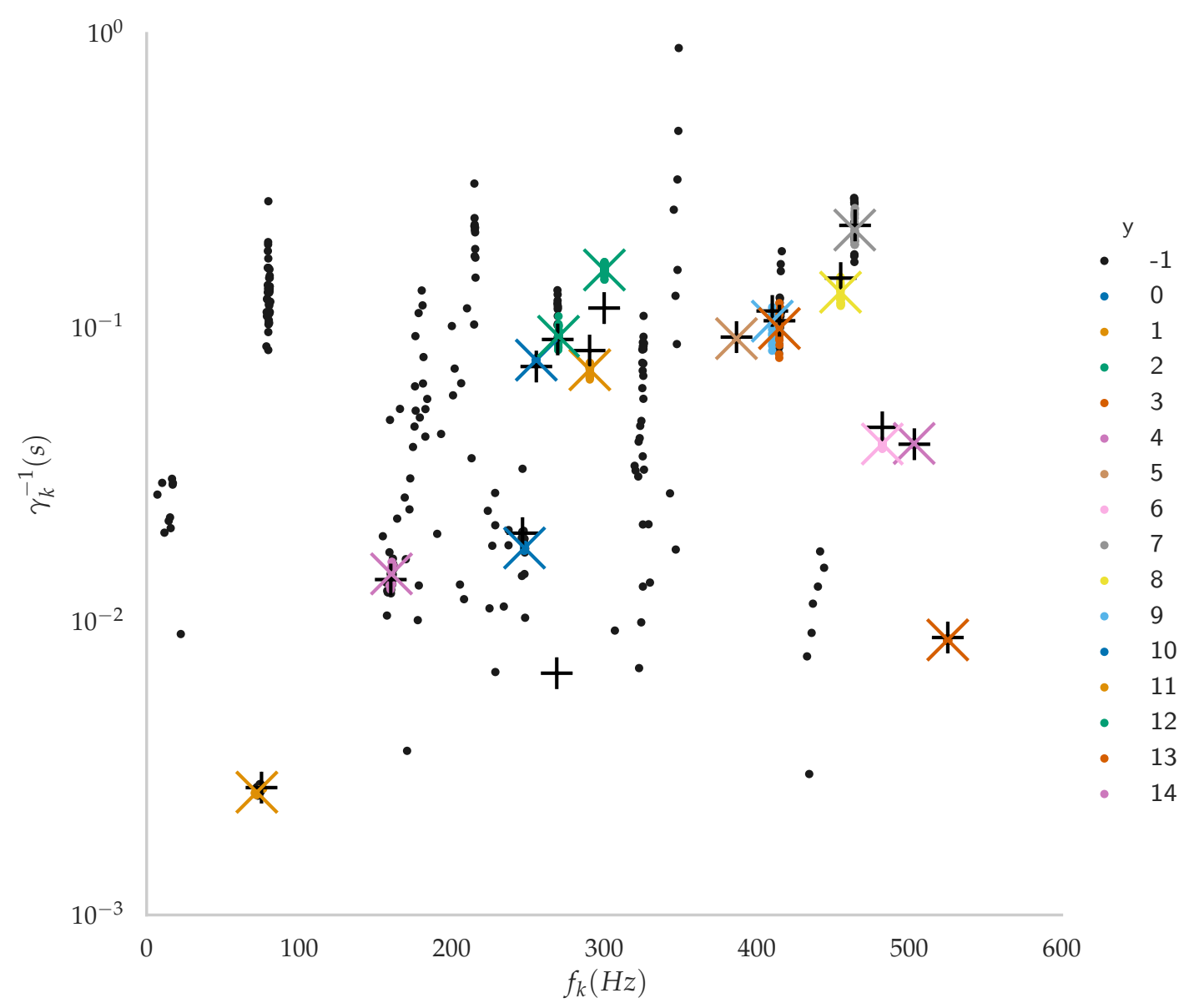

Figura 3.15 - Diagrama de dispersão para os tempos de relaxação transversal $\left(\gamma_{k}^{-1}\right)$ e as frequências $f_{k}$ das linhas espectrais estimadas. Os pequenos círculos representam as múltiplas estimativas obtidas através do processo de amostragem do KBDM com $M=200,210,220, \ldots, 390$ e $J=30$. Os círculos pretos são os pontos filtrados pelo DBSCAN, enquanto os círculos coloridos representam as linhas de um mesmo cluster. Os marcadores $\mathbf{X}$ simbolizam os centroides de cada cluster, utilizados como estimativa final do método para cada linha espectral. Os marcadores + representam as linhas verdadeiras apresentadas na Tabela 2.1. Ao todo, 16 linhas foram estimadas. Uma delas, no entanto, de caráter espúrio. Em função do nível de ruído, o pico \#5 não foi detectado.

Fonte: Elaborada pelo autor.

sendo $\epsilon_{0}$ um valor arbitrariamente pequeno. O DBSCAN é aplicado no espaço de dados normalizado com estes parâmetros.

Se existirem clusters válidos, um sinal parcial é construído através do parâmetros médios destes. Os clusters inválidos são descartados. Este sinal parcial é subtraído do sinal entrada, resultando em um sinal residual. Se existirem apenas clusters inválidos, $\epsilon_{\min }$ é reduzido até que se encontre algum cluster válido. Caso nenhum cluster seja detectado, $\epsilon_{\min }$ é aumentado. Se o espaço estiver corretamente normalizado, quando $\epsilon_{\min }$ excede a unidade, 0 algoritmo deve ser finalizado. 


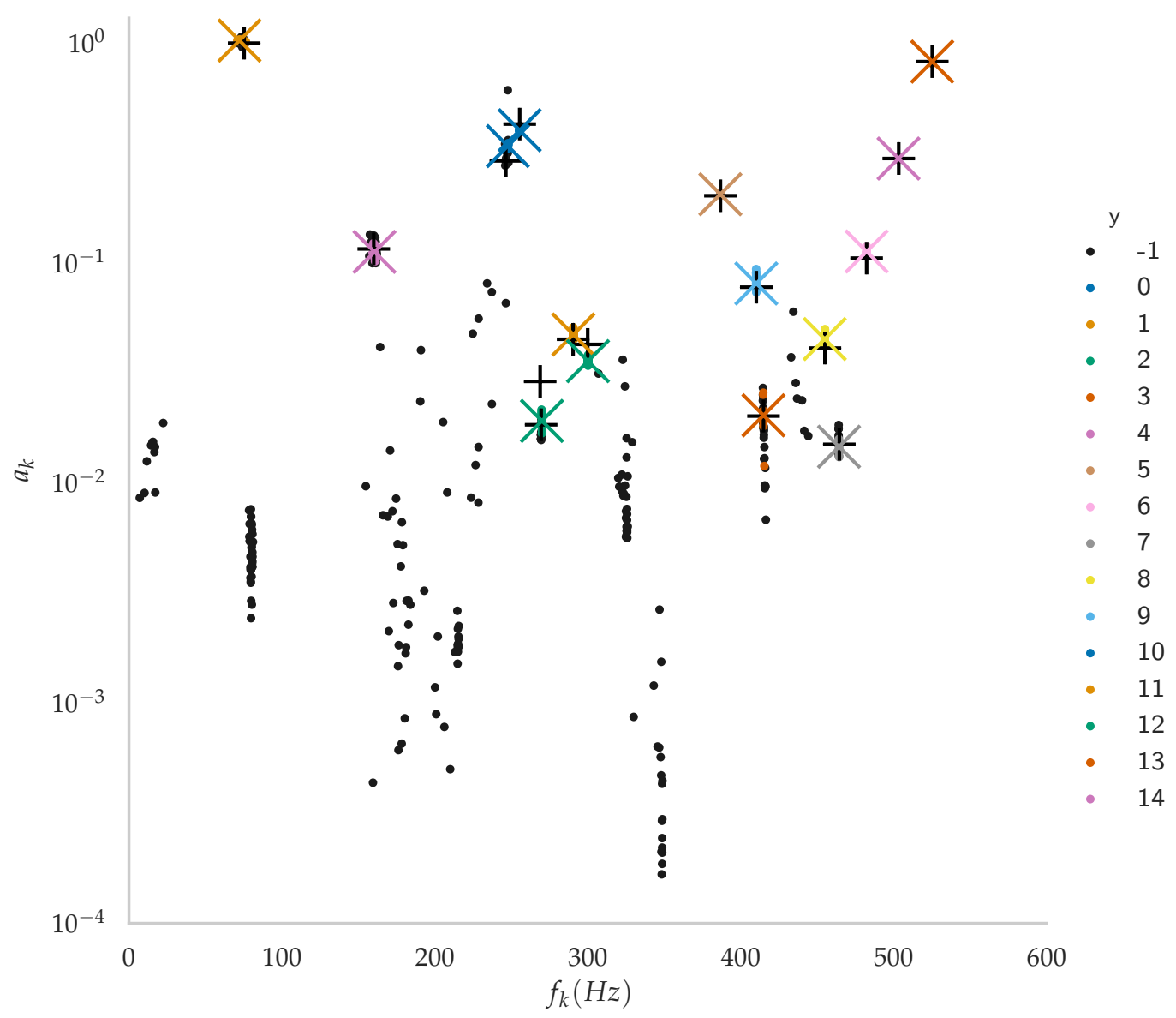

Figura 3.16 - Diagrama de dispersão para as amplitudes $a_{k}$ e as frequências $f_{k}$ das linhas espectrais estimadas. Os pequenos círculos representam as múltiplas estimativas obtidas através do processo de amostragem do KBDM com $M=200,210,220, \ldots, 390$ e $J=30$. Os círculos pretos são os pontos filtrados pelo DBSCAN, enquanto os círculos coloridos representam as linhas de um mesmo cluster. Os marcadores $\mathbf{X}$ simbolizam os centroides de cada cluster, utilizados como estimativa final do método para cada linha espectral. Os marcadores + representam as linhas verdadeiras apresentadas na Tabela 2.1. Ao todo, 16 linhas foram estimadas. Uma delas, no entanto, de caráter espúrio. Em função do nível de ruído, o pico \#5 não foi detectado.

Fonte: elaborada pelo autor.

Fonte: Elaborada pelo autor.

No sinal residual, as amostragens do KBDM são realizadas novamente. O DBSCAN é aplicado novamente e a etapa anterior se repete até que o sinal residual contenha apenas ruído ou não seja possível gerar mais clusters válidos.

O critério de qualidade para invalidar um cluster pode ser baseado em heurísticas simplificadas, como a verificação do número de elementos do cluster em relação ao número de amostras do KBDM, por exemplo. Outras medidas de qualidade do cluster, como a silhueta, também podem ser utilizadas (75). 
Embora este algoritmo seja funcional em muitos casos, a escolha de $\epsilon_{0}$ e $\circ$ passo em que $\epsilon_{\min }$ é aumentado ou reduzido durante as varreduras dependem do espectro analisado. A sobreposição dos picos e as diferenças das ordens de magnitudes entre os parâmetros de cada componente são fatores que impactam essas decisões. Isto é, na prática, se consegue bons resultados para problemas distintos apenas através de ajustes manuais para estes parâmetros, o que definitivamente não é adequado para solução do problema.

\subsubsection{HDBSCAN}

As restrições do DBSCAN mencionadas na seção anterior não são consequências particulares do problema do agrupamento de linhas espectrais. De fato, estas mesmas limitações foram utilizadas como motivação no desenvolvimento de uma versão hierárquica para o DBSCAN, o HDBSCAN. (76-77)

Um dos objetivos do HDBSCAN é permitir a visualização hierárquica de todas as possíveis combinações de clusters gerados pelo DBSCAN* simultaneamente. Esta tarefa pode ser realizada a partir da criação de dendrogramas e uma solução particular entre os possíveis agrupamentos pode ser obtida através do corte em uma dada altura da árvore que representa essa estrutura.

Para atingir este objetivo, os autores formularam o HDBSCAN através da construção de uma estrutura denominada Grafo de Alcance Mútuo, $G_{\mathrm{am}}$, um grafo completo onde cada objeto do conjunto de dados é um nó e o peso de cada aresta representa a distância de alcance mútuo entre os objetos, definida por

$$
d_{\mathrm{am}}\left(x_{p}, x_{q}\right)=\max \left\{d_{\text {núcleo }}\left(x_{p}\right), d_{\text {núcleo }}\left(x_{q}\right), d\left(x_{p}, x_{q}\right)\right\}
$$

onde $d_{\text {núcleo }}\left(x_{p}\right)$ e $d_{\text {núcleo }}\left(x_{q}\right)$ são as distâncias dos objetos $x_{p}$ e $x_{q}$ até seus $N_{\text {min }}$-ésimos respectivos vizinhos; e $d\left(x_{p}, x_{q}\right)$ a distância euclidiana entre $x_{p}$ e $x_{q}$. Portanto, o único hiperparâmetro do HDBSCAN é $N_{\text {min }}$.

O próximo passo consiste na criação de um árvore geradora mínima a partir do grafo $G_{\mathrm{am}}$. Este processo pode ser realizado através do algoritmo de Prim. (78-79) Conforme mostrado em (80), a árvore geradora mínima possui toda a informação necessária para a criação do dendrograma do algoritmo de agrupamento aglomerativo utilizando ligação simples. Isto é, o HDBSCAN equivale a este outro algoritmo no espaço transformado das distâncias de alcance 
mútuo, mais precisamente alinhado com a abordagem apresentada em (81).

Embora estas contribuições já tenham sua importância, os autores foram além e propuseram, no mesmo trabalho, uma maneira de encontrar o particionamento ótimo dos dados através da definição de uma medida de estabilidade dos clusters, denominada excesso de massa. A solução do problema da otimização do excesso de massa permite que o corte em cada ramificação da árvore seja feito em alturas distintas. Deste modo, o algoritmo viabiliza a detecção de clusters com densidades distintas, indo ao encontro das necessidades do problema de agrupamento de linhas espectrais. Outro trabalho relacionado ao desenvolvimento de um arcabouço necessário para obtenção ótima de clusters a partir de algoritmos de agrupamento hierárquico também foi publicado por Campello et al. (82)

A difusão e adoção deste novo algoritmo de agrupamento pela comunidade foi bastante influenciada pelo desenvolvimento da biblioteca de código aberto hdbscan. (83) Ela foi publicada no repositório scikit-learn-contrib e, portanto, é compatível com o arcabouço da biblioteca scikit-learn. (84) Além de possibilitar o uso desse algoritmo através da linguagem Python, diversas otimizações computacionais foram realizadas pela hdbscan, como por exemplo, a utilização do algoritmo de Boruvka na criação das árvores geradoras mínimas. (85) Esta implementação será utilizada neste trabalho.

\subsection{Transformações no espaço de dados}

Conforme já discutido, uma linha espectral que caracteriza uma componente Lorentziana do sinal pode ser representada por um ponto no espaço dos seus respectivos parâmetros. A tupla $\left(a_{k}, \gamma_{k}, \omega_{k}, \varphi_{k}\right) \in \mathbb{R}^{4}$ representa univocamente cada linha espectral através de um ponto neste espaço. Além de permitir a reconstrução do sinal, cada um destes quatro parâmetros são carregados de significado físico sobre cada componente. No entanto, a representação destes parâmetros no contexto de agrupamento das linhas estimadas pelo KBDM requer uma característica adicional: a separação adequada entre linhas que pertencem ou não a um mesmo cluster. Esta necessidade surge a partir do uso de medidas de similaridades baseadas em distância euclidiana entre parâmetros que caracterizam as linhas.

Os fenômenos físicos de interesse neste problema, que são de caráter dissipativo, são descritos necessariamente por linhas que possuem taxa de relaxação positiva $\left(\gamma_{k}>0\right)$ e, além disso, para que as características espectrais do sinal sejam preservadas, a amostragem do mesmo deve ser densa o suficiente para obedecer ao Teorema de Nyquist-Shannon de maneira 
que as frequências de cada componente possam obedecer a seguinte restrição: (20)

$$
0<\omega_{k}<\frac{2 \pi}{\tau}
$$

Por meio das Equaç̃ões 2.68-2.70, os parâmetros $\gamma_{k}$ e $\omega_{k}$ estão relacionados aos autovalores $\tilde{\mu}_{k}=e^{i \widetilde{\Omega}_{k} \tau}$ da equação 2.66 .

A Figura 3.17 ilustra a representação deste subespaço autovalores $\tilde{\mu}_{k}$ no plano complexo. A restrição $\gamma_{k}>0$ é representada pela região sombreada no interior do círculo, onde $\left\|\tilde{\mu}_{k}\right\|<1$. Seja o autovalor $\mu_{0}=\mu_{0}^{r e}+i \mu_{0}^{i m}$ representado pelo ponto preto na figura. Os eixos horizontal e vertical representam, respectivamente, suas contribuições reais e imaginárias. $\mathrm{O}$ arco formado entre o eixo horizontal e a linha que vai deste ponto até a origem é dado pelo produto do intervalo de amostragem, $\tau$, pela frequência angular $\omega_{0}$ do componente. A distância deste ponto até a origem é dada por $\left\|\tilde{\mu}_{0}\right\|=e^{-\gamma_{0} \tau}$.

Este subespaço naturalmente se torna, portanto, um candidato a ser investigado com o intuito de se obter separação adequada entre clusters de linhas distintas. Entretanto, a ordem de grandeza típica para os valores de $\gamma_{k}$ impede uma separação radial significativa entre os componentes. A Figura 3.18 mostra o sinal simulado a partir da Tabela 2.1 representado neste plano. O tamanho de cada marcador nesta figura é proporcional à amplitude da respectiva componente.

A componente radial do plano complexo que representa o autovalor $\tilde{\mu}_{k}$ pode ser obtida através da definição dada pela Equação 2.4

$$
\tilde{\mu}_{k}=e^{i \tilde{\Omega}_{k} \tau}=e^{-\gamma_{k} \tau} e^{i \omega_{k} \tau}=r\left(\gamma_{k}\right) e^{i \omega_{k} \tau}
$$

Portanto, a separação radial entre os clusters pode ser ampliada, por exemplo, a partir da transformação

$$
r\left(\gamma_{k}\right)=\ln \left(\gamma_{k}\right)
$$

Por outro lado, como mostrado pela Figura 3.19, essa transformação afeta a separação de garantida pela distribuição entre as frequências.

Algumas informações sobre a natureza dos parâmetros $a_{k}, \gamma_{k}, \omega_{k}$ e $\varphi_{k}$ podem ser exploradas na construção de uma transformação eficiente:

1. Banda espectral de interesse: o intervalo de frequências onde a informação desejada a respeito do espectro processado é normalmente conhecido a priori. Filtrar as linhas 


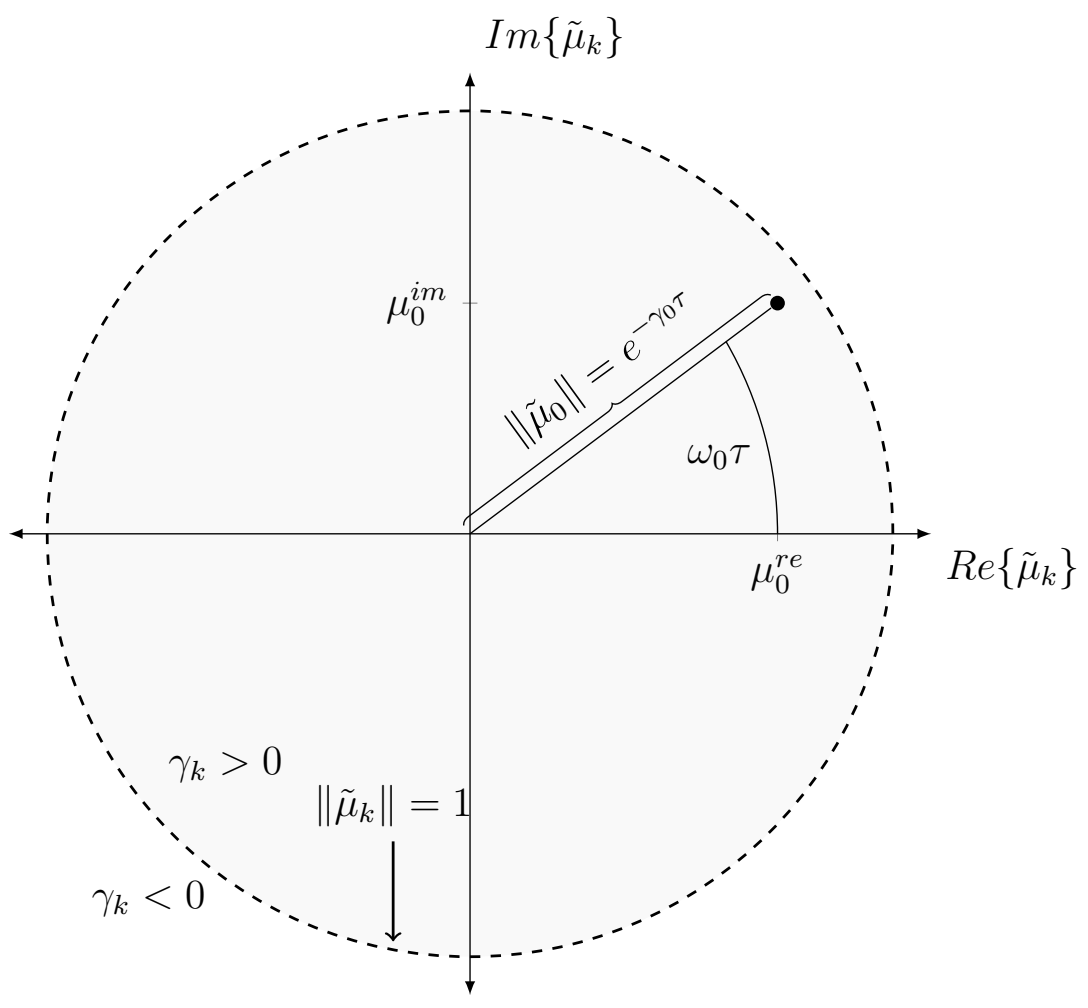

Figura 3.17 - Plano complexo para para os autovalores da equação 2.66.

Fonte: Elaborada pelo autor.

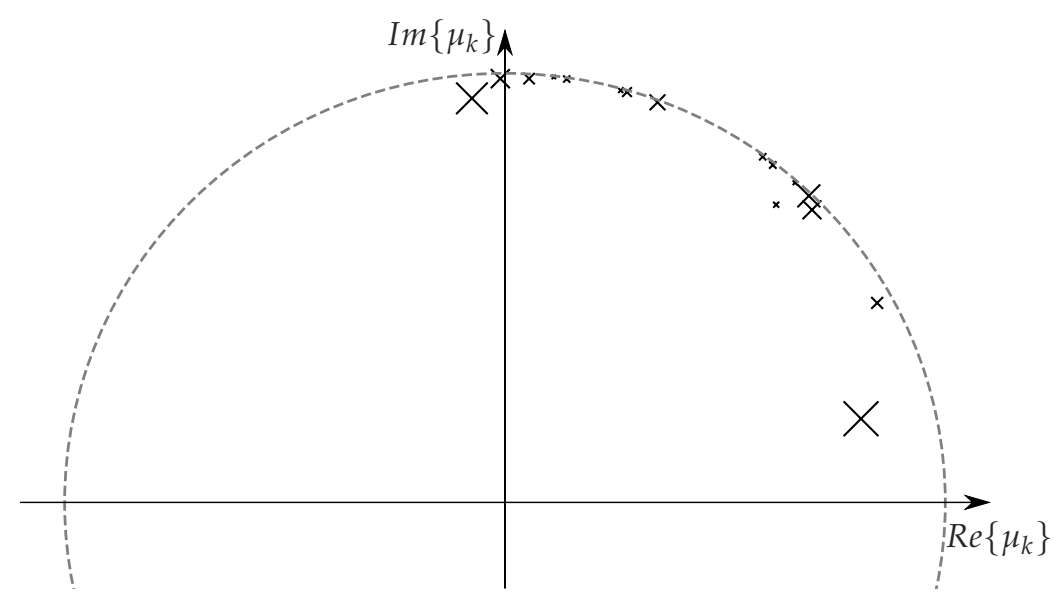

Figura 3.18 - Componentes reais e imaginárias dos autovalores $\tilde{\mu}_{k}=e^{\widetilde{\Omega}_{k} i \tau}$ para os parâmetros do sinal simulado descrito pela Tabela 2.1. Os tamanhos dos marcadores são proporcionais às amplitudes de cada componente.

Fonte: Elaborada pelo autor. 


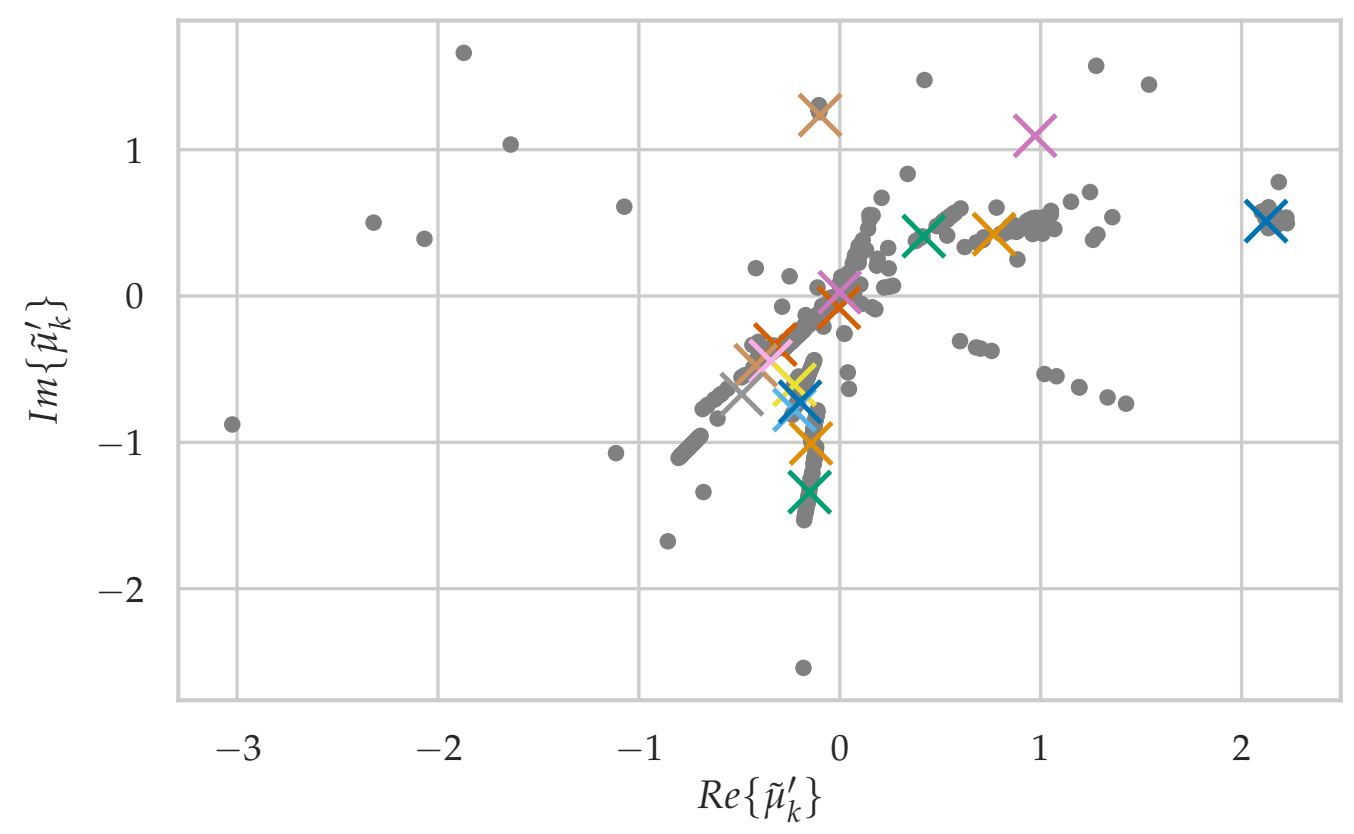

Figura 3.19 - Plano complexo para os autovalores transformados pela Equação 3.37. A separação radial é obtida em detrimento da separação de frequências para as componentes com menores valores de $r\left(\gamma_{k}\right)$. O pontos pretos são componentes amostradas pelo KBDM para um sinal simulado com ruído $(\sigma=0.03)$ a partir dos parâmetros da Tabela 2.1. Os marcadores $\mathbf{X}$ coloridos representam os valores verdadeiros dos parâmetros.

Fonte: Elaborada pelo autor. 
fora região de interesse reduz o tempo computacional no agrupamento de linhas e na reconstrução do sinal e também colabora na realização de transformações por aumentar a eficácia da padronização dos dados. Entretanto, nenhuma hipótese pode ser feita sobre a distribuição das frequências no interior da região de interesse para o caso geral.

2. Distribuição das fases do sinal: os espectros são geralmente processados após a correção de fase do sinal. Neste cenário, todas as estimativas genuínas devem possuir as respectivas fases próximas a zero. No entanto, alguns artefatos como o alargamento de linhas causado por inomogeneidade de campo magnético podem resultar em estimativas de picos pelo KBDM constituídos por múltiplas componentes que possuem fase diferente de zero. Ainda que este tipo linha tenha seu significado físico comprometido, em aplicações onde a supressão de sinais é desejada, esta limitação do método não se torna tão relevante. Por este motivo, não será feita nenhuma hipótese sobre a distribuição das fases no sinal nos algoritmos desenvolvidos neste trabalho, mas a informação é deixada aqui como futura referência para aplicações específicas que possam explorá-la.

3. Distribuição dos valores das taxas de relaxação transversal: idealmente, o sinal processado deve ser amostrado em um intervalo temporal onde o retorno do sistema ao seu estado de equilíbrio tenha sido obtido. Ou seja, os últimos pontos do sinal devem conter apenas as contribuições causadas pelo ruído. Mesmo em aplicações onde o sinal adquirido é truncado, a quantidade de pontos deve ser no mínimo suficiente para obtenção da informação de todos os componentes. Por outro lado, componentes com tempo de relaxação transversal menores do que o intervalo de amostragem $\tau$ não são detectadas. Pode-se assumir, portanto, que as componentes com significado físico estão contidas no intervalo

$$
\tau<\gamma_{k}^{-1}<N \tau
$$

Como não é possível realizar nenhuma hipótese para a distribuição destes valores no o interior deste intervalo, a justificativa para o uso de operações logarítmicas durante a construção de um espaço de separabilidade adequada se dá pelo fato de que a faixa dinâmica desta grandeza é da ordem de

$$
\mathcal{D} \approx N \tau / \tau=N
$$

Isto é, em aplicações típicas, variações da ordem de 3 ou 4 casas decimais podem existir entre os menores e maiores valores de $\gamma_{k}^{-1}$.

4. Distribuição dos valores de amplitude: quando o sinal não possui ruído a separação entre as amplitudes genuínas e espúrias são claras, conforme mostrado no Capítulo 2. 
Na presença de ruído, porém, apenas as maiores amplitudes consideravelmente maiores do que o desvio padrão do ruído podem ser diferenciadas facilmente. No caso geral, este parâmetro pode assumir quaisquer valores e, portanto, a separabilidade dos clusters também pode ser favorecida através do uso de transformações logarítmicas e normalizações adequadas. O corte que define o menor valor estimado possível na presença de ruído, porém, se torna subjetivo e, portanto, não será feita nenhuma hipótese neste sentido no algoritmo desenvolvido.

Com base nesta discussão, as seguintes transformações são propostas na construção do espaço de dados $\mathcal{X} \in \mathbb{R}^{4}$ que representam as linhas espectrais

$$
\begin{gathered}
x_{k}^{1}=\ln \left(a_{k}\right) \\
x_{k}^{2}=\ln \left(\gamma_{k}\right) \\
x_{k}^{3}=\omega_{k} \\
x_{k}^{4}=\varphi_{k}
\end{gathered}
$$

resultando nos vetores

$$
\begin{gathered}
\mathbf{x}_{k}=\left(x_{k}^{1}, x_{k}^{2}, x_{k}^{3}, x_{k}^{4}\right) \\
\mathbf{x}_{k} \in \mathcal{X}
\end{gathered}
$$

Embora muito útil em diversos casos, a normalização apresentada anteriormente pela Equação 3.32 não é eficaz quando os dados possuem outliers causados pela presença de ruído. Estes picos espúrios prejudicam a escala no cálculo das distâncias euclidianas em espaços normalizados. Portanto, um tratamento mais adequado é dado pela padronização dos dados, que leva em consideração a presença destes artefatos.

A transformação que atribui novas coordenadas aos vetores deste espaço de dados por meio do processo de padronização é dada por

$$
\begin{gathered}
x_{k}^{i} \rightarrow \frac{x_{k}^{i}-\bar{x}^{i}}{\sigma^{i}} \\
x_{k}^{i} \in \mathcal{X} \\
i=1,2,3,4
\end{gathered}
$$

onde $\bar{x}_{i}$ e $\sigma^{i}$ são, respectivamente, os valores médio e o desvio padrão de $x_{k}^{i}$.

A Figura 3.20 ilustra o mesmo exemplo mostrado anteriormente pela Figura 3.19 representado pelas transformações descritas pelas Equações 3.40-3.43 e 3.46. 

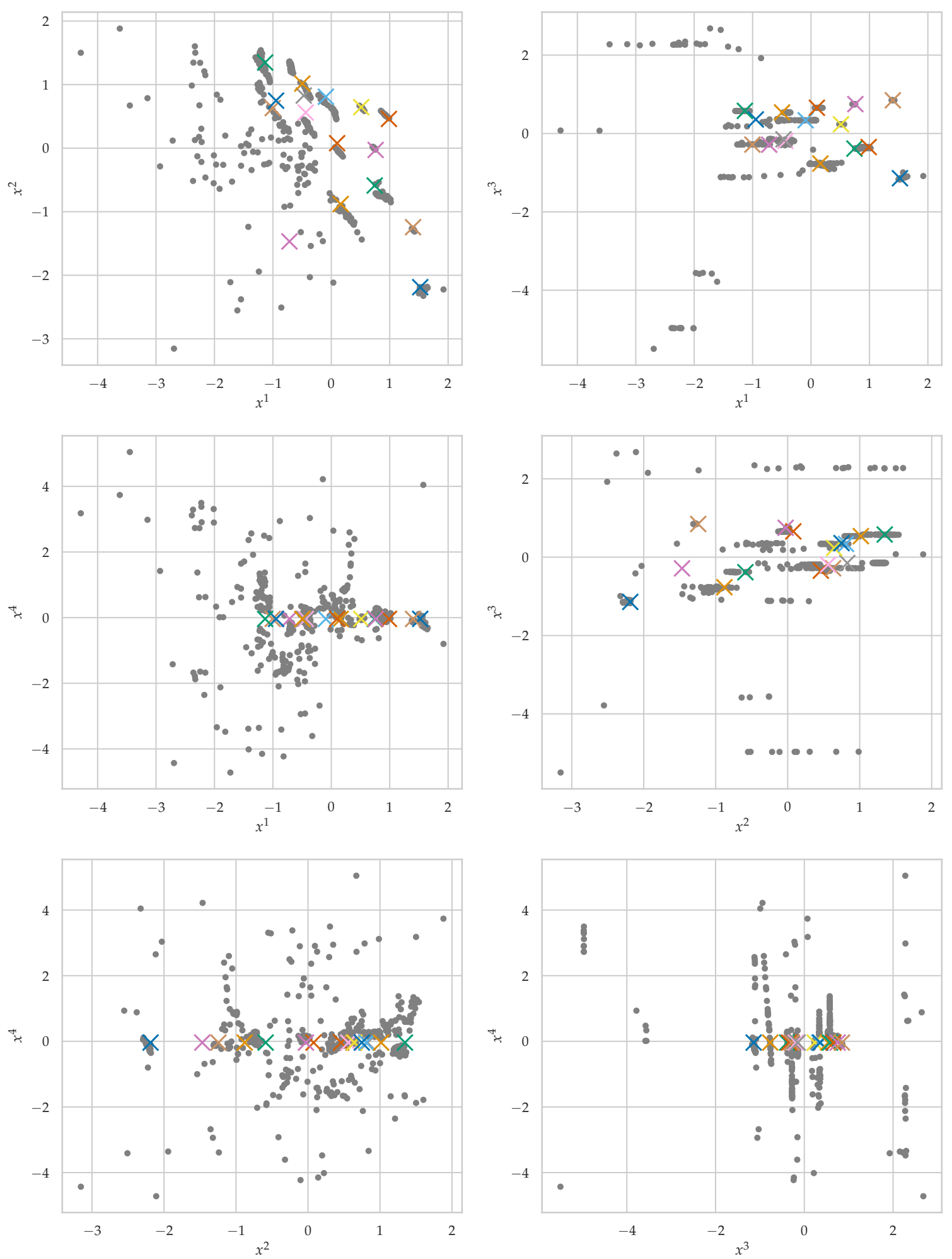

Figura 3.20 - Diagramas de dispersão para cada par de componentes do vetor $\mathbf{x}=\left(x^{1}, x^{2}, x^{3}, x^{4}\right) \in$ $\mathcal{X}$ após padronização dados (Equações 3.40-3.43 e 3.46). O pontos pretos são componentes amostradas pelo KBDM para um sinal simulado com ruído $(\sigma=0.03)$ a partir dos parâmetros da Tabela 2.1. Os marcadores $\mathbf{X}$ coloridos representam os valores verdadeiros dos parâmetros.

Fonte: Elaborada pelo autor. 


\subsection{Ajuste iterativo}

Após a etapa de agrupamento, a média do respectivo cluster é utilizada como estimativa final para cada linha. A qualidade desta estimativa pode ser aferida através de medidas de dispersão ou índices mais específicos na avaliação de clusters, como é o caso da medida silhueta. (75) Durante o procedimento, há a possibilidade de que alguma linha verdadeira não seja detectada pelo algoritmo de agrupamento. Isto pode ocorrer em função da ausência de detecções para a linha pelo KBDM, normalmente causada por alta sobreposição e baixa relação sinal-ruído da linha. Tanto o descarte de linhas de baixa qualidade, quanto a ausência de detecção de um determinado pico resultará em sinais residuais de alta amplitude. O sinal residual desejado deve conter apenas ruído e qualquer artefato presente pode indicar problemas nas estimativas obtidas. (13)

Um novo processo de amostragem utilizando o KBDM e o agrupamento das linhas resultantes podem ser realizados no sinal residual para obtenção das componentes residuais. Nesta etapa, dado que o número de componentes genuínos é menor, o grau de sobreposição dos picos também é reduzido. Picos com amplitude menores se tornam mais significativos. Em contrapartida, a imprecisão das estimativas a partir da execução anterior pode causar a presença de artefatos no sinal residual.

Esta etapa pode ser repetida até uma quantidade fixa de iterações ou quando o resíduo atingir o patamar desejado. Quando o algoritmo de agrupamento não for capaz de obter mais clusters, o processo deve ser interrompido.

Seja $\Gamma\left(\Pi^{(j)}\right)$ o conjunto de linhas promediadas a partir do particionamento $\Pi^{(j)}$ durante a j-ésima iteração, o conjunto total de linhas estimadas até a $i$-ésima iteração é dado por

$$
\Gamma^{(i)}=\bigcup_{j=1}^{i} \Gamma\left(\Pi^{(j)}\right)
$$

O sinal residual $\left(\tilde{C}^{r e s}\right)^{(i)}$ é definido, portanto, pela diferença entre o sinal de entrada $\tilde{C}_{n}$ sinal total estimado $\tilde{C}_{n}^{\prime}\left(\Gamma^{(i)}\right)$, tal que

$$
\left(\tilde{C}_{n}^{r e s}\right)^{(i)}=\tilde{C}_{n}-\tilde{C}_{n}^{\prime}\left(\Gamma^{(i)}\right)
$$

$\left(\tilde{C}^{r e s}\right)^{(i)}$ é utilizado durante a iteração sucessiva na construção do particionamento $\Pi^{(i+1)}$, ou seja, $\left(\tilde{C}_{n}^{r e s}\right)^{(i)}$ se torna o sinal de entrada no escopo desta iteração; e o processo se repete 
enquanto

$$
\frac{1}{N} \sum_{n=0}^{N-1}\left\|\left(\tilde{C}_{n}^{r e s}\right)^{(i)}\right\|>\epsilon_{t o l}
$$

onde $\epsilon_{t o l}$ é a tolerância residual desejada; ou enquanto $i<i_{\max }$, sendo $i_{\max }$ o número máximo de iterações permitidas.

\section{$3.6 \quad L L C-K B D M$}

Nesta seção, o conteúdo apresentado neste capítulo será sintetizado em uma versão final para o algoritmo proposto. Este algoritmo recebe o nome LLC-KBDM, do inglês Line List Clustering KBDM (Método da Diagonalização na Base de Krylov com Agrupamento de Linhas).

O fluxograma ilustrado pela Figura 3.21 descreve a interação entre os mecanismos discutidos anteriormente. É importante notar a modularidade do algoritmo, onde seus respectivos componentes podem ser projetados ou otimizados de acordo com os requisitos de cada aplicação. O processo de amostragem pode ser realizado utilizando a abordagem de adição de pseudo-ruído ou múltiplos truncamentos do sinal. O agrupamento de linhas pode ser realizado por diversos algoritmos existentes na literatura e as medidas de similaridade entre as linhas podem ser alteradas. Por sua vez, a promediação pode ser realizada utilizando médias, medianas ou alguma outra medida de sumarização. Inúmeros critérios de verificação de qualidade, bem como combinações entre eles podem ser adotados. Por fim, a mesma diversidade existe na escolha de critérios de parada ou verificação de convergência, que podem ser medidas globais sobre o sinal, como os que foram discutidos nas seções anteriores; ou ainda poderão levar em consideração a otimização apenas em uma dada região espectral.

Com base na experiência adquirida durante o desenvolvimento do projeto, algumas escoIhas foram sugeridas. Seria impossível explorar todas as alternativas dentro do escopo deste trabalho, mas a recomendação de que é possível explorá-las na solução de novos problemas deve ser evidenciada.

O pseudo-código para o LLC-KBDM é apresentado pelo Algoritmo 1. 


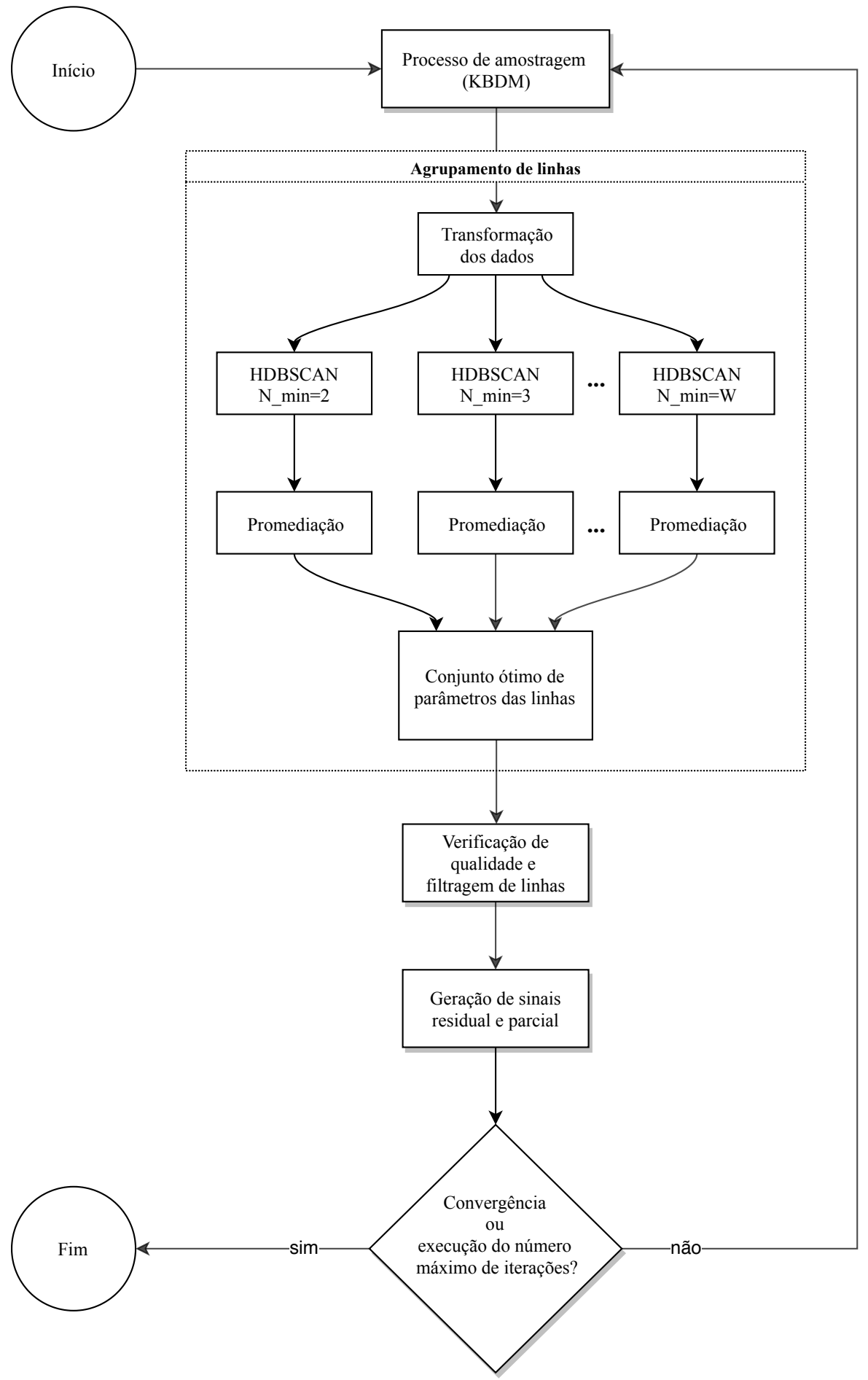

Figura 3.21 - Fluxograma do algoritmo LLC-KBDM. O processo de amostragem utilizando o KBDM é seguido pela otimização do agrupamento de linhas. Após a escolha do melhor conjunto de parâmetros, as linhas filtradas são utilizadas na construção do sinal parcial. A partir da diferença entre o sinal parcial total estimado e o sinal de entrada, o teste de convergência é aplicado. Se a convergência for alcançada, o algoritmo é encerrado. Caso contrário, o sinal residual é processado novamente e as etapas anteriores se repetem até que o número máximo de iterações seja excedido ou a convergência seja obtida.

Fonte: Elaborada pelo autor. 


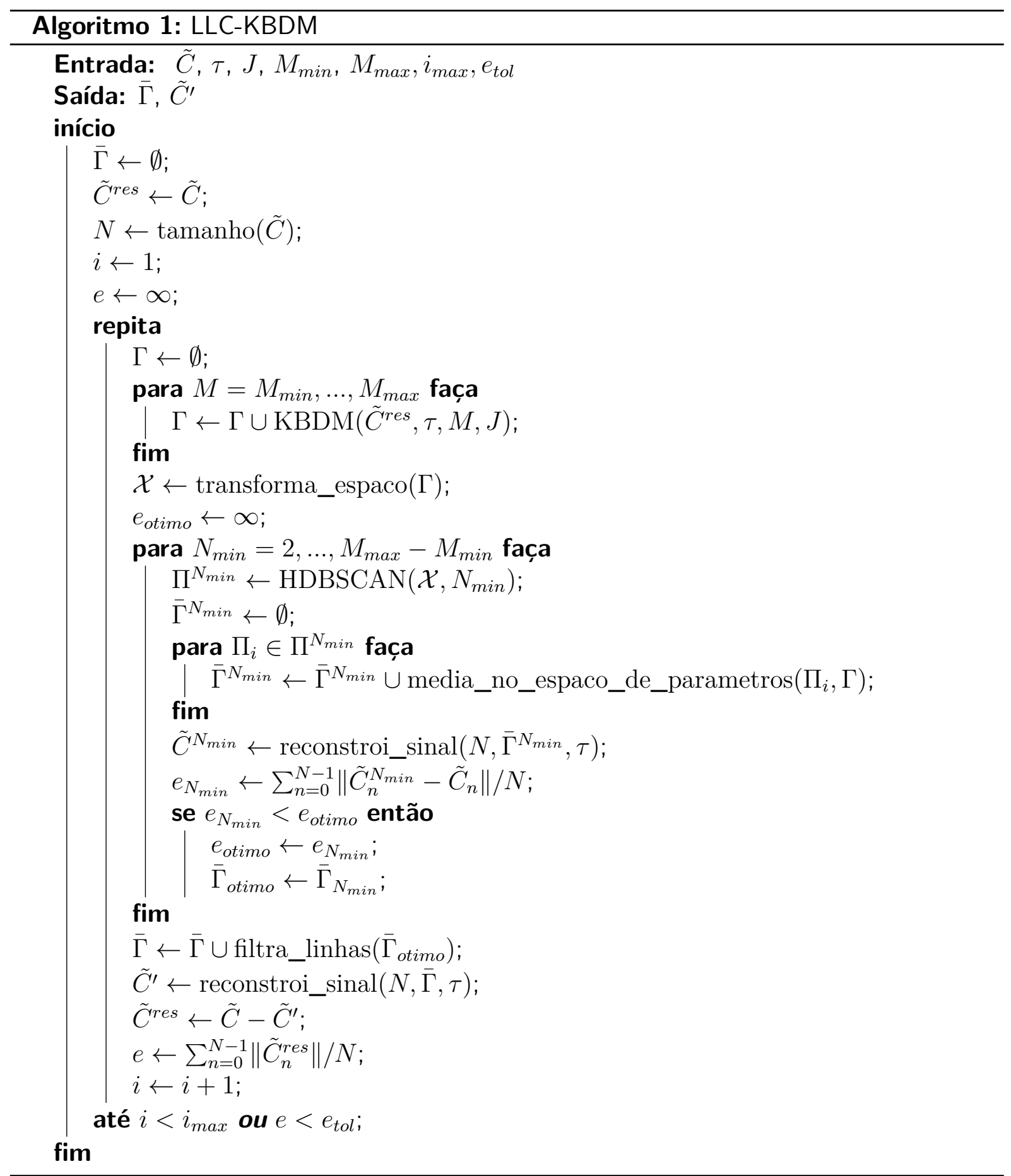




\section{Desenvolvimento de ferramentas computacionais para o KBDM e LLC-KBDM}

O KBDM é um método pouco utilizado na literatura e o LLC-KBDM é proposto originalmente neste trabalho. Para que o uso destas técnicas seja incentivado é necessário oferecer ferramentas computacionais onde o usuário interessado possa utilizá-las sem necessariamente possuir todo o conhecimento específico para desenvolver a própria implementação. A capacidade desses programas computacionais serem utilizados através do principais sistemas operacionais é um atrativo adicional desejado. Os códigos desenvolvidos durante a realização deste trabalho estarão disponíveis em https://github.com/danilomendesdias/llckbdm ou através de solicitação ao autor.

Outro aspecto importante é permitir o uso dessas ferramentas por diferentes tipos de usuários. Por exemplo, usuários que não possuem conhecimento de programação ou aqueles que estejam interessados apenas em operar os métodos na execução de uma determinada tarefa podem achar mais conveniente o uso das ferramentas através de uma interface gráfica. Por outro lado, pesquisadores ou desenvolvedores de software científico podem estar interessados em modificar ou integrar apenas módulos que efetuam os cálculos numéricos envolvidos em suas próprias ferramentas.

Dentro destes dois cenários, as ferramentas foram dividas em duas camadas:

1. Biblioteca computacional que contém apenas as tarefas envolvendo computação numérica associadas ao método. Esta camada é denominada API (Application User Interface);

2. Interface gráfica com os requisitos mínimos para o uso dos métodos através do consumo interno da API. Este programa multiplataforma recebeu o nome MRSuppresor.

A API é composta pelos módulos kbdm, llc_kbdm, sig_gen e metrics. Os dois primeiros módulos fornecem as rotinas principais para os métodos KBDM e LLC-KBDM, respectivamente; as funcionalidades para geração de sinais simulados e outros mecanismos relacionados 
a reconstrução do sinal através do parâmetros das linhas estão contidas em sig_gen; e as medidas de erro e qualidade dos clusters estão implementadas no módulo metrics.

A linguagem principal adotada foi o Python 3.7, na última versão estável disponível no momento da escrita deste trabalho. Embora não seja uma restrição, é recomendado que a distribuição do interpretador do Python, bem como os pacotes utilizados pela API sejam adquiridos através do ambiente conda configurado para consumir apenas pacotes disponibilizados pelo canal conda-forge. (86) Esta abordagem evita a necessidade de compilação local das bibliotecas numéricas utilizadas e reduz problemas de incompatibilidade entre os pacotes.

Embora o Python seja uma linguagem interpretada, as principais bibliotecas numéricas utilizadas neste projeto, como é o caso NumPy, SciPy, Pandas, scikit-learn e hdbscan requerem compilação. (64, 83, 87-90) Isso ocorre porque o Python permite a utilização de rotinas compiladas em outras linguagens mais performáticas computacionalmente, como é o caso do Fortran, C ou C++. Deste modo, em operações onde a sobrecarga de chamada desses módulos compilados é relativamente menor do que o tempo interno de execução da rotina compilada, é possível obter desempenhos comparáveis às próprias linguagens compiladas.

Duas operações operações fundamentais do algoritmo do KBDM (ver Seção 2.4.2) são a decomposição SVD da matriz de sobreposição (Equação 2.105) e a diagonalização da matriz reduzida $\tilde{G}$ (Equação 2.135). As implementações utilizadas são, respectivamente, numpy. linalg. svd e numpy. linalg. eig da biblioteca NumPy. De fato, essas funções apenas encapsulam as chamadas _gesdd e _.geev especificadas pela Linear Algebra PACKage (LAPACK). (91) O pacote NumPy disponibilizado por padrão pelo canal conda-forge é compilado com a implementação proprietária da LAPACK contida na Math Kernel Library (MKL), otimizada para os processadores da Intel. (92)

No desenvolvimento do software MRSuppressor, as principais ferramentas utilizadas foram: PyQt (93), no encapsulamento do arcabouço de interface gráfica, Qt, para o Python; PyQtGraph (94), para visualização dos sinais; Suspect (95), na leitura dos dados de espectroscopia em formatos proprietários (Philips, Siemens, Bruker); Pandas, na manipulação de arquivos e estruturas de dados tabulares.

A interface gráfica quanto e a API foram desenvolvidas utilizando o processo Test Driven Development (TDD) com auxílio do arcabouço de testes pytest. (96) Dezenas de testes unitários foram implementados e as versões foram continuamente integradas e testadas nos sistema Linux, Windows e macOS.

As Figuras 4.1 e 4.2 mostram exemplos de casos de uso do software MRSuppressor. 


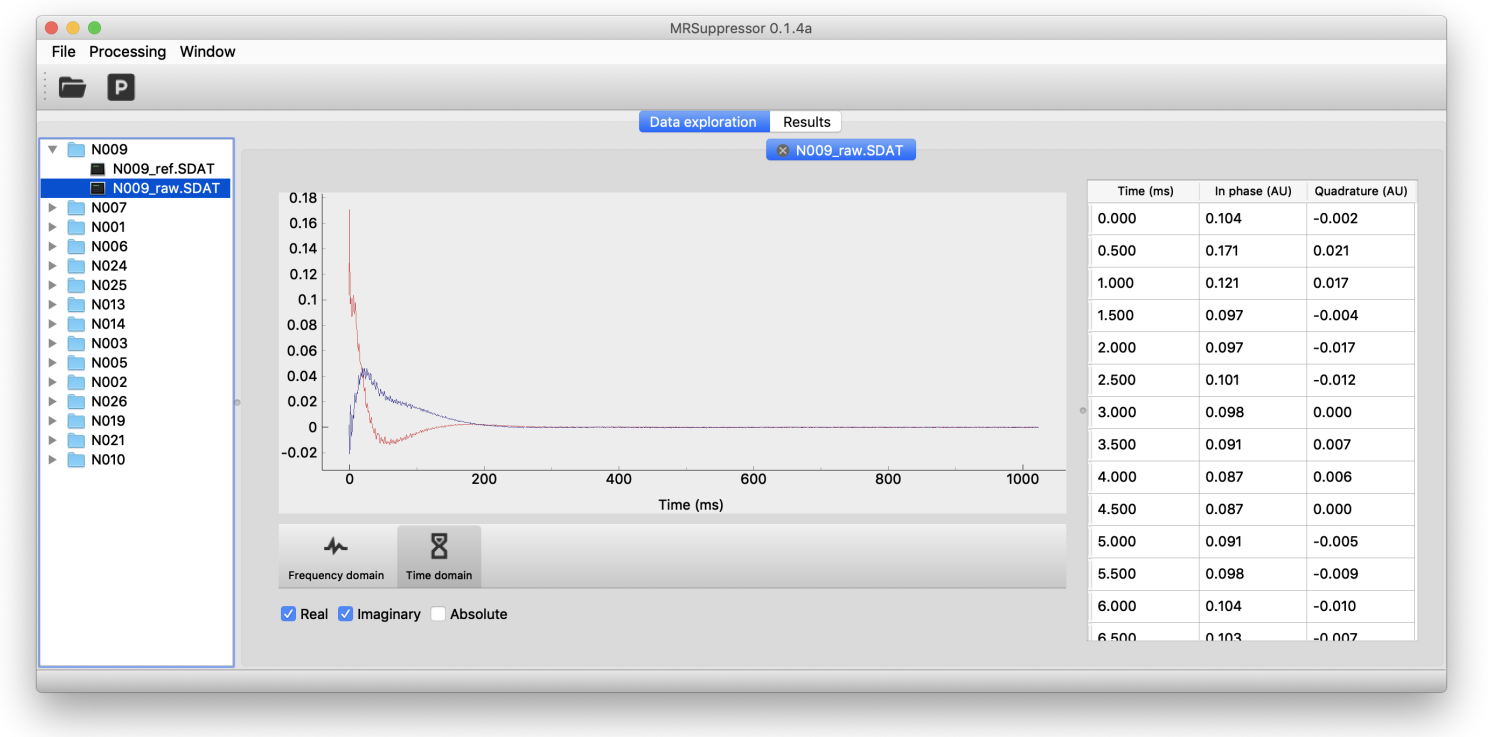

Figura 4.1 - Demonstração do MRSuppressor na leitura de dados de ERM in vivo em formato comercial Philips (SDAT/SPAR). Após a escolha do diretório raiz, o usuário pode visualizar o dado no domínio do tempo ou da frequência. As componentes reais, imaginárias e também a magnitude do dado representado em notação complexa podem ser simultaneamente visualizadas.

Fonte: Elaborada pelo autor.

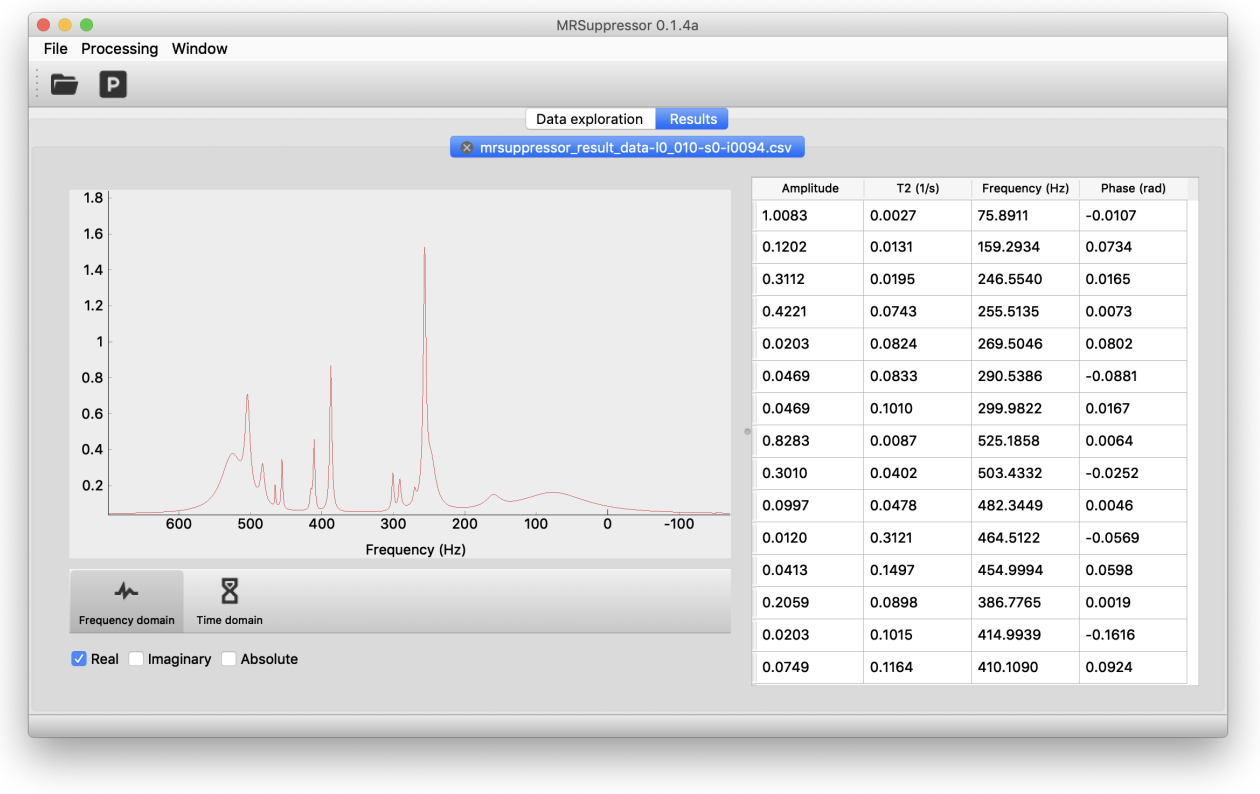

Figura 4.2 - Visualização das estimativas do espectro e parâmetros das linhas processadas pelo KBDM no MRSuppressor para sinal ruidoso simulado através dos parâmetros da Tabela 2.1 .

Fonte: Elaborada pelo autor. 


\section{Aplicações e Resultados}

\subsection{Sinal simulado com adição de ruído}

Os parâmetros que descrevem 16 picos de um sinal ideal de espectroscopia por RM na região do cérebro humano apresentados pela Tabela 2.1 foram utilizados na construção de 100 sinais ruidosos para cada um dos níveis $\sigma=0.01,0.015,0.024,0.025,0.03,0.035,0.04,0.045$, 0.05 , totalizando 900 espectros. O número de pontos complexos de cada sinal é $N=2048$ e o intervalo de amostragem é $\tau=5 \mathrm{~ms}$.

Foram avaliados os seguintes métodos: o KBDM (Capítulo 2), o LLC-KBDM, proposto originalmente neste trabalho (Capítulo 3); e o $\operatorname{HLSVD-PRO}(19,29)$. O HLSVD-PRO representa o estado da arte na literatura para a reconstrução de componentes que podem ser ajustados através da Equação 2.1 em sinais de espectroscopia por RM.

A implementação disponível no software jMRUI (97-98) para HLSVD-PRO foi utilizada. Para o KBDM e o LLC-KBDM, os sinais foram processados através das implementações em Python desenvolvidas durante a execução deste trabalho e que foram discutidas no Capítulo 4.

O HLSVD-PRO e o KBDM fazem uso de suas respectivas formulações de matrizes com simetria de Hankel, construídas a partir do sinal no domínio do tempo. No HLSVD-PRO, a matriz de Hankel utilizada possui $M$ linhas e $L$ colunas; enquanto o KBDM utiliza duas matrizes quadradas $M \times M, \tilde{U}^{0}$ e $\tilde{U}^{1}$, seguido da decomposição em valores singulares da matriz de sobreposição utilizando-se apenas as dimensões associadas aos $J$ valores singulares mais significativos (ver Seção 2.4.2). Esta matriz foi denotada pelo símbolo $\tilde{U}^{0}$ e denominada por matriz de sobreposição no contexto do KBDM (ver Seção 2.3).

Para que a comparação entre os métodos fosse justa, ambos foram processados a partir dos $N=600$ primeiros pontos complexos. O número de linhas das matrizes de Hankel também foram os mesmos $M=300$, resultando em duas matrizes quadradas $300 \times 300$ para o KBDM e uma matriz $300 \times 301$ para o HLSVD-PRO. A pequena diferença entre as dimensionalidades ocorre em função das distintas restrições envolvendo o número de pontos processado em cada método. 
Dois valores distintos, $J=16$ e $J=25$, foram utilizados para avaliar o impacto da redução de dimensionalidade após o truncamento das matrizes obtidas pela decomposição em valores singulares. O primeiro valor considera exatamente o número de componentes contidos no sinal, enquanto o segundo caracteriza o sobreajuste do sinal.

Por sua vez, o LLC-KBDM foi amostrado em torno do mesmo valor utilizado pelos outros dois métodos por meio de múltiplos truncamentos descritos pelos parâmetros $M=275, \ldots, 324$ ( $W=50$ amostras). Os mesmos valores $J=16$ e $J=25$ foram utilizados no truncamento após a decomposição SVD da matriz $\tilde{U}^{0}$.

O número máximo de iterações sobre os resíduos parciais (ver Seção 3.5) foi de $i_{\max }=3$. O LLC-KBDM foi implementado de maneira a interromper a execução quando o algoritmo de agrupamento não for capaz de obter clusters que sejam aceitos pelos critérios de filtragem ou quando não for possível obter estimativas que reduzam o erro global. A condição de parada por tolerância fixa do erro não foi utilizada.

A região espectral de interesse foi definida de 0 a $600 \mathrm{~Hz}(0$ a $4.7 \mathrm{ppm})$. Todas as linhas fora dessa região espectral, com amplitude inferior a $10^{-3}$ ou com taxas de relaxação negativas foram filtradas dos resultados de todos os métodos.

\subsubsection{Resultados e discussões}

A Figura 5.1 mostra o desvio padrão do resíduo do sinal estimado em relação ao sinal simulado (sem adição de ruído) no domínio do tempo para cada um dos métodos em função dos níveis de ruído determinados por $\sigma=0.01,0.015,0.02,0.025,0.03,0.035,0.04,0.045$, 0.05 .

Para os três primeiros níveis de ruído $(\sigma=0.01,0.015,0.02)$, pouca diferença de desempenho para a métrica adotada é observada. Isso ocorre em função dos ajustes de alta qualidade em todos os casos.

A partir do nível $\sigma=0.025$, o LLC-KBDM com três iterações utilizando $J=16$ (método A) é superior aos métodos KBDM e HLSVD-PRO com este mesmo truncamento após o SVD (métodos $\mathbf{B}$ e $\mathbf{C}$ ), sendo comparável aos métodos $\mathbf{D}, \mathbf{E}$ e $\mathbf{F}$, que utilizam $J=25$. Neste cenário, a intensidade do ruído no sinal adiciona valores singulares espúrios significativos que se sobrepõem aos genuínos. Por não possuírem coerência no espaço transformado de dados durante o agrupamento das linhas, estes picos espúrios são eliminados e o processo iterativo 


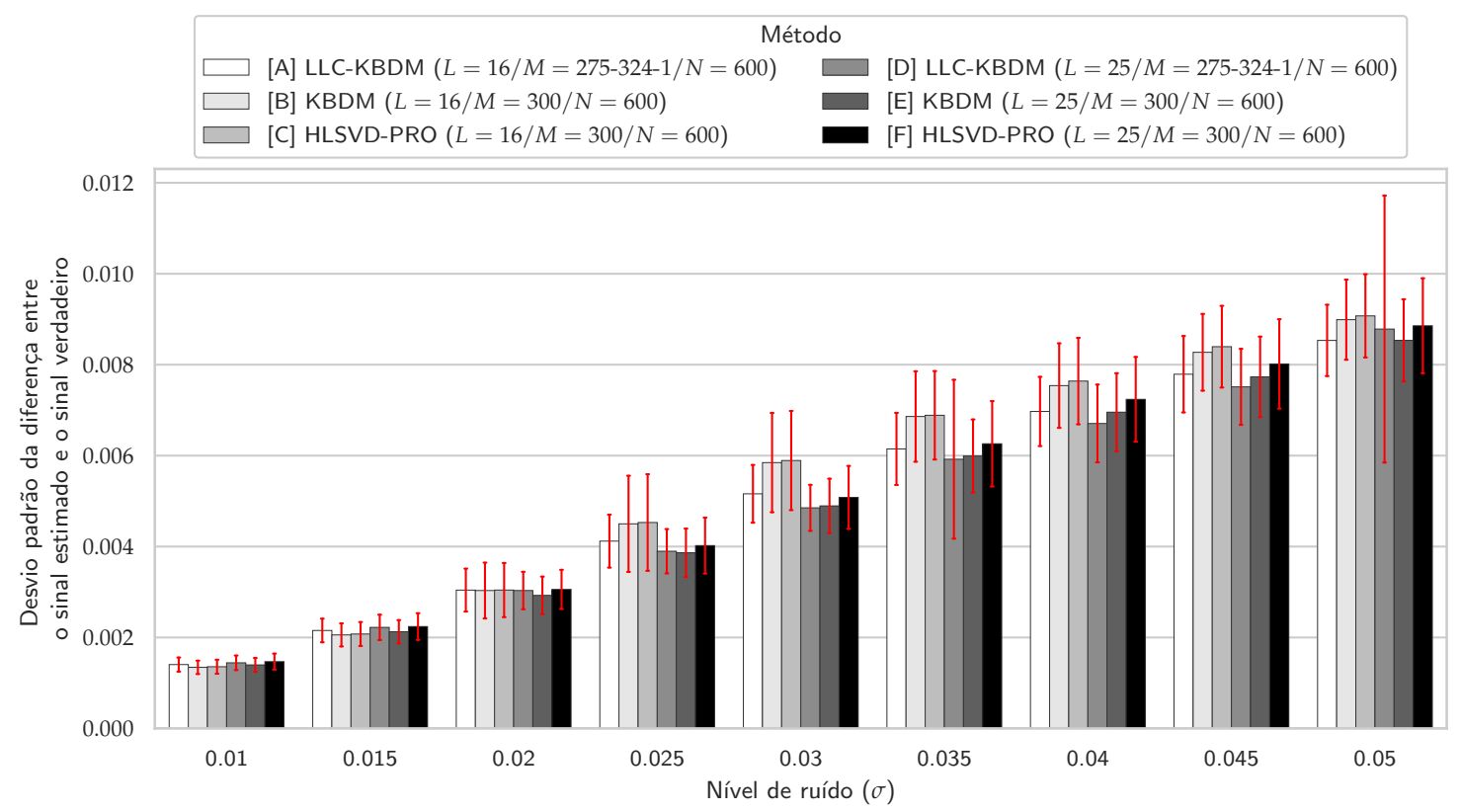

Figura 5.1 - Desvio padrão do sinal residual calculado a partir da diferença entre o sinal estimado e o sinal verdadeiro (gerado a partir da Tabela 2.1, sem adição de ruído) em função dos níveis de ruídos sintéticos caracterizados pelos desvios padrões $\sigma=0.01,0.015$, $0.024,0.025,0.03,0.035,0.04,0.045,0.05$.

Fonte: Elaborada pelo autor.

fornece uma nova chance para que as componentes genuínas remanescentes no resíduo possam ser observadas.

A configuração $J=25$ (D, E e F) representa a priori soluções sobredeterminadas. Portanto, esta configuração permite que componentes espúrias e genuínas sejam estimadas simultaneamente. Pequenas reduções de desempenho em relação aos métodos ( $\mathbf{A}, \mathbf{B}$ e $\mathbf{C})$ em função dessa sobredeterminação são observadas nos sinais com melhores relações sinal-ruído $(\sigma=0.01$ e 0.015$)$. Para os demais níveis, uma tendência a melhores ajustes para $J=25$ é observada em relação a configuração de ordem $J=16$.

Uma exceção para este último comportamento se manifesta através do desempenho do LLC-KBDM para o nível $\sigma=0.05$ (D), indicando provável instabilidade do método quando há muito ruído. A alta variância dos parâmetros das linhas estimadas dificulta o processo de agrupamento e os erros acumulados entre as iterações do algoritmo também prejudicam o ajuste final. A tendência de um desempenho superior do agrupamento na presença de um número reduzido de linhas é evidenciado pelo fato de que, neste caso, o LLC-KBDM com $J=16$ (método $\mathbf{A}$ ) se mostrou superior ao método $\mathbf{D}$.

De maneira geral, o LLC-KBDM possui uma tendência a realizar melhores ajustes em regiões intermediárias de ruído do que KBDM e ao HLSVD-PRO. No entanto, alguns casos 
de instabilidade no algoritmo são observados quando os parâmetros caracterizam condições de sobreajuste $(\sigma=0.035$ e $\sigma 0.05 \mathrm{com} J=25)$. Uma pequena superioridade dos resultados obtidos pela métrica adotada para o KBDM em relação ao HLSVD-PRO é sistematicamente observada em todas as configurações, para todos os níveis de ruído.

Para estes mesmos resultados, a Figura 5.2 apresenta uma visão baseada na acurácia do número de picos corretamente estimados por cada um dos métodos em função do níveis de ruído. Esta análise tem a característica de não considerar o impacto da presença dos picos espúrios estimados por cada um dos métodos.

Embora as diferenças não sejam muito significativas entre os métodos com o mesmo valor do parâmetro $J$, os métodos $\mathbf{A}$ e $\mathbf{D}$ apresentam ligeira vantagem em relação aos métodos sem o uso de agrupamento de linhas com, respectivamente, $J=16$ e $J=25$, sendo o LLC-KBDM com $J=25$ (D) o mais acurado em praticamente todos os níveis de ruído.

As Figuras 5.3-5.11 apresentam as taxas de sucesso nas estimativas de cada pico contido no sinal para cada método. Cada figura apresenta os resultados dos respectivos níveis de ruído.

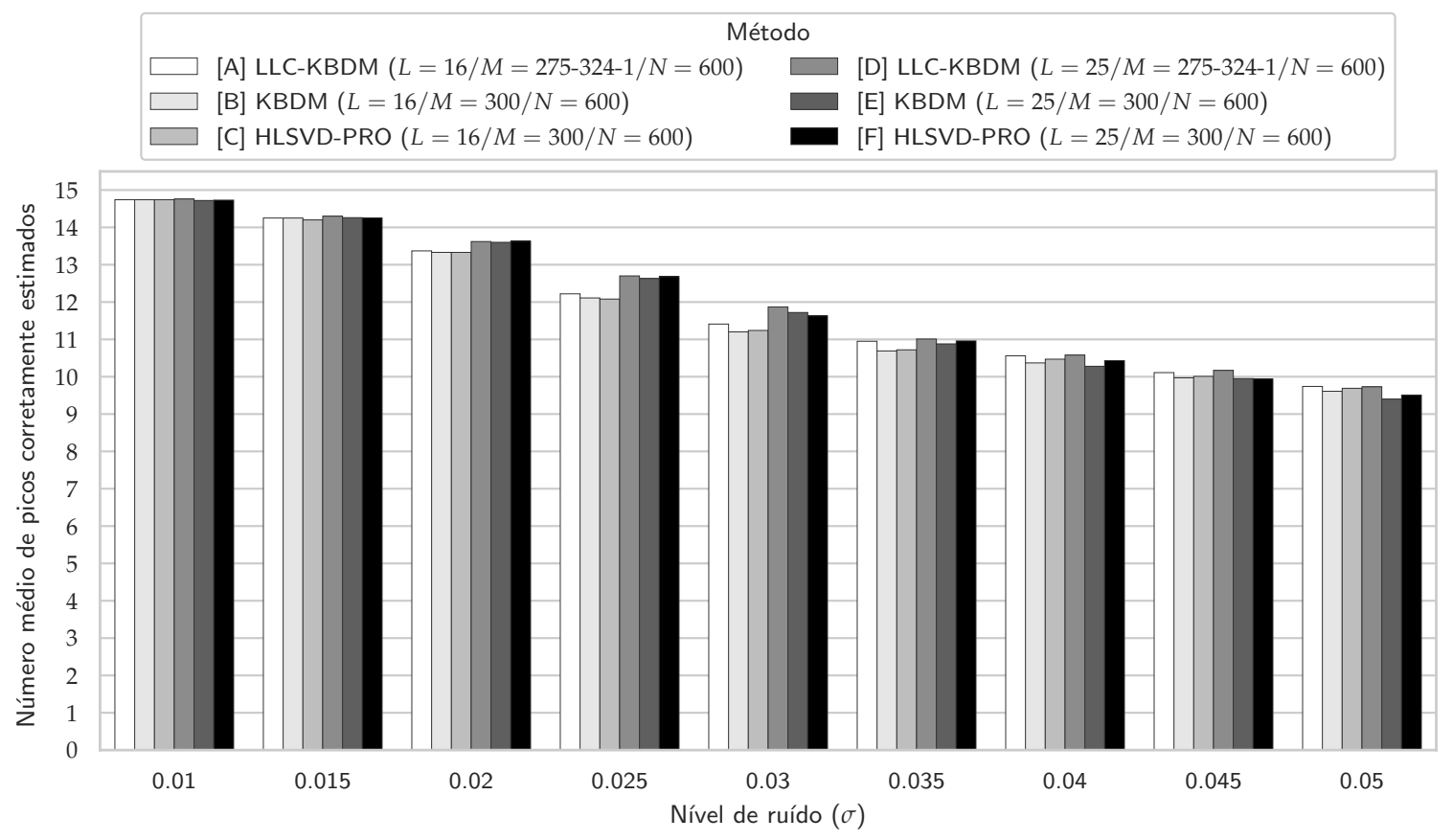

Figura 5.2 - Número médio de linhas corretamente estimadas em função dos níveis de ruídos para cada método estudado.

Fonte: Elaborada pelo autor. 

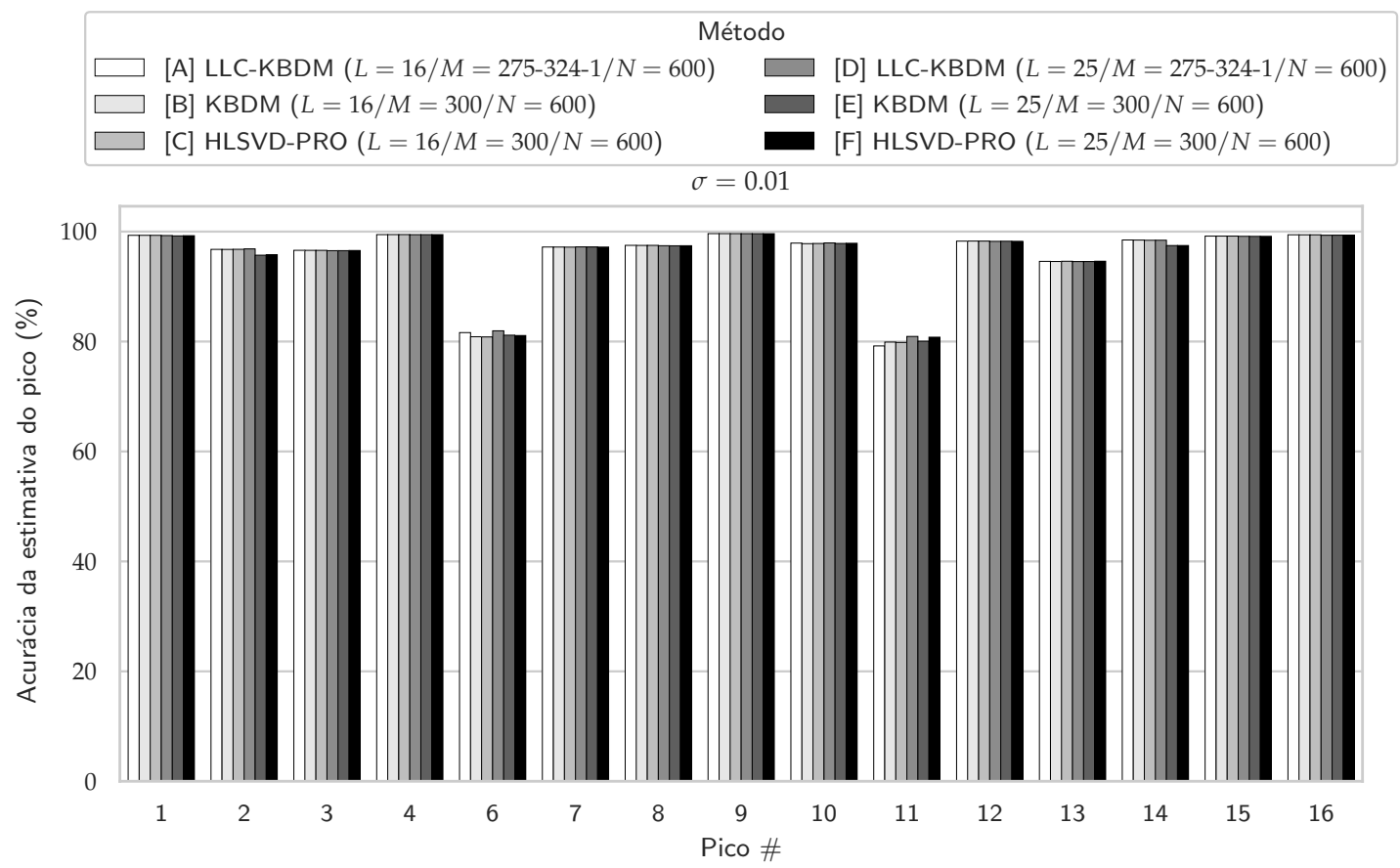

Figura 5.3 - Acurácia das estimativas bem sucedidas de cada pico para o nível de ruído caracterizado por $\sigma=0.01$ em função do método utilizado. Por não ter sido detectado em nenhum dos casos, o pico \#5 foi omitido do gráfico.

Fonte: Elaborada pelo autor.

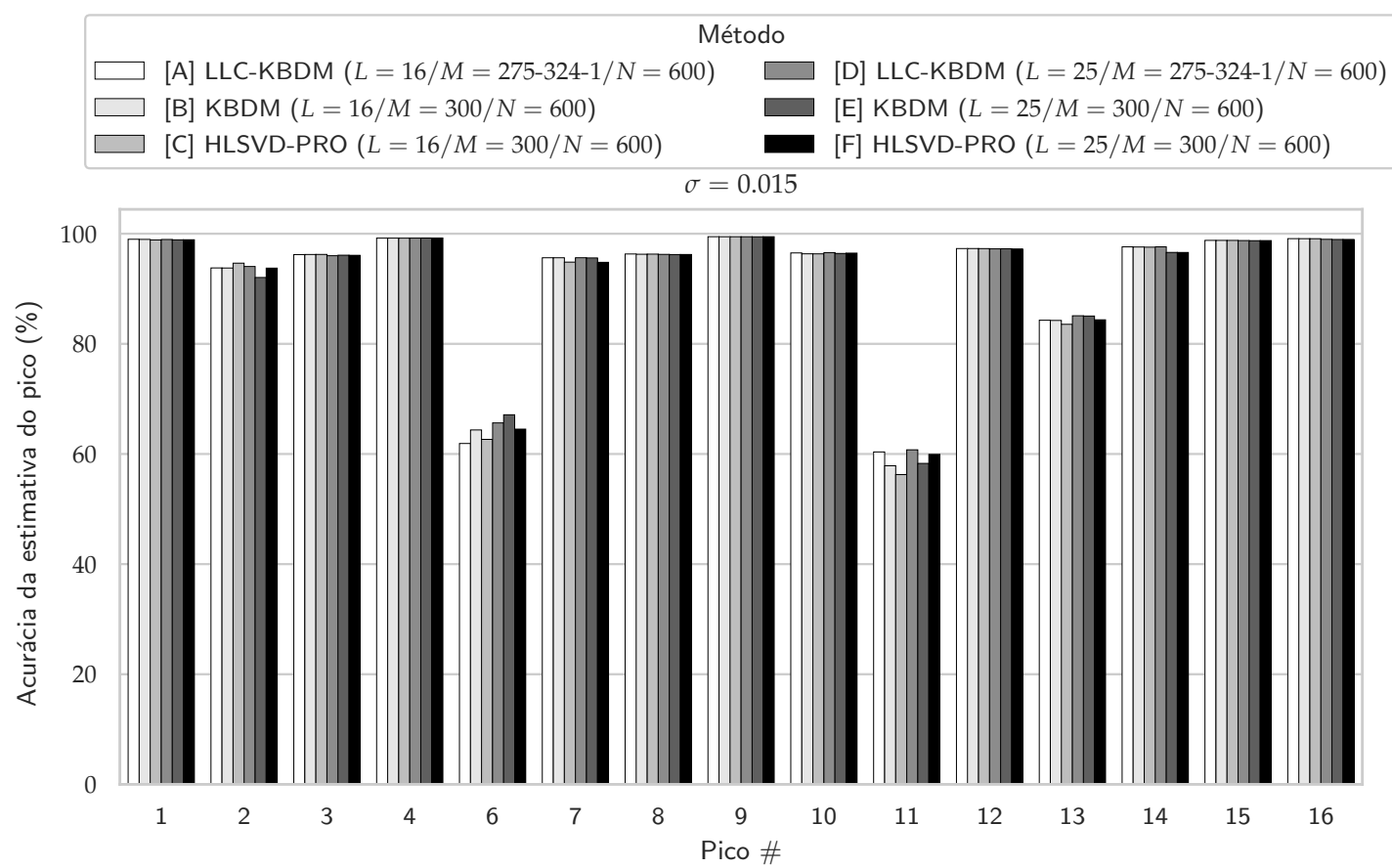

Figura 5.4 - Acurácia das estimativas bem sucedidas de cada pico para o nível de ruído caracterizado por $\sigma=0.015$ em função do método utilizado. Por não ter sido detectado em nenhum dos casos, o pico \#5 foi omitido do gráfico.

Fonte: Elaborada pelo autor. 


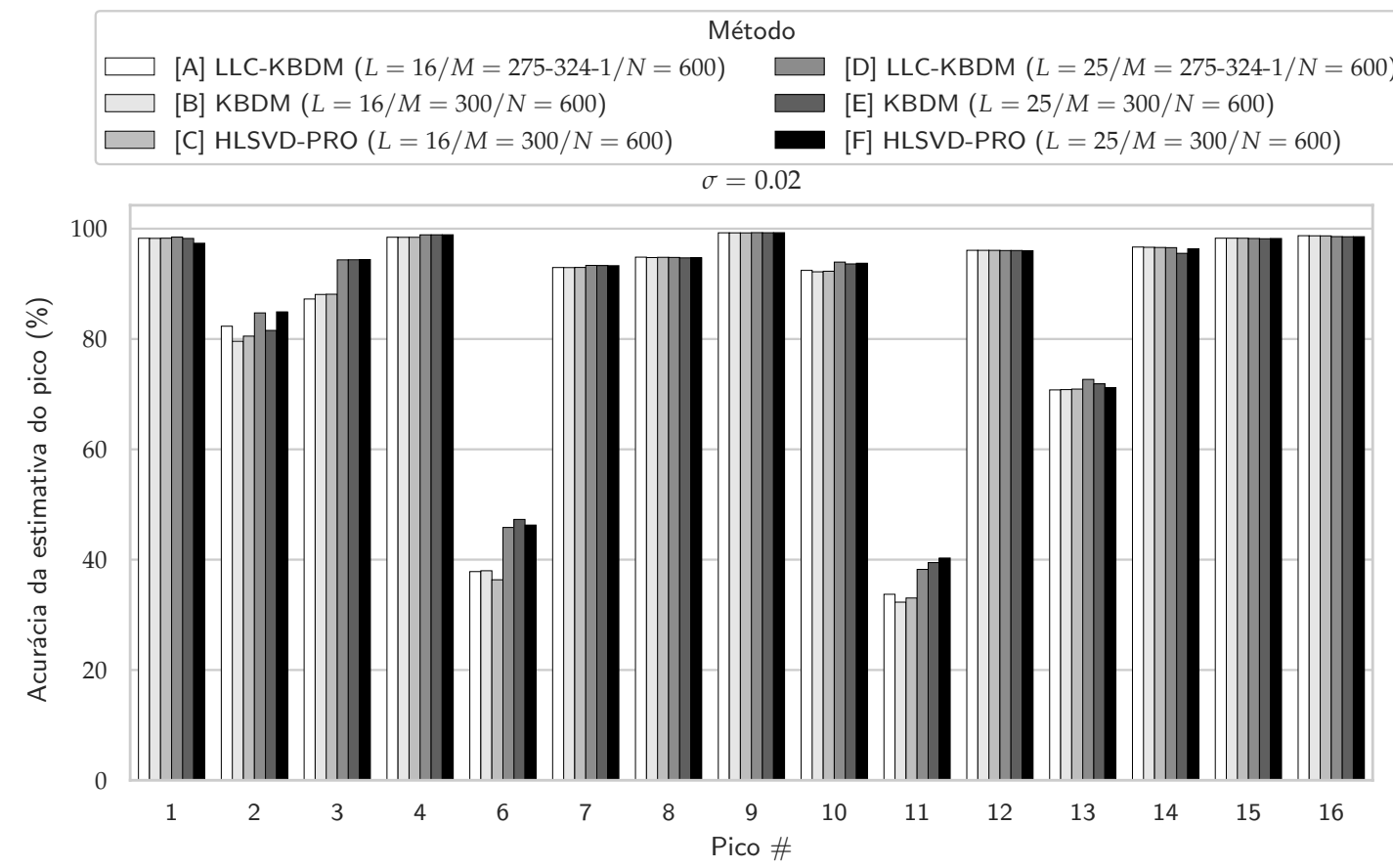

Figura 5.5 - Acurácia das estimativas bem sucedidas de cada pico para o nível de ruído caracterizado por $\sigma=0.02$ em função do método utilizado. Por não ter sido detectado em nenhum dos casos, o pico \#5 foi omitido do gráfico.

Fonte: Elaborada pelo autor.

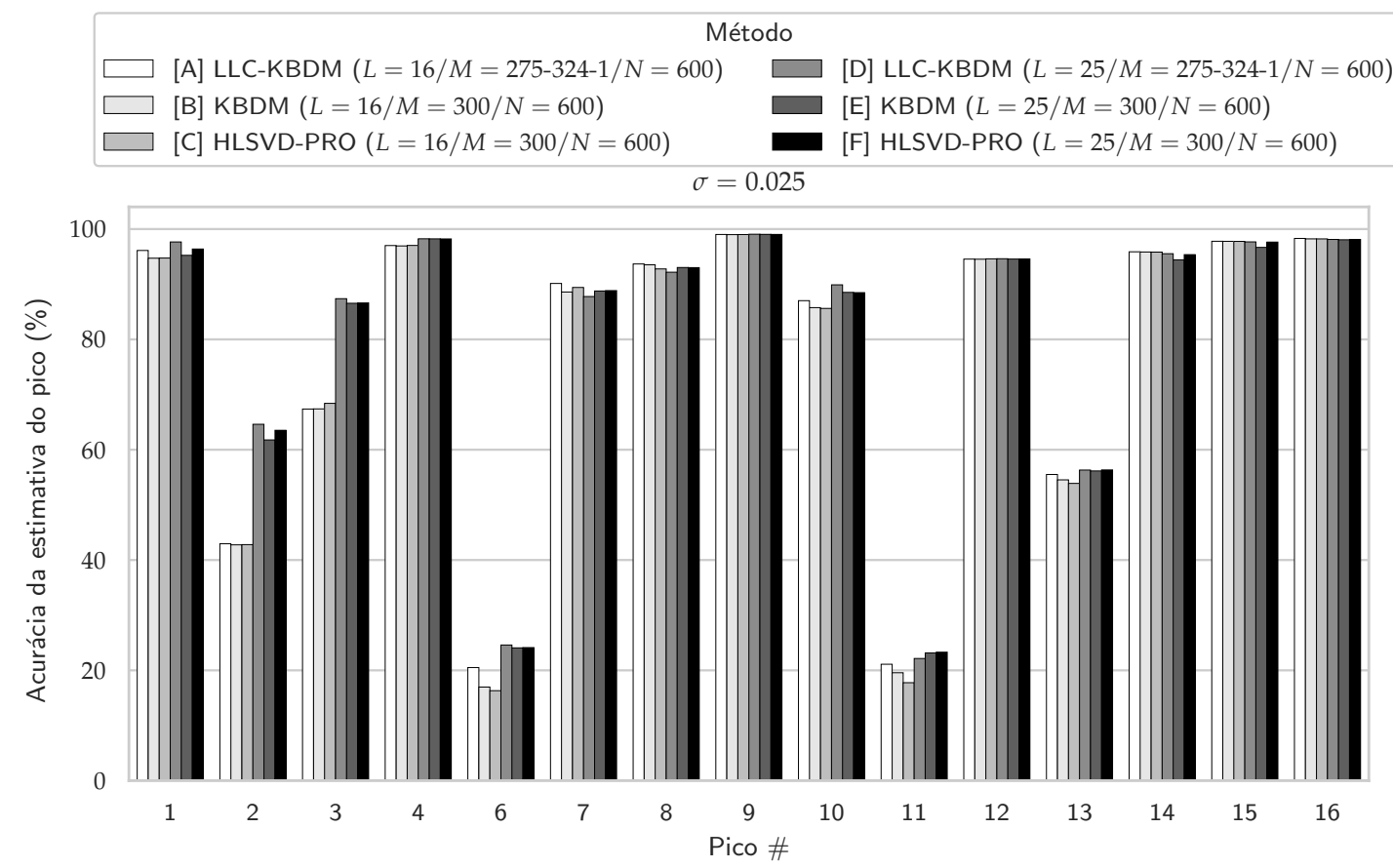

Figura 5.6 - Acurácia das estimativas bem sucedidas de cada pico para o nível de ruído caracterizado por $\sigma=0.025$ em função do método utilizado. Por não ter sido detectado em nenhum dos casos, o pico \#5 foi omitido do gráfico.

Fonte: Elaborada pelo autor. 




Figura 5.7 - Acurácia das estimativas bem sucedidas de cada pico para o nível de ruído caracterizado por $\sigma=0.03$ em função do método utilizado. Por não ter sido detectado em nenhum dos casos, o pico \#5 foi omitido do gráfico.

Fonte: Elaborada pelo autor.

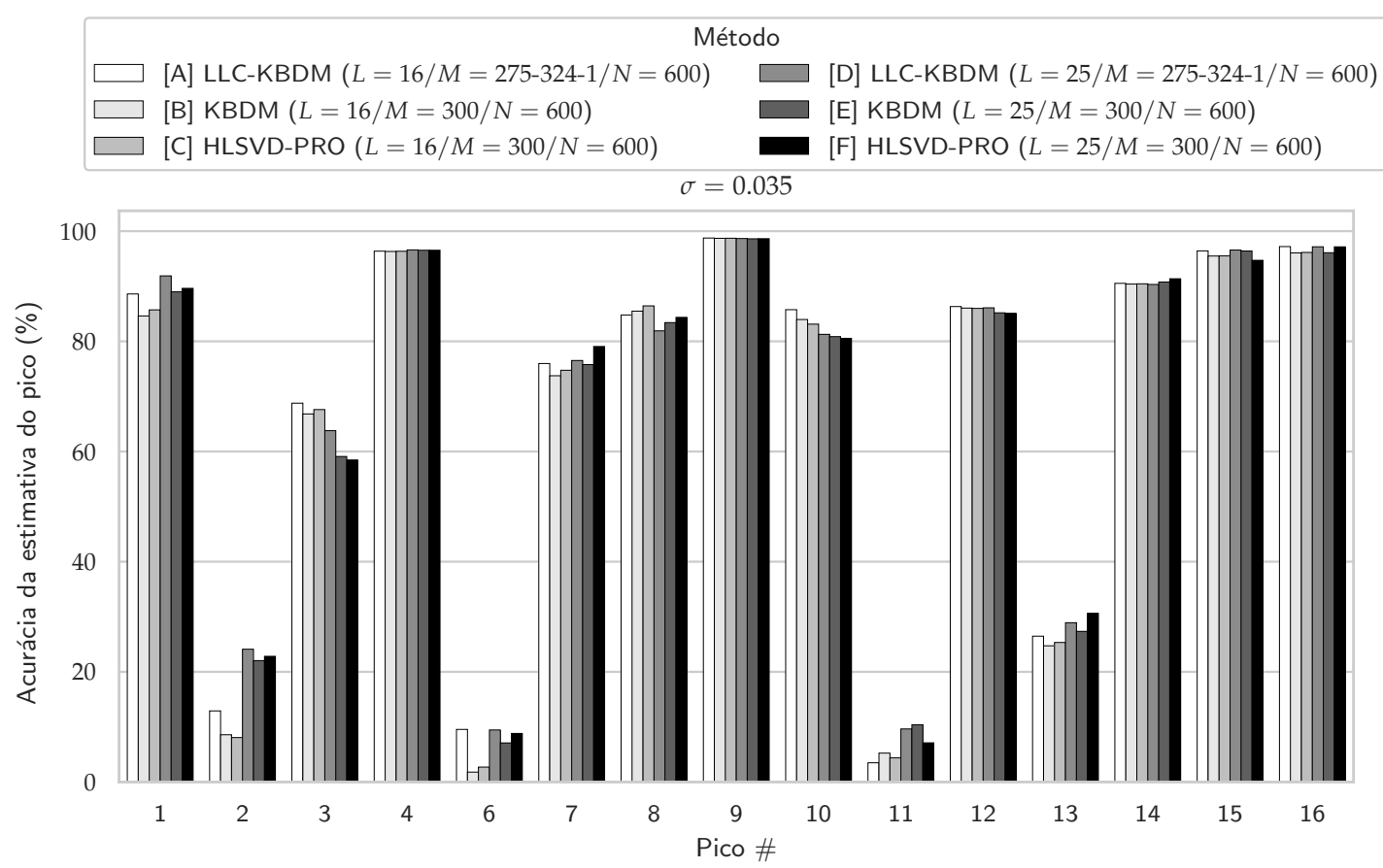

Figura 5.8 - Acurácia das estimativas bem sucedidas de cada pico para o nível de ruído caracterizado por $\sigma=0.035$ em função do método utilizado. Por não ter sido detectado em nenhum dos casos, o pico \#5 foi omitido do gráfico.

Fonte: Elaborada pelo autor. 


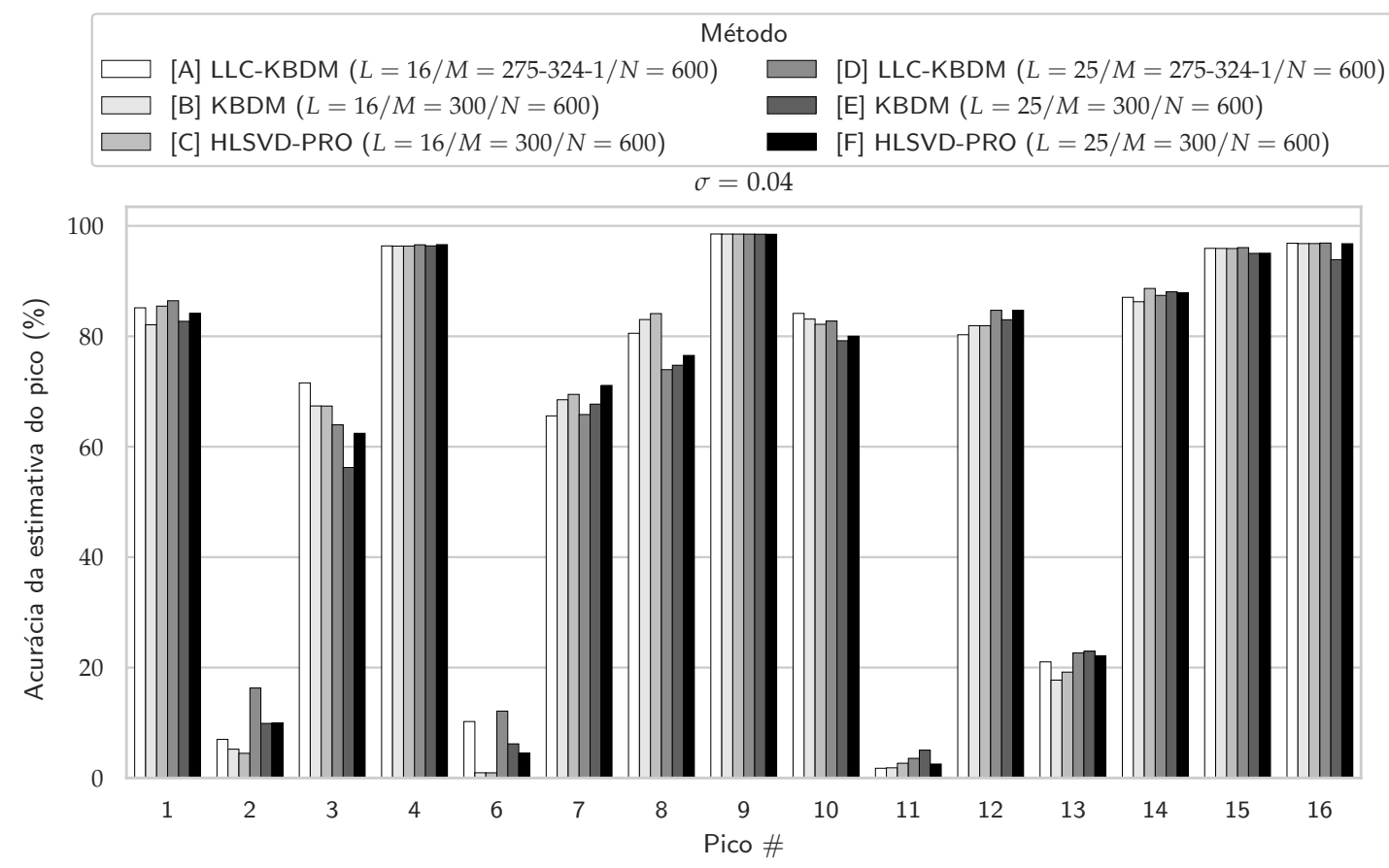

Figura 5.9 - Acurácia das estimativas bem sucedidas de cada pico para o nível de ruído caracterizado por $\sigma=0.04$ em função do método utilizado. Por não ter sido detectado em nenhum dos casos, o pico \#5 foi omitido do gráfico.

Fonte: Elaborada pelo autor.

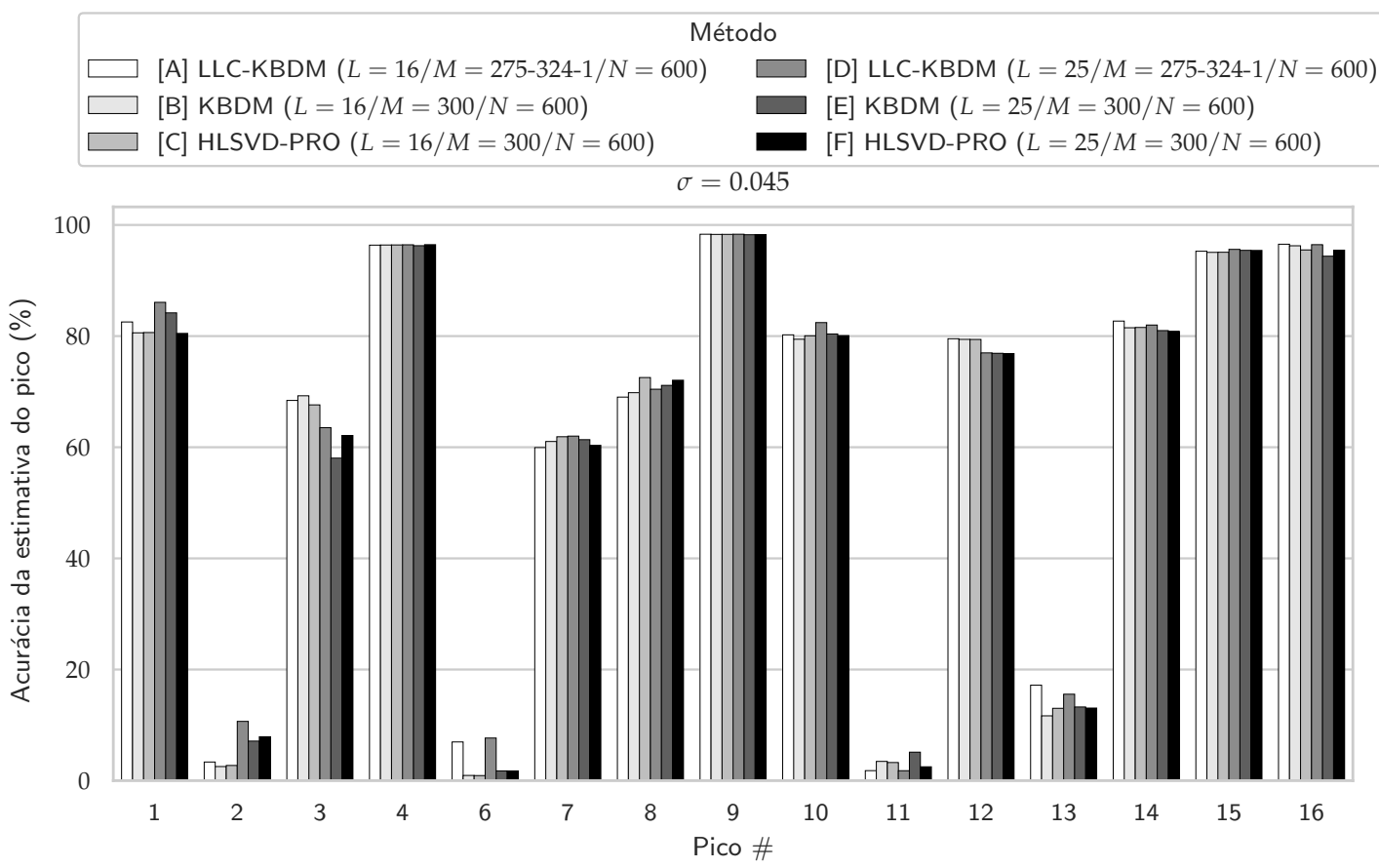

Figura 5.10 - Acurácia das estimativas bem sucedidas de cada pico para o nível de ruído caracterizado por $\sigma=0.045$ em função do método utilizado. Por não ter sido detectado em nenhum dos casos, o pico \#5 foi omitido do gráfico.

Fonte: Elaborada pelo autor. 


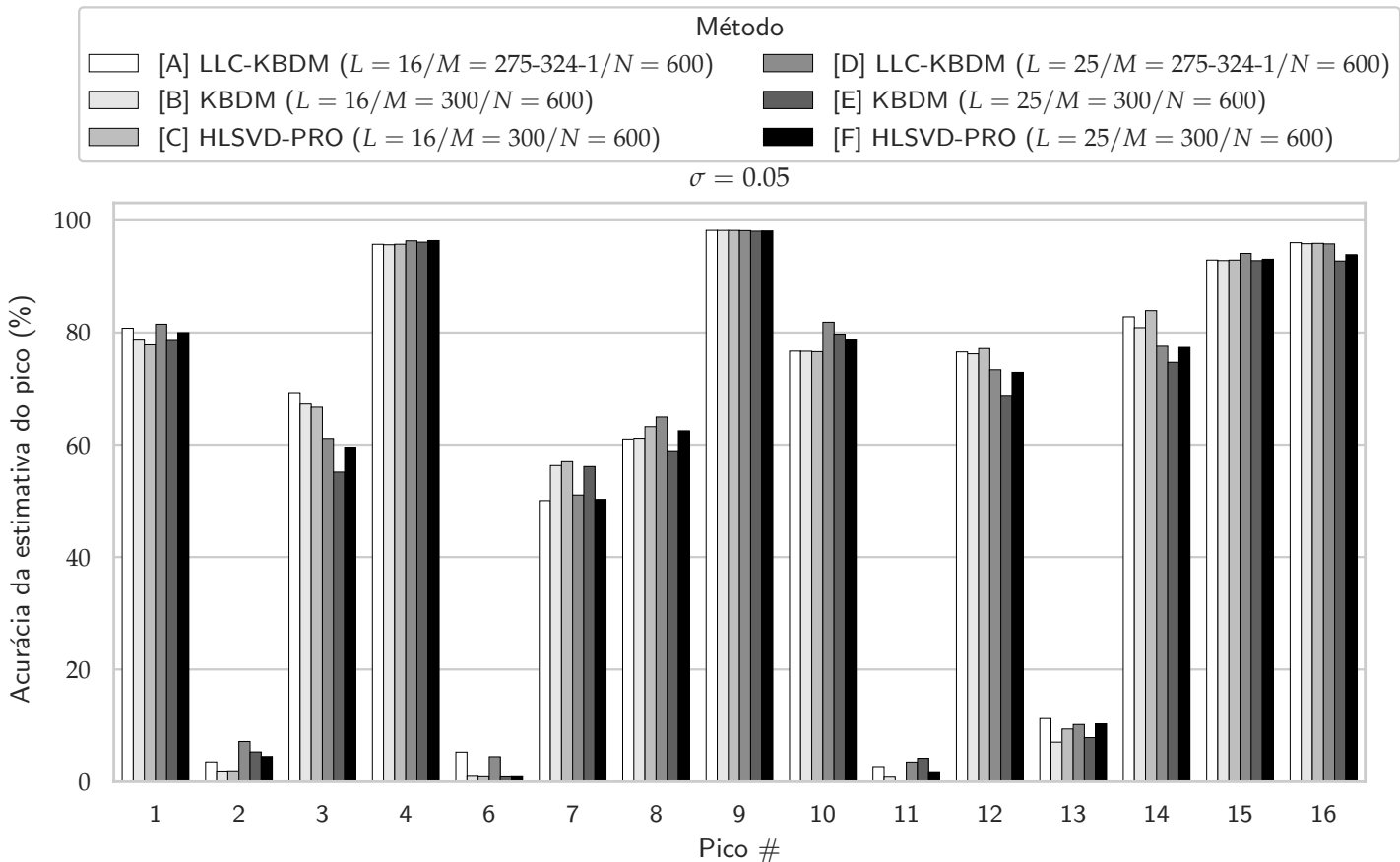

Figura 5.11 - Acurácia das estimativas bem sucedidas de cada pico para o nível de ruído caracterizado por $\sigma=0.025$ em função do método utilizado. Por não ter sido detectado em nenhum dos casos, o pico \#5 foi omitido do gráfico.

Fonte: Elaborada pelo autor.

\subsection{Espectroscopia in vivo do cérebro humano: remoção do sinal da água residual}

\subsubsection{Descrição do estudo}

O procedimento para remoção do sinal residual da água em sinais clínicos de espectroscopia de RM do cérebro humano será demonstrado. Foram utilizados 14 espectros adquiridos retrospectivamente em um equipamento Philips 3T Achieva equipado com gradientes capazes de gerar amplitudes de $80 \mathrm{mT} / \mathrm{m}$ e $200 \mathrm{mT} / \mathrm{m} / \mathrm{ms}$ de rampa de subida. A aquisição foi feita na região do giro do cíngulo posterior utilizando um sequência PRESS de voxel único (25mmx $25 m m x 25 m m)$ com os seguintes parâmetros: TE/TR $=31 / 2000 \mathrm{~ms}, 2048$ pontos, $2 k H z$ de largura de banda na recepção e 96 médias. Para a obtenção dos espectros, um ajuste automático do campo local e a supressão do sinal água foram realizadas pelo scanner.

Foram utilizados os métodos LLC-KBDM, KBDM e HLSVD-PRO, com $N=2000$ (número de pontos utilizados do sinal), $M=1000$ (número de linhas das matrizes de sobreposição 
com simetria de Hankel) e $J=25$ (número de valores singulares considerados após o truncamento das matrizes) para o KBDM e HLSVD-PRO. As amostragens do KBDM para o LLC-KBDM em cada iteração foram realizadas com $M=975,976, \ldots, 1024$ ( $W=50$ amostras), $J=25$. O número máximo de iterações para o LLC-KBDM utilizado foi $i_{\max }=5$.

A região onde o sinal da água foi considerado presente vai de aproximadamente $4.17 \mathrm{ppm}$ até $6.22 \mathrm{ppm}$. Após a supressão, deseja-se que toda a contribuição espectral dentro deste intervalo seja removida.

Em função da natureza dos dados, não há como realizar medidas diretas para julgar a qualidade do ajuste. No entanto, a obtenção de um resíduo onde não exista nenhuma componente espectral com frequência central na região onde houve a supressão da água é indicação de que o ajuste foi realizado com sucesso.

Idealmente, isto ocorre quando há apenas ruído no resíduo calculado a partir do espectro estimado em toda a banda espectral. É necessário utilizar a estimativa total do sinal, ainda que as linhas obtidas fora da região da água sejam descartadas. Caso contrário, os picos do sinais de formatos mais largos com frequências centrais fora da região da água adicionariam contribuições na região onde houve supressão, dificultando ainda mais a avaliação do resultado.

Embora os formatos das linhas obtidas na região dos metabólitos não sejam os mais adequados em função do alargamento existente nesses picos, o ajuste total do sinal é suficiente o bastante para o propósito apresentado.

Sendo assim, a seguinte estratégia foi utilizada para a avaliação dos métodos:

1. O ajuste do sinal é realizado através do KBDM, LLC-KBDM e HLSVD-PRO;

2. Um teste estatístico para verificar se as amostras contidas na região espectral do resíduo onde o sinal da água será suprimido seguem uma distribuição normal é realizado através da função scipy. stats. normaltest da SciPy. (99) Este teste utiliza a curtose e a obliquidade da distribuição. A hipótese nula $H_{0}$ afirma que as amostras seguem distribuição a normal; a hipótese alternativa, que rejeita $H_{0}$, será denominada $H_{1}$. Três níveis de significância $(\alpha=0.05,0.01,0.001)$ serão considerados na pontuação do número de rejeições da hipótese nula para os 14 espectros utilizando cada método;

3. O sinal após a supressão é calculado a partir da subtração do sinal parcial com linhas estimadas de frequência central no interior do intervalo espectral da água;

4. Visualizações gráficas das distribuições do resíduo na região da água e dos espectros após a remoção do sinal residual serão apresentadas. 


\subsubsection{Resultados e discussões}

As Figuras 5.12-5.25 mostram os resultados obtidos para os 14 espectros estudados. Na primeira linha de cada figura, A), são mostrados, de cima para baixo, o sinal processado contendo sinal da água residual na região demarcada através das linhas verticais tracejadas em vermelho; e os espectros após a realização do procedimento de supressão para métodos LLC-KBDM, KBDM e HLSVD-PRO. Na segunda linha, B), o resíduo entre as estimativas obtidas pelos métodos na região espectral onde a supressão ocorre é mostrado. Na última linha, as colunas C) e D) mostram, respectivamente, a estimativa da distribuição e o diagrama de caixa do resíduo nesta mesma região.

A Tabela 5.1 mostra todas as medidas estatísticas utilizadas para avaliação da qualidade da distribuição do sinal residual para cada espectro estimado pelos métodos KBDM, LLC-KBDM e HLSVD-PRO na região espectral onde a supressão da água é realizada. A Tabela 5.2 mostra o somatório de hipóteses nulas não rejeitas que será, portanto, a métrica utilizada na comparação entre os métodos.

De maneira geral, todos os métodos cumpriram com o papel da remoção das contribuições mais acentuadas do sinal da água em cada um dos espectros. No entanto, o LLC-KBDM se destacou nos resultados em relação à qualidade dos resíduos tanto graficamente, quanto através do teste de hipótese em relação à normalidade da distribuição do resíduo. Outra característica do LLC-KBDM é produzir resíduos com desvio padrão sistematicamente menor do que o KBDM e o HLSVD-PRO.

O KBDM e o HLSVD-PRO desempenharam de maneira semelhante no estudo realizado. 
Tabela 5.1 - Teste de hipóteses para a distribuição do resíduo na região onde o sinal da água residual foi suprimido para três níveis de significância $(\alpha=0.001,0.01,0.05)$. A estatística $k^{2}$ combina a curtose e a obliquidade da distribuição testada.

\begin{tabular}{|c|c|c|c|c|c|c|c|c|}
\hline Espectro & Método & Desvio Padrão & Média & Estatística $\left(k^{2}\right)$ & valor-p & $\begin{array}{l}H_{0} \text { aceita } \\
(\alpha=0.001)\end{array}$ & $\begin{array}{l}H_{0} \text { aceita } \\
(\alpha=0.01)\end{array}$ & $\begin{array}{l}H_{0} \text { aceita } \\
(\alpha=0.05)\end{array}$ \\
\hline \multirow[t]{3}{*}{1} & HLSVD-PRO & 0.000207 & 0.000113 & 12.405225 & 0.002024 & Sim & Não & Não \\
\hline & KBDM & 0.000206 & 0.000105 & 12.122747 & 0.002331 & Sim & Não & Não \\
\hline & LLC-KBDM & 0.000174 & 0.000042 & 8.228390 & 0.016339 & Sim & Sim & Não \\
\hline \multirow[t]{3}{*}{2} & HLSVD-PRO & 0.000212 & 0.000067 & 0.520232 & 0.770962 & Sim & Sim & Sim \\
\hline & KBDM & 0.000210 & 0.000067 & 0.406201 & 0.816196 & Sim & Sim & Sim \\
\hline & LLC-KBDM & 0.000162 & 0.000019 & 4.041542 & 0.132553 & Sim & Sim & Sim \\
\hline \multirow[t]{3}{*}{3} & HLSVD-PRO & 0.000197 & -0.000001 & 4.895002 & 0.086510 & Sim & Sim & Sim \\
\hline & KBDM & 0.000196 & 0.000003 & 3.626241 & 0.163144 & Sim & Sim & Sim \\
\hline & LLC-KBDM & 0.000159 & -0.000004 & 0.163732 & 0.921396 & Sim & Sim & Sim \\
\hline \multirow[t]{3}{*}{4} & HLSVD-PRO & 0.000218 & 0.000152 & 10.892776 & 0.004312 & Sim & Não & Não \\
\hline & KBDM & 0.000218 & 0.000159 & 11.968261 & 0.002518 & Sim & Não & Não \\
\hline & LLC-KBDM & 0.000270 & 0.000052 & 10.815166 & 0.004482 & Sim & Não & Não \\
\hline \multirow[t]{3}{*}{5} & HLSVD-PRO & 0.000277 & 0.000057 & 16.639590 & 0.000244 & Não & Não & Não \\
\hline & KBDM & 0.000277 & 0.000053 & 15.712801 & 0.000387 & Não & Não & Não \\
\hline & LLC-KBDM & 0.000231 & 0.000065 & 5.618983 & 0.060236 & Sim & Sim & Sim \\
\hline \multirow[t]{3}{*}{6} & HLSVD-PRO & 0.000150 & 0.000013 & 7.260010 & 0.026516 & Sim & Sim & Não \\
\hline & KBDM & 0.000149 & 0.000013 & 6.661428 & 0.035768 & Sim & Sim & Não \\
\hline & LLC-KBDM & 0.000141 & 0.000004 & 4.259253 & 0.118882 & Sim & Sim & Sim \\
\hline \multirow[t]{3}{*}{7} & HLSVD-PRO & 0.000290 & -0.000007 & 8.420444 & 0.014843 & Sim & Sim & Não \\
\hline & KBDM & 0.000289 & -0.000012 & 8.416041 & 0.014876 & Sim & Sim & Não \\
\hline & LLC-KBDM & 0.000179 & 0.000005 & 1.362366 & 0.506018 & Sim & Sim & Sim \\
\hline \multirow[t]{3}{*}{8} & HLSVD-PRO & 0.000331 & -0.000014 & 2.794825 & 0.247236 & Sim & Sim & Sim \\
\hline & KBDM & 0.000338 & -0.000019 & 6.882865 & 0.032019 & Sim & Sim & Não \\
\hline & LLC-KBDM & 0.000290 & -0.000153 & 17.806528 & 0.000136 & Não & Não & Não \\
\hline \multirow[t]{3}{*}{9} & HLSVD-PRO & 0.000207 & 0.000030 & 0.433770 & 0.805023 & Sim & Sim & Sim \\
\hline & KBDM & 0.000207 & 0.000027 & 0.417654 & 0.811536 & Sim & Sim & Sim \\
\hline & LLC-KBDM & 0.000158 & 0.000013 & 0.548280 & 0.760226 & Sim & Sim & Sim \\
\hline \multirow[t]{3}{*}{10} & HLSVD-PRO & 0.000284 & 0.000107 & 14.680749 & 0.000649 & Não & Não & Não \\
\hline & KBDM & 0.000278 & 0.000095 & 13.507518 & 0.001166 & Sim & Não & Não \\
\hline & LLC-KBDM & 0.000236 & 0.000066 & 6.734909 & 0.034477 & Sim & Sim & Não \\
\hline \multirow[t]{3}{*}{11} & HLSVD-PRO & 0.000180 & 0.000022 & 0.350197 & 0.839374 & Sim & Sim & Sim \\
\hline & KBDM & 0.000181 & 0.000017 & 0.476384 & 0.788051 & Sim & Sim & Sim \\
\hline & LLC-KBDM & 0.000157 & 0.000017 & 1.524872 & 0.466529 & Sim & Sim & Sim \\
\hline \multirow[t]{3}{*}{12} & HLSVD-PRO & 0.000185 & 0.000016 & 0.117275 & 0.943048 & Sim & Sim & Sim \\
\hline & KBDM & 0.000187 & 0.000010 & 0.073704 & 0.963819 & Sim & Sim & Sim \\
\hline & LLC-KBDM & 0.000168 & 0.000013 & 0.122172 & 0.940742 & Sim & Sim & Sim \\
\hline \multirow[t]{3}{*}{13} & HLSVD-PRO & 0.000182 & 0.000043 & 1.258253 & 0.533057 & Sim & Sim & Sim \\
\hline & KBDM & 0.000182 & 0.000044 & 1.350314 & 0.509076 & Sim & Sim & Sim \\
\hline & LLC-KBDM & 0.000147 & 0.000010 & 0.811800 & 0.666377 & Sim & Sim & Sim \\
\hline \multirow[t]{3}{*}{14} & HLSVD-PRO & 0.000240 & 0.000127 & 26.622006 & 0.000002 & Não & Não & Não \\
\hline & KBDM & 0.000235 & 0.000121 & 24.611346 & 0.000005 & Não & Não & Não \\
\hline & LLC-KBDM & 0.000163 & 0.000045 & 4.828591 & 0.089430 & Sim & Sim & Sim \\
\hline
\end{tabular}

Fonte: Elaborada pelo autor.

Tabela 5.2 - Quantidade de espectros onde a hipótese nula não foi rejeitada para cada método em função dos níveis significância $(\alpha)$.

\begin{tabular}{lrrr}
\hline & $H_{0}$ não rejeitada $(\alpha=0.001)$ & $H_{0}$ não rejeitada $(\alpha=0.01)$ & $H_{0}$ não rejeitada $(\alpha=0.05)$ \\
Método & 11 & 9 & 7 \\
\hline HLSVD-PRO & 12 & 9 & 6 \\
KBDM & 13 & 12 & 10 \\
LLC-KBDM & & & \\
\hline
\end{tabular}

Fonte: Elaborada pelo autor. 
A) Espectros obtidos após supressão do sinal residual da água

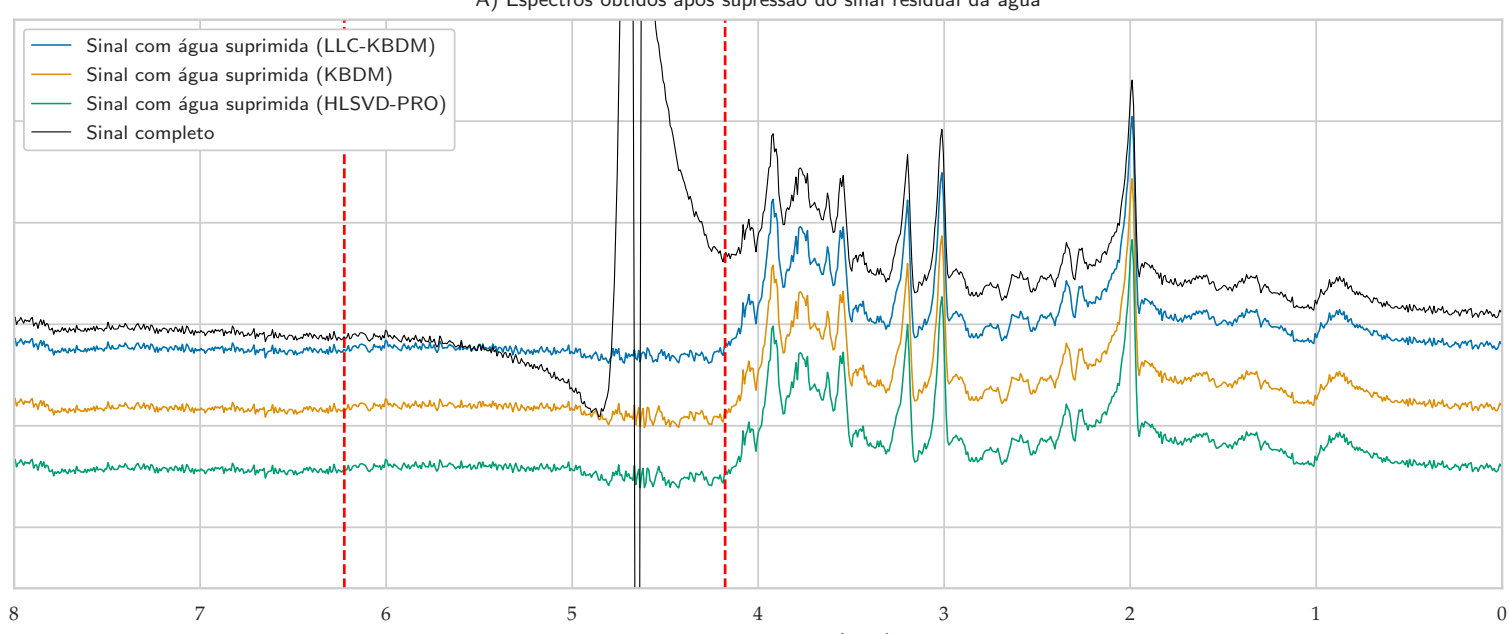

Deslocamento químico $(\mathrm{ppm})$

B) Resíduo do sinal estimado na região suprimida

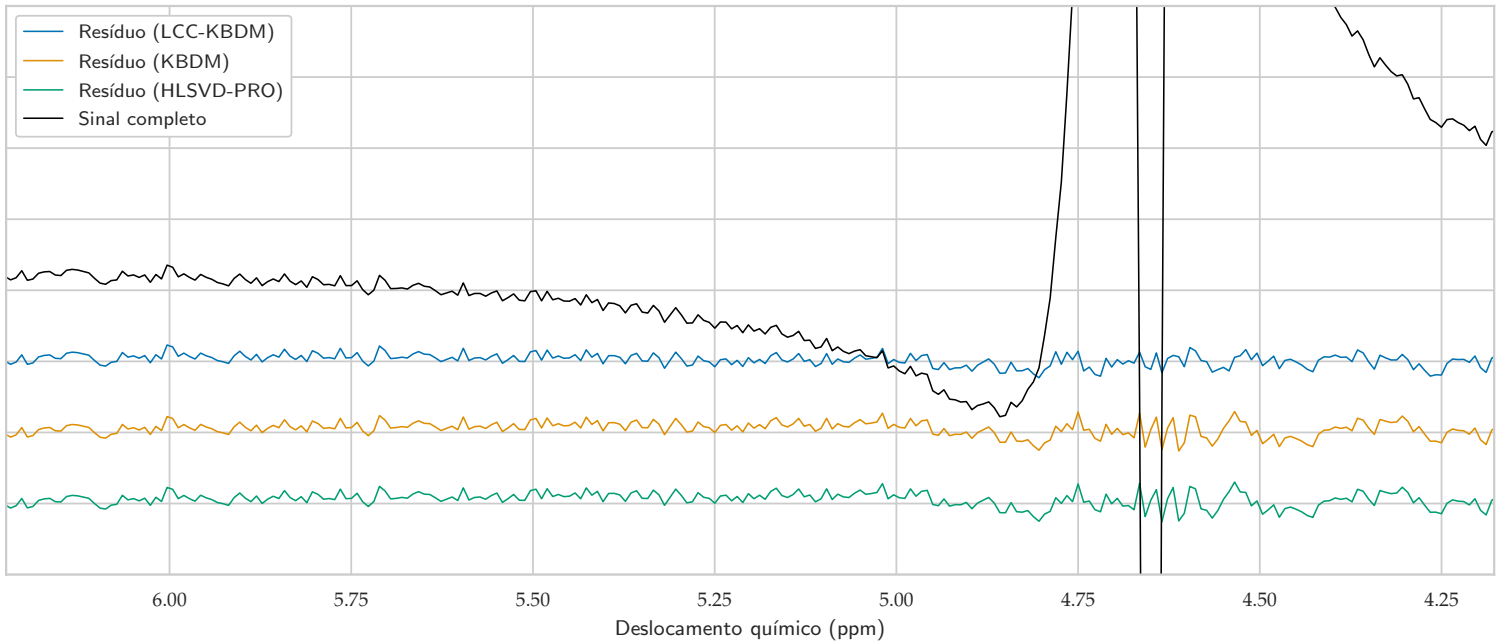

C) Estimativa da distribuição do resíduo na região suprimida
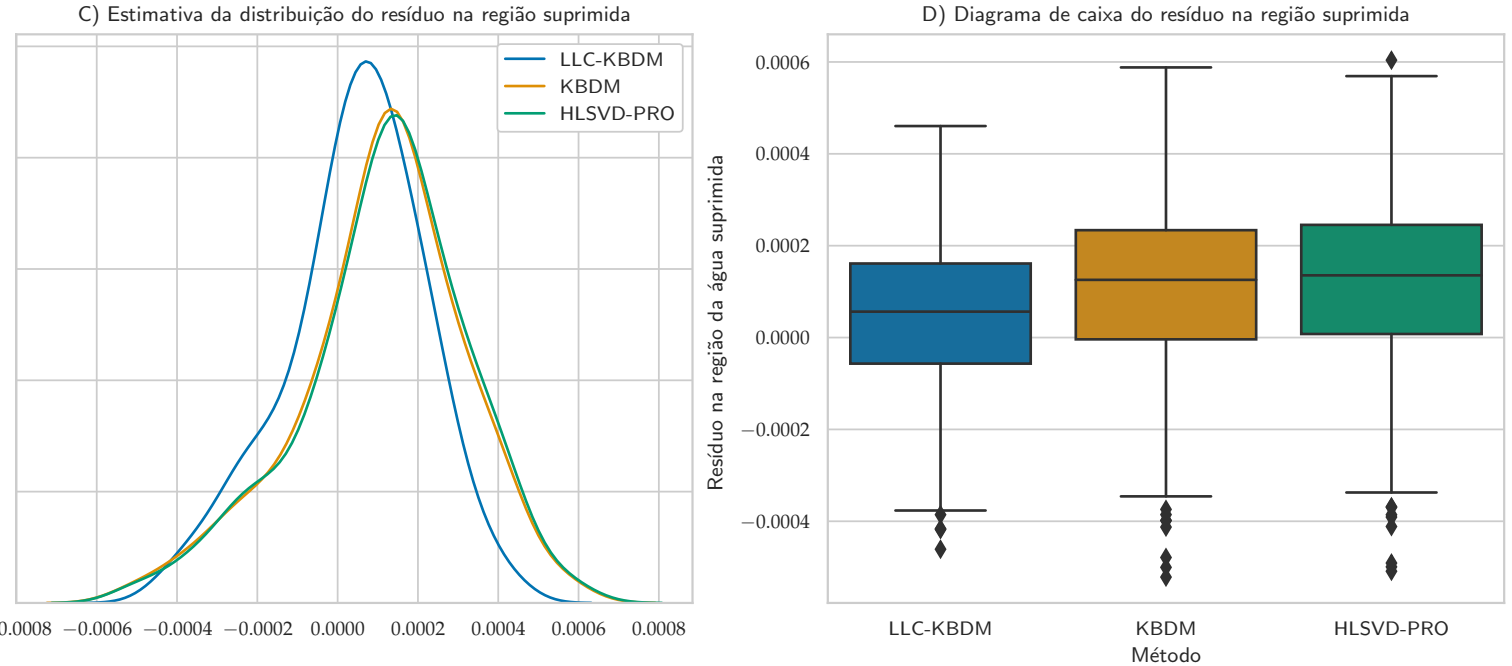

Figura 5.12 - Resultados obtidos para o espectro \#1. As linhas verticais tracejadas em A) denotam a região onde a água foi suprimida. Os pontos pretos nos diagramas de caixa em D) indicam a presença de outliers, caracterizado pela presença de sinal remanescente na região após o procedimento. O sinal completo denota o espectro total, antes da remoção da água.

Fonte: Elaborada pelo autor. 
A) Espectros obtidos após supressão do sinal residual da água

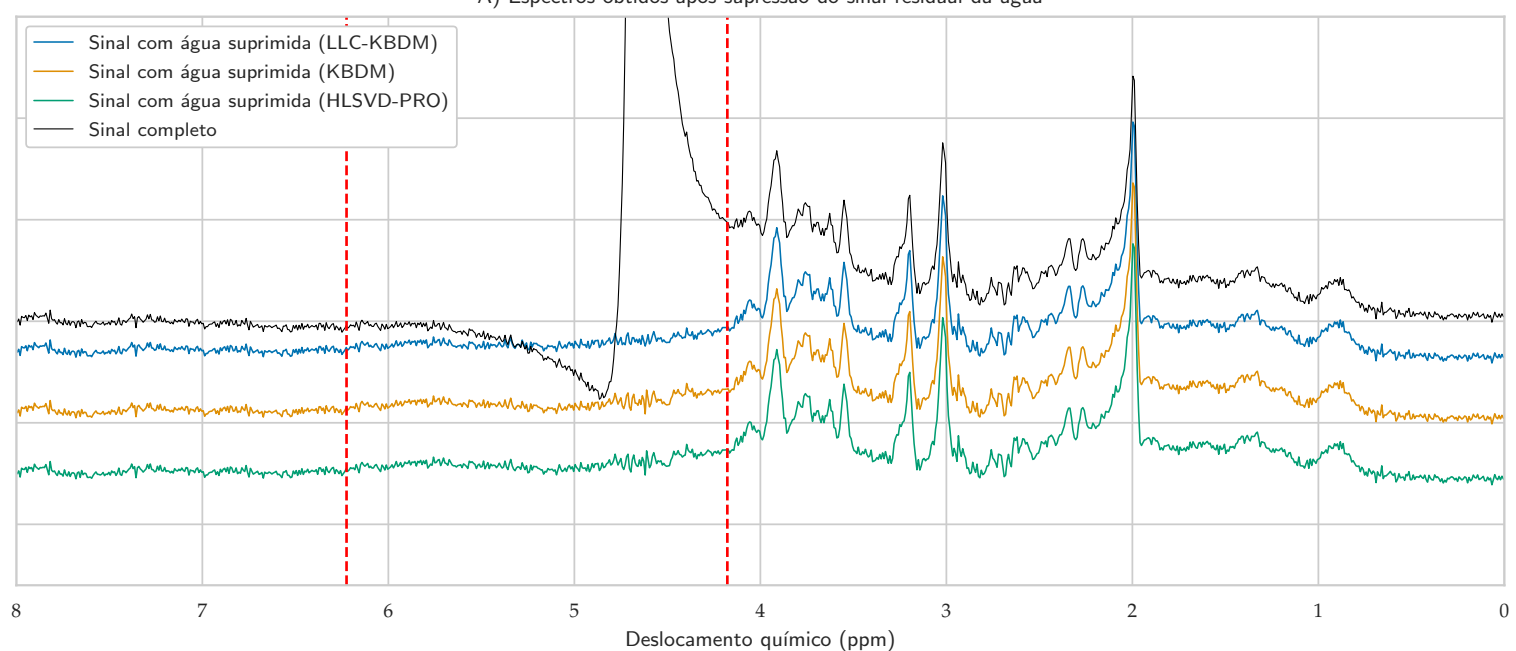

B) Resíduo do sinal estimado na região suprimida

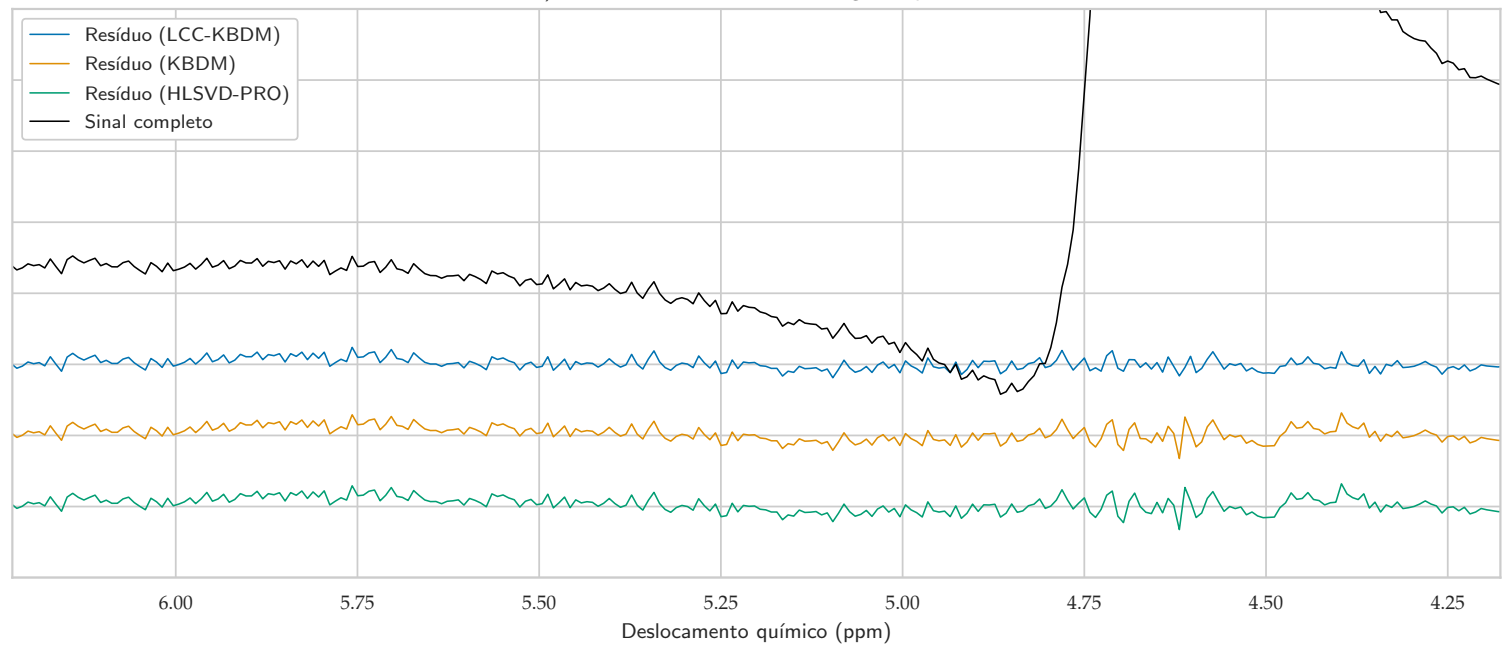

C) Estimativa da distribuição do resíduo na região suprimida

D) Diagrama de caixa do resíduo na região suprimida
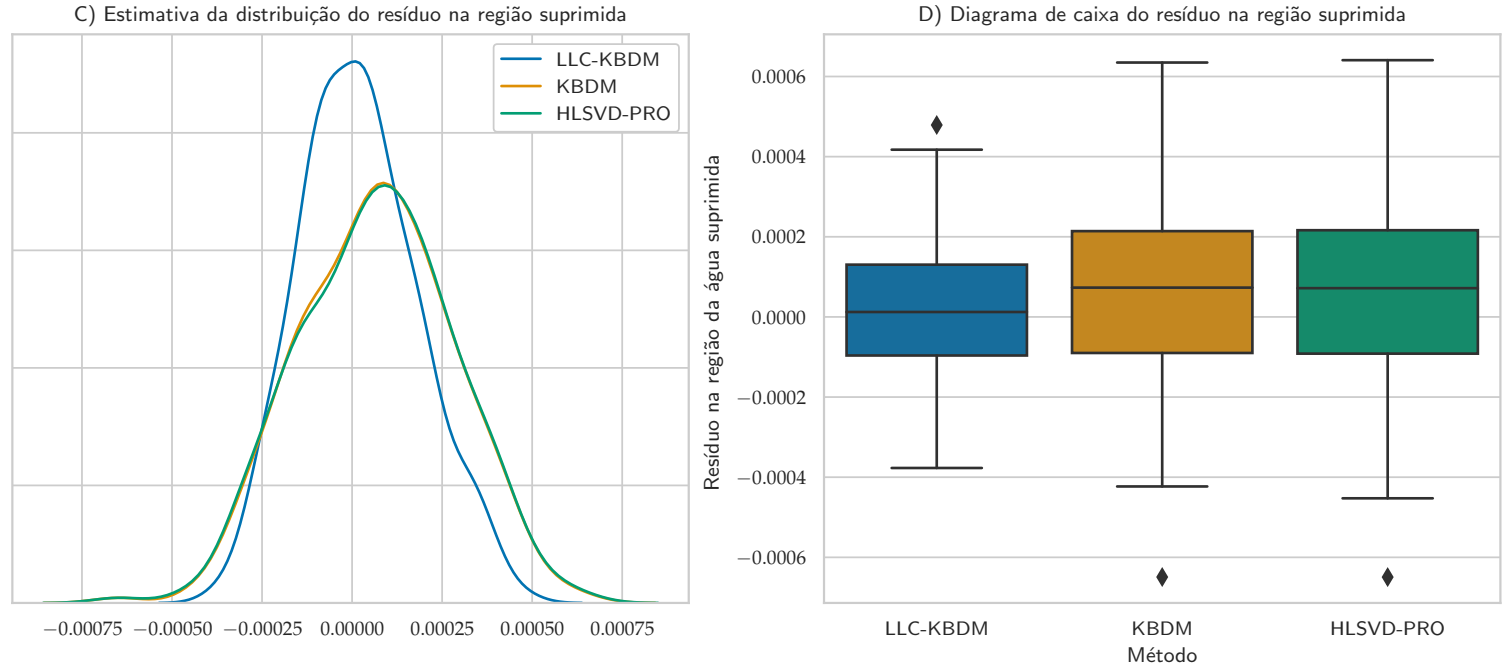

Figura 5.13 - Resultados obtidos para o espectro \#2. As linhas verticais tracejadas em A) denotam a região onde a água foi suprimida. Os pontos pretos nos diagramas de caixa em D) indicam a presença de outliers, caracterizado pela presença de sinal remanescente na região após o procedimento. O sinal completo denota o espectro total, antes da remoção da água.

Fonte: Elaborada pelo autor. 
A) Espectros obtidos após supressão do sinal residual da água

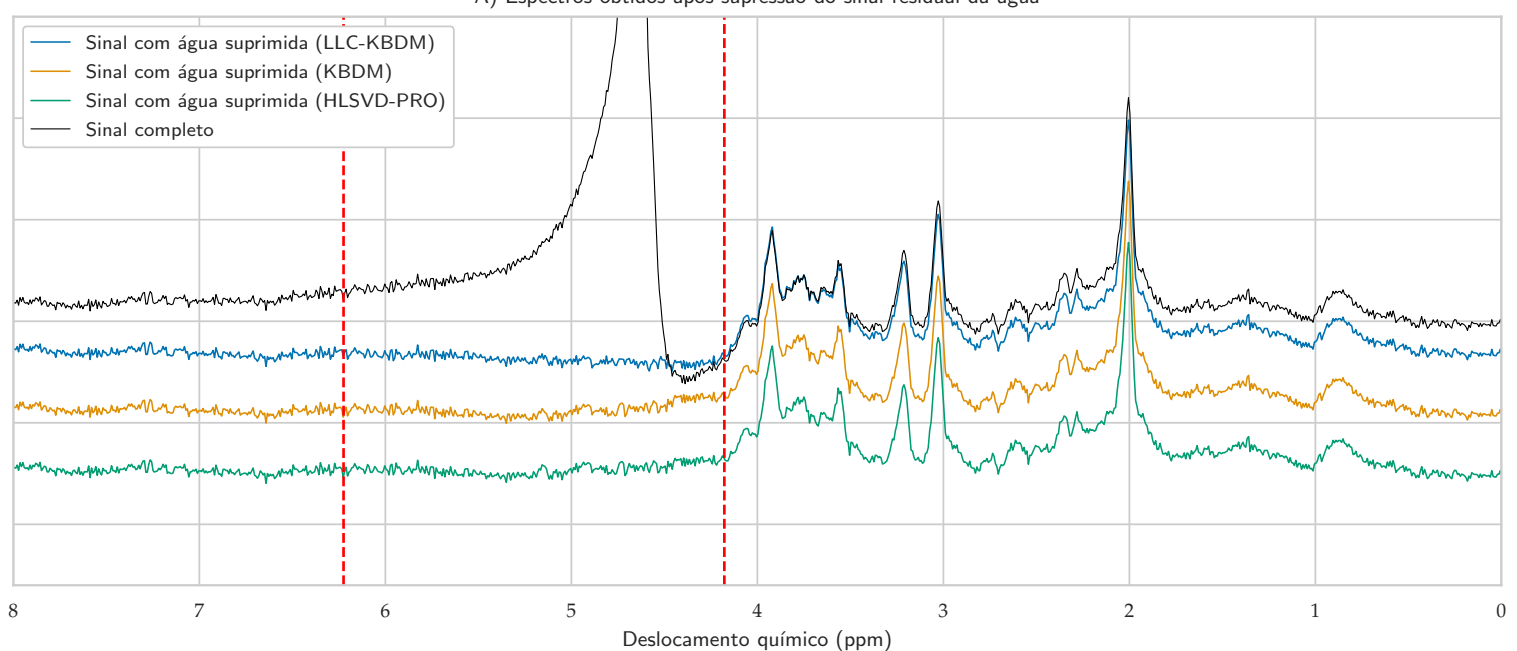

B) Resíduo do sinal estimado na região suprimida

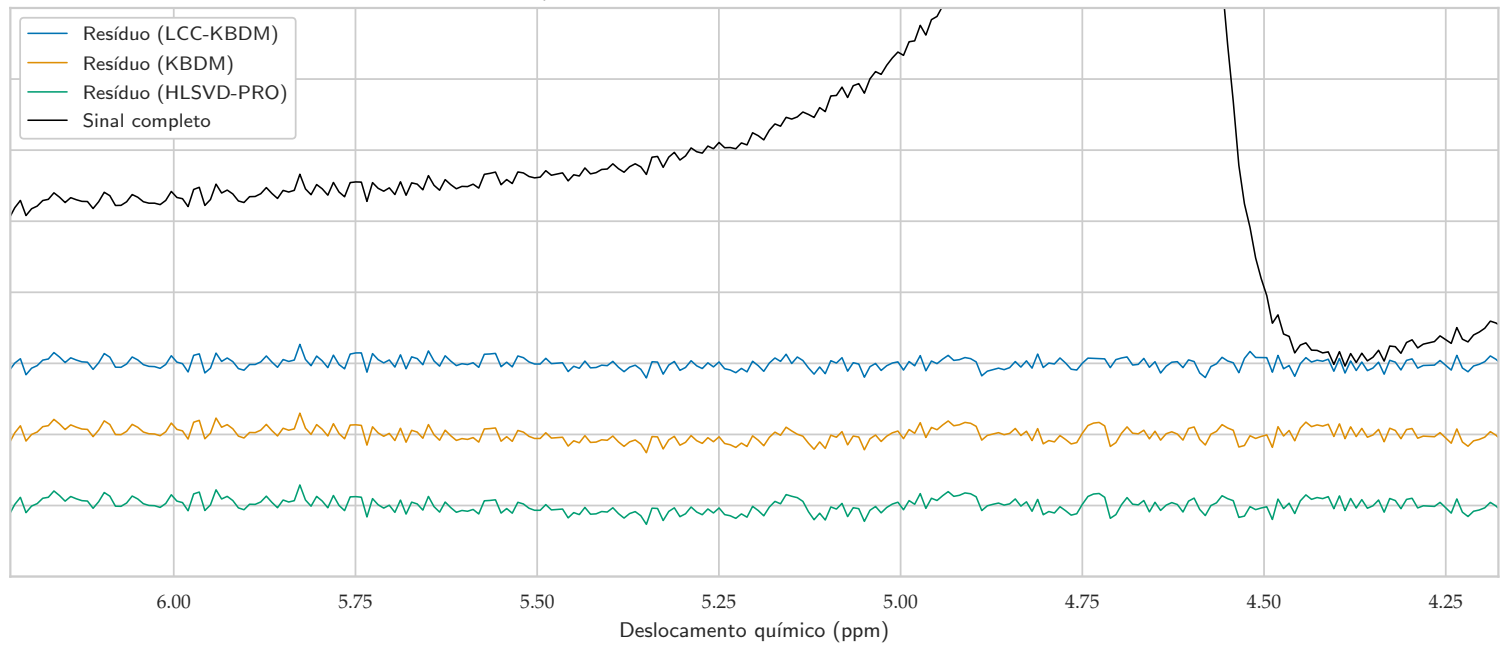

C) Estimativa da distribuição do resíduo na região suprimida

D) Diagrama de caixa do resíduo na região suprimida
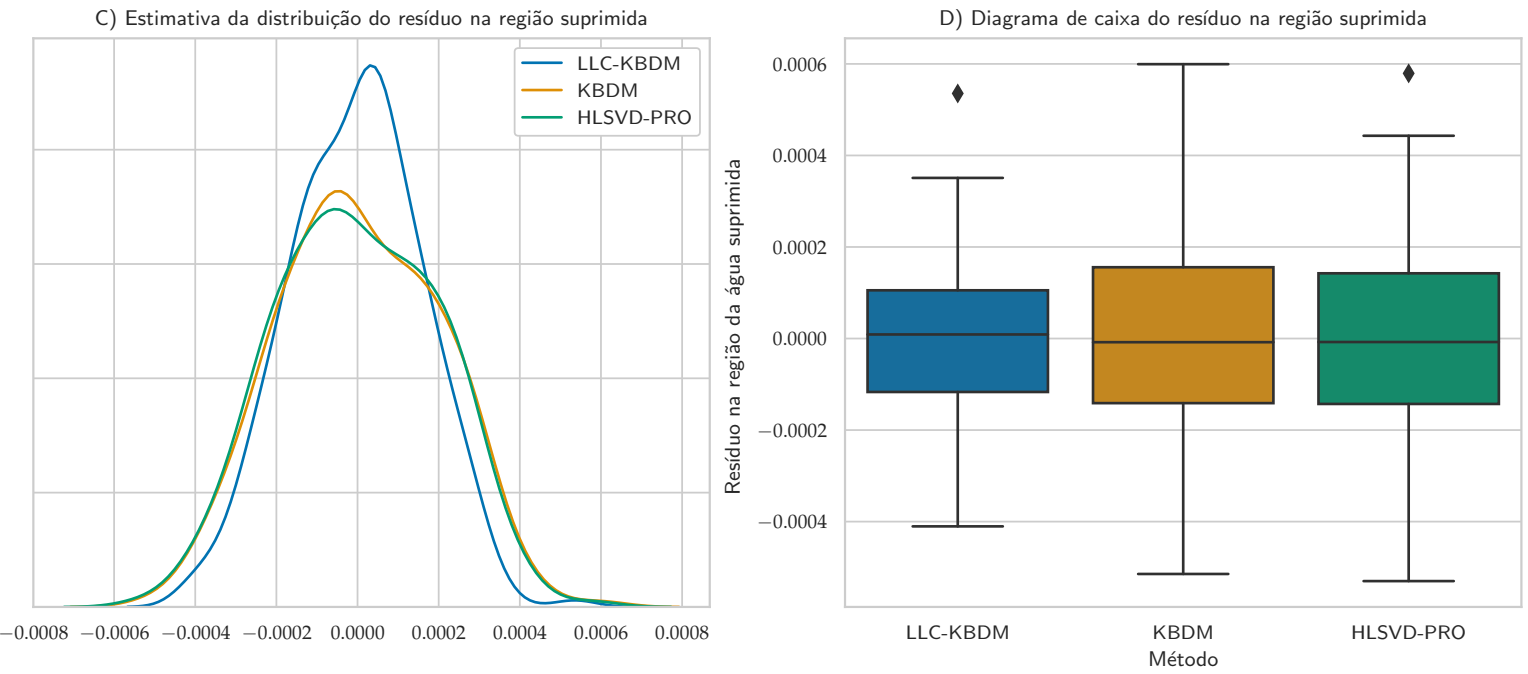

Figura 5.14 - Resultados obtidos para o espectro \#3. As linhas verticais tracejadas em A) denotam a região onde a água foi suprimida. Os pontos pretos nos diagramas de caixa em D) indicam a presença de outliers, caracterizado pela presença de sinal remanescente na região após o procedimento. O sinal completo denota o espectro total, antes da remoção da água.

Fonte: Elaborada pelo autor. 
A) Espectros obtidos após supressão do sinal residual da água

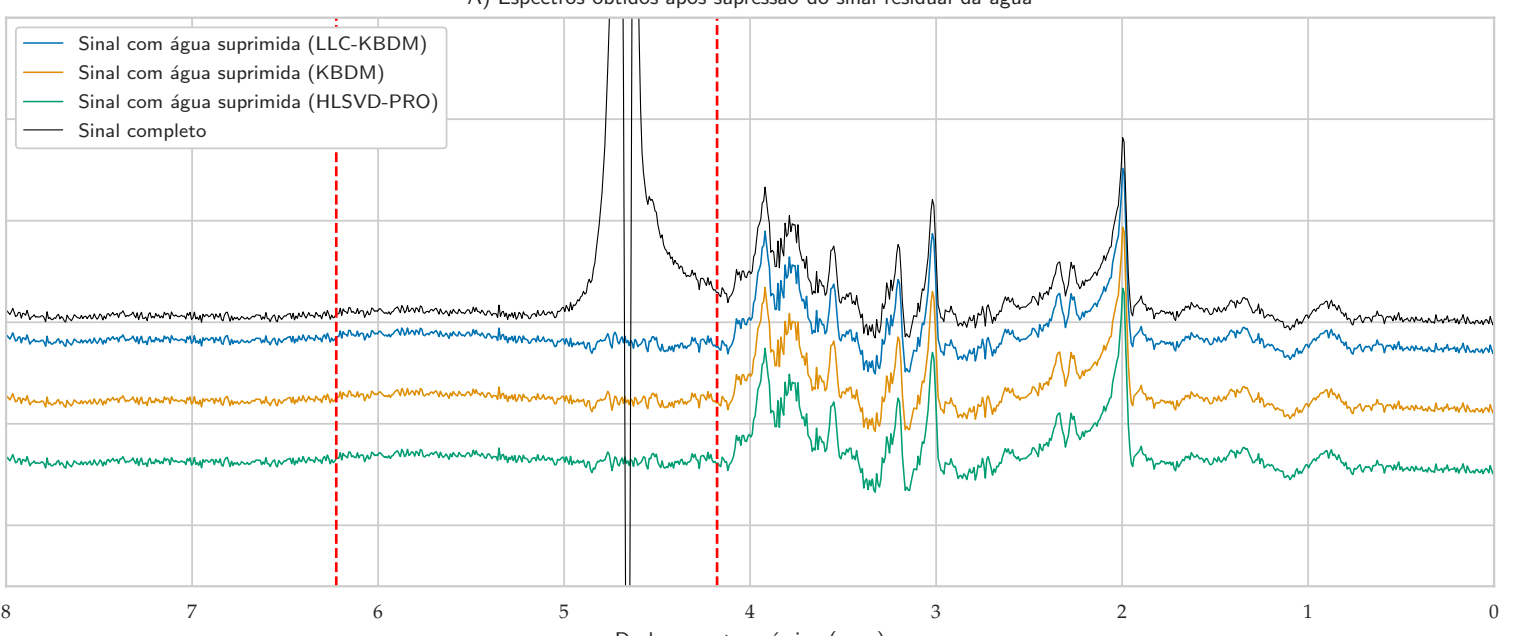

B) Resíduo do sinal estimado na região suprimida

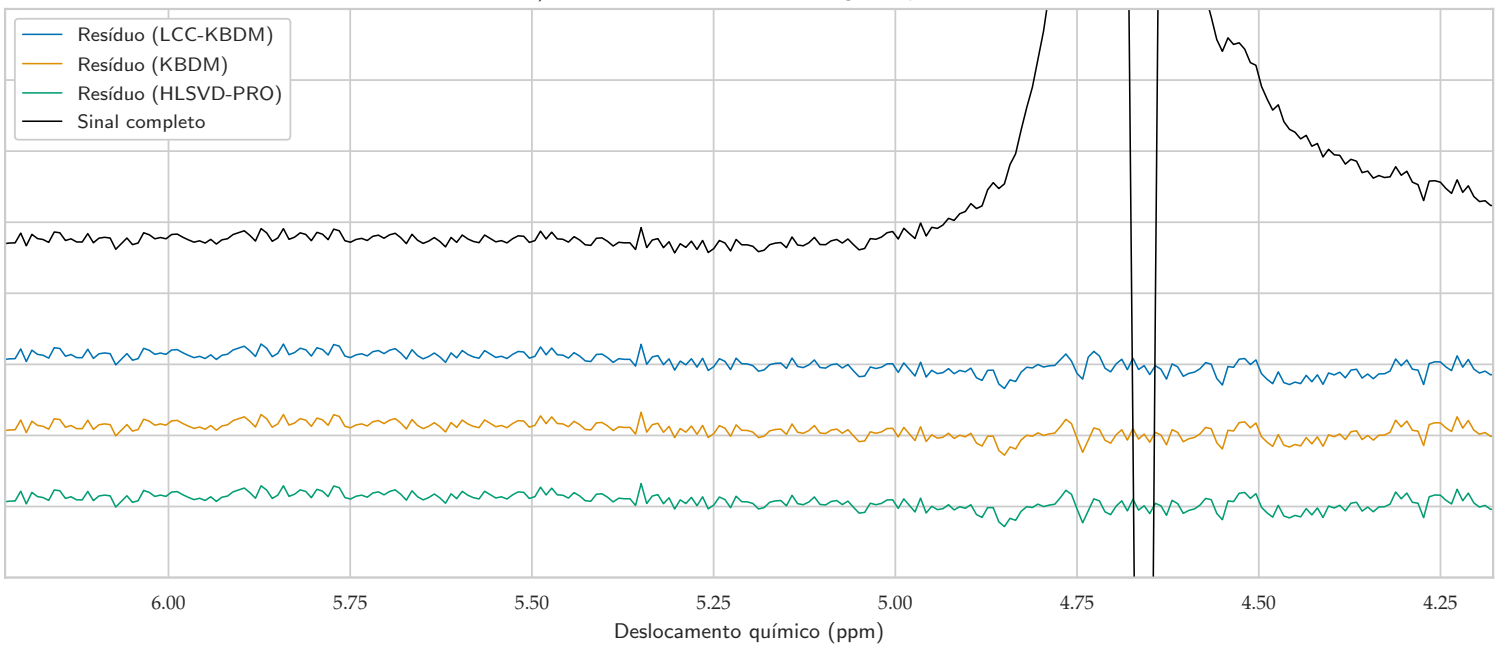

C) Estimativa da distribuição do resíduo na região suprimida

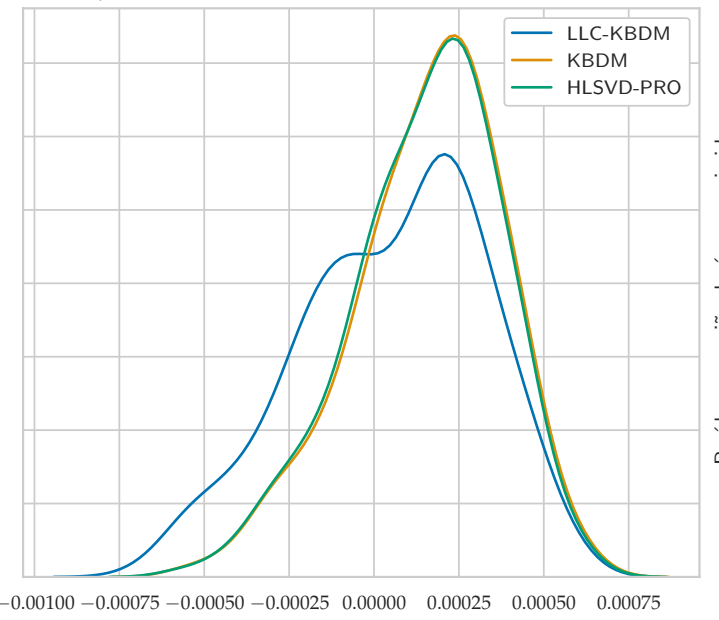

D) Diagrama de caixa do resíduo na região suprimida

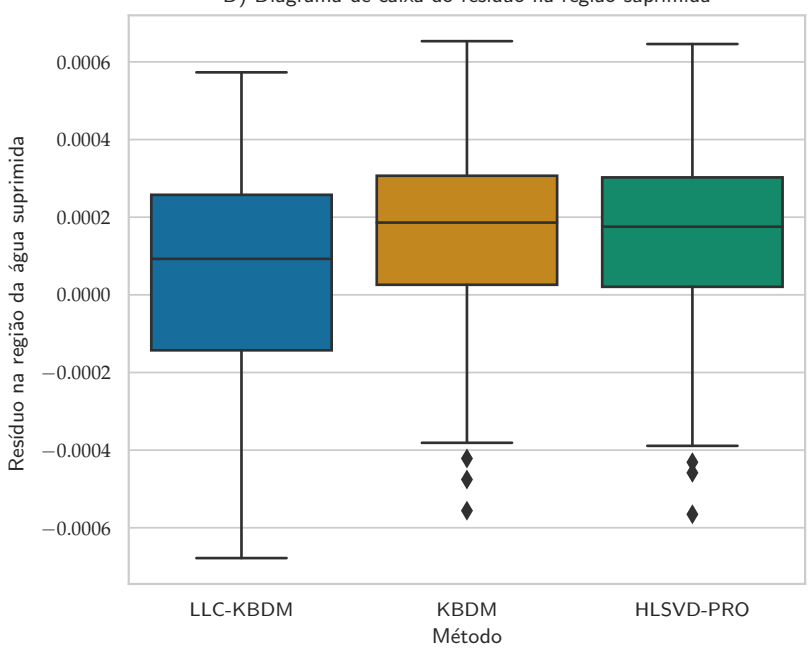

Figura 5.15 - Resultados obtidos para o espectro \#4. As linhas verticais tracejadas em A) denotam a região onde a água foi suprimida. Os pontos pretos nos diagramas de caixa em D) indicam a presença de outliers, caracterizado pela presença de sinal remanescente na região após o procedimento. O sinal completo denota o espectro total, antes da remoção da água.

Fonte: Elaborada pelo autor. 
A) Espectros obtidos após supressão do sinal residual da água

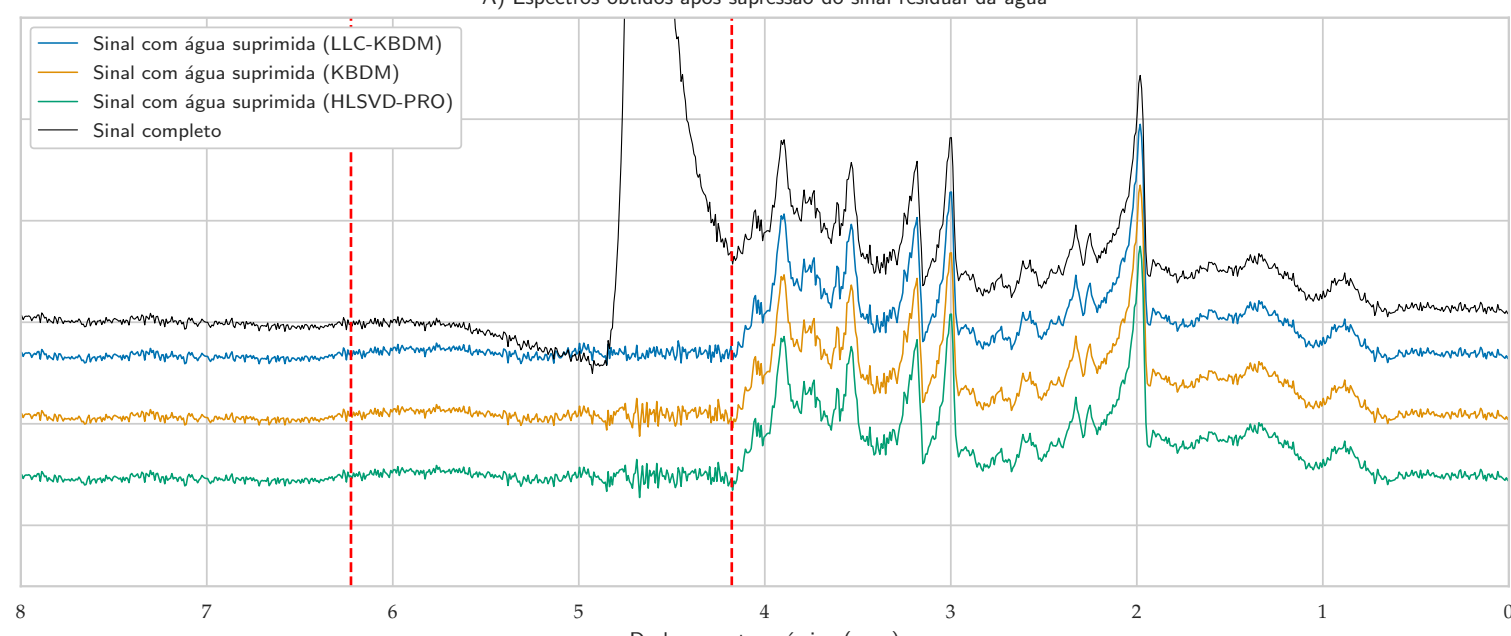

B) Resíduo do sinal estimado na região suprimida

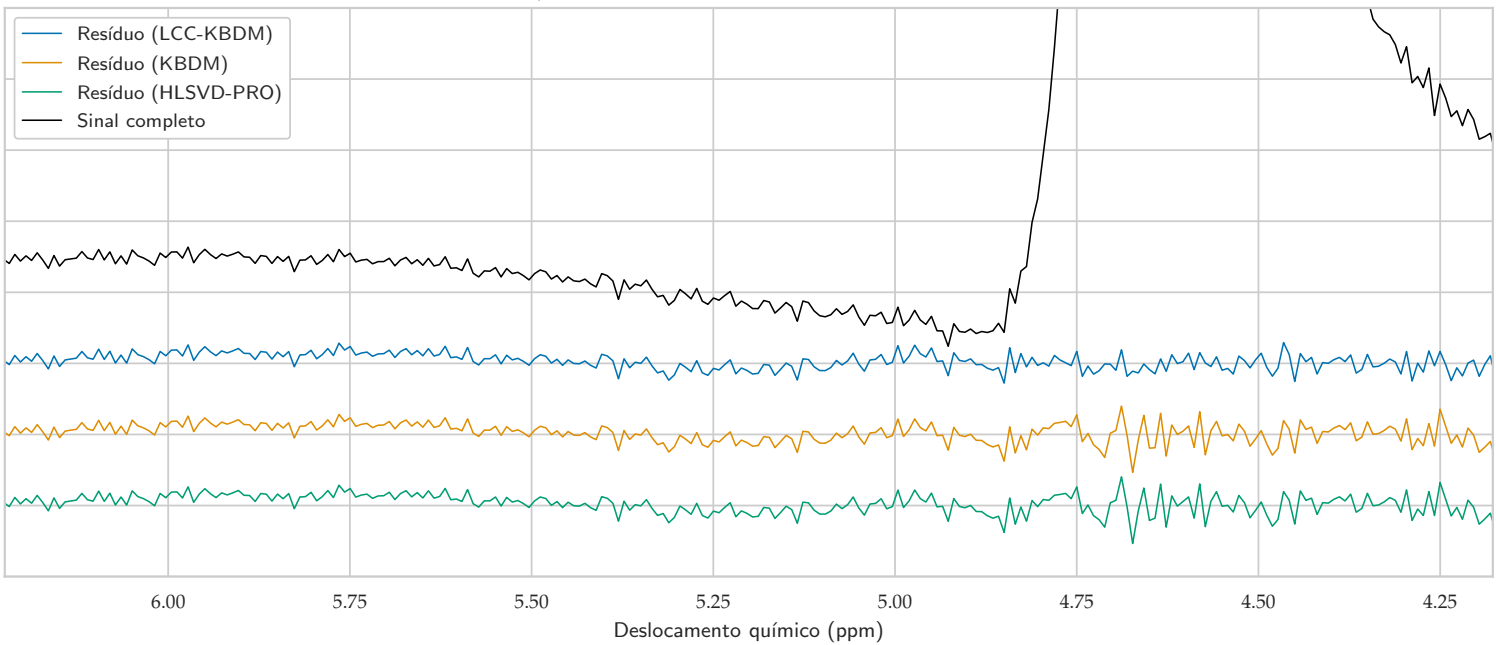

C) Estimativa da distribuição do resíduo na região suprimida
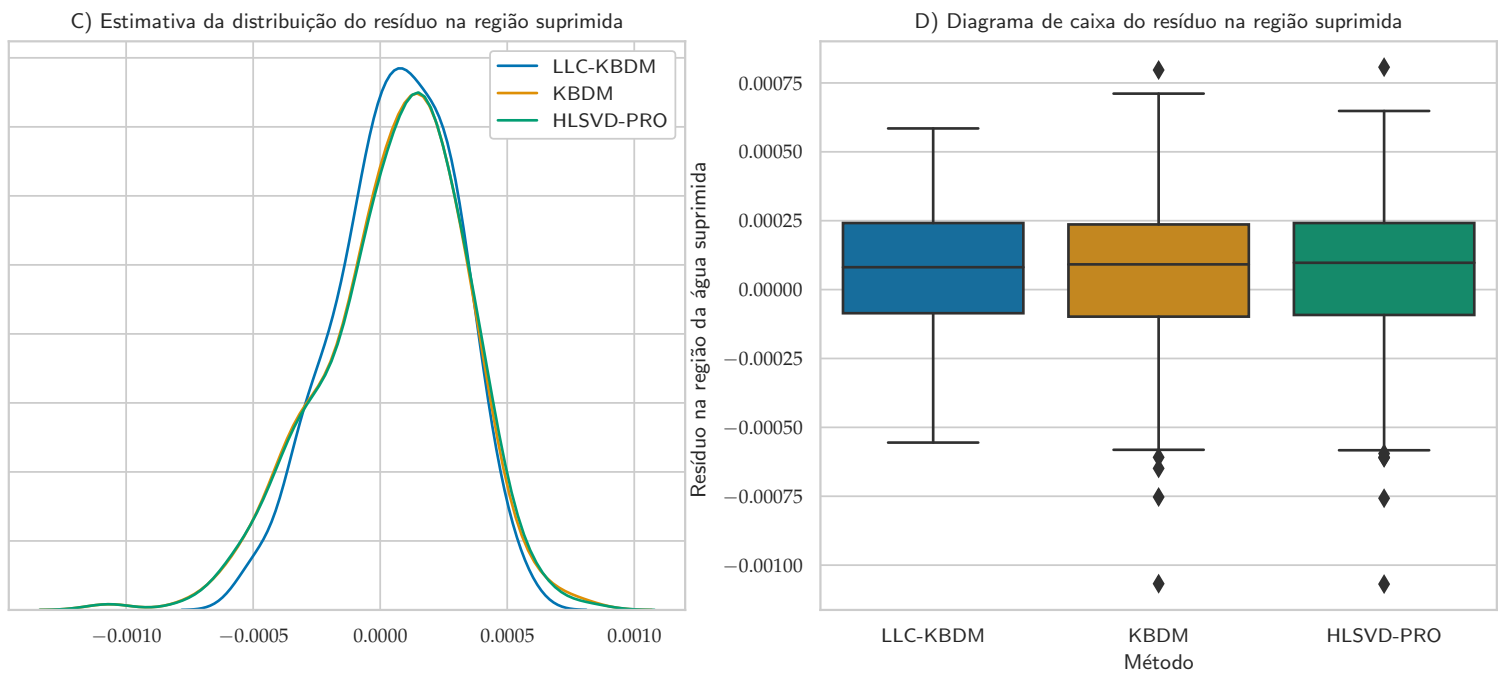

Figura 5.16 - Resultados obtidos para o espectro \#5. As linhas verticais tracejadas em A) denotam a região onde a água foi suprimida. Os pontos pretos nos diagramas de caixa em D) indicam a presença de outliers, caracterizado pela presença de sinal remanescente na região após o procedimento. O sinal completo denota o espectro total, antes da remoção da água.

Fonte: Elaborada pelo autor. 
A) Espectros obtidos após supressão do sinal residual da água

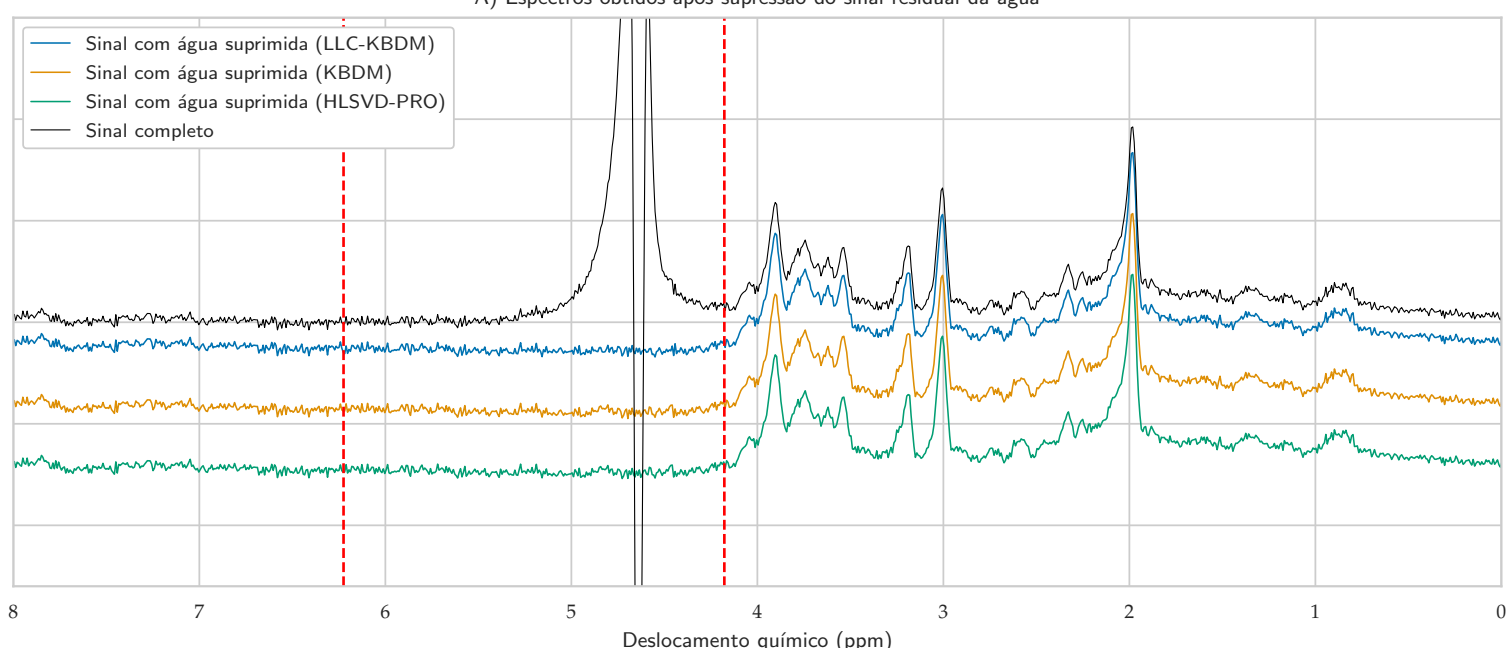

B) Resíduo do sinal estimado na região suprimida

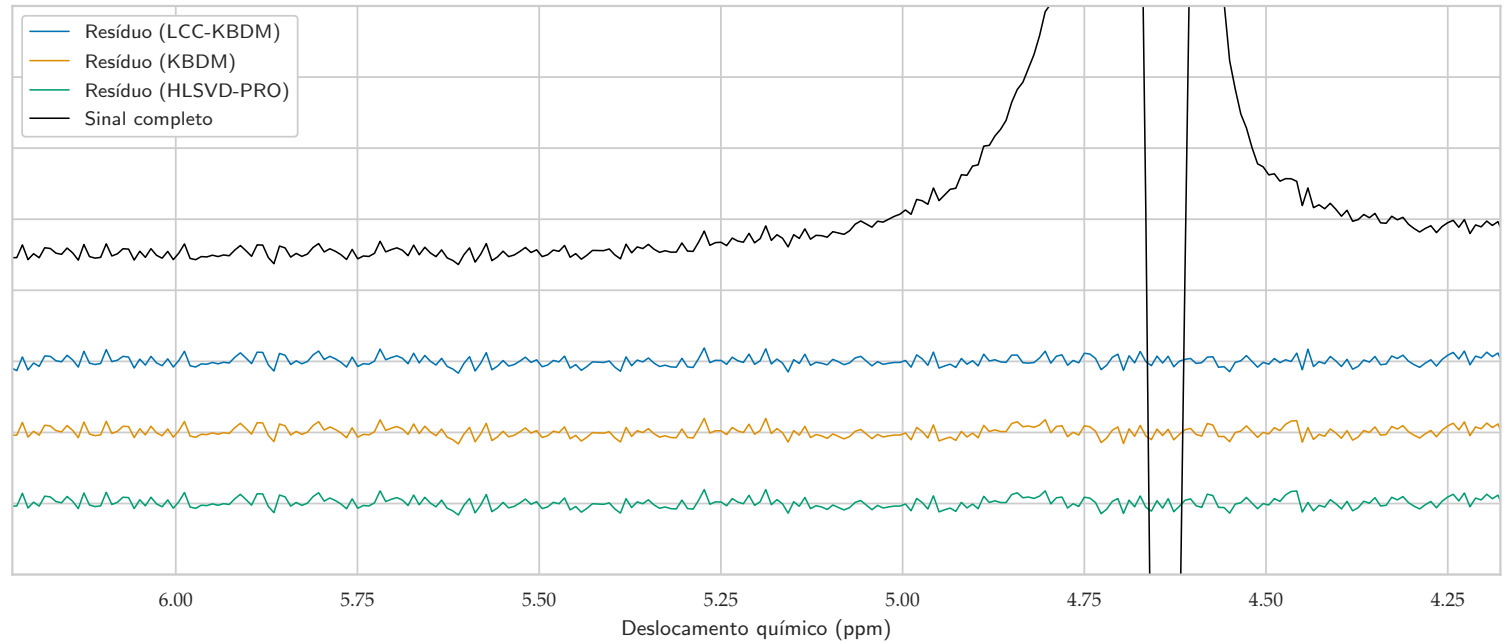

C) Estimativa da distribuição do resíduo na região suprimida
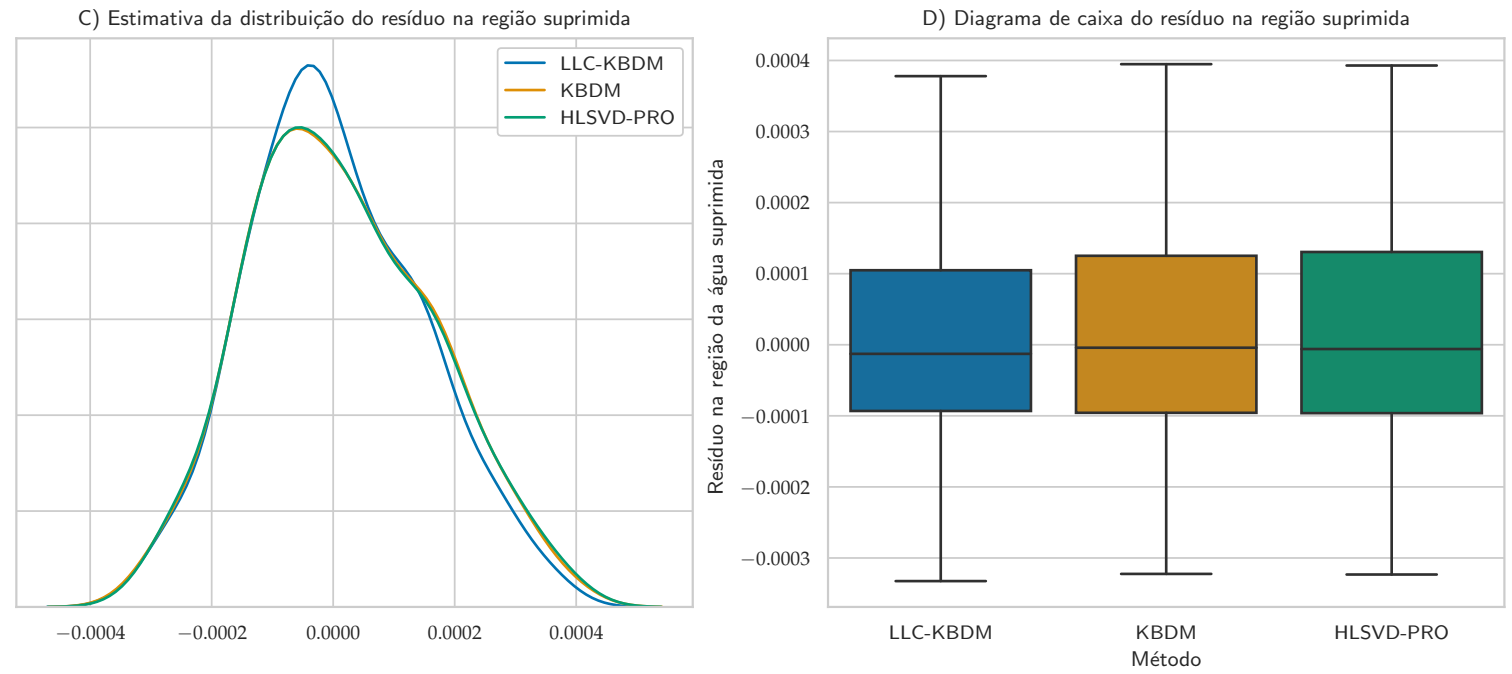

Figura 5.17 - Resultados obtidos para o espectro \#6. As linhas verticais tracejadas em A) denotam a região onde a água foi suprimida. Os pontos pretos nos diagramas de caixa em D) indicam a presença de outliers, caracterizado pela presença de sinal remanescente na região após o procedimento. O sinal completo denota o espectro total, antes da remoção da água.

Fonte: Elaborada pelo autor. 
A) Espectros obtidos após supressão do sinal residual da água

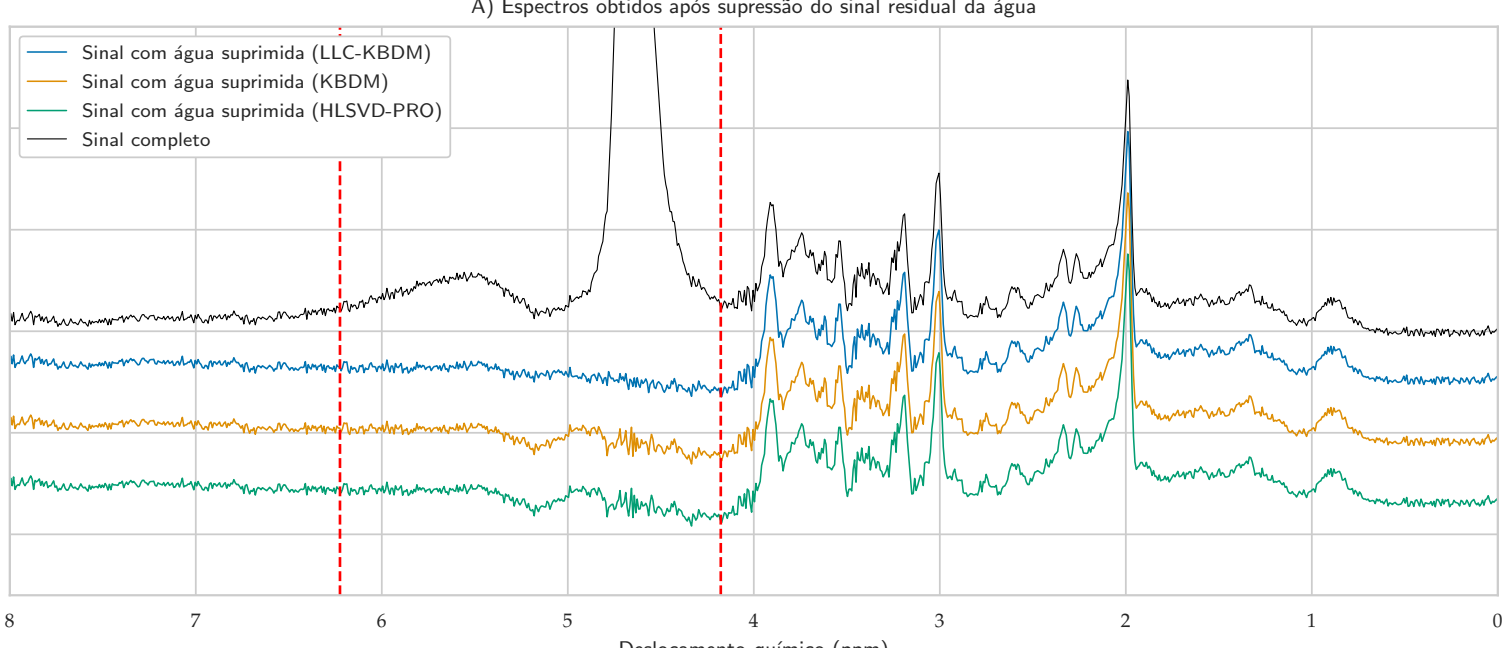

B) Resíduo do sinal estimado na região suprimida

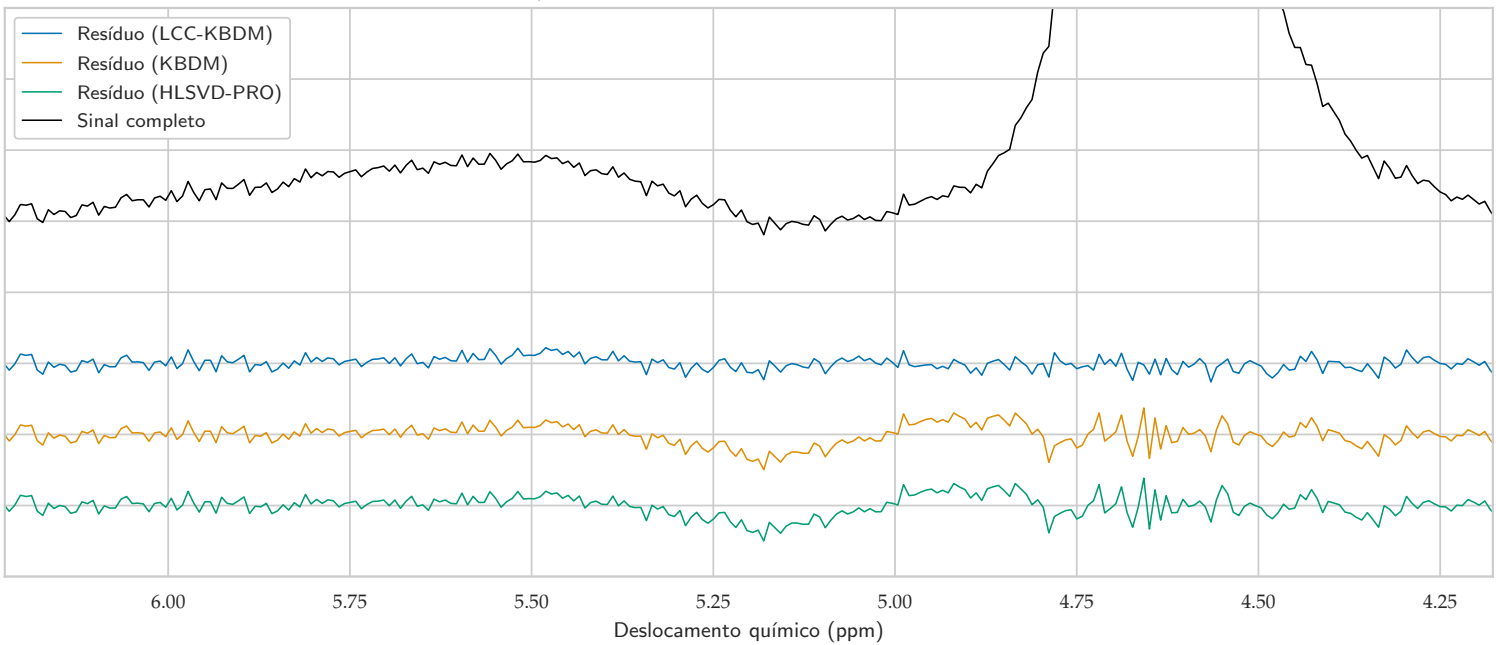

C) Estimativa da distribuição do resíduo na região suprimida
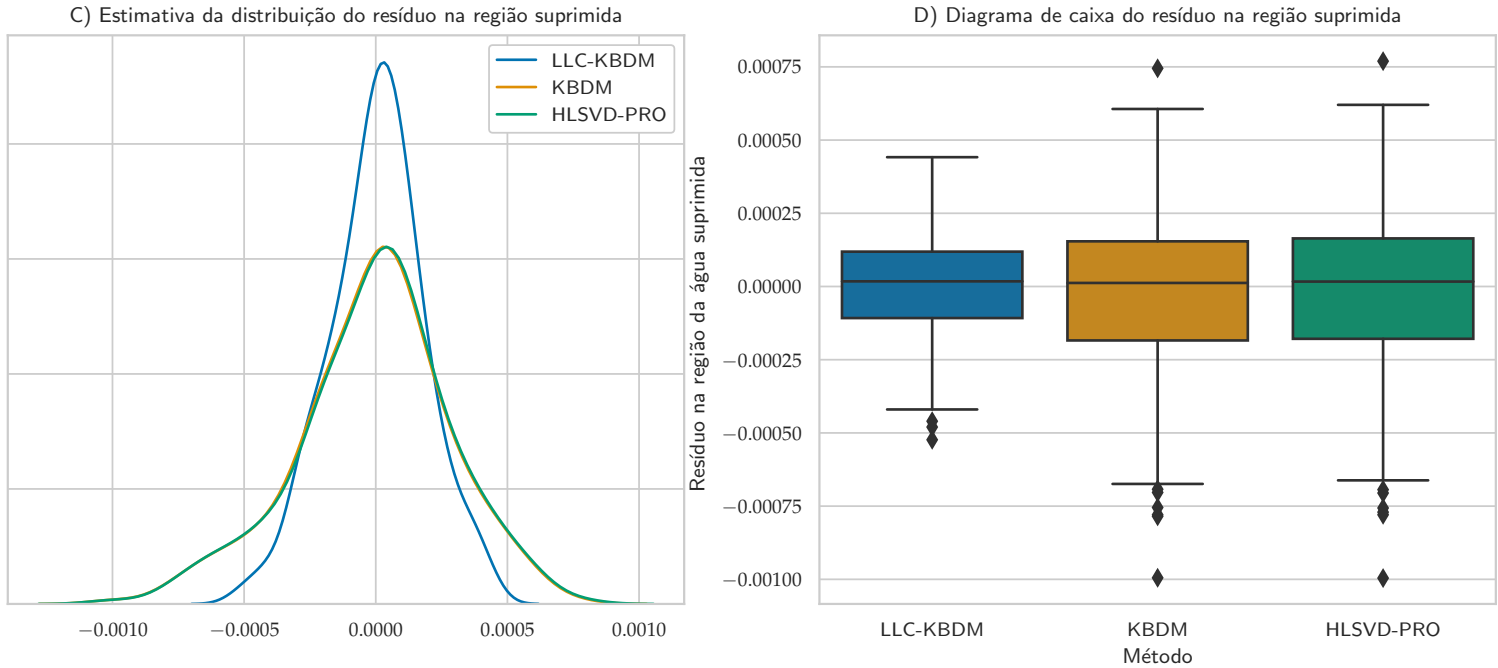

Figura 5.18 - Resultados obtidos para o espectro \#7. As linhas verticais tracejadas em A) denotam a região onde a água foi suprimida. Os pontos pretos nos diagramas de caixa em D) indicam a presença de outliers, caracterizado pela presença de sinal remanescente na região após o procedimento. O sinal completo denota o espectro total, antes da remoção da água.

Fonte: Elaborada pelo autor. 
A) Espectros obtidos após supressão do sinal residual da água

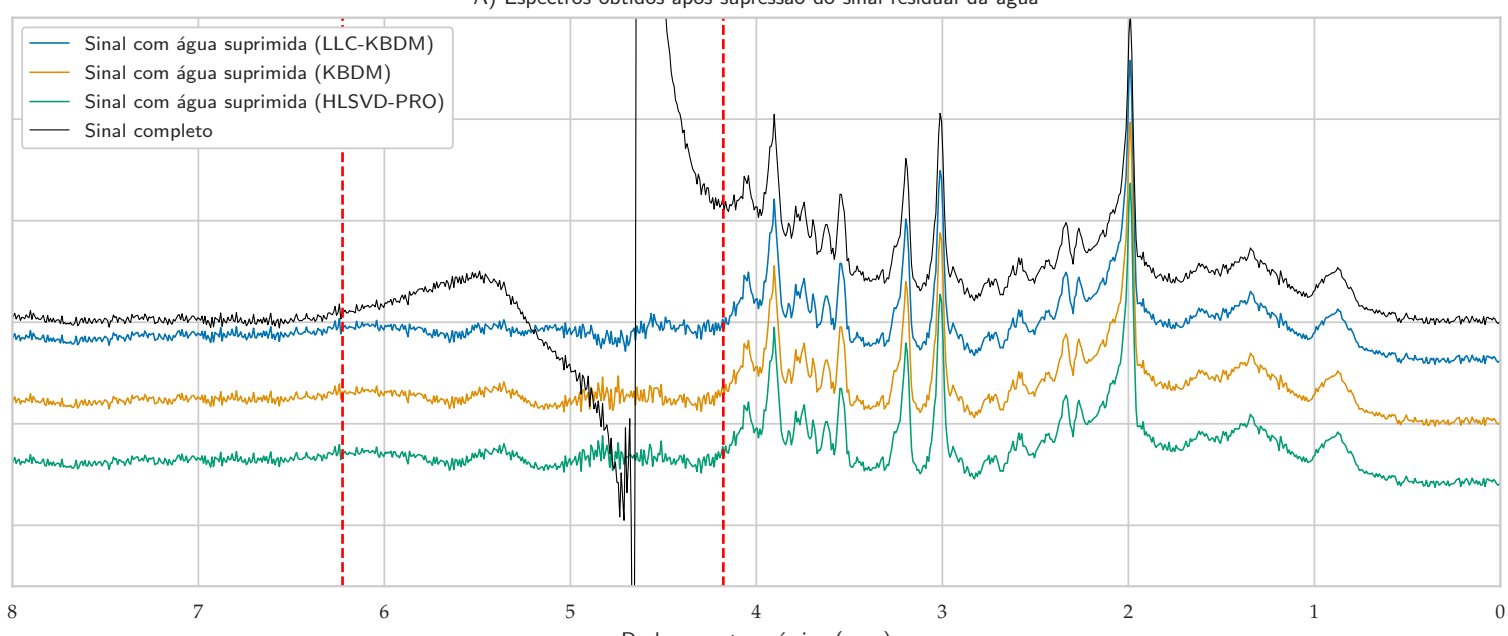

B) Resíduo do sinal estimado na região suprimida

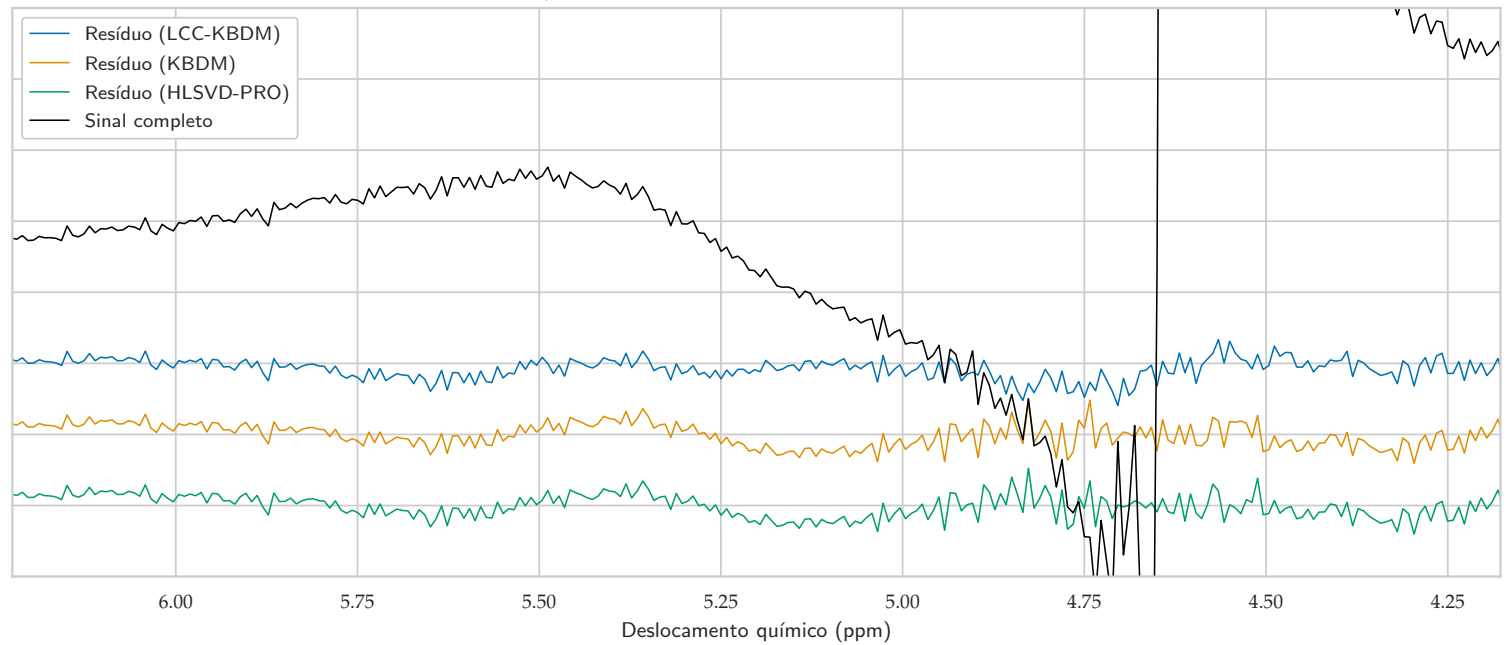

C) Estimativa da distribuição do resíduo na região suprimida
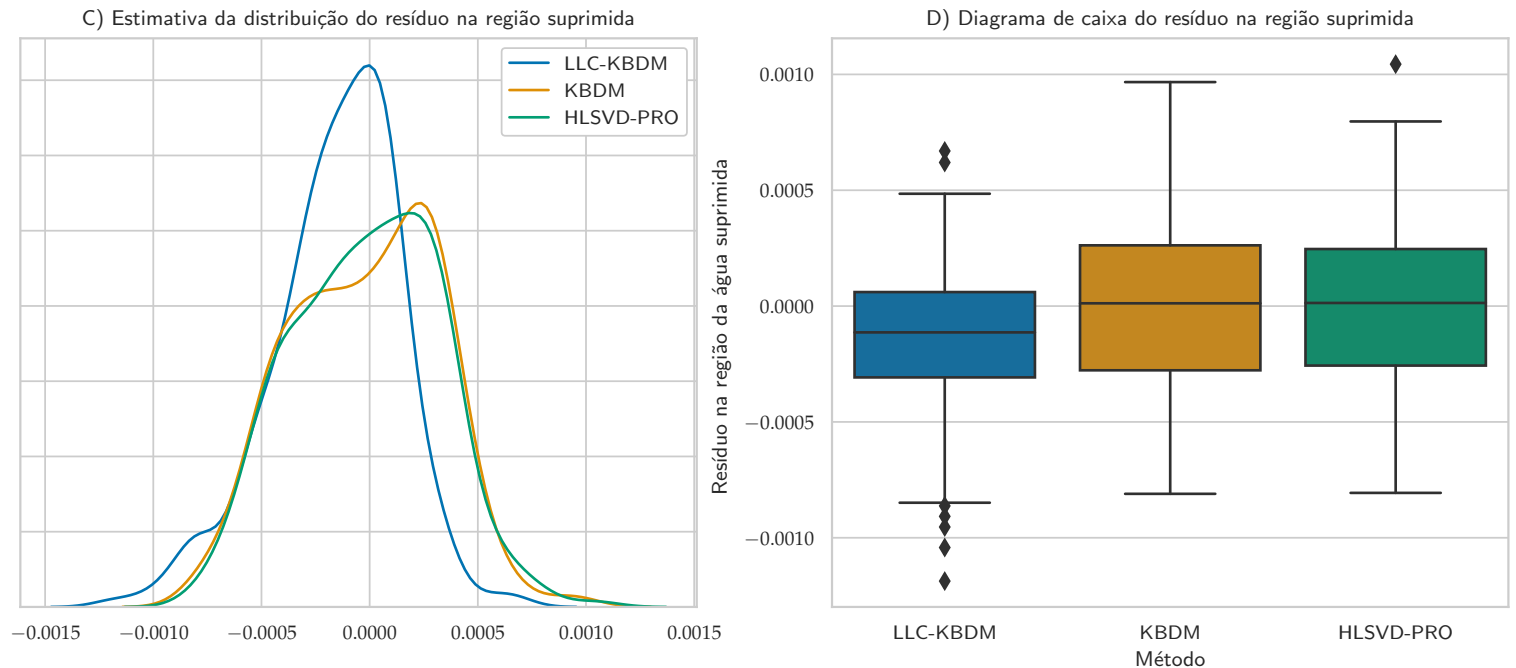

Figura 5.19 - Resultados obtidos para o espectro \#8. As linhas verticais tracejadas em A) denotam a região onde a água foi suprimida. Os pontos pretos nos diagramas de caixa em D) indicam a presença de outliers, caracterizado pela presença de sinal remanescente na região após o procedimento. O sinal completo denota o espectro total, antes da remoção da água.

Fonte: Elaborada pelo autor. 
A) Espectros obtidos após supressão do sinal residual da água

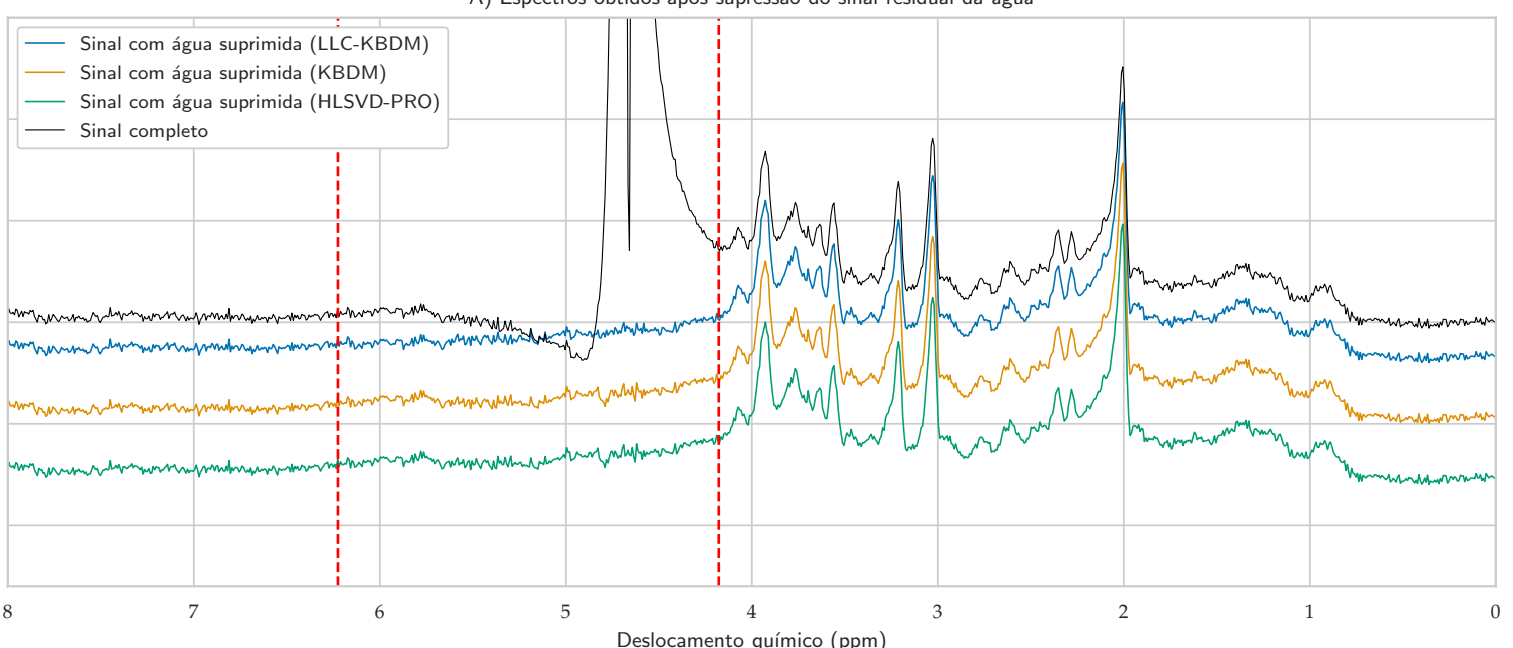

B) Resíduo do sinal estimado na região suprimida

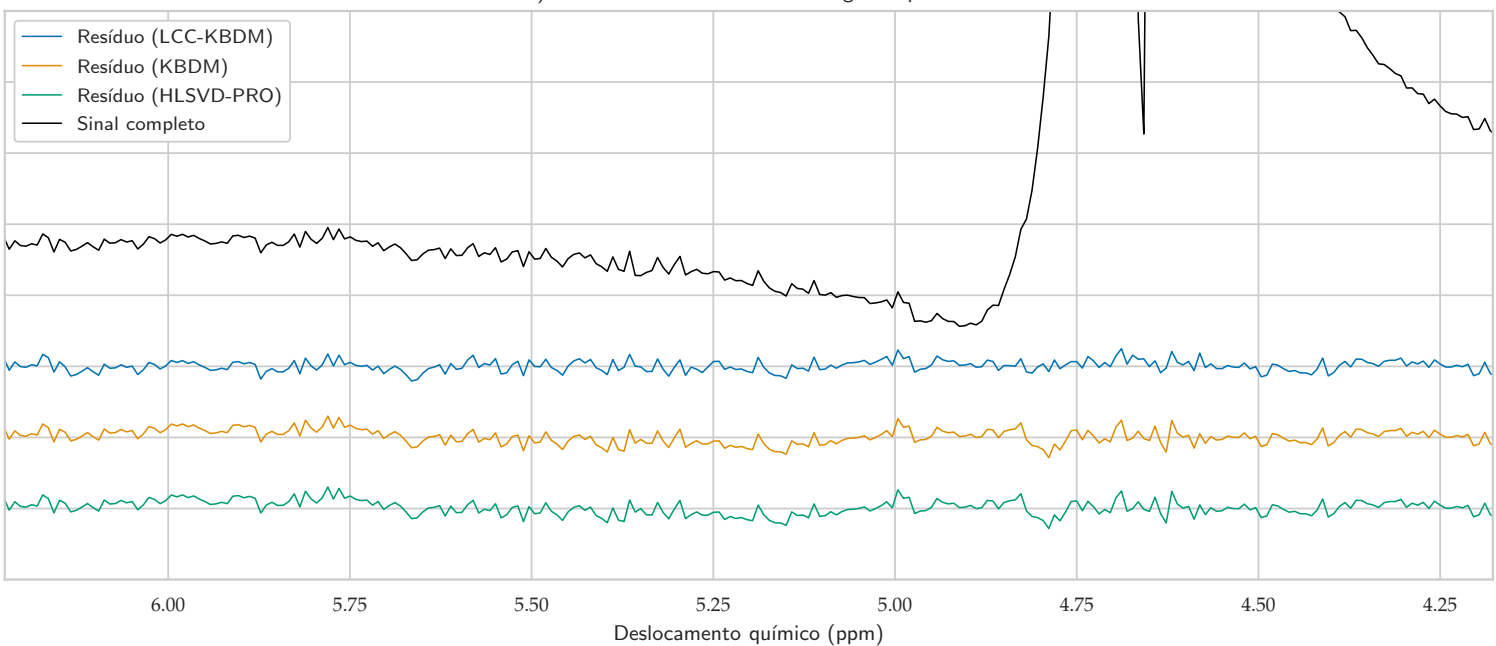

C) Estimativa da distribuição do resíduo na região suprimida
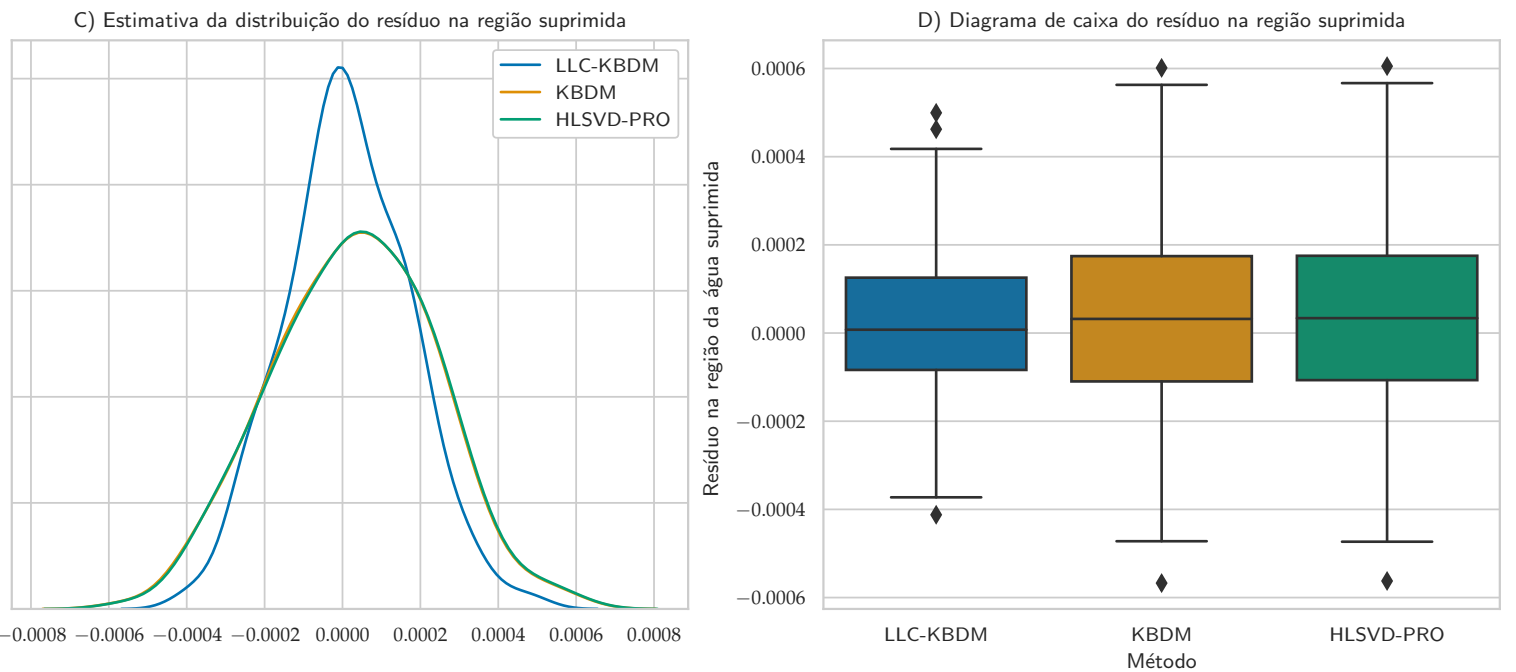

Figura 5.20 - Resultados obtidos para o espectro \#9. As linhas verticais tracejadas em A) denotam a região onde a água foi suprimida. Os pontos pretos nos diagramas de caixa em D) indicam a presença de outliers, caracterizado pela presença de sinal remanescente na região após o procedimento. O sinal completo denota o espectro total, antes da remoção da água.

Fonte: Elaborada pelo autor. 
A) Espectros obtidos após supressão do sinal residual da água

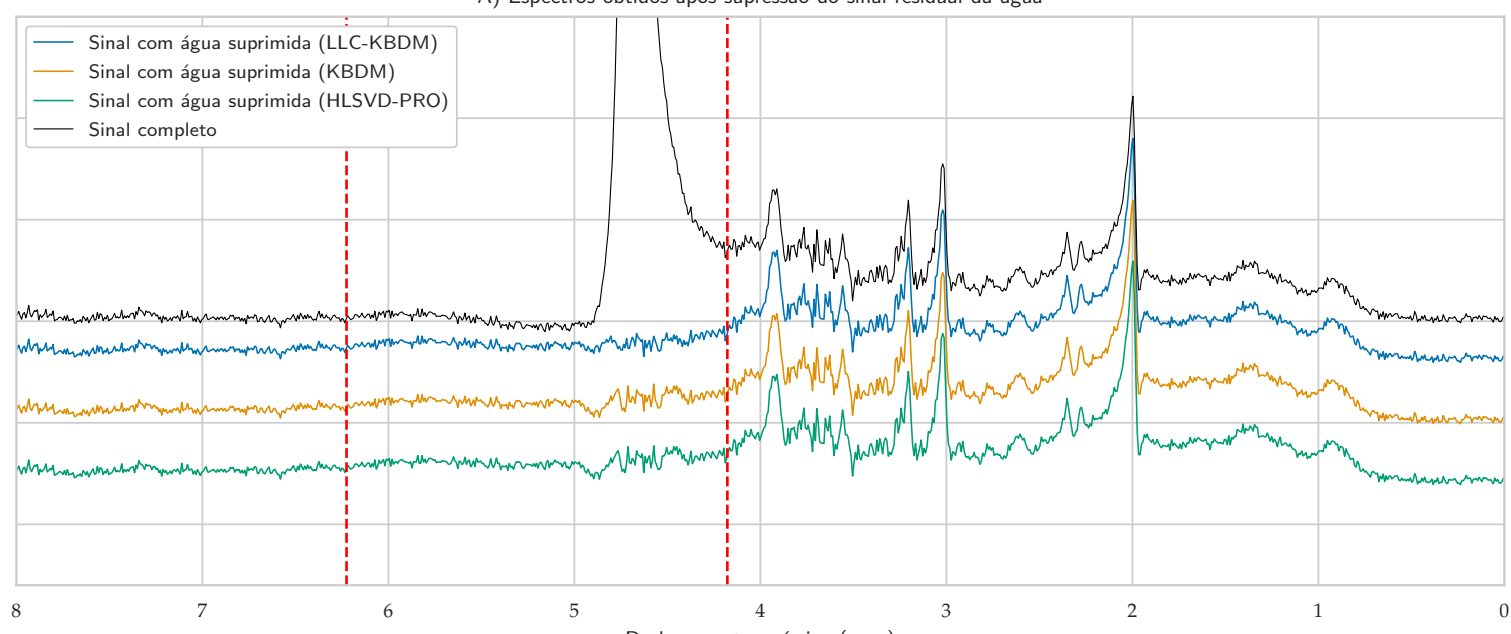

B) Resíduo do sinal estimado na região suprimida

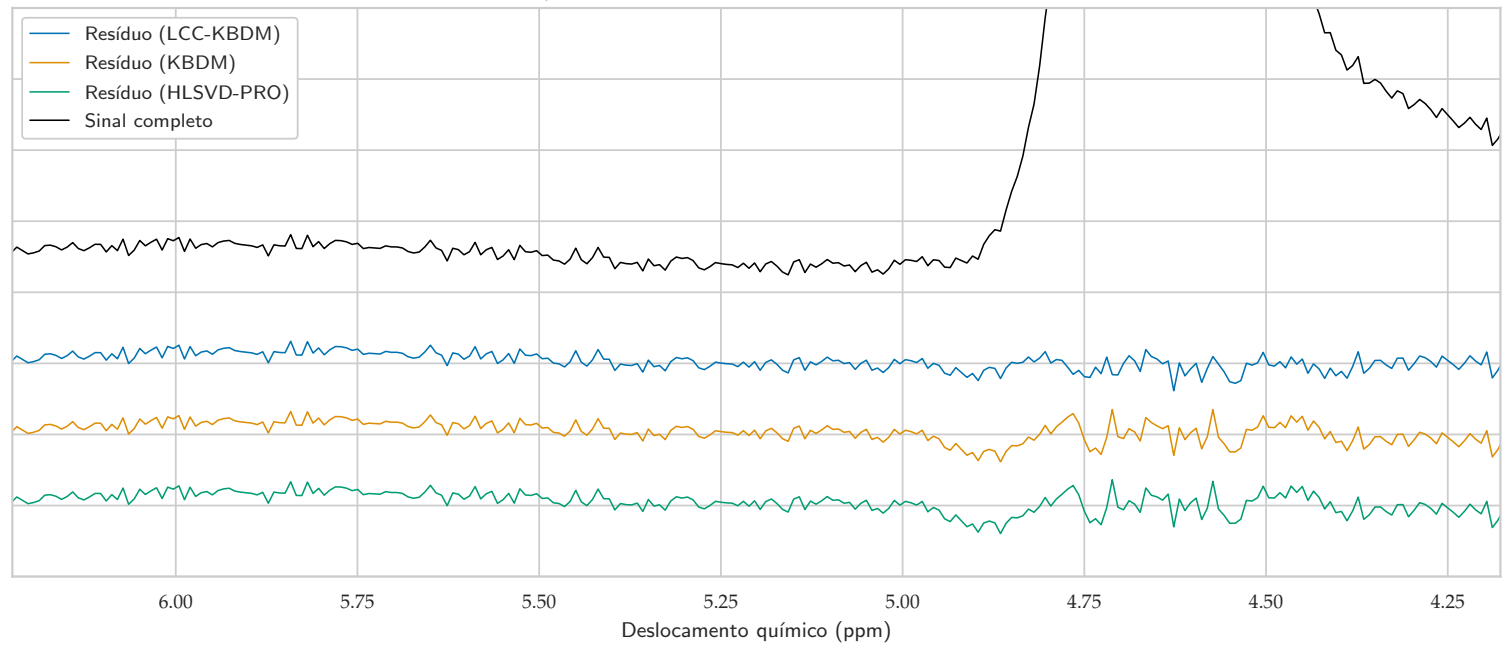

C) Estimativa da distribuição do resíduo na região suprimida
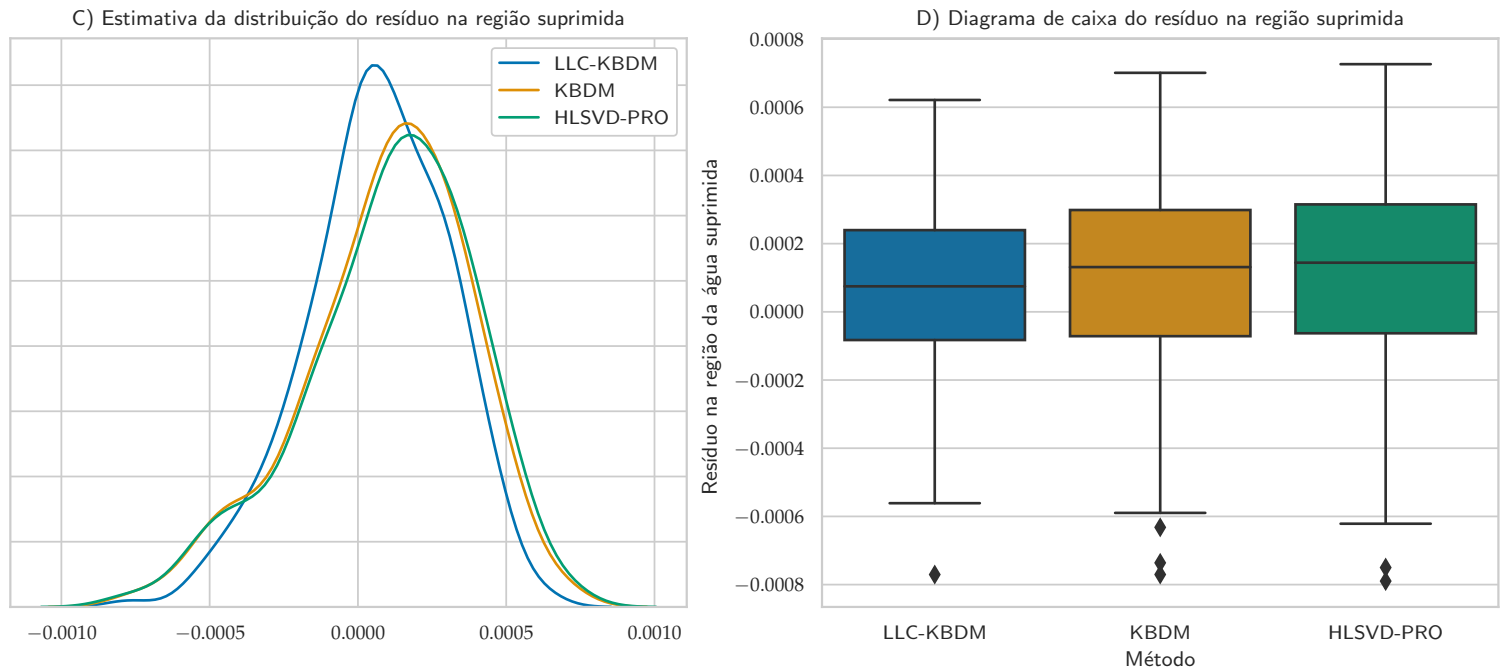

Figura 5.21 - Resultados obtidos para o espectro \#10. As linhas verticais tracejadas em A) denotam a região onde a água foi suprimida. Os pontos pretos nos diagramas de caixa em D) indicam a presença de outliers, caracterizado pela presença de sinal remanescente na região após o procedimento. O sinal completo denota o espectro total, antes da remoção da água.

Fonte: Elaborada pelo autor. 
A) Espectros obtidos após supressão do sinal residual da água

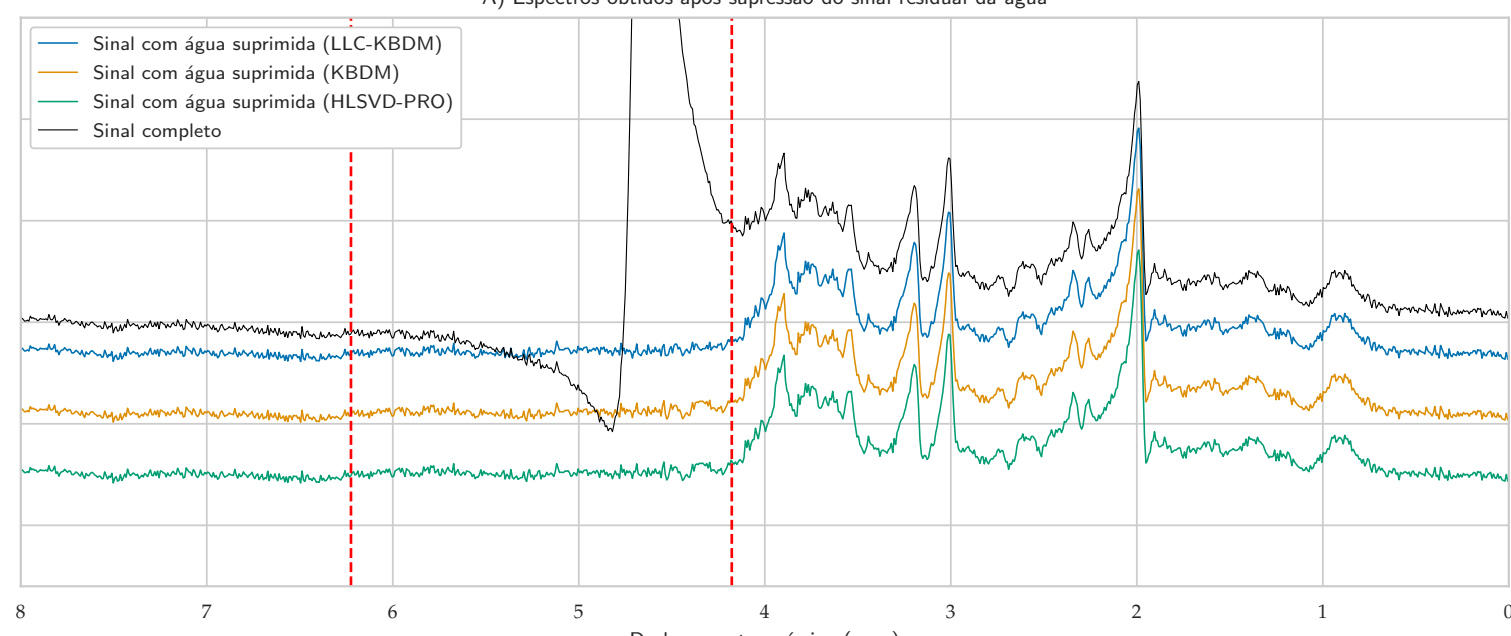

B) Resíduo do sinal estimado na região suprimida

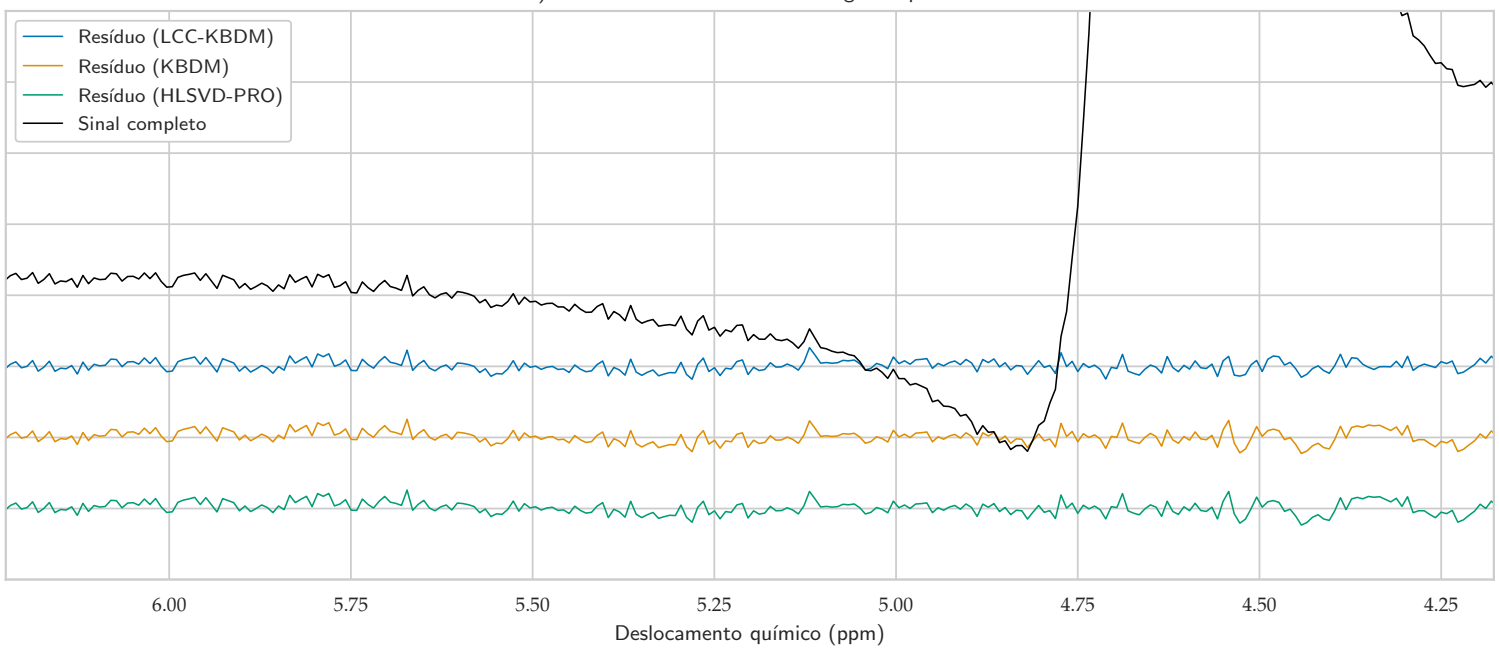

C) Estimativa da distribuição do resíduo na região suprimida
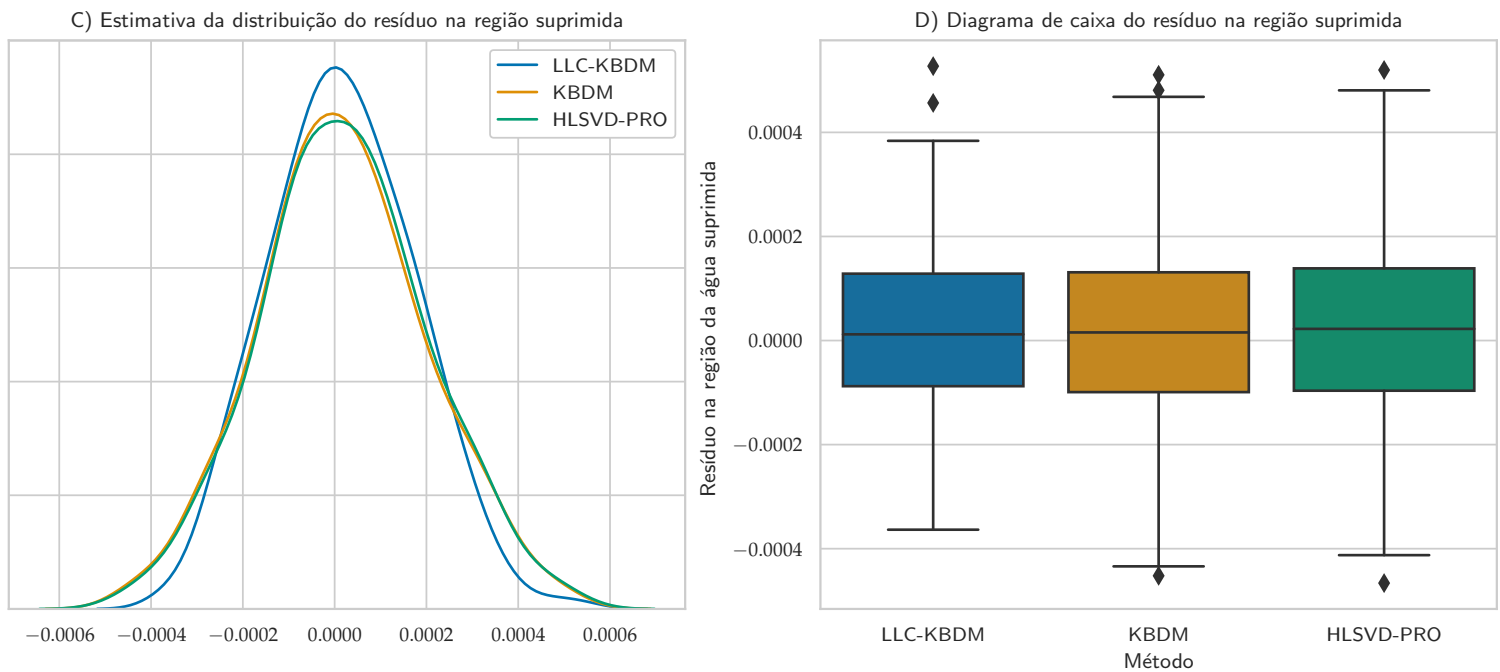

Figura 5.22 - Resultados obtidos para o espectro \#11. As linhas verticais tracejadas em A) denotam a região onde a água foi suprimida. Os pontos pretos nos diagramas de caixa em D) indicam a presença de outliers, caracterizado pela presença de sinal remanescente na região após o procedimento. O sinal completo denota o espectro total, antes da remoção da água.

Fonte: Elaborada pelo autor. 
A) Espectros obtidos após supressão do sinal residual da água

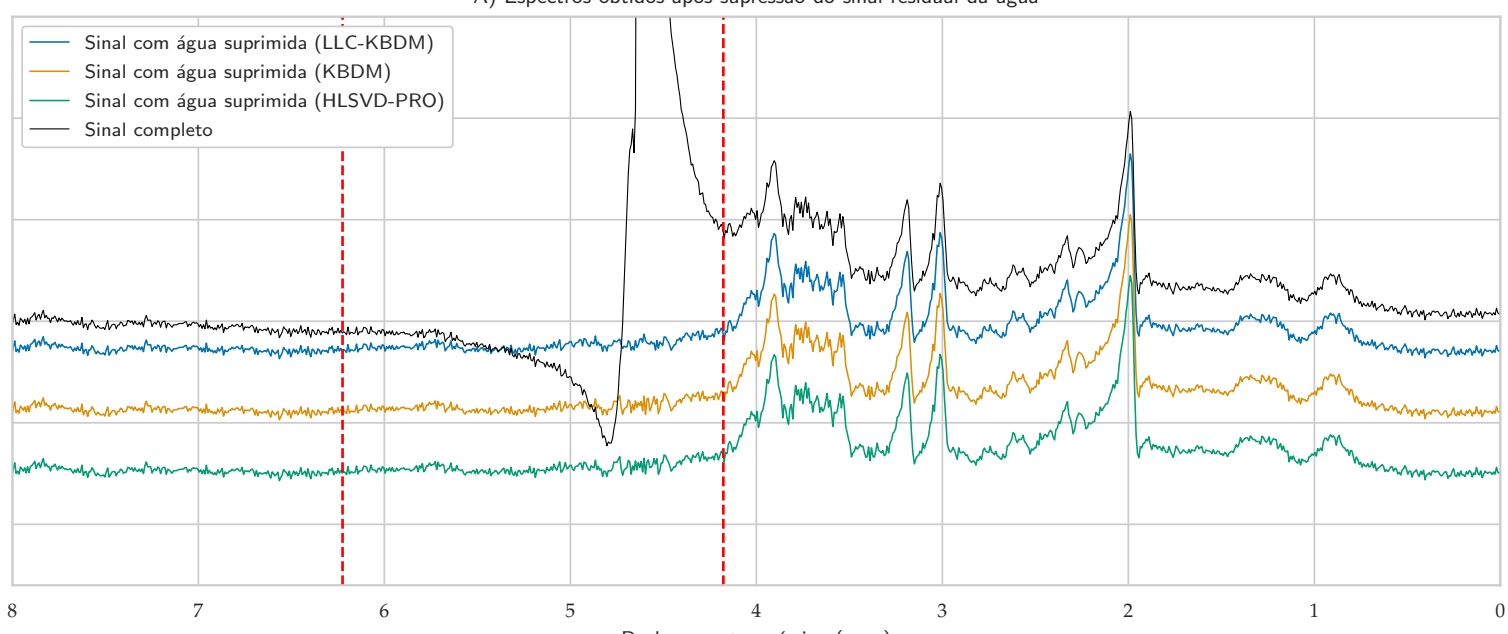

B) Resíduo do sinal estimado na região suprimida

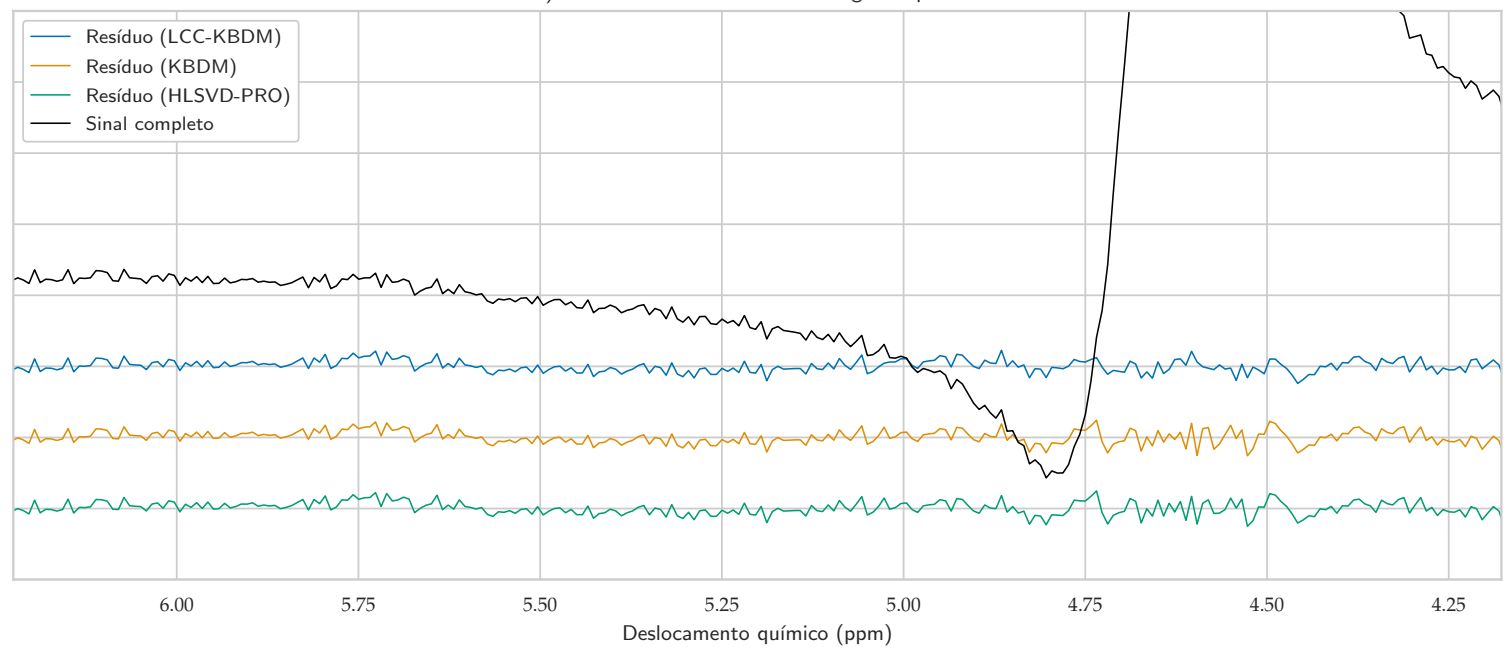

C) Estimativa da distribuição do resíduo na região suprimida
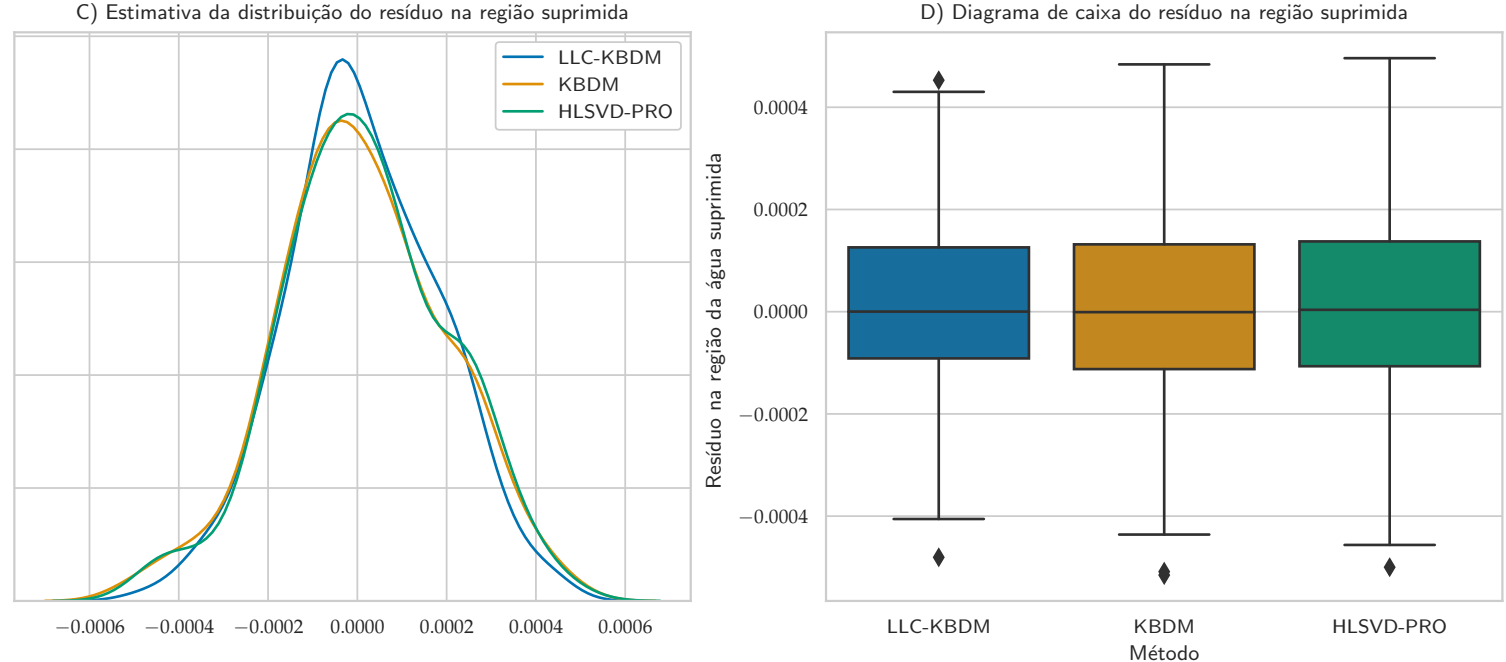

Figura 5.23 - Resultados obtidos para o espectro \#12. As linhas verticais tracejadas em A) denotam a região onde a água foi suprimida. Os pontos pretos nos diagramas de caixa em D) indicam a presença de outliers, caracterizado pela presença de sinal remanescente na região após o procedimento. O sinal completo denota o espectro total, antes da remoção da água.

Fonte: Elaborada pelo autor. 
A) Espectros obtidos após supressão do sinal residual da água

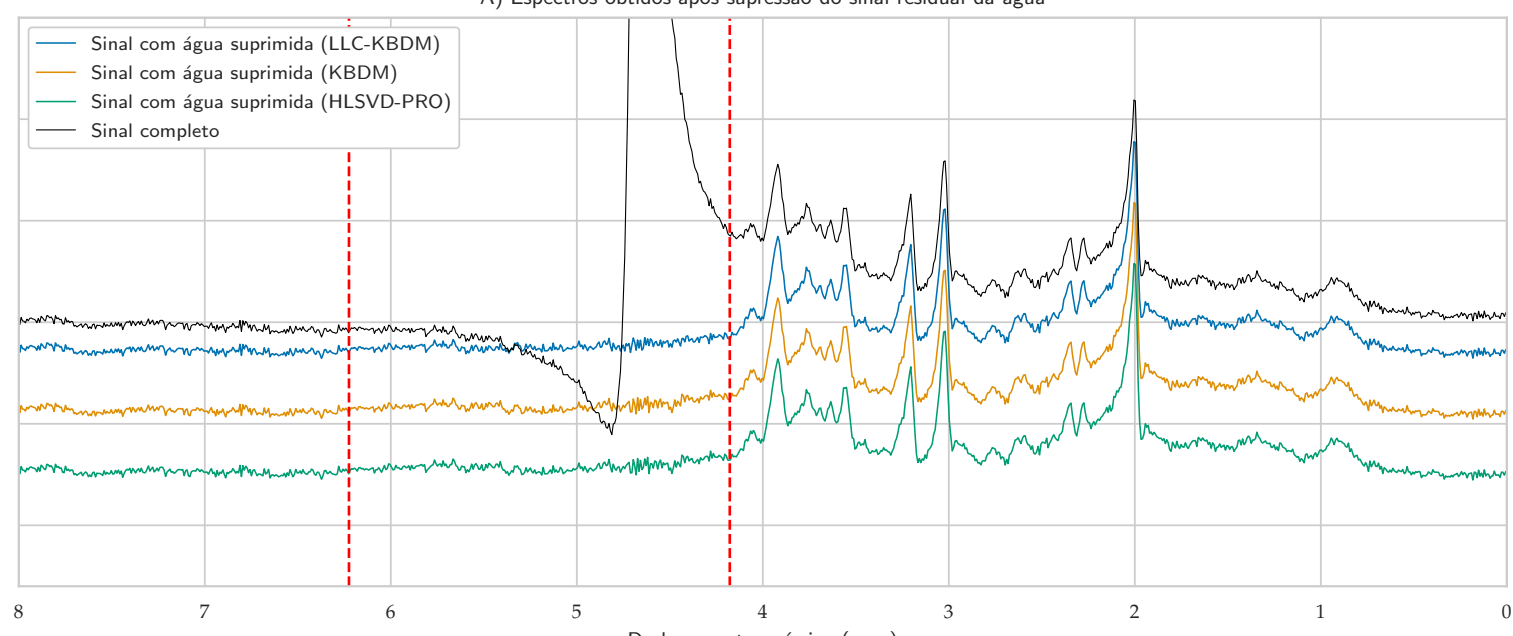

B) Resíduo do sinal estimado na região suprimida

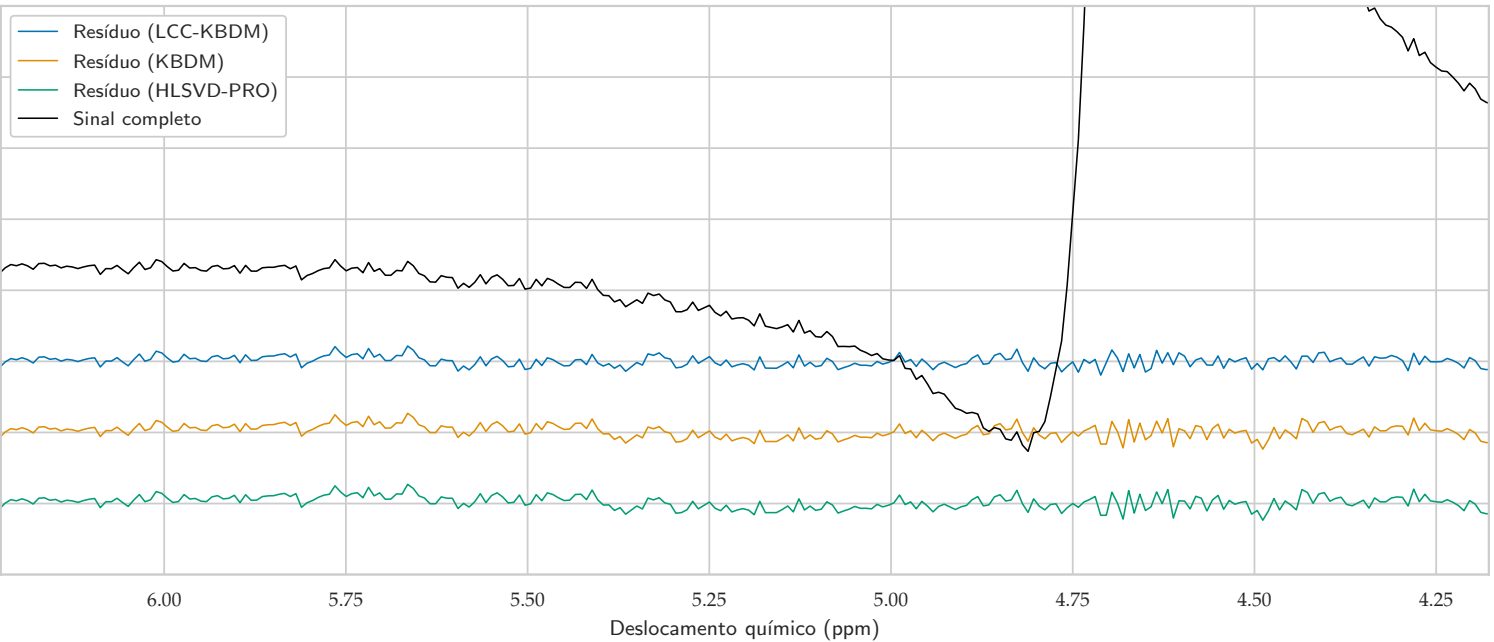

C) Estimativa da distribuição do resíduo na região suprimida
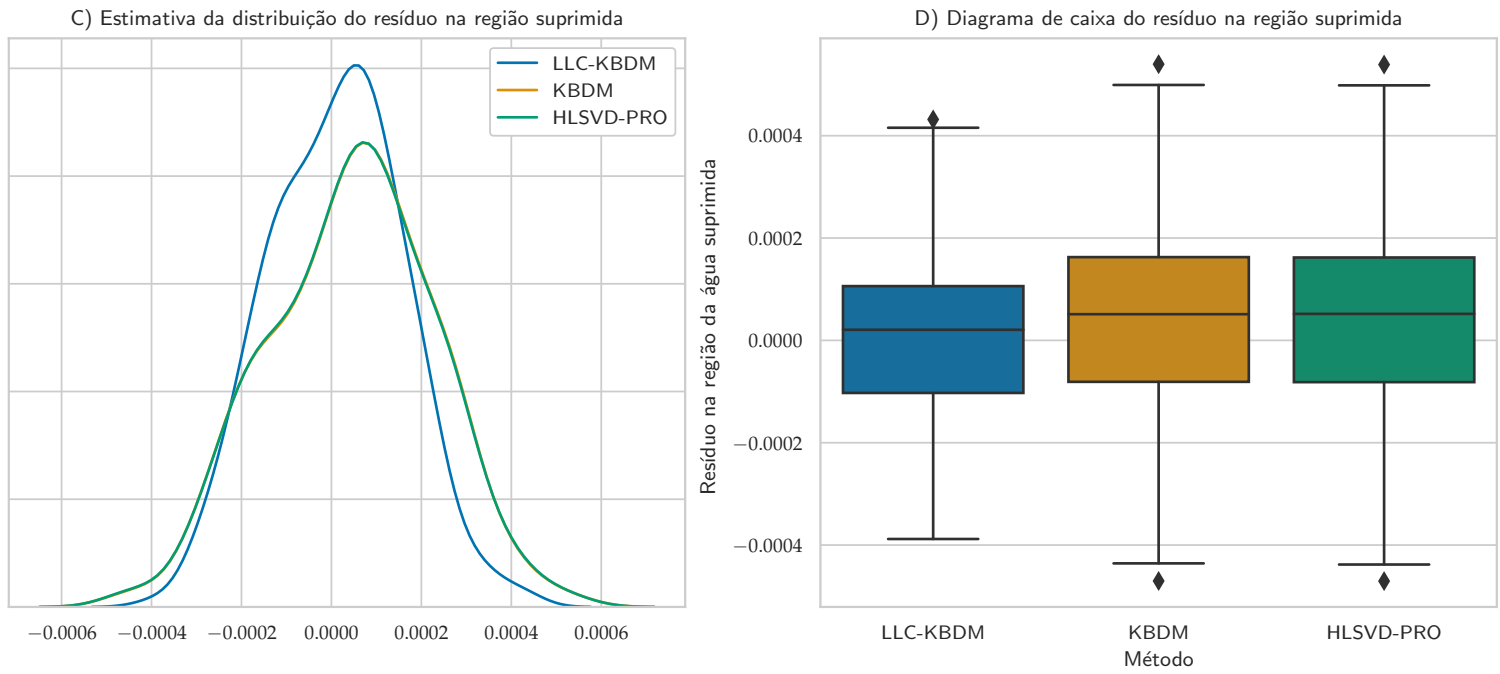

Figura 5.24 - Resultados obtidos para o espectro \#13. As linhas verticais tracejadas em A) denotam a região onde a água foi suprimida. Os pontos pretos nos diagramas de caixa em D) indicam a presença de outliers, caracterizado pela presença de sinal remanescente na região após o procedimento. O sinal completo denota o espectro total, antes da remoção da água.

Fonte: Elaborada pelo autor. 
A) Espectros obtidos após supressão do sinal residual da água

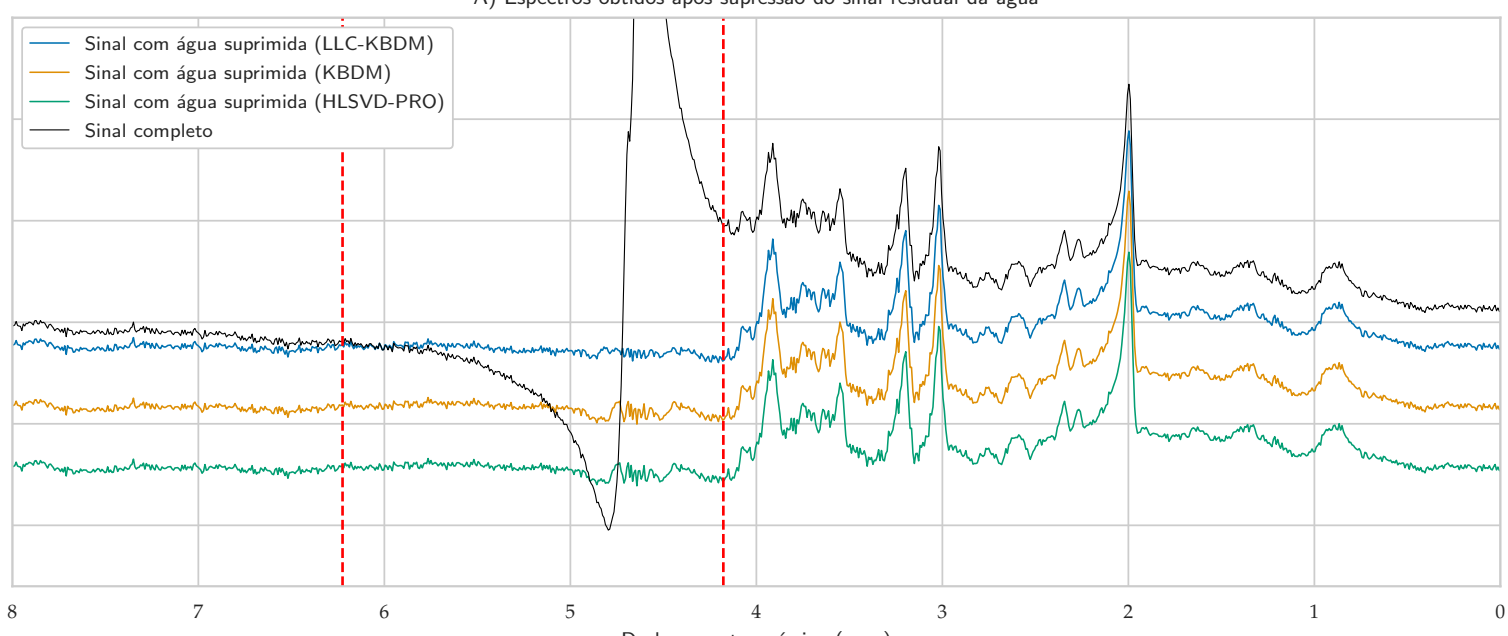

B) Resíduo do sinal estimado na região suprimida

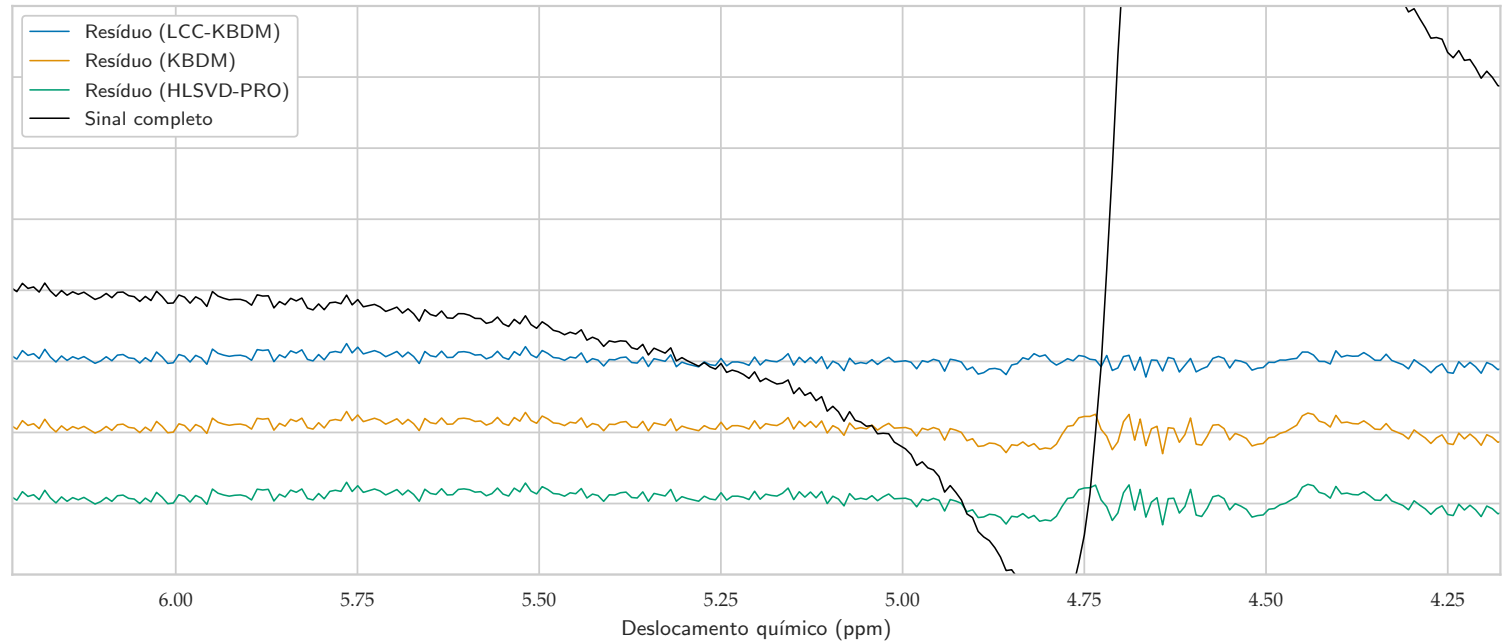

C) Estimativa da distribuição do resíduo na região suprimida

D) Diagrama de caixa do resíduo na região suprimida
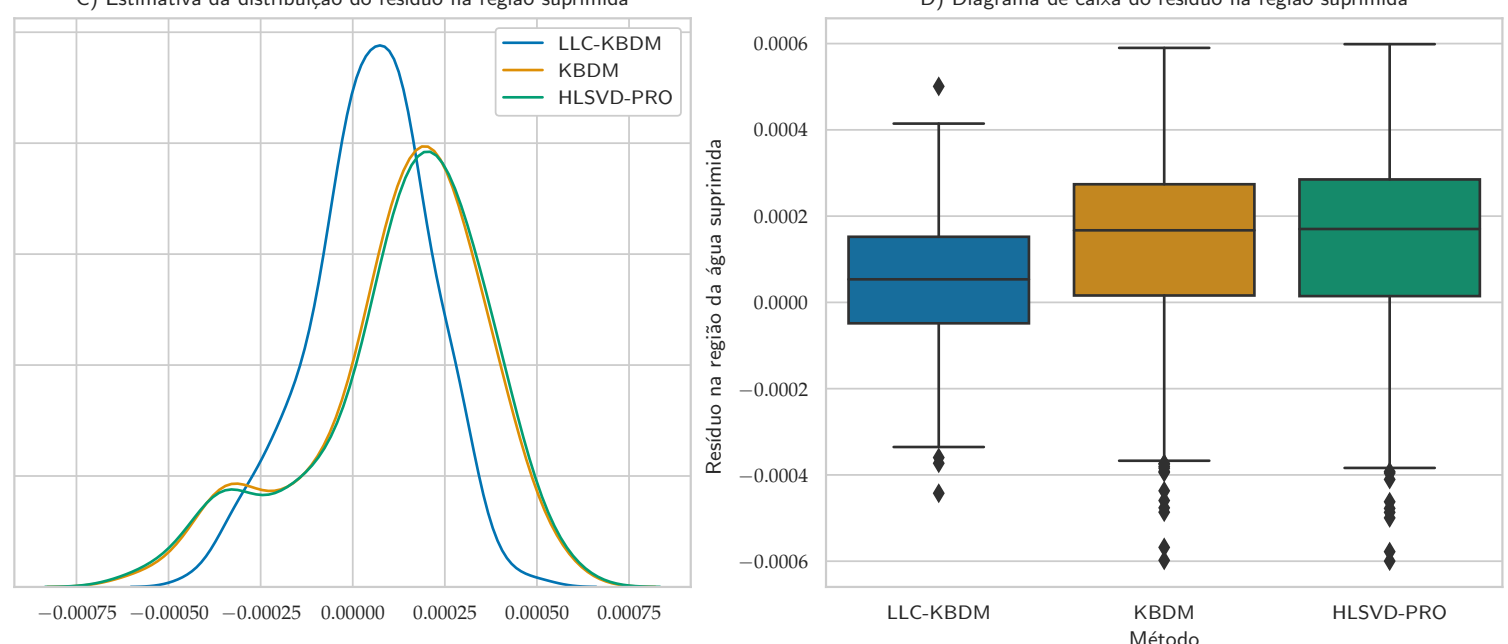

Figura 5.25 - Resultados obtidos para o espectro \#14. As linhas verticais tracejadas em A) denotam a região onde a água foi suprimida. Os pontos pretos nos diagramas de caixa em D) indicam a presença de outliers, caracterizado pela presença de sinal remanescente na região após o procedimento. O sinal completo denota o espectro total, antes da remoção da água.

Fonte: Elaborada pelo autor. 


\section{Conclusão e trabalhos futuros}

O estudo paramétrico para a implementação do KBDM utilizando decomposição em valores singulares na solução do problema de autovalores generalizados foi realizado.

A técnica para a promediação através de agrupamento de linhas espectrais no espaço de parâmetros utilizando múltiplas soluções do KBDM, denominada LLC-KBDM, foi formulada e uma implementação através do HDBSCAN utilizando uma estratégia iterativa foi proposta.

Foram desenvolvidas uma biblioteca de software e uma interface gráfica, MRSuppressor, para o KBDM e o LLC-KBDM.

Comparações entre o KBDM, o LLC-KDBM e o HLSVD-PRO utilizando sinais simulados e sinais in vivo foram feitas. Os resultados mostram que o KBDM é ligeiramente superior ao HLSVD-PRO em todos os níveis de ruído considerados. Por outro lado, o LLC-KBDM se mostrou mais adequado para sinais os mais ruidosos, embora algumas instabilidades numéricas tenham sido observadas no caso contendo o maior nível de ruído. Para sinais in vivo, a tarefa da remoção do sinal residual da água é comparada entre os mesmos métodos. De acordo com o teste estatístico utilizado para o nível de significância $\alpha=0.05$, o sinal residual da água para 10 dos 14 espectros foram adequadamente suprimidos pelo LLC-KBDM; enquanto o KBDM e HLSVD-PRO conseguiram o mesmo feito para apenas 6 e 7 espectros, respectivamente.

Diversos aspectos das técnicas estudadas ainda podem ser aprimorados. O HLSVD-PRO utiliza uma técnica de decomposição parcial em valores singulares (SVD) que explora a esparsidade do problema e a estrutura da matriz de Hankel envolvida. Esta estratégia impulsionou o desempenho dos algoritmos baseados no formalismo do espaço de estados. A decomposição SVD da LAPACK foi utilizada na implementação do KBDM e, portanto, é desconhecido o ganho que o KBDM e, consequentemente, o LLC-KBDM poderiam ter ao fazer uso da mesma estratégia utilizada pelo HLSVD-PRO.

Uma limitação do LLC-KBDM consiste no seu custo computacional. No entanto, o algoritmo é altamente paralelizável durante o processo de amostragem das soluções do KBDM, que podem ser calculadas de maneira independente. Versões paralelas de alto desempenho para o LLC-KBDM ainda podem ser desenvolvidas. 
O uso de conhecimento a priori pode ser explorado durante o agrupamento das linhas espectrais. Por exemplo, picos de referência podem ser utilizados na busca de linhas que tenham similaridade com as múltiplas estimativas do KBDM.

A técnica da promediação no espaço de parâmetros pode ser adaptada em outros métodos, como no próprio HLSVD-PRO ou ainda utilizando soluções híbridas provenientes de outros algoritmos. 


\section{REFERÊNCIAS}

1 BÉDARD, M.-J.; CHANTAL, S. Brain magnetic resonance spectroscopy in obsessive-compulsive disorder: the importance of considering subclinical symptoms of anxiety and depression. Psychiatry Research: neuroimaging, v. 192, n. 1, p. 45-54, 2011 . doi: 10.1016/j.pscychresns.2010.10.008.

2 YÜCEL, M.; WOOD, S. J.; WELLARD, R. M.; HARRISON, B. J.; FORNITO, A.; PUJOL, J.; VELAKOULIS, D.; PANTELIS, C. Anterior cingulate glutamate-glutamine levels predict symptom severity in women with obsessive-compulsive disorder. Australian and New Zealand Journal of Psychiatry, v. 42, n. 6, p. 467-477, 2008. doi: 10.1080/00048670802050546.

3 TIBBO, P.; HANSTOCK, C.; VALIAKALAYIL, A.; ALLEN, P. 3-T proton MRS investigation of glutamate and glutamine in adolescents at high genetic risk for schizophrenia. The American Journal of Psychiatry, v. 161, n. 6, p. 1116-8, 2004. doi: 10.1176/appi.ajp.161.6.1116.

4 KANTARCI, K.; REYNOLDS, G.; PETERSEN, R. C.; BOEVE, B. F.; KNOPMAN, D. S.; EDLAND, S. D.; SMITH, G. E.; IVNIK, R. J.; TANGALOS, E. G.; JACK, C. R. Proton MR spectroscopy in mild cognitive impairment and Alzheimer disease: comparison of 1.5 and $3 \mathrm{~T}$. American Journal of Neuroradiology, v. 24, n. 5, p. 843-849, 2003.

$5 \mathrm{KANTARCI}, \mathrm{K} .1 \mathrm{H}$ magnetic resonance spectroscopy in dementia. The British Journal of Radiology, v. 80, p. S146-52, 2007. doi: 10.1259/bjr/60346217.

6 BRIGHAM, E. O. The fast Fourier transform and its applications. Englewood Cliff: PrenticeHall, 1988.

7 CALLAGHAN, P. T. Principles of nuclear magnetic resonance microscopy. Clarendon Press, 1993.

8 GERLACH, W.; STERN, O. Das magnetische moment des silberatoms. Zeitschrift für Physik, v. 9, n. 1, p. 353-355, 1922. doi: 10.1007/BF01326984.

9 LANDÉ, A. Nuclear magnetic moments and their origin. Physical Review, v. 46, n. 6, p. 477-480, 1934. doi: 10.1103/PhysRev.46.477.

10 BLOCH, F. Nuclear induction. Physical Review, v. 70, p. 460-474, 1946 . doi: 10.1103/PhysRev.70.460.

11 HELMS, G. The principles of quantification applied to in vivo proton MR spectroscopy. European Journal of Radiology, v. 67, n. 2, p. 218-229, 2008. doi: 10.1016/j.ejrad.2008.02.034. 
12 HAHN, E. L.; MAXWELL, D. E. Spin echo measurements of nuclear spin coupling in molecules. Physical Review, v. 88, p. 1070-1084, 1952. doi: 10.1103/PhysRev.88.1070.

13 NEAR, J. Spectral quantification and pitfalls in interpreting magnetic resonance spectroscopic data: what to look out for. In: STAGG, C.; ROTHMAN, D. (Ed.). Magnetic resonance spectroscopy: tools for neuroscience research and emerging clinical application. Berlin: Elsevier, 2014.

14 JANSEN, J. F. A.; BACKES, W. H.; KLAAS, N.; KOOI, M. E. $1 \mathrm{H}$ MR spectroscopy of the brain: absolute quantification of metabolites. Radiology, v. 240, n. 2, p. 318-332, 2006. doi: $10.1148 /$ radiol.2402050314.

15 PROVENCHER, S. W. Estimation of metabolite concentrations from localizedin vivo proton NMR spectra. Magnetic Resonance in Medicine, v. 30, n. 6, p. 672-679, 1993. doi: 10.1002/mrm.1910300604.

16 KUNG, S. Y.; ARUN, K. S.; Bhaskar Rao, D. V. State-space and singular-value decomposition-based approximation methods for the harmonic retrieval problem. Journal of the Optical Society of America, v. 73, n. 12, p. 1799, 1983. doi: 10.1364/JOSA.73.001799.

17 BARKHUIJSEN, H.; DE BEER, R.; VAN ORMONDT, D. Improved algorithm for noniterative time-domain model fitting to exponentially damped magnetic resonance signals. Journal of Magnetic Resonance (1969), v. 73, n. 3, p. 553-557, 1987. doi: 10.1016/00222364(87)90023-0.

18 CABANES, E.; CONFORT-GOUNY, S.; FUR, Y. L.; SIMOND, G.; COZZONE, P. Optimization of residual water signal removal by HLSVD on simulated short echo time proton MR spectra of the human brain. Journal of Magnetic Resonance, v. 150, n. 2, p. $116-125,2001$. doi: https://doi.org/10.1006/jmre.2001.2318.

19 VANHAMME, L.; FIERRO, R. D.; Van Huffel, S.; DE BEER, R. Fast removal of residual water in proton spectra. Journal of Magnetic Resonance, v. 132, n. 2, p. 197-203, 1998. doi: 10.1006/jmre.1998.1425.

20 MANDELSHTAM, V. A.; TAYLOR, H. S. Harmonic inversion of time signals and its applications. Journal of Chemical Physics, v. 107, n. 17, p. 6756, 1997. doi: 10.1063/1.475324.

21 MANDELSHTAM, V. A. FDM: the filter diagonalization method for data processing in NMR experiments. Progress in Nuclear Magnetic Resonance Spectroscopy, v. 38, n. 2, p. 159-196, 2001. doi: 10.1016/S0079-6565(00)00032-7.

22 WALL, M. R.; NEUHAUSER, D. Extraction, through filter-diagonalization, of general quantum eigenvalues or classical normal mode frequencies from a small number of residues or a short-time segment of a signal. I. theory and application to a quantum-dynamics model. Journal of Chemical Physics, v. 102, n. 20, p. 8011-8022, 1995. doi: 10.1063/1.468999. 
23 MAGON, J. A inversão harmônica do espectro de ressonância magnética: uma solução para o problema dos autocampos. 2007. Tese (Livre Docência) - Instituto de Física de São Carlos, Universidade de São Paulo, São Carlos, 2007.

24 MAGON, C.; LIMA, J.; DONOSO, J.; LAVAYEN, V.; BENAVENTE, E.; NAVAS, D.; GONZALEZ, G. Deconvolution of the EPR spectra of vanadium oxide nanotubes. Journal of Magnetic Resonance, v. 222, p. 26 - 33, 2012. doi: https://doi.org/10.1016/j.jmr.2012.06.004.

25 NUNES, L. M.; MORAES, T. B.; BARBOSA, L. L.; MAZO, L. H.; COLNAGO, L. A. Monitoring electrochemical reactions in situ using steady-state free precession 13C NMR spectroscopy. Analytica Chimica Acta, v. 850, p. 1 - 5, 2014 . doi: https://doi.org/10.1016/j.aca.2014.05.022.

26 MORAES, T. O método da diagonalização filtrada (FDM) e suas aplicações para a ressonância magnética. 2011. 100 p. Dissertação (Mestrado em Ciências) - Instituto de Física de São Carlos, Universidade de São Paulo, São Carlos, 2011.

27 MORAES, T. B.; SANTOS, P. M.; MAGON, C. J.; COLNAGO, L. A. Suppression of spectral anomalies in SSFP-NMR signal by the Krylov basis diagonalization method. Journal of Magnetic Resonance, v. 243, p. 74 - 80, 2014 . doi: https://doi.org/10.1016/j.jmr.2014.03.009.

28 SILVA, C. KBDM como ferramenta para processamento de sinais de espectroscopia por ressonância magnética. 2013. 161 p. Dissertação (Mestrado em Ciências) - Instituto de Física de São Carlos, Universidade de São Paulo, São Carlos, 2013.

29 LAUDADIO, T.; MASTRONARDI, N.; VANHAMME, L.; Van Hecke, P.; Van Huffel, S. Improved Lanczos algorithms for blackbox MRS data quantitation. Journal of Magnetic Resonance, v. 157, n. 2, p. 292-297, 2002. doi: 10.1006/jmre.2002.2593.

30 PRONY, R. Essai experimental et analytique sure les lois de la dilatabilité des fluides élastiques et sur celles de la force expansive de la vapeur de l'alkool, á différentes températures. Journal de l'École Politechnique 1, v. 1, Cahier 2, p. 24-76, 1795.

31 RODRÍGUEZ, A. F.; RODRIGO, L. S.; GUILLÉN, E. L.; ASCARIZ, J. M. R.; JIMÉNEZ, J. M. M.; BOQUETE, L. Coding Prony's method in MATLAB and applying it to biomedical signal filtering. BMC Bioinformatics, v. 19, n. 1, p. 451, 2018. doi: 10.1186/s12859-0182473-y.

32 AKKIN, T.; SALIU, S. Estimation of evoked potentials using total least squares Prony technique. Medical \& Biological Engineering \& Computing, v. 36, n. 5, p. 544-548, 1998. doi: $10.1007 /$ BF02524421.

33 VITI, V.; RAGONA, R.; GUIDONI, L.; BARONE, P.; FURMAN, E.; DEGANI, H. Hormonally induced modulation in the phosphate metabolites of breast cancer: analysis of in vivo 
31P MRS signals with a modified Prony method. Magnetic Resonance in Medicine, v. 38, $\mathrm{n}$. 2, p. 285-295, 1997. doi: 10.1002/mrm.1910380219.

34 STEEDLY, W.; YING, C.-H.; MOSES, R. A modified TLS-Prony method using data decimation. IEEE Transactions on Signal Processing, v. 42, n. 9, p. 2292-2303, 1994. doi: $10.1109 / 78.317852$.

35 TUFTS, D.; KUMARESAN, R. Singular value decomposition and improved frequency estimation using linear prediction. IEEE Transactions on Acoustics, Speech, and Signal Processing, v. 30, n. 4, p. 671-675, 1982. doi: 10.1109/TASSP.1982.1163927.

36 BARKHUIJSEN, H.; De Beer, R.; BOVEE, W. M. M. J.; CREYGHTON, J. H. N.; Van Ormondt, D. Application of linear prediction and singular value decomposition (LPSVD) to determine NMR frequencies and intensities from the FID. Magnetic Resonance in Medicine, v. 2, n. 1, p. 86-89, 1985. doi: 10.1002/mrm.1910020111.

37 VANHUfFEL, S.; CHEN, H.; DECANNIERE, C.; VANHECKE, P. Algorithm for timedomain NMR data fitting based on total least squares. Journal of Magnetic Resonance $A, \mathrm{v}$. 110, n. 2, p. 228-237, 1994. doi: 10.1006/jmra.1994.1209.

38 BENNER, P.; FASSBENDER, H. An implicitly restarted symplectic Lanczos method for the Hamiltonian eigenvalue problem. Linear Algebra and its Applications, v. 263, p. 75-111, 1997. doi: 10.1016/S0024-3795(96)00524-1.

39 MANDELSHTAM, V. A.; TAYLOR, N. D.; HU, H.; SMITH, M.; SHAKA, A. Highly resolved double absorption 2D NMR spectra from complex severely truncated 2D phase-modulated signals by filter-diagonalization-averaging method. Chemical Physics Letters, v. 305, n. 3-4, p. 209-216, 1999. doi: 10.1016/S0009-2614(99)00395-4.

$40 \mathrm{HU}, \mathrm{H} . ;$ filter diagonalization method. II. applications to 2D, 3D and 4D NMR experiments. Journal of Magnetic Resonance, v. 144, n. 2, p. 357-366, 2000. doi: 10.1006/jmre.2000.2066.

41 CHEN, J.; MANDELSHTAM, V. A.; SHAKA, A. Regularization of the two-dimensional filter diagonalization method: FDM2K. Journal of Magnetic Resonance, v. 146, n. 2, p. 363-368, 2000. doi: 10.1006/jmre.2000.2155.

42 KOGBETLIANTZ, E. G. Solution of linear equations by diagonalization of coefficients matrix. Quarterly of Applied Mathematics, v. 13, n. 2, p. 123-132, 1955.

43 HESTENES, M. R. Inversion of matrices by biorthogonalization and related results. Journal of the Society for Industrial and Applied Mathematics, v. 6, n. 1, p. 51-90, 1958.

44 FORSYTHE, G. E.; HENRICI, P. The cyclic Jacobi method for computing the principal values of a complex matrix. Transactions of the American Mathematical Society, v. 94, n. 1, p. 1 , 1960. doi: $10.2307 / 1993275$. 
45 GU, M.; EISENSTAT, S. C. A divide-and-conquer algorithm for the bidiagonal SVD. SIAM Journal on Matrix Analysis and Applications, v. 16, n. 1, p. 79-92, 1995. doi: $10.1137 /$ S0895479892242232.

46 GROSSER, B.; LANG, B. An O(n2) algorithm for the bidiagonal SVD. Linear Algebra and its Applications, v. 358, n. 1-3, p. 45-70, 2003. doi: 10.1016/S0024-3795(01)00398-6.

47 LI, S.; GU, M.; CHENG, L.; CHI, X.; SUN, M. An accelerated divide-and-conquer algorithm for the bidiagonal SVD problem. SIAM Journal on Matrix Analysis and Applications, v. 35, n. 3, p. 1038-1057, 2014. doi: 10.1137/130945995.

48 HOGBEN, L. Handbook of linear algebra. Boca Raton: CRC Press, 2016.

49 MOLER, C. B.; STEWART, G. W. An algorithm for generalized matrix eigenvalue problems. SIAM Journal on Numerical Analysis, v. 10, n. 2, p. 241-256, 1973. doi: $10.1137 / 0710024$.

50 WATKINS, D. S. The matrix eigenvalue problem. Philadelphia: Society for Industrial and Applied Mathematics, 2007.

51 HADLOCK, C. R. Field theory and its classical problems. Washington: The Mathematical Association of America, 1978. (The Carus mathematical monographs, 19).

52 GOLUB, G. H. Matrix computations. Baltimore: Johns Hopkins University Press, 2012.

53 MANDELSHTAM, V. A.; VAN, Q. N.; SHAKA, A. J. Obtaining proton chemical shifts and multiplets from several 1D NMR signals [19]. Journal of the American Chemical Society, v. 120, n. 46 , p. $12161-12162,1998$. doi: $10.1021 /$ ja9824977.

54 VANHAMME, L.; VAN DEN BOOGAART, A.; Van Huffel, S. Improved method for accurate and efficient quantification of MRS data with use of prior knowledge. Journal of Magnetic Resonance, v. 129, n. 1, p. 35-43, 1997. doi: 10.1006/jmre.1997.1244.

55 ALDENDERFER, M.; BLASHFIELD, R. Cluster analysis. California: Sage Publications, 1984.

56 JAIN, A. K.; DUBES, R. C. Algorithms for clustering data. Upper Saddle River: PrenticeHall, 1988.

57 EVERITT, B.; LANDAU, S.; LEESE, M.; STAHL, D. Cluster analysis. London: Wiley, 2011.

58 KAUFMAN, L.; ROUSSEEUW, P. Finding groups in data: an introduction to cluster analysis. New York: Wiley, 1990. 
59 XU, R.; Wunsch II, D. C. Clustering. New Jersey: John Wiley \& Sons, Inc., 2009.

60 GAMA, J.; FACELI, K.; LORENA, A.; DE CARVALHO, A. Inteligência artificial: uma abordagem de aprendizado de máquina. Rio de Janeiro: LTC, 2011.

$61 \mathrm{XU}, \mathrm{R}$.; WUNSCH, D. C. Clustering algorithms in biomedical research: a review. IEEE Reviews in Biomedical Engineering, v. 3, p. 120-154, 2010. doi: 10.1109/RBME.2010.2083647.

62 JAIN, A. K.; MURTY, M. N.; FLYNN, P. J. Data clustering: a review. ACM Computing Surveys, v. 31, n. 3, p. 264-323, 1999. doi: 10.1145/331499.331504.

$63 \mathrm{CHA}, \mathrm{S} .-\mathrm{H}$. Comprehensive survey on distance / similarity measures between probability density functions. International Journal of Mathematical Models and Methods in Applied Sciences, v. 1, n. 4, p. 300-307, 2007. doi: 10.1007/s00167-009-0884-z.

64 PEDREgOSA, F. et al. Scikit-learn: machine learning in Python. Journal of Machine Learning Research, v. 12, p. 2825-2830, 2011.

65 LLOYD, S. Least squares quantization in PCM. IEEE Transactions on Information Theory, v. 28, n. 2, p. 129-137, 1982. doi: 10.1109/TIT.1982.1056489.

66 FREY, B. J.; DUECK, D. Clustering by passing messages between data points. Science, v. 315, n. 5814, p. 972-976, 2007. doi: 10.1126/science.1136800.

$67 \mathrm{SHI}, \mathrm{J}$.; MALIK, J. Normalized cuts and image segmentation. IEEE Transactions on Pattern Analysis and Machine Intelligence, v. 22, n. 8, p. 888-905, 2000. doi: 10.1109/34.868688.

68 WARD JR., J. H. Hierarchical grouping to optimize an objective function. Journal of the American Statistical Association, v. 58, n. 301, p. 236-244, 1963. doi: 10.1080/01621459.1963.10500845.

69 ESTER, M.; KRIEGEL, H.-P.; SANDER, J.; XU, X. A density-based algorithm for discovering clusters in large spatial databases with noise. 1996. Disponível em: <https://www.aaai.org/Papers/KDD/1996/KDD96-037.pdf>. Acesso em: 17 out. 2019.

70 ANKERST, M.; BREUNIG, M. M.; KRIEGEL, H.-P.; SANDER, J. Optics: ordering points to identify the clustering structure. 1999. Disponível em: $<$ https://www.dbs.ifi.Imu.de/Publikationen/Papers/OPTICS.pdf >. Acesso em: 17 out. 2019.

71 ZHANG, T.; RAMAKRISHNAN, R.; LIVNY, M. Birch: an efficient data clustering method for very large databases. 1996. Disponível em: $<$ https://www2.cs.sfu.ca/CourseCentral/459/han/papers/zhang96.pdf>. Acesso em: 17 out. 2019. 
72 DEMPSTER, A. P.; LAIRD, N. M.; RUBIN, D. B. Maximum likelihood from incomplete data via the EM algorithm. Journal of the Royal Statistical Society Series B: methodological, v. 39, n. 1, p. 1-22, 1977. doi: 10.1111/j.2517-6161.1977.tb01600.x.

73 WIKIPEDIA. DBSCAN. 2019. Disponível em: $<$ https://en.wikipedia.org/wiki/DBSCAN>. Acesso em: 17 out. 2019.

74 SILVA, D. M. D. D.; VAZ, Y.; PAIVA, F. F. MRS data deconvolution through KBDM with multiple signal truncation and clustering: circumventing noise effects. In: WORLD CONGRESS ON MEDICAL PHYSICS AND BIOMEDICAL ENGINEERING, 2015, Toronto. Proceedings...Toronto: Springer, 2015. p. 1022-1025.

75 ROUSSEEUW, P. J. Silhouettes: a graphical aid to the interpretation and validation of cluster analysis. Journal of Computational and Applied Mathematics, v. 20, p. 53 - 65, 1987. doi: https://doi.org/10.1016/0377-0427(87)90125-7.

76 CAMPELLO, R. J.; MOULAVI, D.; SANDER, J. Density-based clustering based on hierarchical density estimates. In: ADVANCES IN KNOWLEDGE DISCOVERY AND DATA MINING, 17., 2013, Gold Coast, Australia. Proceedings...Heidelberg: Springer, 2013. p. 160-172. doi: 10.1007/978-3-642-37456-2_14.

77 CAMPELLO, R. J.; MOULAVI, D.; ZIMEK, A.; SANDER, J. Hierarchical density estimates for data clustering, visualization, and outlier detection. ACM Transactions on Knowledge Discovery from Data, v. 10, n. 1, p. 1-5, 2015. doi: 10.1145/2733381.

78 JARNÍK, V. Über ein Minimalproblem. Práce Moravské Přirodovědecké Společnosti. v. 6, p. 57-63, 1931.

79 PRIM, R. C. Shortest connection networks and some generalizations. Bell System Technical Journal, v. 36, n. 6, p. 1389-1401, 1957. doi: 10.1002/j.1538-7305.1957.tb01515.x.

80 GOWER, J. C.; ROSS, G. J. S. Minimum spanning trees and single linkage cluster analysis. Journal of the Royal Statistical Society Series C: applied statistics, v. 18, n. 1, p. 54-64, 1969.

81 CHAUDHURI, K.; DASGUPTA, S. Rates of convergence for the cluster tree. 2010. Disponível em: <http://papers.nips.cc/paper/4068-rates-of-convergence-for-the-cluster-tree.pdf>. Acesso em: 17 out. 2019.

82 CAMPELLO, R. J. G. B.; MOULAVI, D.; ZIMEK, A.; SANDER, J. A framework for semi-supervised and unsupervised optimal extraction of clusters from hierarchies. Data Mining and Knowledge Discovery, v. 27, n. 3, p. 344-371, 2013. doi: 10.1007/s10618-013-0311-4.

83 MCINNES, L.; HEALY, J.; ASTELS, S. hdbscan: hierarchical density based clustering. Journal of Open Source Software, v. 2, n. 11, 2017. doi: 10.21105/joss.00205. 
84 CAREY, C.; TANG, Y.; DE VAZELHES, W.; BELLET, A.; VAUQUIER, N. scikit-learncontrib, 2019. Disponível em: <https://github.com/scikit-learn-contrib>. Acesso em: 17 out. 2019.

85 BORUVKA, O. Über ein Minimalproblem. Práce Moravské Přirodovědecké Společnosti. v. 3, p. 37-58, 1926.

86 CONDA-FORGE. conda-forge community driven packaging for conda. 2019. Disponível em: <https://conda-forge.org/>. Acesso em: 17 out. 2019.

87 OLIPHANT, T. E. Python for scientific computing. Computing in Science \& Engineering, v. 9, n. 3, p. 10-20, 2007. doi: 10.1109/MCSE.2007.58.

88 VAN DER WALT, S.; COLBERT, S. C.; VAROQUAUX, G. The NumPy array: a structure for efficient numerical computation. Computing in Science Engineering, v. 13, n. 2, p. 22-30, 2011. doi: 10.1109/MCSE.2011.37.

89 JONES, E.; OLIPHANT, T.; PETERSON, P. SciPy: open source scientific tools for Python. 2019. Disponível em: <http://www.scipy.org>. Acesso em: 17 out. 2019.

90 MCKINNEY, W. Data structures for statistical computing in Python. In: PYTHON IN SCIENCE CONFERENCE, 9., 2010, Austin. Proceedings...Austin: SciPy, 2010. p. $51-56$.

91 NETLIB. LAPACK - linear algebra package. 2019. Disponível em: <http://www.netlib.org/lapack>. Acesso em: 17 out. 2019.

92 INTEL. Developer reference for Intel $\AA$ math kernel library - C. 2019. Disponível em: $<$ https://software.intel.com/en-us/mkl-developer-reference-c-lapack-routines $>$. Acesso em: 17 out. 2019.

93 RIVERBANK. Software. PyQt. what is PyQt?. 2019. Disponível em: $<$ https://riverbankcomputing.com/software/pyqt/intro/>. Acesso em: 17 out. 2019.

94 CAMPAGNOLA, L. PyQtGraph - scientific graphics and GUI library for Python. 2019. Disponível em: <http://www. pyqtgraph.org/>. Acesso em: 17 out. 2019.

95 ROWLAND, B. Suspect documentation! - suspect 0.3.9 documentation. 2019. Disponível em: <https://suspect.readthedocs.io/en/latest/>. Acesso em: 17 out. 2019.

96 KREKEL, H.; PFANNSCHMIDT, R.; OLIVEIRA, B. pytest: helps you write better programs - pytest documentation. 2019. Disponível em: <https://docs.pytest.org/en/latest/>. Acesso em: 17 out. 2019. 
97 NARESSI, A.; COUTURIER, C.; DEVOS, J.; JANSSEN, M.; MANGEAT, C.; DE BEER, R.; GRAVERON-DEMILLY, D. Java-based graphical user interface for the MRUI quantitation package. Magnetic Resonance Materials in Physics, Biology and Medicine, v. 12, n. 2-3, p. 141, 2001.

98 STEFAN, D.; DI CESARE, F.; ANDRASESCU, A.; POPA, E.; LAZARIEV, A.; VESCOVO, E.; STRBAK, O.; WILLIAMS, S.; STARCUK, Z.; CABANAS, M. Quantitation of magnetic resonance spectroscopy signals: the jMRUI software package. Measurement Science and Technology, v. 20, n. 10, p. 104035, 2009.

99 SCIPY. scipy.stats.normaltest - SciPy v1.3.1 reference guide. 2019. Disponível em: $<$ https://docs.scipy.org/doc/scipy/reference/generated/scipy.stats.normaltest.html >. Acesso em: 17 out. 2019. 